\title{
Integrated approaches for marine actinomycete biodiscovery
}

\author{
Larissa Buedenbender
}

B. Science (Honours)

Griffith University

Submitted in the fulfilment of the requirements of the degree of Doctor of Philosophy

\section{W/J Griffith}

Griffith School of Environment

Griffith Sciences

Griffith University, Australia 


\section{ABSTRACT}

This $\mathrm{PhD}$ project examined integrated approaches for marine actinomycete biodiscovery. A library of 120 actinomycete strains derived from the three Australian ascidians, Symplegma rubra, Aplidium solidium and Polyclinum vasculosum, was established in order to access new chemical diversity of underexplored marine ecological niches for natural product drug discovery. Specifically, the genera Streptomyces and Micromonospora were highly diverse and abundantly present, while fewer Nocardia and Rhodococcus and only one Streptosporangium representative were isolated. Only two isolates occurred in all three ascidians indicating speciesspecificity of actinomycetes in the ascidian. LC-MS/MS profiling of extracts obtained from the ascidians and their actinomycete associates revealed many overlapping ions between hosts and cultured isolates indicating that these compounds were likely to be synthesised by the microbial associates. Laboratory cultures of the actinomycetes displayed even more diverse metabolomes than those of their ascidian hosts; thus making ascidian-associated actinomycetes an excellent target for biodiscovery.

Reisolation of already known compounds is a major obstacle to natural product drug discovery; therefore, sophisticated dereplication approaches have to be employed in the early stages of discovery. To facilitate the dereplication, a new strain prioritisation approach using HSQC-TOCSY NMR spectra together with anti-plasmodial activity data was developed. This allowed for prioritisation of microbial strains that are more likely to produce diverse polyketide or peptide natural products. This approach was further validated through dereplication of monoand co-culture extracts of four different microbial strains. HSQC-TOCSY NMR profiles of the extracts clearly showed co-cultivation induced changes in the microbial metabolomes.

Streptomyces sp. (USC-16018) was selected based on the strain prioritisation approach and large-scale fermentations led to the isolation of a new ansamycin polyketide, herbimycin $\mathrm{G}$ with weak anti-plasmodial activity (77\% at $40 \mu \mathrm{M})$, as well as the known compounds elaiophylin, and the four diketiopiperazines, Cyclo-L-Pro-L-Leu, Cyclo-L-Pro-L-Phe, Cyclo-L-Pro-L-Val and Cyclo-L-Pro-L-Tyr. The crude NMR profile of the sediment-derived Streptomyces sp. (USC636) revealed the presence of indole-associated signals and three tryptophan containing diketopiperazines, naseseazine $\mathrm{A}-\mathrm{C}$, were isolated. The new compound naseseazine $\mathrm{C}$ exhibited moderate anti-plasmodial activity $\left(\mathrm{IC}_{50} 3.52 \mu \mathrm{M}\right)$.

Overall, this thesis highlights the immense chemical diversity of actinomycetes from marine sources, as well as the importance of sophisticated dereplication approaches and provides an alternative new solution for strain selection that has led to the discovery of new bioactive natural products with potential as new drug leads. 


\section{STATEMENT OF ORIGINALITY}

This work has not previously been submitted for a degree or diploma in any university. To the best of my knowledge and belief, the thesis contains no material previously published or written by another person except where due reference is made in the thesis itself.

(Signed)

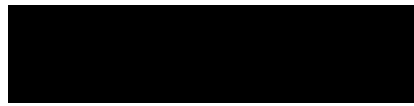

(Date) $\underline{01 / 09 / 2017}$

Larissa Buedenbender 


\section{TABLE OF CONTENT}

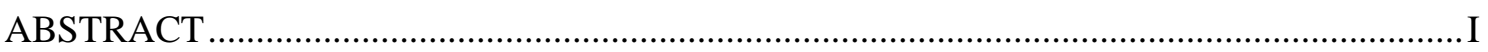

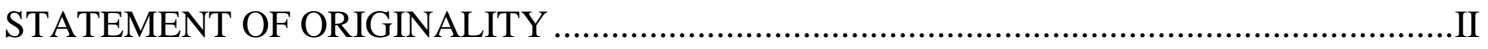

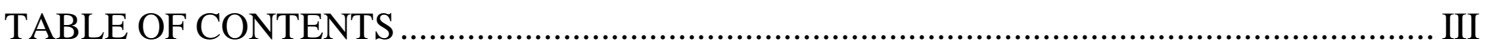

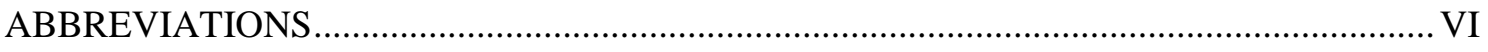

ALL PAPERS INCLUDED ARE CO-AUTHORED ...........................................................

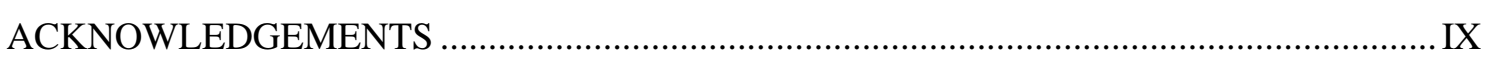

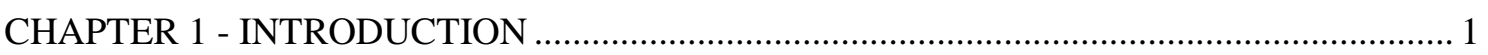

1.1. Microbial natural product in drug discovery ............................................................... 1

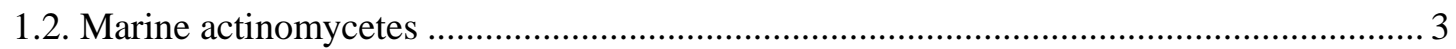

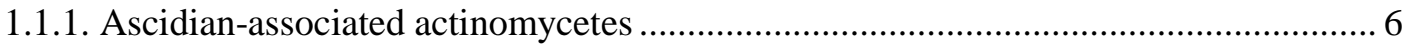

1.2. Potential and hurdles in marine microbial drug discovery ............................................... 8

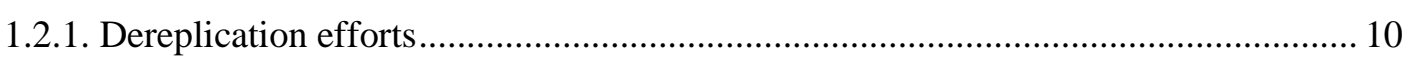

1.2.2. One strain many compounds (OSMAC) approach................................................ 13

1.2.3. Elicitation of the microbial metabolome: community cultures ................................. 13

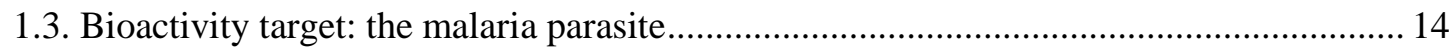

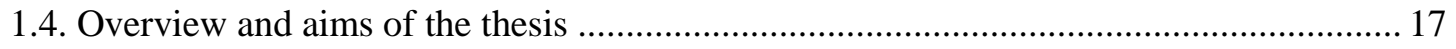

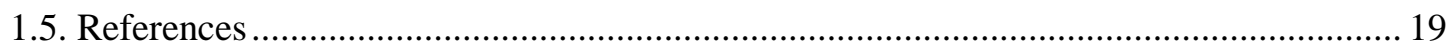

SECTION 1 Untapped ecological niche: Ascidian-associated actinomycetes........................... 24

CHAPTER 2 - Taxonomic and metabolite diversity of actinomycetes associated with three

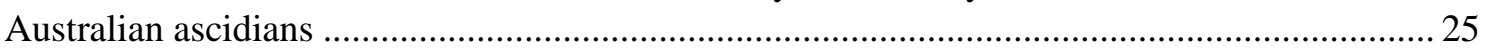

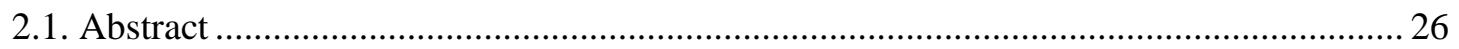

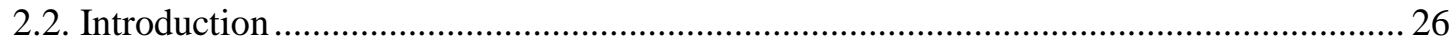

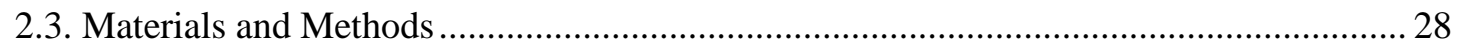

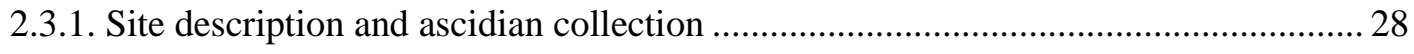

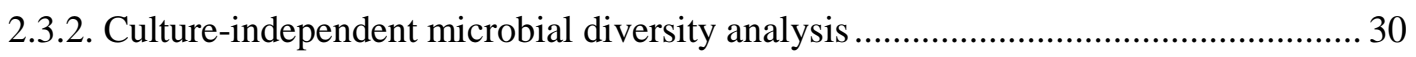

2.3.3. Culture-dependent actinomycete isolation and identification .................................. 30

2.3.4. Molecular identification and phylogenetic analysis of actinomycete isolates ........... 31

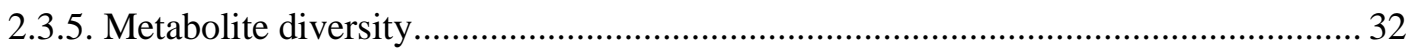

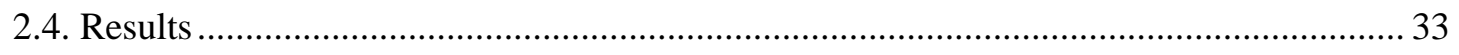

2.4.1. Microbial diversity associated with three Australian ascidians ............................... 33

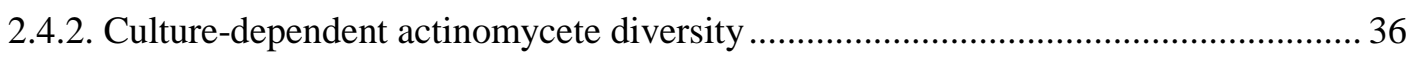

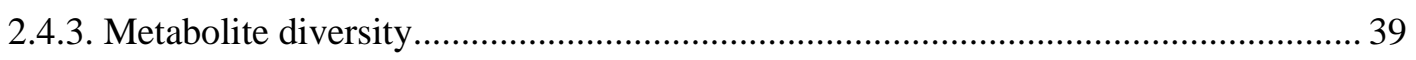

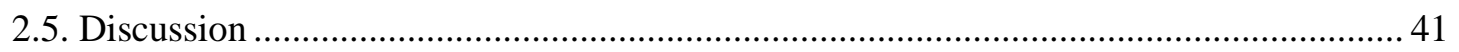

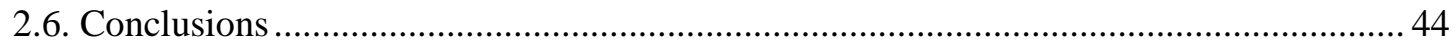

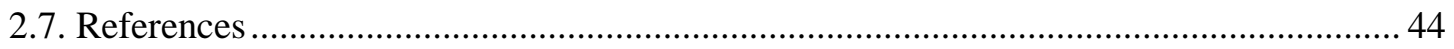


CHAPTER 3 - HSQC-TOCSY NMR profiling: using informative metabolomics to prioritise microbial strains for natural product drug discovery ................................................................ 50

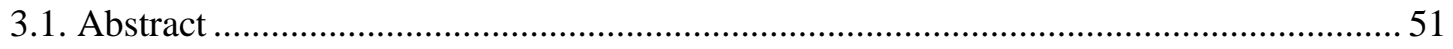

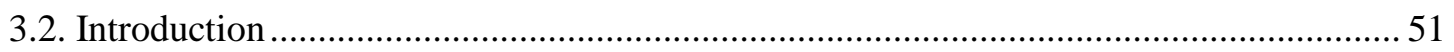

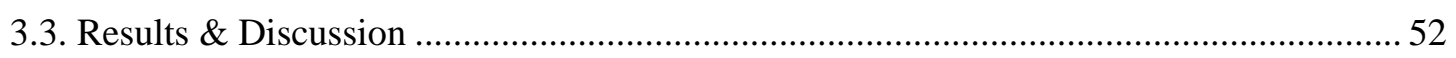

3.3.1. Determination of microbial culturing and chemical extraction protocol ...................52

3.3.2. Identification of regions and peaks of interest in 2D HSQC-TOCSY NMR spectra 54

3.3.3. Integrating metabolomics tools for microbial strain prioritisation from a library of

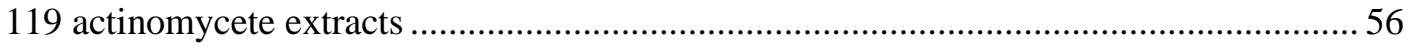

3.3.4. Discovery of bioactive natural products from selected strains................................... 61

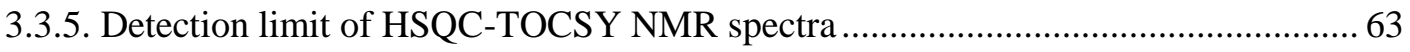

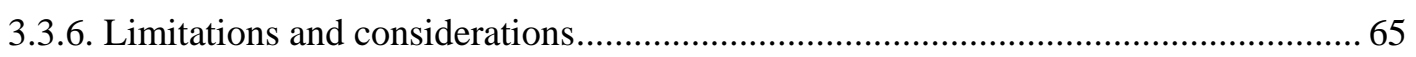

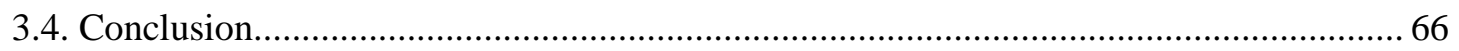

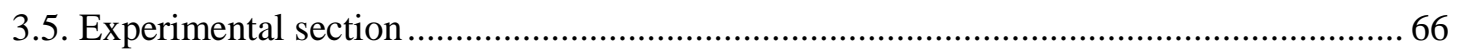

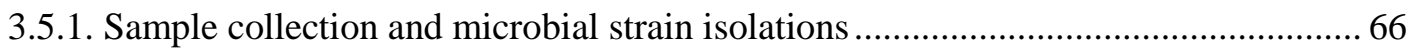

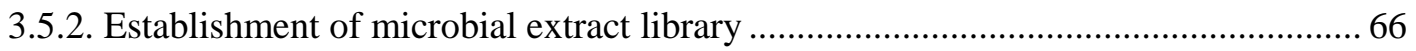

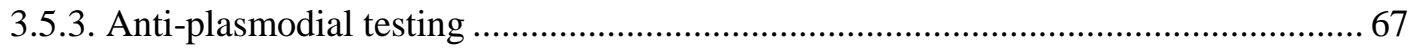

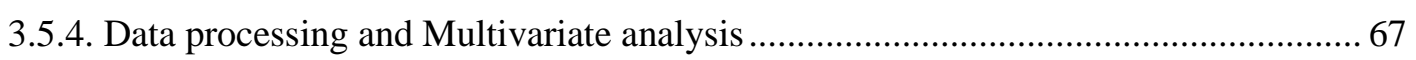

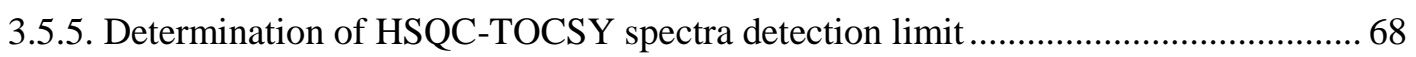

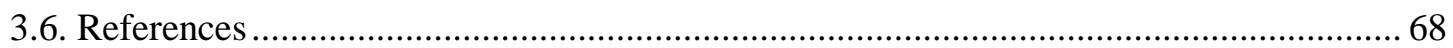

CHAPTER 4 - Detecting chemical diversity: HSQC-TOCSY NMR spectra show cocultivation-induced secondary metabolite production .............................................................. 70

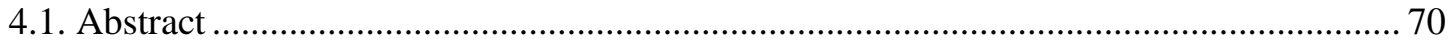

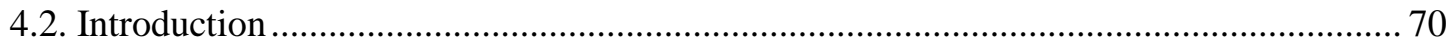

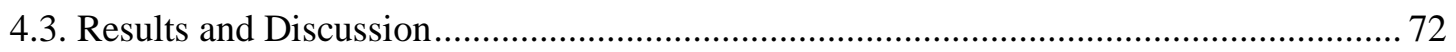

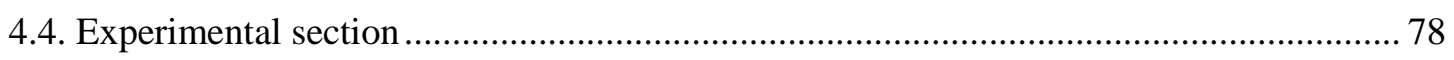

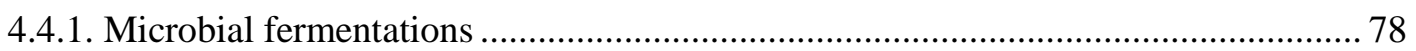

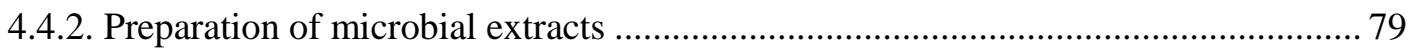

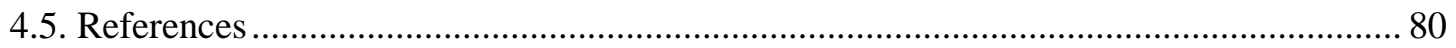

SECTION 3 Marine actinomycetes as a source of new anti-plasmodial compounds ................ 81

CHAPTER 5 - HSQC-TOCSY profiling directed discovery of anti-plasmodial polyketide compounds in marine Streptomyces species (USC-16018) ...................................................... 82

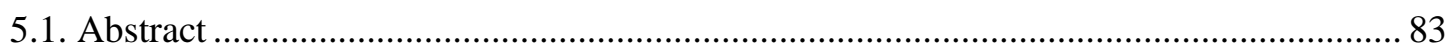

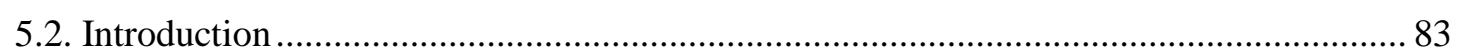

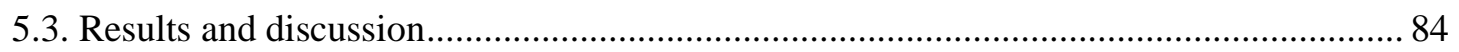

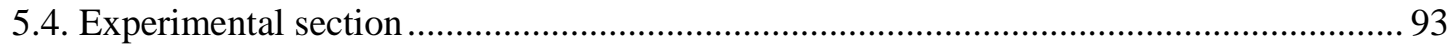

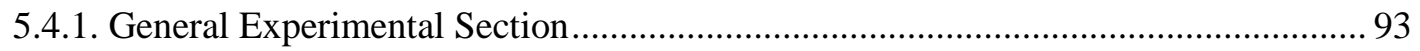




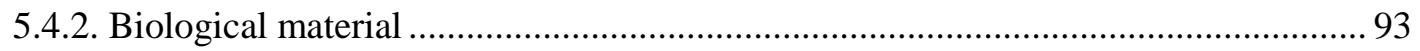

5.4.3. Fermentation, Extraction and Isolation ................................................................... 94

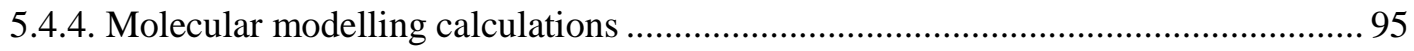

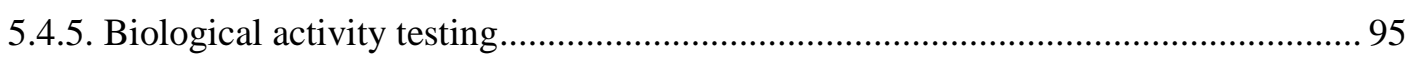

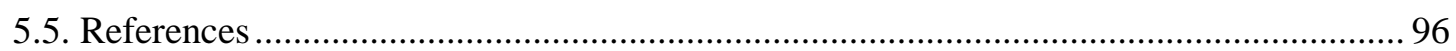

CHAPTER 6 - Naseseazine C, a new anti-plasmodial dimeric diketopiperazine from a marine

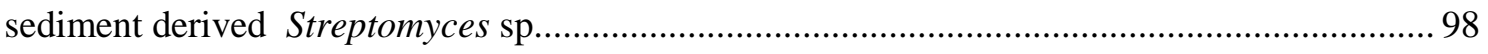

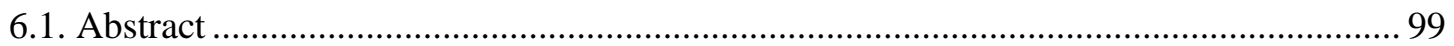

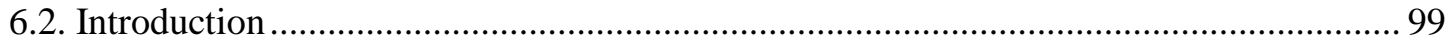

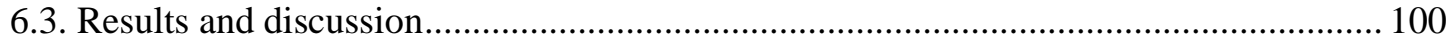

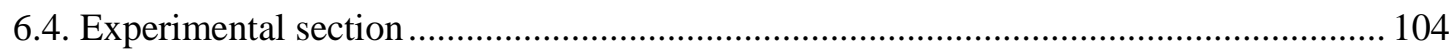

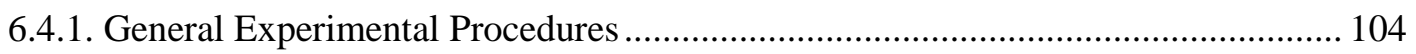

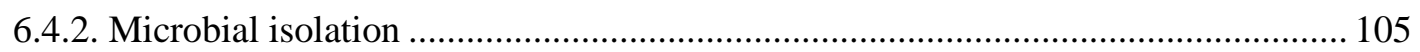

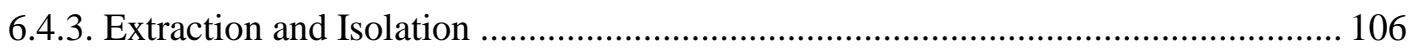

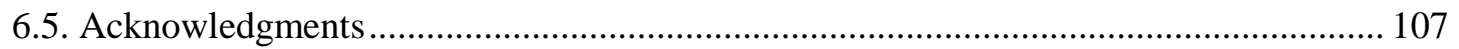

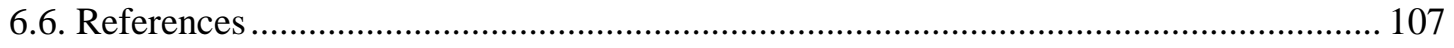

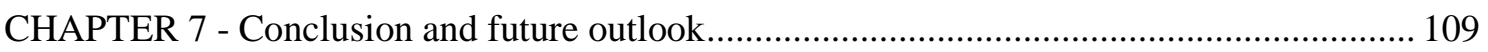

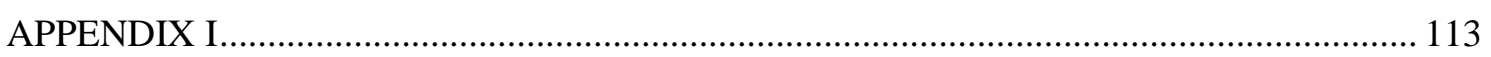

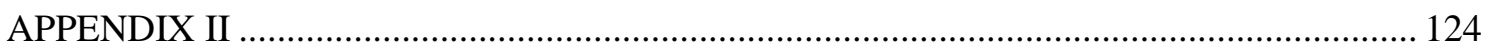

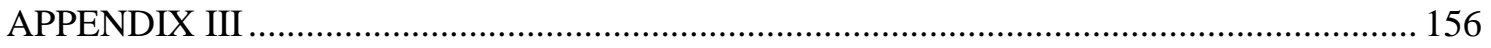

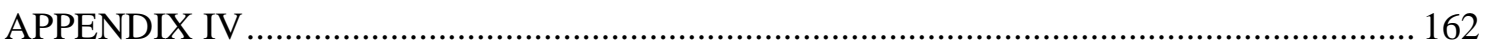

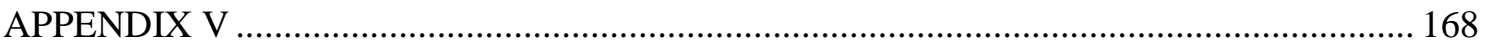




\section{ABBREVIATIONS}

\begin{tabular}{|c|c|}
\hline 16S rRNA & $16 \mathrm{~S}$ ribosomal RNA subunit \\
\hline ATCC & American Type Culture Collection \\
\hline BLAST & Basic Local Alignment Search Tool \\
\hline bp & Base pair \\
\hline $\operatorname{clog} P$ & Logarithm of its partition coefficient between n-octanol and water $\log \left(\mathrm{c}_{\mathrm{octanol}} / \mathrm{c}_{\mathrm{water}}\right)$ \\
\hline COSY & Correlation Spectroscopy \\
\hline $\mathrm{CS}$ & Corn starch media \\
\hline DMSO & Dimethyl sulfoxide \\
\hline DNA & Deoxyribonucleic acid \\
\hline ECD & Electronic circular dichroism \\
\hline ESI & Electrospray Ionization \\
\hline $\mathrm{EtOH}$ & Ethanol \\
\hline FDA & Food and Drug Administration \\
\hline HMBC & Heteronuclear Multiple Bond Correlation \\
\hline HRESIMS & High-Resolution Electrospray Ionisation Mass Spectrometry \\
\hline Hsp90 & Heat shock protein 90 \\
\hline HSQC & Heteronuclear Single Quantum Coherence/Correlation \\
\hline HTS & High Throughput Screening \\
\hline IC50 & Half maximal Inhibitory Concentration \\
\hline MA & Marine agar \\
\hline $\mathrm{MeOH}$ & Methanol \\
\hline MS & Mass Spectrometry \\
\hline NCBI & National Center for Biotechnology Information \\
\hline $\mathrm{nM}$ & Nano molar \\
\hline NMR & Nuclear Magnetic Resonance \\
\hline NSW & New South Wales \\
\hline OMA & Oatmeal agar \\
\hline OTU & Operational Taxonomic Unit \\
\hline PCA & Principal component analysis \\
\hline PCoA & Principal coordinate analysis \\
\hline PCR & Polymerase chain reaction \\
\hline PEP & Peptide \\
\hline PK & Polyketide \\
\hline PKS & Polyketide synthesase \\
\hline PLS-DA & Partial least squares discriminant analysis \\
\hline PoI & Peaks of Interest \\
\hline QIIME & Quantitative Insights Into Microbial Ecology \\
\hline QM & Queensland Museum \\
\hline RNA & Ribonucleic acid \\
\hline ROESY & Rotating frame nuclear Overhauser Effect spectroscopy \\
\hline RPM & Revolutions per Minute \\
\hline TDDFT & Time-dependent density functional theory \\
\hline TOCSY & Total Correlation Spectroscopy \\
\hline USC & University of Sunshine Coast \\
\hline UV & Ultraviolet \\
\hline
\end{tabular}




\section{ALL PAPERS INCLUDED ARE CO-AUTHORED}

\section{Acknowledgement of Papers included in this Thesis}

Section 9.1 of the Griffith University Code for the Responsible Conduct of Research ("Criteria for Authorship"), in accordance with Section 5 of the Australian Code for the Responsible Conduct of Research, states:

To be named as an author, a researcher must have made a substantial scholarly contribution to the creative or scholarly work that constitutes the research output, and be able to take public responsibility for at least that part of the work they contributed. Attribution of authorship depends to some extent on the discipline and publisher policies, but in all cases, authorship must be based on substantial contributions in a combination of one or more of:

- $\quad$ conception and design of the research project

- $\quad$ analysis and interpretation of research data

- drafting or making significant parts of the creative or scholarly work or critically revising it so as to contribute significantly to the final output.

Section 9.3 of the Griffith University Code ("Responsibilities of Researchers"), in accordance with Section 5 of the Australian Code, states:

Researchers are expected to:

- $\quad$ Offer authorship to all people, including research trainees, who meet the criteria for authorship listed above, but only those people.

- $\quad$ accept or decline offers of authorship promptly in writing.

- Include in the list of authors only those who have accepted authorship

- Appoint one author to be the executive author to record authorship and manage correspondence about the work with the publisher and other interested parties.

- Acknowledge all those who have contributed to the research, facilities or materials but who do not qualify as authors, such as research assistants, technical staff, and advisors on cultural or community knowledge. Obtain written consent to name individuals. 
Included in this thesis are papers in Chapters 2 and 6 which are co-authored with other researchers. My contribution to each co-authored paper is outlined at the front of the relevant chapter. The bibliographic details (if published or accepted for publication)/status (if prepared or submitted for publication) for these papers including all authors, are:

Chapter 2: Buedenbender, L., Carroll, A.R., Ekins, M. Kurtböke, D.I., Diversity and chemical potential of actinomycetes associated with three Australian ascidians, Diversity, 2017, submitted for publication

Chapter 6: Buedenbender, L., Grkovic, T., Duffy, S., Kurtböke, D.I., Avery, V.M., Carroll, A.R., Naseseazine C, a new anti-plasmodial dimeric diketopiperazine from a marine sediment derived Streptomyces sp., Tetrahedron Letters, 2016, 57, 5893-5895. doi: http://dx.doi.org/10.1016/j.tetlet.2016.11.071

Appropriate acknowledgements of those who contributed to the research but did not qualify as authors are included in each paper.

(Signed)

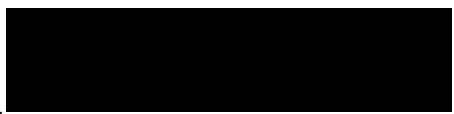

(Date) $\underline{01 / 09 / 2017}$

Larissa Buedenbender

(Countersigned)

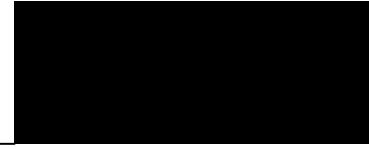

(Date) $\underline{31 / 08 / 2017}$

Principle supervisor: Anthony R. Carroll 


\section{Acknowledgments}

I am deeply grateful for receiving tuition fee and living allowances from Griffith University and the Australian Government during my candidature.

First of all, I would like to express my sincere gratitude to my principal supervisor Professor Anthony Carroll for the continuous support over the many years. Your expertise and great enthusiasm for natural product chemistry have been incredibly inspiring. Thank you for your patience and believing in me, guiding me, and giving me the freedom to develop new ideas.

My great appreciation goes to Dr Ipek Kurtböke for sharing her great knowledge and valuable skills in microbiology. Thank you for giving me the opportunity to work in your laboratory and for your kindness and encouragement. Your help was crucial to this $\mathrm{PhD}$ project. I would also like to thank Dr Tanja Grkovic for being such a great role model and mentor to me. I feel very fortunate that I have had the chance to work and learn from you. Thank you for giving me the confidence to believe in myself, and even from afar I could always count on your support.

Many people helped to make this research possible. On the technical side, I want to extend my gratitude to Dr Wendy Loa-Kum-Cheung for the NMR training and assistance, Dr. Maxim Sheludchenko and Ben Matthews for the help in the laboratory. Furthermore, I thank the brave Nathan Caromel and Johan Gustafson, who helped me sampling in rather cold conditions, and Professor Vicky M. Avery, Sandra Duffy and Dr Leonardo Lucantoni for screening my compounds and extracts. I would like to thank the staff and students at the University of Sunshine Coast that were incredibly welcoming and offered their assistance. I cannot thank Candice Brinkmann enough for patiently answering all my endless questions about microbiology and for being a good friend.

A very special thanks goes to my lab group, former and present; to Leesa Habener, Joshua Hayton, Fan Yang, Sarath (Dayani) Senadeera, Laurence Jennings, Luke Robertson, Guy Kleks, Shimony Mohanty, Marion Pactat, and Dr Casey Hall for creating such a friendly working environment. I especially want to thank Leesa for all the fruitful discussions about science and the world, and for all the motivation and help. I recognise all the efforts Josh and Luke put in for proof-reading my chapters and will forever be thankful for your support. 
Thank you to the administrative staff from the Griffith School of Environment and to Professor Catherine Pickering for convening the HDR program. I am overly grateful for the hospitality of Nick Yabsley and Ilana Kelly during my stay on the Sunshine Coast.

A big thank you goes to all my friends near and far, especially the 'climbing crew', who make my life enjoyable every day.

Finally, I thank my family, especially my parents and sister Bianca; your love and support even over a great distance gave me strength and courage in the good and the bad times. Equally, I thank my partner Carlos for his love, understanding and encouragement, and maintaining my happiness and sanity throughout this PhD. Thank you for always being there for me - I am so grateful to have you by my side. Here, I also need to include my extended Aussie family, Hoa Hoang, Hans Kainz, Tania Romero Brito and Jacqui Byrne, you know how to put me back on track and always manage to put a smile on my face. Every single encouraging word and good thought mattered. 


\section{CHAPTER 1 - INTRODUCTION}

\subsection{Microbial natural product in drug discovery}

Nature provides an enormous resource for biodiscovery that has been exploited by the pharmaceutical industry. Natural products (also known as secondary metabolites) are a highly diverse group of small molecules derived from living organisms. Unlike primary metabolites such as amino acids, carbohydrates and fatty acids that maintain function and growth of an organism; secondary metabolites are specific to their producer(s) but not essential for survival. ${ }^{1}$ The structural complexity of natural products is also closely linked to the ecological role of the producing organism and normally provides greater benefit in survival. ${ }^{2,3}$ Particularly, sessile or slow moving organisms rely more heavily on bioactive secondary metabolites, which act as defences, antimicrobials, allellochemicals, signalling molecules, UV protectants or feeding deterrents. ${ }^{4,5}$ The production of these compounds is energetically costly for the producing organism and thus they represent the most successful chemical structures, which form biologically friendly leads for drug development as they co-evolved with target sites in biological systems. ${ }^{1,6}$ Natural products are made by enzymes and thus present a greater number of chiral centres compared to synthetic compounds and their three-dimensional diversity aligns well with the majority of drugs on the market. ${ }^{2}$ Technological advancements and the advent of new tools such as nuclear magnetic resonance (NMR) and mass spectrometry (MS) have enhanced our understanding of natural products and aided the discovery of thousands of biologically active lead structures, drug candidates, and drugs to treat various diseases. ${ }^{7}$

Alexander Fleming's discovery of penicillin (1) from the fungus Penicillium notatum in 1928 and its subsequent development into an antibiotic in the 1940s was probably the most significant breakthrough in modern medicine, providing the foundation of drug discovery from microorganisms. ${ }^{8}$ The discovery of penicillin allowed for the treatment of bacterial infections, which until then were often fatal. Since then 120 natural products sourced from microorganisms, mainly from the bacterial order Actinomycetales (commonly termed actinomycetes), have found their way into the clinics as antimicrobials (i.e. rifamycin, 2), anticancer drugs (i.e. actinomycin, 3) and immunosuppressive agents (i.e. cyclosporin, 4) (Figure 1). 
<smiles>CC1(C)S[C@H]2C(NC(=O)Cc3ccccc3)C(=O)N2[C@H]1C(=O)O</smiles>

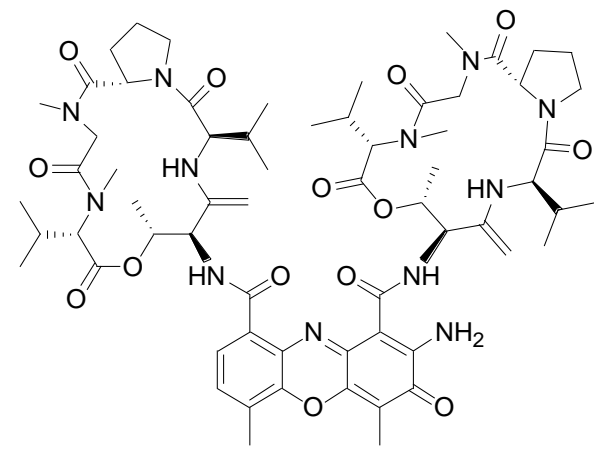

3

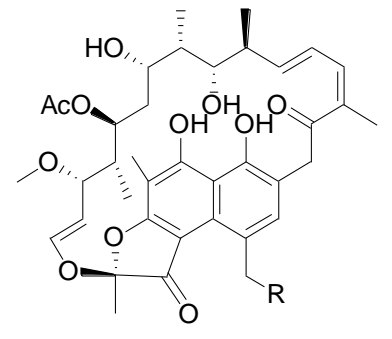

2a $\mathrm{R}=\mathrm{H}$

2b $\mathrm{R}=\mathrm{CCOOH}$

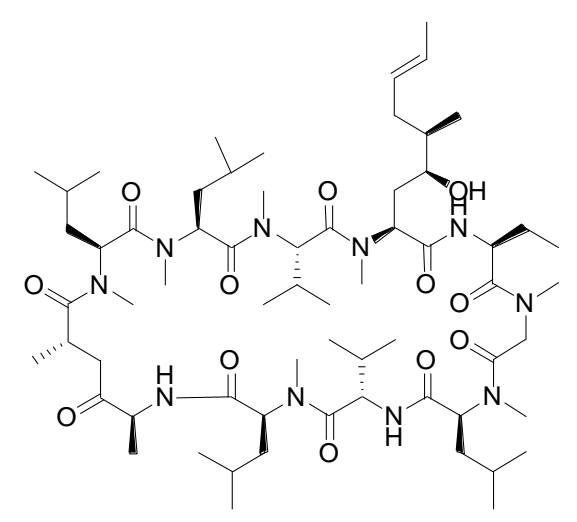

4

Figure 1. Important microbial drugs: Penicillin G (1), rifamycin (2), actinomycin (3) and cyclosporin (4).

Of all drugs newly approved between 1981 and 2014, $26 \%$ are natural products or natural product derived and $25 \%$ are natural product inspired synthetic drugs. ${ }^{9}$ Of the natural product derived drugs, $30 \%$ were isolated from microorganisms. This highlights the importance of natural products in drug discovery, particularly those from microbial sources. ${ }^{10}$ However, the time it takes from the discovery of a natural product until it becomes a marketed drug is very long and rediscovery of already known natural products has become more frequent. In the 1990's, high throughput screening (HTS) of combinatorial compound libraries became favoured by pharmaceutical companies as they are generally easier and cheaper to develop. ${ }^{11}$ Yet, the surge did not last long and the number of Food and Drug Administration (FDA) approved drugs decreased once pharmaceutical companies began to focus on purely synthetic drugs. ${ }^{12}$ Combinatorial compounds only occupy a very small area of chemical space, while natural products cover a much larger area of chemical space which aligns well with that of marketed drugs. ${ }^{2}$ Consequently, natural products and their derivatives remain of immense importance for new drug discovery and development. Nonetheless, to continue the successful exploitation of natural products for drug development, new approaches to increase chemical diversity were needed. 
One successful approach to enhance chemical diversity is to target organisms that have not previously been investigated. The high rediscovery of already known natural products from terrestrial bacteria has directed a focus to microorganisms from different ecological niches, particularly the marine environment, which covers $70 \%$ of the earth's surface and provides a vast and highly biodiverse resource for biodiscovery. ${ }^{13}$ The underlying hypothesis in marine biodiscovery is that taxonomic diversity correlates with chemical diversity and in recent years increased research has targeted species from the marine environment, which are taxonomically distinct to terrestrial organisms. ${ }^{13}$ It is believed that the physiochemical properties of the marine environment, including $\mathrm{pH}$, temperature, osmolarity, and pressure, as well as presence of uncommon halogenated functional groups result in natural products with enhanced bioactivities that have quite different properties compared to the terrestrial environment. ${ }^{13,14}$

A second way to minimise rediscovery is to implement sophisticated dereplication approaches early in the drug discovery efforts. An example of this is pre-screening of microbial strains and extracts with either molecular or spectrometric techniques to avoid repeatedly isolating the same microbial natural products. ${ }^{15}$ Another approach to maximise the great potential of bacteria for natural product drug discovery is to diversify culturing conditions. Microorganisms produce natural products in response to environmental stresses; therefore, using a range of different culturing media as well as chemical, physical and biological elicitors can trigger the biosynthesis of new metabolites. ${ }^{16}$ In the following, more detail on the approaches to diversify natural product biodiscovery from marine actinomycetes is provided.

\subsection{Marine actinomycetes}

Actinobacteria are Gram-positive and morphologically diverse bacteria. ${ }^{17}$ Currently, intraclass relatedness of the class actinobacteria reveals the presence of nine orders (http://www.bacterio.net/-classifphyla.html, accessed 14-08-2017). Accordingly, in this thesis "actinobacteria" will only refer to the "actinomycetes" covering the members of the order Actinomycetales. Actinomycetes are often filamentous and present diverse colony morphologies, which according to their function can be divided into areal hyphae, substrate mycelium, and in agar cultures often produce diffusible pigments (Figure 2). ${ }^{18,19}$ They inhabit a wide range of habitats from arid deserts to deep ocean sediments and are responsible for the production of many antibiotics, anticancer agents and immunosuppressive compounds. ${ }^{20,21}$ They are known to have symbiotic associations with plants, insects, and marine invertebrates. ${ }^{22-25}$ Actinomycetes, among other natural product producing bacteria, are the most prolific suppliers of bioactive compounds to the pharmaceutical industry. ${ }^{26}$ 


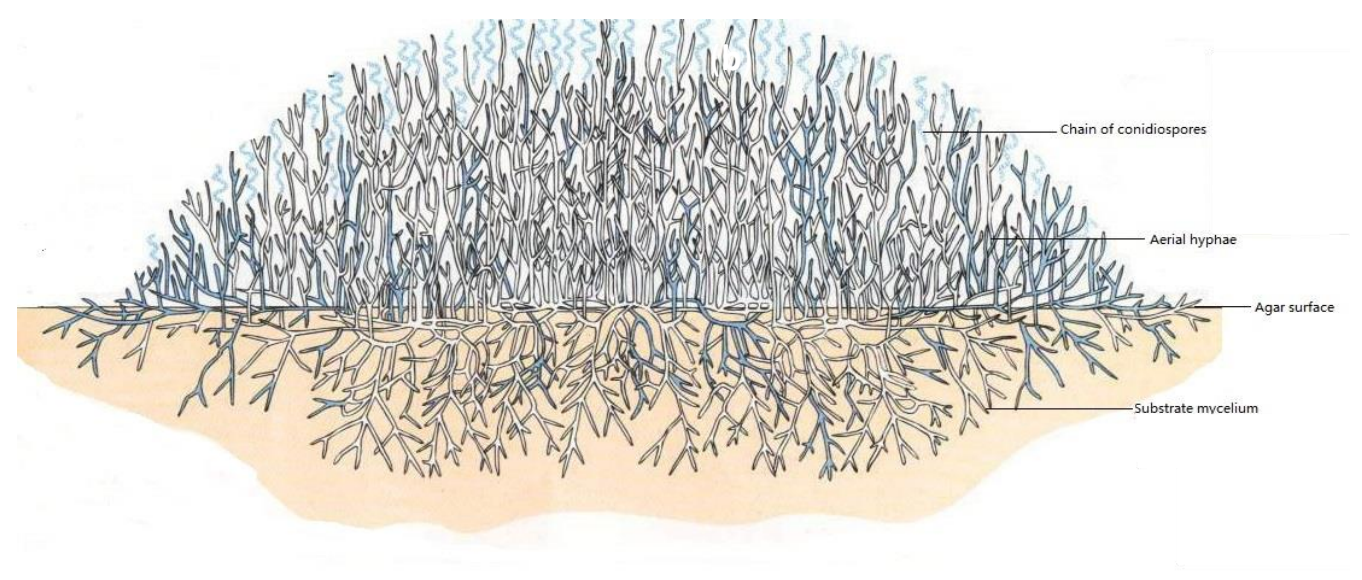

Figure 2. Common mycelial-forming actinomycete morphology on agar (colony cross section showing areal hyphae and substrate mycelium). ${ }^{19}$

Studies of marine actinomycetes date back to the 1940s, when the pioneers of marine microbiology, ZoBell and Rosenfeld, recognised the antimicrobial potential of marine microorganisms. ${ }^{27}$ However, only in the last two decades, extensive research in marine microbiology and chemistry has led to the isolation of many marine actinomycete species and the appreciation of their biosynthetic potential for drug discovery. The species described belong to the genera Dietzia, Streptomyces, Salinibacterium, Aeromicrobium, Williamsia, Verrucosispora Kocurea, and Polaribacter, as well as Salinispora, Demequina, Salinibacterium, Sciscionella, Serinococcus and Micromonospora. So far, only a small fraction of actinomycetes can be cultivated from the marine environment. True marine actinomycetes are generally difficult to culture in the laboratory, due to their special growth requirements. ${ }^{28,29}$ However, metagenomic data has revealed 10400 rRNA gene sequences of actinomycetes that are widely distributed throughout the marine environment; 16S rRNA genes have been isolated from the upper water column, different geological sediments as well as marine sponges, ascidians, and seaweeds. ${ }^{30}$ Development of new cultivation techniques allowed isolation of marine actinomycetes, which have produced novel and structurally diverse chemistry. ${ }^{31,32}$ Examples of bioactive marine actinomycete metabolites are: salinipyrones (anti-tumour, 5), proximicines (anti-tumour, antibiotic, 6), daryamides (anti-tumour, 7) or violaceins (antiprotozoal, 8) (Figure 3). ${ }^{33-36}$ The most promising discovery was salinosporamide A (9) produced by two rare Salinospora species. The compound is biosynthesized by an unusual hybrid PKS/NRPS pathway and features a bicyclic ring structure with a chloroethyl group and is functionalised with a cyclohexene. ${ }^{37}$ Extracts showed $\mathrm{IC}_{50}$ values of less than $2 \mathrm{ng} / \mathrm{mL}$ against human colon carcinoma HCT-116 cells and was found to be a highly selective tumour cell growth inhibitor. ${ }^{3853}$ Salinosporamide A, under the name Marizomib®, is currently undergoing clinical trial phase I in relapsed refractory multiple myeloma. ${ }^{39,40}$ 


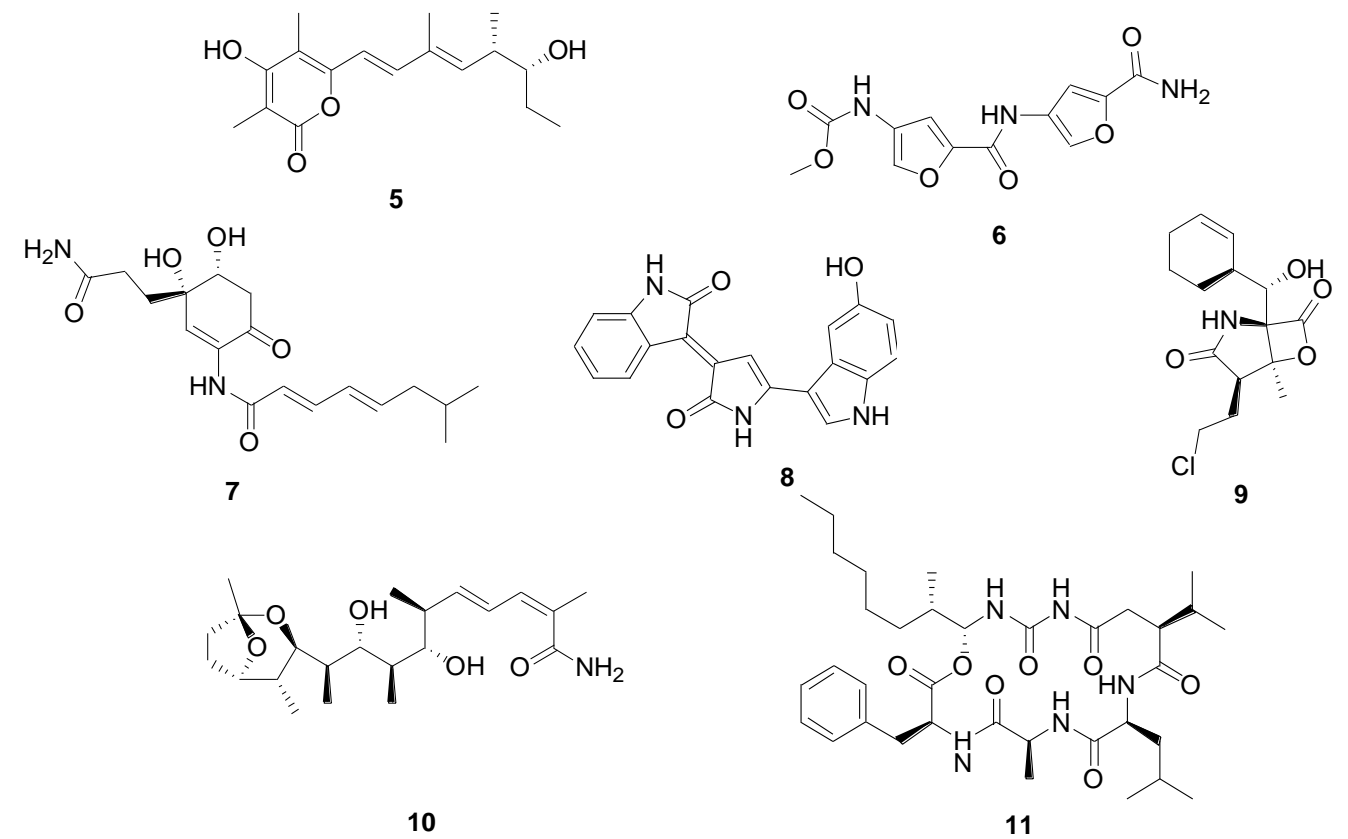

Figure 3. Examples of bioactive marine actinomycete metabolites: salinipyrone (5), proximycin A (6), daryamide A (7), violacein (8), salinosporamide A (9), saliniketal (10) and arenamide $\mathbf{A}(\mathbf{1 1})$.

Marine actinomycetes, like their terrestrial counterparts, have an extraordinary large genome, which points towards a great potential to biosynthesize secondary metabolites with biological activity. ${ }^{41}$ The first full genome sequenced from an obligate marine actinomycete species was that of Salinispora tropica strain CNB-440, one of the producers of salinosporaminde $\mathrm{A}$. The genome is composed of an average $\mathrm{G}+\mathrm{C}$ content of $69.5 \%$ and 17 secondary metabolic biosynthesis gene clusters, thus devoting approximately $10 \%$ of the genome to the production of natural products. ${ }^{42}$ Genome sequencing of the marine actinomycete Salinispora arenicola revealed 39 biosynthetic loci that produce secondary metabolites, ${ }^{43}$ including saliniketals (10) and arenamides (11). ${ }^{4445}$ However, it has become apparent that the majority of biosynthetic genes are silent under normal laboratory conditions or only low concentrations of natural products are produced. ${ }^{46}$ Genome sequences of Streptomyces coelicolor and Streptomyces avermitilis, two extensively studied species, also revealed interesting biosynthetic gene clusters with the potential to produce new natural products that are not yet expressed under the laboratory conditions. Thus, it seems necessary for marine actinomycete drug discovery that new culturing techniques and genome-based natural product mining develop in parallel. The culture-dependent approach is still very important in order to study the expressed bacterial metabolome, and if successful allows rapid assessment of structure, novelty and bioactivity. ${ }^{47}$ In contrast, the culture-independent approach will reveal the full biosynthetic potential of marine actinomycetes. 


\subsubsection{Ascidian-associated actinomycetes}

Ascidians are sessile, filter-feeding marine invertebrates known to produce highly potent bioactive natural products. ${ }^{48}$ These invertebrates are associated with a breadth of associated microorganisms and it has become evident that many of these bioactive metabolites are produced by an associated symbiont. ${ }^{49}$ Didemnin B and ecteinascidin 743 , two potent antitumour agents originally isolated from ascidians, have been proven to be of bacterial origin and similar evidence was found for about another 80 metabolites. ${ }^{50}$ The associations between microorganisms and ascidians have been described as species-specific and can be of obligate or facultative nature..$^{51,52}$

Actinomycetes are commonly detected by molecular sequencing techniques in many ascidians. ${ }^{53-56}$ Most studies have been unable to conclusively confirm any functional role of ascidian-associated actinomycetes. In an ascidian from the Microcosmus genus, actinomycetes were in particular associated with the digestive tract and it was therefore suspected that they were ingested from the water column through feeding. ${ }^{57}$ Actinomycetes play an important role in the digestion of recalcitrant organic matter in terrestrial invertebrates. Consequently, it could be possible that actinomycetes have a digestive functional role to play in ascidians that also feed on plankton containing high amounts of silica.

Furthermore, numerous bioactive compounds isolated from ascidians have remarkable similarities to actinomycete-derived metabolites (Figure 4). For instance, the ascidian antitumor compound, namenamicin (12), has striking structural similarity to calicheamicins (13) and esperamicins (14) both synthesised by actinomycetes. ${ }^{58-60}$ Several staurosporine derivatives (e.g. 15), a structure class known to be synthesised by terrestrial and marine actinomycetes, have repeatedly been isolated from Eudistoma ascidians, ${ }^{61}$ pointing towards a functional role of staurosporine production by an associated actinomycetes strain. ${ }^{62-64}$ Cultures of ascidianassociated actinomycetes have produced unique and novel chemistry under laboratory conditions; however, most studies were again inconclusive in revealing their functional roles in the ascidian-actinomycete association. In the hunt for the true producers of namenamicin, $\mathrm{He}$ and co-workers isolated a halophilic Micromonspora species, which produced lomaiviticins A (16) and B, two novel dimeric diazobenzofluorene glycosides that exhibit potent antitumor properties. ${ }^{63}$ A Streptomyces sp. strain isolated from Aplidium lenticulum at Heron Island, Australia, resulted in the discovery of the aromatic spiroketal polyketide griseorhodin A $(\mathbf{1 7})^{65}$ and Streptomyces sp. YM14-060 from an unidentified ascidian from Palau produced the new cytotoxic antibiotics, piericidins C7 and C8 (18). ${ }^{66}$ Another ascidian derived Micromonospora sp. yielded a new antimicrobial alkaloid, diazepinomicin (19) ${ }^{67}$; and peptidolipins B (20) -F with antibacterial properties were isolated from an ascidian-derived Nocardia species. ${ }^{68}$ 
Recently, Zhang and co-workers isolated micromonohalimanes A and B (21a/b) from a Micromonospora sp. associated with the ascidian, Symplegma brakenhielmi; this is the first account of such halimane-type diterpenoids from Micromonospora. ${ }^{69}$
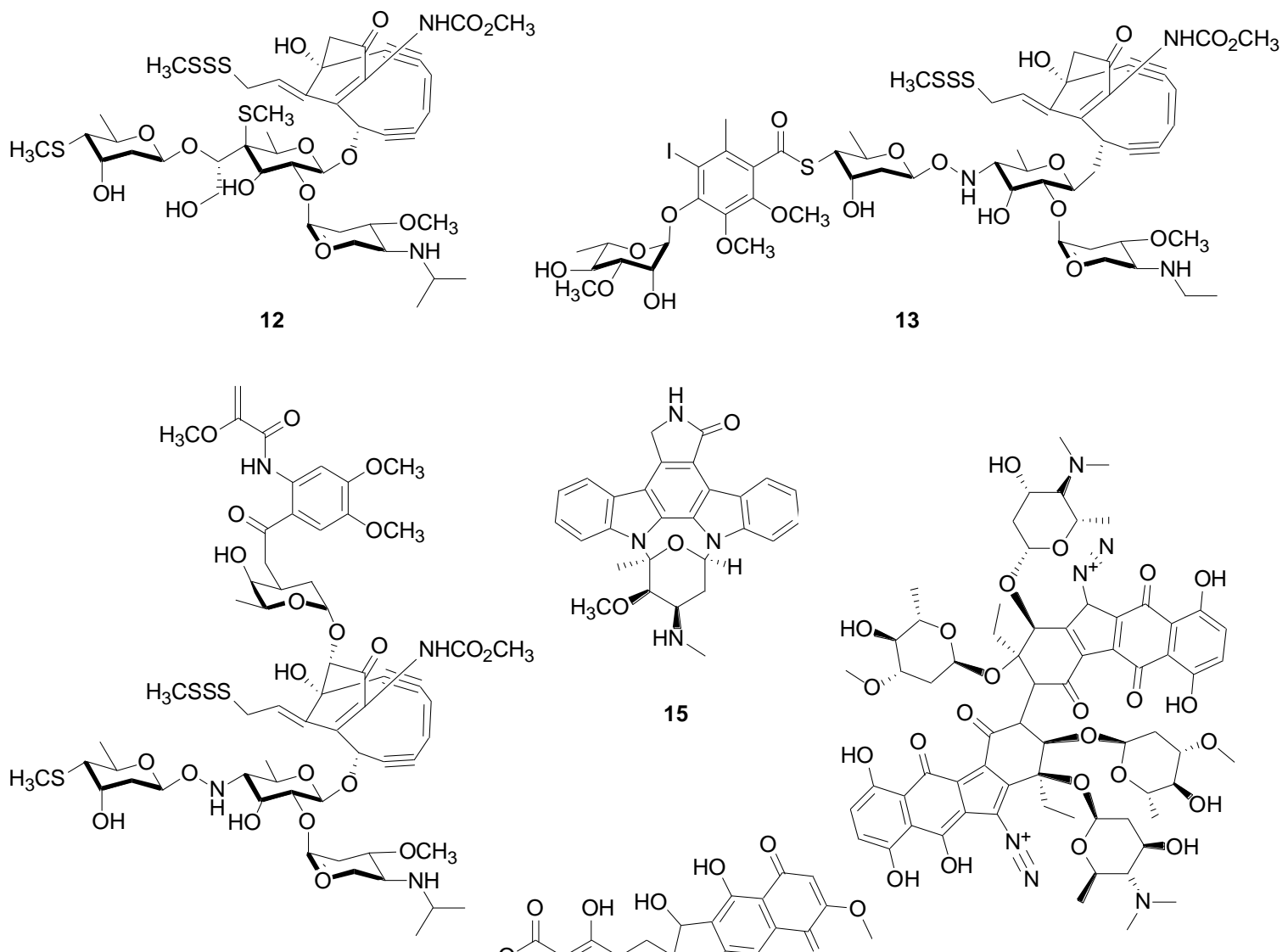

15

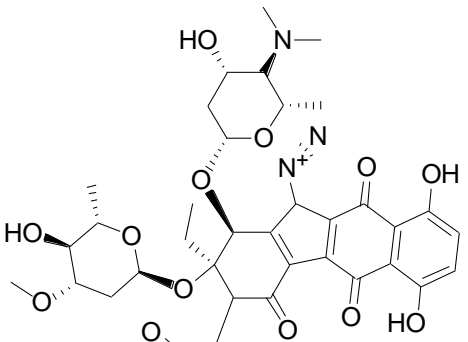

14
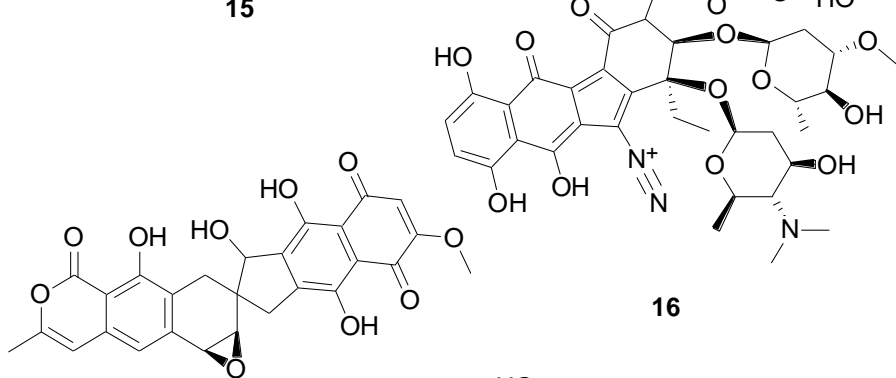

16

17
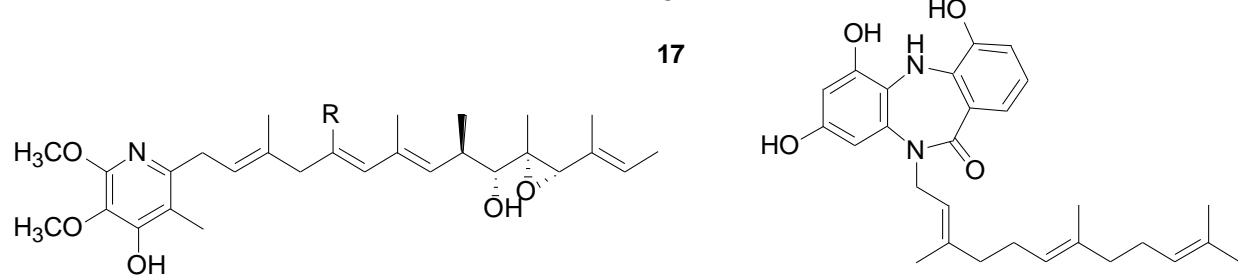

18a $\mathrm{R}=\mathrm{H}$

$18 b \mathrm{R}=\mathrm{CH}_{3}$

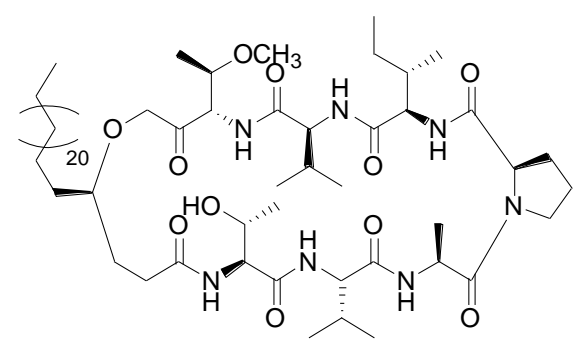

20

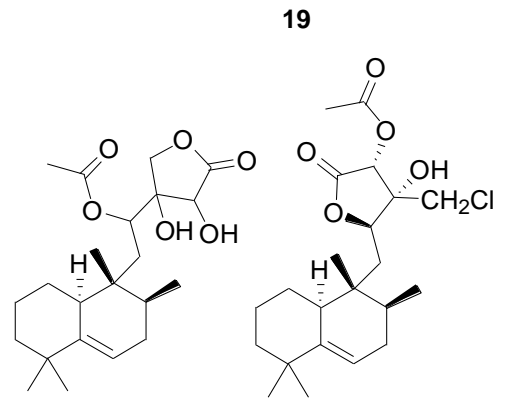

21a

21b

Figure 4. Diverse secondary metabolites derived or potentially derived from ascidianassociated actinomycetes 
Ascidian-associated actinomycetes are still an untapped source for natural product drug discovery, yet the few natural products that have been reported from ascidian-associated actinomycetes to date display an astonishing range of structural complexity, thus highlighting their potential for new drug discovery. The question whether actinomycetes play a functional role in the ascidian secondary metabolism is yet to be resolved. Much evidence points towards them playing a part in the metabolism, but more extensive studies, with newly developed culturing conditions that mimic ascidian-actinomycete associations more closely are needed to shed more light onto this mystery.

\subsection{Potential and hurdles in marine (microbial) drug discovery}

The re-supply of bioactive compounds derived from natural sources poses a major challenge, as natural populations of marine invertebrates are too small and often only minute amounts of the natural products are produced by the organisms. ${ }^{70}$ Nonetheless, natural product drug discovery has come a long way and it is now an interdisciplinary field which combines traditional natural products chemistry, synthetic chemistry, microbial- and molecular biology, metabolomics and toxicology. The union of these disciplines has resulted in a number of success stories.

One of the most significant true marine drugs is the ascidian-derived anti-tumour agent trabectedin (22), marketed as Yondelis ${ }^{\circledR}$. This compound was the first drug to be directly sourced from the marine environment. However' the producer Ecteinascidia turbinata only yielded $0.0001 \%$ of this compound. ${ }^{71}$ Five grams of the natural product were needed for clinical trials, and to produce this amount of compound, an unsustainably large quantity of tunicate biomass ( 5 tonnes) was required. ${ }^{72}$ Such extensive harvesting of the marine invertebrates can be restricted due to the general shortage of the marine organisms and can have adverse effects on the environment. ${ }^{70}$ In the most extreme case, extinction of the target species could result. ${ }^{73}$ Aquaculture and mariculture was then implemented to retrieve more biomass of that ascidian Ecteinascidia turbinata. ${ }^{71}$ However, yields of in-sea culturing are affected by environmental factors and often low, furthermore, diseases can spread easily; therefore, the pharmaceutical company PharmaMar needed to develop a new approach for full-scale production of the compound. ${ }^{74}$ The structure of trabectedin was inherently similar to the base structures of safracins (23) and saframycines (24) both derived from terrestrial bacteria, indicating that 22 was of microbial origin (Figure 5). ${ }^{75,76}$ PharmaMar established a semisynthetic process starting with the bacterial metabolite safracin $\mathrm{B}^{71}$ Total synthesis was also finally described, but the 
semisynthetic approach provided higher yields as well as additional related compounds. ${ }^{71,77}$ In 2007, 38 years after the original discovery of the compound, Yondelis ${ }^{\circledR}$ was approved as treatment for soft tissue sarcoma.

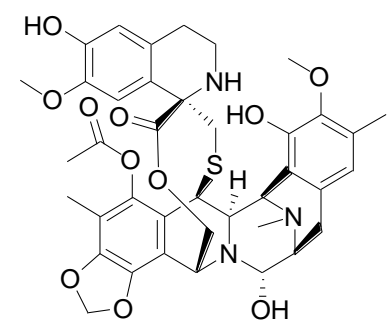

22

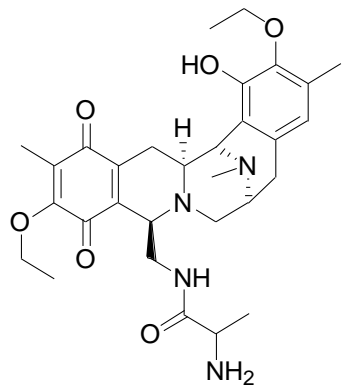

23

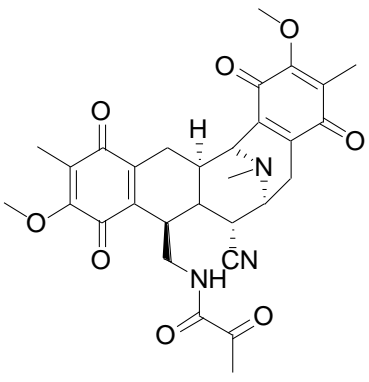

24

Figure 5. Trabectedin (22) and related safracin A (23) and saframycine A (24)

Equally impressive was the story of how eribulin became the marketed drug Halaven ${ }^{\circledR}$ for metastatic breast cancer. Halichondrin B was originally isolated from a marine sponge and exhibited very potent activity against murine leukaemia cells. Again, the supply of sufficient material for clinical development was the limiting factor. ${ }^{78}$ Total synthesis was achieved by Aicher and co-workers ${ }^{79}$, which was the most complex synthesis ever achieved. Subsequent research identified the active pharmacophore, which led to the synthesis of a simplified macrocyclic ketone analogue named eribulin mesylate with the same level of activity. ${ }^{72,80}$ This success story was the work of many international collaborators from industry, academia, and government, ultimately resulting in the approval of Halaven ${ }^{\circledR}$ in $2010 .^{78}$

As it becomes more evident that microorganisms are synthesising many natural products, cultivation of those microorganisms and heterologous gene expression techniques, where genes are expressed in a host organism, hold valuable alternatives to chemical synthesis or extraction from marine invertebrate sources. ${ }^{73}$ For example, the cytotoxic patellamides A (25) and C (26) (Figure 6), originally produced by the cyanobacterium Prochloron didemni, could be produced through heterologous gene expression in E. coli. ${ }^{51}$ While this approach seems very promising, at present it still has its constraints as not all genes will be expressed in all hosts, and it is likely that the target genes need specific chemical or environmental cues in order to be expressed. ${ }^{81}$ In conclusion, the supply problem is still the biggest hurdle to drug discovery from marine natural products, but examples like Yondelis ${ }^{\circledR}$ and Halaven ${ }^{\circledR}$ bring great hope to the natural product research community. Natural products possess many valuable and unique features and there is a continuous interest in the discovery of new organisms, especially from unusual ecological niches. ${ }^{82}$ New analytics and biotechnologies will further impel the field. 


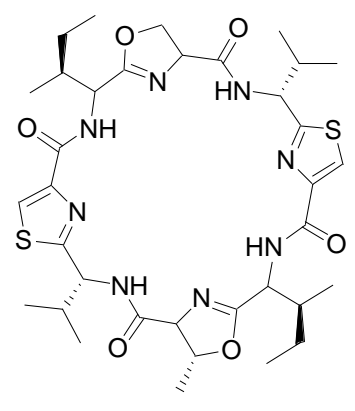

25

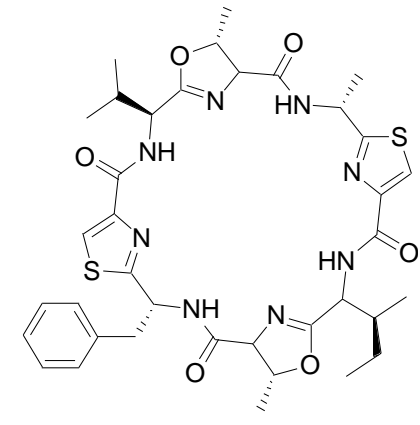

26

Figure 6. Patellamides A (25) and C (26) from ascidian-associated cyanobacteria

\subsubsection{Dereplication efforts}

Natural products are undoubtedly an important source of new drugs $;{ }^{9}$ however, biological screening, large-scale fermentation, isolation and structure elucidation are very resource- and time-intensive. Frequent rediscovery of already known natural products in the past decades has resulted in a decline in the use of natural products as sources for pharmaceuticals. Therefore, new analytical tools were required. In order to minimise rediscovery, efficient dereplication protocols have to be implemented early in natural product isolation efforts. Dereplication is the identification and elimination of known metabolites within samples that are targeted for new natural product discovery. ${ }^{83}$ This is particularly important for microbial natural products, where microbial strains cannot easily be distinguished through morphological features and it is not guaranteed that strains that optically appear different also produce different secondary metabolites. Conversely, strains that look the same might be able to produce totally distinct metabolomes. Dereplication can either be performed at the level of the microorganism or the chemical extracts.

\subsubsection{Taxonomic dereplication}

Molecular techniques currently allow dereplication at the genetic level. For decades, cluster analysis has been utilised to identify and dereplicate bacterial species. 16S rRNA sequencing is a widely accepted technique to identify environmental bacteria by using a common gene that possesses the universal primer of all bacteria that is distinct to the small subunit rRNA of eukaryotic organisms. ${ }^{84}$ Different microbial species have varying regions within the 16S rRNA sequence that, when matched up against $16 \mathrm{~S}$ gene databases, can be utilized for identification. Even microorganisms that cannot be identified to the species level will at least be placed in a group of related organisms. ${ }^{84}$ The American National Centre for Biotechnology Information (NCBI) offers an extensive open source database of nucleotide sequences and offers a basic 
local alignment search tool (BLAST) that matches input sequences against all deposited sequences. ${ }^{85}$ However, the ability to biosynthesise certain metabolites can vary even between different strains of the same taxonomic species ${ }^{86}$ therefore, culture dependent methods that prescreen microbial crude extracts are more commonly utilised by natural product chemists to evaluate the true potential of each bioactive strain.

\subsubsection{Chemical dereplication}

Traditionally, sample selection was based on biological pre-screening of the crude extract and subsequent bioassay-guided fractionation was used to identify the bioactive metabolites in the sample. ${ }^{87}$ This method is challenged by the repeated isolation of known natural products as it does not provide any chemical information about the active compounds in the extract. Recently, analytical spectroscopic and spectrometric data screening of the entire metabolome of an organism has become more popular in order to dereplicate samples, and more dereplication databases have emerged. ${ }^{15,88}$ Amongst others these include the Dictionary of Natural Products, AntiBase, MarinLit, GNPS and DEREP-NP. ${ }^{88-91}$

The two most common metabolomics profiling tools are LC-MS/MS and NMR profiling. ${ }^{92}$ The advantages of mass spectrometry are the high resolution and sensitivity, as well as the possibility to use MS/MS fragmentation. However, the technique is highly dependent on ionisation of compounds, is difficult to quantify and replicate between different instruments and thus not universally applicable. ${ }^{92}$ NMR techniques, on the other hand, have much lower resolution and sensitivity compared to MS, but NMR is non-selective, non-destructive, and quantitative, it requires minimal sample handling and undeniably its greatest advantage is that it provides structural information about the constituents of the extracts. ${ }^{92}$

Metabolomics was originally aimed at studying the total metabolomic processes within organisms and mostly applied in the human health sector, but also plant research has advanced the field of metabolomics. ${ }^{93}$ More recently its application has become appreciated in natural products drug discovery, particularly for the purpose of dereplication and mode of action studies. ${ }^{93}$ Chemometric-metabolomics profiling approaches provide an overview of the expressed metabolites in extracts, reduce redundancy and can be used for large datasets without comprehensive fractionation. In chemometric-metabolomics approaches, the chemical profiles are subsequently analysed using multivariate statistics, such as Principle Component Analysis (PCA) or Partial Least Squares Discriminant Analysis (PLS-DA). PCA is an unsupervised method that reduces multivariate data to a few principle components, while PLS-DA is a supervised technique that determines the variation within a dataset based on classification labels. ${ }^{94}$ These techniques produce a scores plot that allows visualisation of the spectral 
variations of the samples. Samples containing the same or similar chemical profiles will cluster together while differing profiles will cluster away from each other.

Hou and co-workers ${ }^{95}$ analysed a microbial collection of 47 samples using LC-MS and utilised PCA to prioritise chemically unique microbial strains for natural product discovery. They noted that one of the limiting factor to this analysis is that it is not readily applicable for large datasets and rather proposed a successive analysis of a smaller number of microbial isolates $\left(20-50\right.$ at a time). ${ }^{95}$ Even in their analysis of 47 isolate samples, over 25000 bucket variables derived from these samples and 74 principle components were needed to explain $98 \%$ of the variation in the dataset, indicating that the first two principle components most likely only accounted for a very small percentage of the variation in the data. Nonetheless, the group successfully selected a microbial strain that produced new natural products $(\mathbf{2 1 a} / \mathbf{b}$, Figure 4$){ }^{69}$ MS-based metabolomics approaches are now more commonly used to aid natural product discovery. ${ }^{96-101}$ LC-MS data is easily matched against databases; however, if a compound is a 'hit' that does not match the database, LC-MS data does not provide any information about the compound except for its molecular mass. More recently, molecular networking based on MS/MS fragmentation patterns that allows assignment of structural classes to unknown compounds has gained a lot of popularity. ${ }^{102,103}$

In recent years, NMR advancement led to faster acquisition through stronger magnets and made this technique more powerful and applicable for metabolomics studies. ${ }^{82,104}$ Yet, only few natural products studies have used NMR-based metabolomics as a dereplication tool. Chen and co-workers ${ }^{101}$ used ${ }^{1} \mathrm{H}$-NMR as well as LC-MS data for metabolomic profiling of spongeassociated actinomycetes. Thereby, ${ }^{1} \mathrm{H}-\mathrm{NMR}$ data of 64 microbial isolates was binned into 0.01 ppm integral regions and the resulting peaks were analysed via PCA. Comparison to LC-MS PCA revealed some overlap between the two analytical methods, which both identified a Streptomyces sp. to contain unique chemistry. ${ }^{101}$ Schroeder and his co-workers used differential analysis of double quantum filtered correlation spectroscopy (DOF-COSY) spectra, which they graphically analysed based on stacking of spectral bitmaps to identify of unique peaks. ${ }^{105}$ This was followed by detailed analysis of the unique signals and their corresponding spin-systems, which for a number of compounds was sufficient for their elucidation. ${ }^{105}$ For more complex structures, additional HSQC, HMBC, and NOESY spectra were acquired, which identified two new natural products. ${ }^{105}$ 


\subsubsection{One strain many compounds (OSMAC) approach}

Whole genome sequencing has revealed the true genetic potential of microorganisms; however, only a fraction of the biosynthetic genes are transcribed under laboratory conditions and many biosynthetic genes remain silent. ${ }^{81,106}$ Considering the functions of natural products in microbes, it is assumed that every natural product is a result of interactions of the organism with its environment. ${ }^{107,108}$ Manipulation of culture conditions with chemical or physical elicitors can exert stresses on the microbial culture and as a result lead to enhanced production of secondary metabolites. In 2002, Bode and co-workers ${ }^{109}$ reported that through small changes such as media composition, aeration, culture vessel or the addition of enzyme inhibitors, large effects on the secondary metabolite production could be observed. Twenty different metabolites could be isolated from just one strain and they gave this approach the term "one stain - many compounds (OSMAC)" ${ }^{109}$ Nowadays, this approach is common practice and has resulted in the isolation of natural products with enhanced chemical diversity.

\subsubsection{Elicitation of the microbial metabolome: community cultures}

In the environment, microorganisms usually live in diverse microbial communities. Interand intraspecific interactions of microorganisms may stimulate enhanced natural product synthesis. ${ }^{107}$ The specific mechanisms of these interactions are not fully resolved, although four different mechanisms have been proposed by Abdelmohsen and co-workers. ${ }^{110}$ Metabolite synthesis may be triggered through physical cell to cell interactions, small molecule mediated interactions, enzymes produced by one species that activate the metabolite precursor of another species. Alternatively, metabolite production could be made possible through gene transfer between two different species. ${ }^{110}$ Community cultures (co-cultures) of two or more different microorganisms intends to mimic such interactions in the laboratory. ${ }^{106}$ Several studies have exploited co-cultivations on solid agar or mixed liquid fermentations to induce synthesis of bioactive natural products that are not expressed in standard pure cultures. ${ }^{111-114}$ Cueto and coworkers ${ }^{114}$ demonstrated that mixed fermentations stimulated the production of a new compound, pestalone (27, Figure 7), in a marine Pestalotia species when co-cultured with an unidentified antibiotic-resistant marine bacterium. Pestalone, which is active against Staphylococcus aureus and Enterococcus faecium, was not detected in monocultures of the Pestalotia strain. ${ }^{114}$ While these techniques allow interactions between different members of the cultured communities that could trigger the production of promising lead structures for biomedical research, co-cultures are very dynamic and therefore difficult to reproduce. Furthermore, this technique still does not provide other variables of the source environment that in nature stimulate the production of secondary metabolites. ${ }^{106}$ 


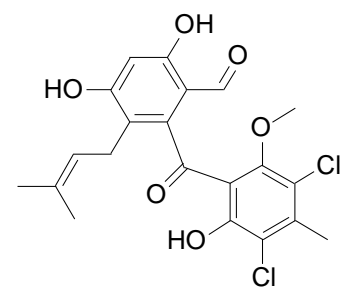

27

Figure 7. Pestalone (27), a new natural product triggered through co-cultivation

\subsection{Bioactivity target: the malaria parasite}

Malaria is a vector-borne infectious disease, caused by a protozoan parasite from the genus Plasmodium, with Plasmodium falciparum causing the most severe form of human malaria. The malaria parasite was responsible for almost half a million human deaths in 2015 mostly from developing countries of Africa, Latin America and South-East Asia. ${ }^{115}$ Humans contract malaria when a female Anopheles mosquito feeds on human blood. As the mosquito takes a blood meal, Plasmodium sporozoites in its saliva are simultaneously injected into the body, which then travel to the liver. ${ }^{116}$ Here, they bind to negatively charged sugars and asexually reproduce to give rise to thousands of merozoites that invade red blood cells, where the intraerythrocytic cycle begins. ${ }^{117}$ Within the red blood cells, merozoites develop into the early trophozoites, also known as the ring form. The mature trophozoites asexually multiply, ultimately forming 16 - 32 daughter merozoites. The production of daughter merozoites causes the red blood cell to rupture, releasing merozoites into the bloodstream. At this stage the disease manifests and the human host experiences symptoms of strong fevers and chills. ${ }^{118}$ Some merozoites then re-invade new red blood cells, repeating the cycle. Inside the red blood cells, a small portion of merozoites will form schitzonts, producing male and female gametocytes. The gametocytes are transmitted back to a mosquito as it takes a blood meal and there they fuse to form oocysts, which divide into sporozoites. These are then transferred to the mosquito's saliva glands and the life cycle of the parasite begins again once the mosquito feeds on a new human's blood (Figure 8). 


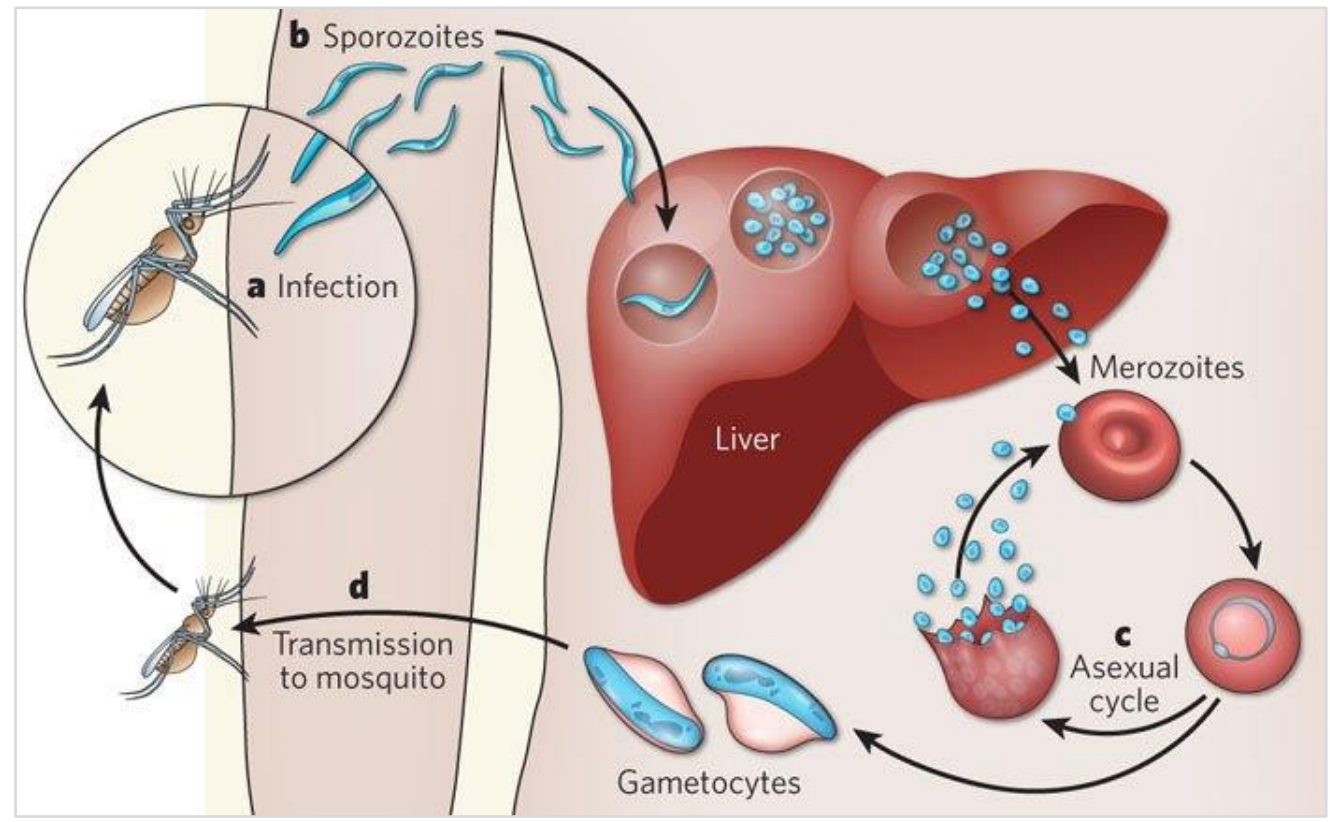

Figure 8. Life cycle of Plasmodium parasite ${ }^{118}$

The majority of drugs to treat malaria are natural products derived from plants. Quinine (28), chloroquine (29), artemisinin (30) and its derivatives, and antifolates are the most commonly used anti-plasmodial drugs, but resistance is emerging (Figure 9). All currently available antimalarial drugs have similar modes of actions: antifolate drugs target the folate biosynthesis pathway, quinoline-type compounds interfere with enzymatic conversion of toxic ferric haem into to a non-toxic form resulting in cell lysis of the parasite, and artemisinin blocks the production of phosphoinositol. ${ }^{119}$ Resistance to chloroquine, the cheapest and safest antimalarial drug, was first reported in Asia in 1957 and by 1970 chloroquine-resistance was spread throughout Asia, Africa, and South America. As a consequence, antifolate combination drugs were developed; however, the Plasmodium parasite developed resistance soon after their development. Artemisinin brought new hope to treat the disease and artemisinin mono and combination therapy proved effective against the parasite. In 2005, the World Health Organisation reported resistance to artemisinin at the Thailand-Cambodia boarder and advised withdrawal from monotherapy. The spread of artemisinin resistance presents a major threat to millions of people infected with malaria every year; new drugs with novel targets are urgently needed. 


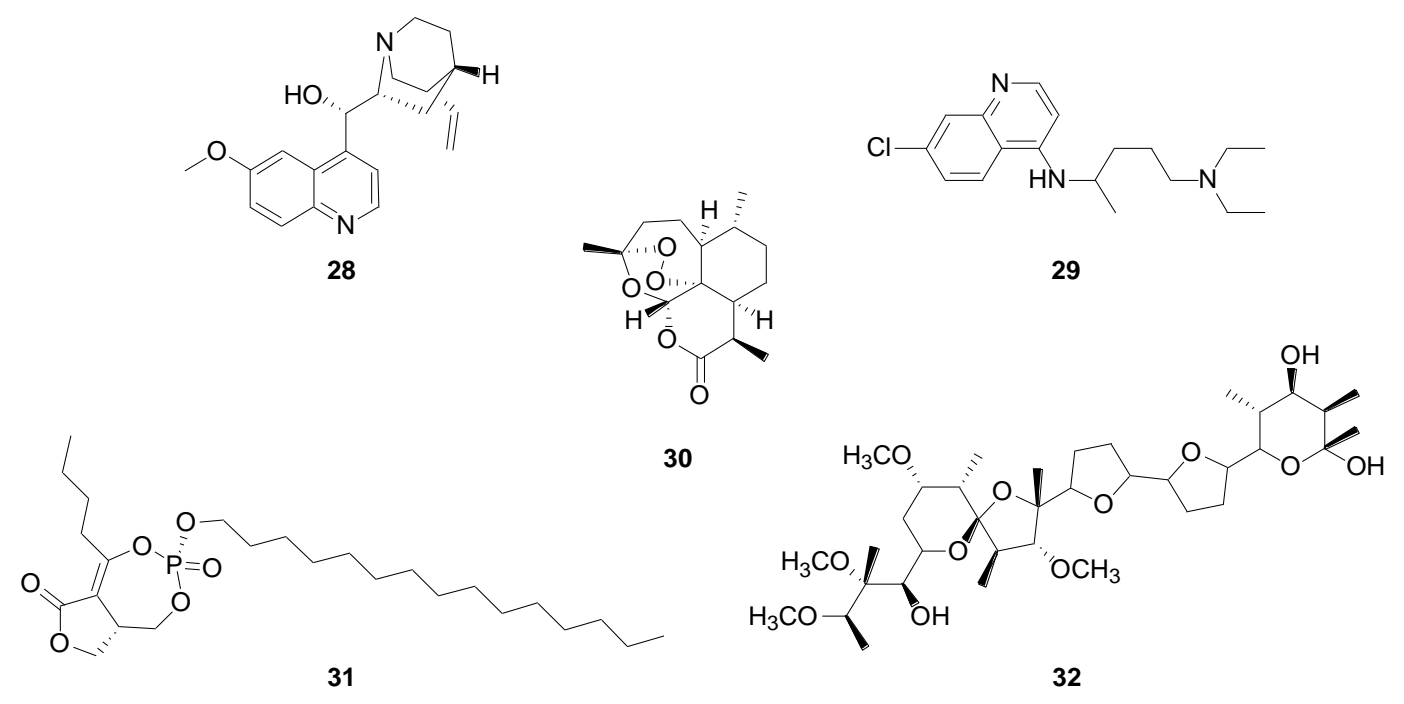

Figure 9. Anti-plasmodial natural products

Marine natural products have been a new focus on finding novel anti-plasmodial agents and also marine bacteria have yielded anti-plasmodial natural products. ${ }^{119}$ Salinosporamide A (9), from the marine actinomycete Salinospora tropica demonstrated potent activity against chloroquine-sensitive and resistant $P$. faliciparum clones. In a mouse model, growth inhibition of the parasite was observed at very low doses of $130 \mu \mathrm{g} / \mathrm{kg}{ }^{120}$ The long-chain bicyclic phosphotriester compounds salinipostins A (31) - K isolated from the marine-sediment derived Salinospora species exhibited anti-plasmodial activity, with salinipostin A having the lowest $\mathrm{EC}_{50}(50 \mathrm{nM})$ and high selectivity against mammalian cells. ${ }^{121}$ In a study by $\mathrm{Na}$ and coworkers $^{122}$, a marine Streptomyces sp. strain was identified that produced an anti-plasmodial polyether (32) $(0.145$ to $0.29 \mu \mathrm{M})$. Abdehlmosen and co-workers ${ }^{119}$ reviewed marine natural products and their drug potential that have biological activity against drug-resistant pathogens. It was found that the majority of the marine natural products, including several marine microbial compounds and invertebrate compounds that are likely to be synthesised by a microbial symbiont, exhibit drug-like properties as they adhered to the Lipinski's rule of five. Fewer antiplasmodial compounds showed good oral bioavailability; particularly the salinipostins were unfavourable due to their high number of rotatable bonds, thus these characteristics should be assessed thoroughly before pursuing developement. ${ }^{119}$ Nonetheless, the authors state that new discoveries of natural products from the marine environment, particular actinomycetes and sponges, bring new hope for the treatment of drug-resistant infections. 


\subsection{Overview and aims of the thesis}

The overarching aim of this thesis was to integrate and explore different biodiscovery approaches to maximise natural product discovery from marine actinomycetes (Figure 10). The thesis consists of seven chapters: a general introduction, five results chapters and a conclusion $\&$ outlook chapter. This thesis has been prepared in accordance with the Griffith University policy on inclusion of papers within a thesis. The results chapters $1-5$ are written in form of manuscripts in preparation (Chapters 3 - 5), submitted (Chapter 2) or that have been accepted (Chapter 6) in peer-reviewed academic journals. Each of the chapters can stand alone and therefore, there is some degree of repetition among the introductions, methods, and results of these chapters.

In the light of the above presented introduction, it has become apparent that the major issue of microbial natural product drug discovery is the high rediscovery of already known compounds. To account for this issue, this $\mathrm{PhD}$ project focused in the first section on the isolation of actinomycetes associated with three Australian marine ascidians, an underexplored ecological niche, in order tap into new bacterial and chemical diversity. Section 2 looks at diversifying microbial natural products for drug discovery. It was envisaged that a new innovative metabolomics strain selection approached should then be implemented to identify actinomycete strains within the established strain library that are more likely to produce new bioactive metabolites and exclude redundant strains to minimise repeated isolation of the same compounds. Furthermore, the effect of co-cultivation on the chemical diversity of microbial extracts was evaluated using the newly developed metabolomics approach. Classic natural product chemistry isolation and structure elucidation approaches were then implemented in Section 3 to characterise new anti-plasmodial actinomycete natural products (Figure 10). 


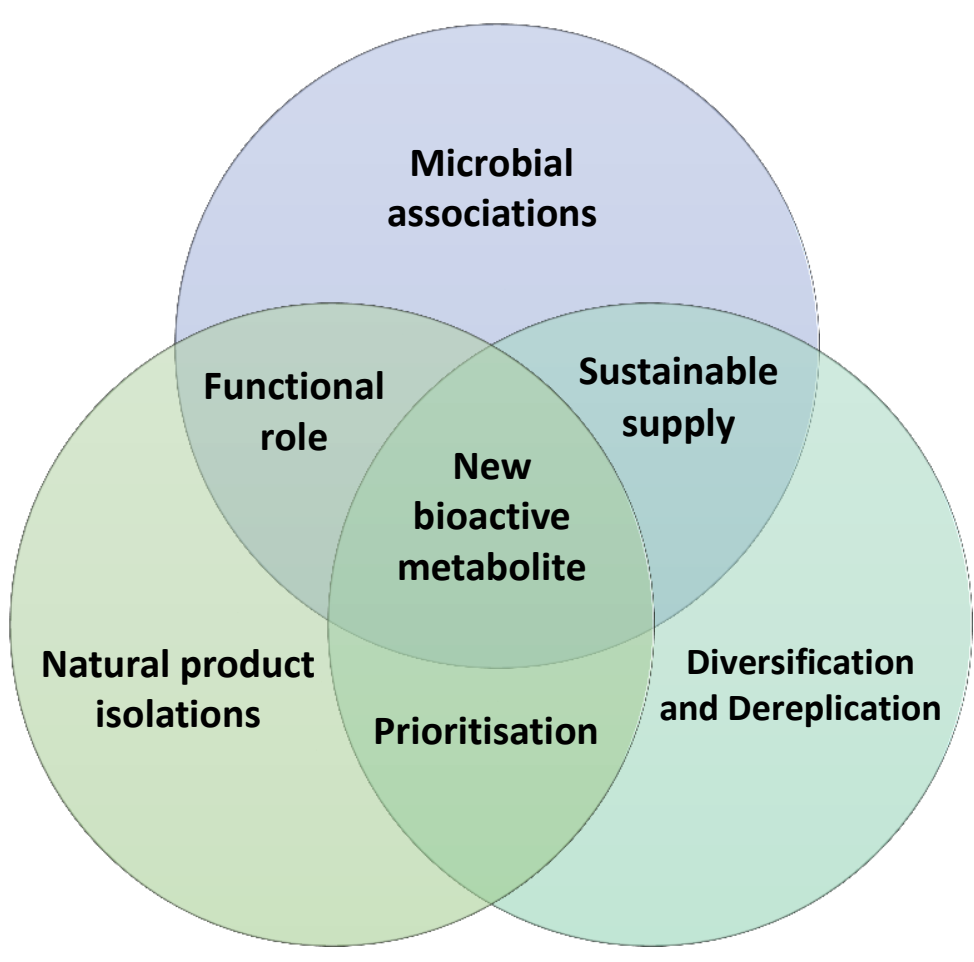

Figure 10. Integrated approaches to characterise new bioactive metabolites

The thesis specifically aims to make the following scientific contributions:

1. Establish an isolate library of ascidian-associated actinomycete strains and evaluate their chemical potential. (Chapter 2)

2. Develop a new dereplication/strain prioritisation approach that utilised twodimensional NMR profiling and anti-plasmodial activity data to identify microbial strains that have greater potential to produce anti-plasmodial secondary metabolites. (Chapter 3)

3. Enhance chemical diversity through co-culturing and identify changes in the actinomycete secondary metabolome through 2D NMR profiling. (Chapter 4)

4. Isolate and elucidate new anti-plasmodial peptide and polyketide natural products from selected marine actinomycete strains. (Chapters 5 and 6) 


\subsection{References}

(1) Firn, R. D.; Jones, C. G. Nat. Prod. Rep. 2003, 20, 382.

(2) Feher, M.; Schmidt, J. M. Chem. Inf. Comput. Sci. 2003, 43, 218.

(3) Butler, M. S. J. Nat. Prod. 2004, 67, 2141.

(4) Paul, V. J.; Puglisi, M. P.; Ritson-Williams, R. Nat. Prod. Rep. 2006, 23, 153.

(5) Hay, M. E. Ann. Rev. Mar. Sci. 2009, 1, 193.

(6) Mishra, B. B.; Tiwari, V. K. Eur. J. Med. Chem. 2011, 46, 4769.

(7) Newman, D. J.; Cragg, G. M. J. Nat. Prod. 2007, 70, 461.

(8) Cragg, G. M.; Grothaus, P. G.; Newman, D. J. J. Nat. Prod. 2014, 77, 703.

(9) Newman, D. J.; Cragg, G. M. J. Nat. Prod. 2016, 79, 629.

(10) Patridge, E.; Gareiss, P.; Kinch, M. S.; Hoyer, D. Drug Discovery Today 2016, 21, 204.

(11) Rouhi, A. M. Chem. Eng. News 2003, 81, 77.

(12) Li, J. W.-H.; Vederas, J. C. Science 2009, 325, 161.

(13) Kurtböke, D. İ; Grkovic, T.; Quinn, R. J. In Springer Handbook of Marine Biotechnology; Springer: 2015, p 663.

(14) Bhatnagar, I.; Kim, S.-K. Mar. Drugs 2010, 8, 2673.

(15) Ito, T.; Masubuchi, M. J. Antibiot. 2014, 67, 353.

(16) Knight, V.; Sanglier, J.-J.; DiTullio, D.; Braccili, S.; Bonner, P.; Waters, J.; Hughes, D.; Zhang, L. Appl. Microbiol. Biotechnol. 2003, 62, 446.

(17) Schaal, K. P.; Yassin, A. F.; Stackebrandt, E. In The prokaryotes; Springer: 2006, p 430.

(18) Waksman, S. A. Actinomycetes; LWW, 1950; Vol. 70.

(19) Li, Q.; Chen, X.; Jiang, Y.; Jiang, C. In Actinobacteria - Basics and Biotechnological Applications; Dhanasekaran, D., Jiang, Y., Eds.; InTech: Rijeka, 2016, p Ch. 03.

(20) Santhanam, R.; Rong, X.; Huang, Y.; Andrews, B. A.; Asenjo, J. A.; Goodfellow, M. Antonie Van Leeuwenhoek 2013, 103, 367.

(21) Luo, Y.; Xiao, J.; Wang, Y.; Xu, J.; Xie, S.; Xu, J. Int. J. Syst. Evol. Microbiol. 2011, 61, 2712.

(22) Passari, A. K.; Mishra, V. K.; Saikia, R.; Gupta, V. K.; Singh, B. P. Front. Microbiol. 2015, 6 .

(23) Kurtböke, D. İ.; French, J. R.; Hayes, R. A.; Quinn, R. J. In Biotechnological Applications of Biodiversity; Springer: 2014, $\mathrm{p} 111$.

(24) Abdelmohsen, U. R.; Pimentel-Elardo, S. M.; Hanora, A.; Radwan, M.; Abou-El-Ela, S. H.; Ahmed, S.; Hentschel, U. Mar. Drugs 2010, 8, 399.

(25) Mahmoud, H. M.; Kalendar, A. A. Front. Microbiol. 2016, 7.

(26) Berdy, J. J. Antibiot. 2005, 1.

(27) Fenical, W. Chem. Rev. 1993, 93, 1673.

(28) Jensen, P. R.; Gontang, E.; Mafnas, C.; Mincer, T. J.; Fenical, W. Environ. Microbiol. 2005, 7, 1039.

(29) Fenical, W.; Jensen, P. R. Nat. Chem. Biol. 2006, 2, 666.

(30) Abdelmohsen, U. R.; Bayer, K.; Hentschel, U. Nat. Prod. Rep. 2014, 31, 381. 
(31) Hameş-Kocabaş, E. E.; Ataç, U. J. Microbiol. Methods 2012, 88, 342.

(32) Subramani, R.; Aalbersberg, W. Appl. Microbiol. Biotechnol. 2013, 97, 9291.

(33) Olano, C.; Méndez, C.; Salas, J. A. Mar. Drugs 2009, 7, 210.

(34) Kwon, H. C.; Kauffman, C. A.; Jensen, P. R.; Fenical, W. J. Am. Chem. Soc. 2006, 128, 1622.

(35) Asolkar, R. N.; Jensen, P. R.; Kauffman, C. A.; Fenical, W. J. Nat. Prod. 2006, 69, 1756.

(36) Durán, N.; Justo, G. Z.; Ferreira, C. V.; Melo, P. S.; Cordi, L.; Martins, D. Biotechnol. Appl. Biochem. 2007, 48, 127.

(37) Feling, R. H.; Buchanan, G. O.; Mincer, T. J.; Kauffman, C. A.; Jensen, P. R.; Fenical, W. Angew. Chem., Int. Ed. 2003, 42, 355.

(38) Fenical, W.; Jensen, P. R.; Palladino, M. A.; Lam, K. S.; Lloyd, G. K.; Potts, B. C. Bioorg. Med. Chem. 2009, 17, 2175.

(39) Newman, D. J.; Cragg, G. M. Mar. Drugs 2014, 12, 255.

(40) Badros, A.; Singh, Z.; Dhakal, B.; Kwok, Y.; MacLaren, A.; Richardson, P.; Trikha, M.; Hari, P. Brit. J. Haematol. 2017, 177, 221.

(41) Nett, M.; König, G. M. Nat. Prod. Rep. 2007, 24, 1245.

(42) Udwary, D. W.; Zeigler, L.; Asolkar, R. N.; Singan, V.; Lapidus, A.; Fenical, W.; Jensen, P. R.; Moore, B. S. Proc. Natl. Acad. Sci. U. S. A. 2007, 104, 10376.

(43) Demain, A. L. J. Ind. Microbiol. Biotechnol. 2014, 41, 185.

(44) Williams, P. G.; Asolkar, R. N.; Kondratyuk, T.; Pezzuto, J. M.; Jensen, P. R.; Fenical, W. J. Nat. Prod. 2007, 70, 83.

(45) Asolkar, R. N.; Freel, K. C.; Jensen, P. R.; Fenical, W.; Kondratyuk, T. P.; Park, E.-J.; Pezzuto, J. M. J. Nat. Prod. 2008, 72, 396.

(46) Wu, M.-C.; Law, B.; Wilkinson, B.; Micklefield, J. Curr. Opin. Biotechnol. 2012, 23, 931.

(47) Zotchev, S. B. J. Biotechnol. 2012, 158, 168.

(48) Schmidt, E. W.; Donia, M. S.; McIntosh, J. A.; Fricke, W. F.; Ravel, J. J. Nat. Prod. 2012, 75, 295.

(49) Crawford, J. M.; Clardy, J. Chem. Commun.2011, 47, 7559.

(50) Chen, L.; Fu, C.; Wang, G. Symbiosis 2016, 1.

(51) Long, P. F.; Dunlap, W. C.; Battershill, C. N.; Jaspars, M. ChemBioChem 2005, 6, 1760.

(52) Schreiber, L.; Kjeldsen, K. U.; Funch, P.; Jensen, J.; Obst, M.; López-Legentil, S.; Schramm, A. Front. Microbiol. 2016, 7.

(53) Valliappan, K.; Sun, W.; Li, Z. Appl. Microbiol. Biotechnol. 2014, 98, 7365.

(54) Behrendt, L.; Larkum, A. W.; Trampe, E.; Norman, A.; Sørensen, S. J.; Kühl, M. ISME J. 2012, 6, 1222.

(55) Tianero, M. D. B.; Kwan, J. C.; Wyche, T. P.; Presson, A. P.; Koch, M.; Barrows, L. R.; Bugni, T. S.; Schmidt, E. W. ISME J. 2014.

(56) Steinert, G.; Taylor, M. W.; Schupp, P. J. J. Mar. Biotechnol. 2015, 17, 377.

(57) Meziti, A.; Kormas, K. A.; Pancucci-Papadopoulou, M.-A.; Thessalou-Legaki, M. Russ. J. Mar. Biol. 2007, 33, 84. 
(58) McDonald, L. A.; Capson, T. L.; Krishnamurthy, G.; Ding, W.-D.; Ellestad, G. A.; Bernan, V. S.; Maiese, W. M.; Lassota, P.; Discafani, C.; Kramer, R. A. J. Am. Chem. Soc. 1996, 118, 10898.

(59) Lee, M. D.; Ellestad, G. A.; Borders, D. B. Acc. Chem. Res. 1991, 24, 235.

(60) Golik, J.; Clardy, J.; Dubay, G.; Groenewold, G.; Kawaguchi, H.; Konishi, M.; Krishnan, B.; Ohkuma, H.; Saitoh, K.; Doyle, T. W. J. Am. Chem. Soc. 1987, 109, 3461.

(61) Horton, P.; Longley, R.; McConnell, O.; Ballas, L. Cell. Mol. Life Sci. 1994, 50, 843.

(62) Andréo, M. A.; Jimenez, P. C.; Siebra, J. B.; Costa-Lotufo, L. V.; Vessecchi, R.; Niehues, M.; Lopes, J. L.; Lopes, N. P. J. Braz. Chem. Soc.2012, 23, 335.

(63) He, H.; Ding, W.-D.; Bernan, V. S.; Richardson, A. D.; Ireland, C. M.; Greenstein, M.; Ellestad, G. A.; Carter, G. T. J. Am. Chem. Soc. 2001, 123, 5362.

(64) Jimenez, P. C.; Ferreira, E. G.; Araújo, L. A.; Guimarães, L. A.; Sousa, T. S.; Pessoa, O. D. L.; Lotufo, T. M.; Costa-Lotufo, L. V. Lat. Am. J. Aquat. Res. 2013, 41, 335.

(65) Li, A.; Piel, J. Chem. Biol. 2002, 9, 1017.

(66) Hayakawa, Y.; Shirasaki, S.; Shiba, S.; Kawasaki, T.; Matsuo, Y.; Adachi, K.; Shizuri, Y. J. Antibiot. 2007, 60, 196.

(67) Charan, R. D.; Schlingmann, G.; Janso, J.; Bernan, V.; Feng, X.; Carter, G. T. J. Nat. Prod. 2004, 67, 1431.

(68) Wyche, T. P.; Hou, Y.; Vazquez-Rivera, E.; Braun, D.; Bugni, T. S. J. Nat. Prod. 2012, 75,735 .

(69) Zhang, Y.; Adnani, N.; Braun, D. R.; Ellis, G. A.; Barns, K. J.; Parker-Nance, S.; Guzei, I. A.; Bugni, T. S. J. Nat. Prod. 2016, 79, 2968.

(70) Radjasa, O. K.; Vaske, Y. M.; Navarro, G.; Vervoort, H. C.; Tenney, K.; Linington, R. G.; Crews, P. Bioorg. Med. Chem. 2011, 19, 6658.

(71) Cuevas, C.; Francesch, A. Nat. Prod. Rep. 2009, 26, 322.

(72) Martins, A.; Vieira, H.; Gaspar, H.; Santos, S. Mar. Drugs 2014, 12, 1066.

(73) Leal, M. C.; Sheridan, C.; Osinga, R.; Dionísio, G.; Rocha, R. J. M.; Silva, B.; Rosa, R.; Calado, R. Mar. Drugs 2014, 12, 3929.

(74) Dunlap, W. C.; Jaspars, M.; Hranueli, D.; Battershill, C. N.; Peric-Concha, N.; Zucko, J.; Wright, S. H.; Long, P. F. Curr. Med. Chem. 2006, 13, 697.

(75) Ikeda, Y.; Shimada, Y.; Honjo, K.; Okumoto, T.; Munakata, T. J. Antibiot. 1983, 36, 1290 .

(76) Arai, T.; Takahashi, K.; Ishiguro, K.; Yazawa, K. J. Antibiot. 1980, 33, 951.

(77) Endo, A.; Yanagisawa, A.; Abe, M.; Tohma, S.; Kan, T.; Fukuyama, T. J. Am. Chem. Soc. 2002, 124, 6552 .

(78) Newman, D. J. Pharmacol. Ther. 2016, 162, 1.

(79) Aicher, T. D.; Buszek, K. R.; Fang, F. G.; Forsyth, C. J.; Jung, S. H.; Kishi, Y.; Matelich, M. C.; Scola, P. M.; Spero, D. M.; Yoon, S. K. J. Am. Chem. Soc. 1992, 114, 3162.

(80) Towle, M. J.; Salvato, K. A.; Budrow, J.; Wels, B. F.; Kuznetsov, G.; Aalfs, K. K.; Welsh, S.; Zheng, W.; Seletsky, B. M.; Palme, M. H. Cancer Res. 2001, 61, 1013.

(81) Wilkinson, B.; Micklefield, J. Nat. Chem. Biol. 2007, 3, 379.

(82) Molinski, T. F.; Org. Lett. 2014, 16, 3849.

(83) Gaudêncio, S. P.; Pereira, F. Nat. Prod. Rep. 2015, 32, 779. 
(84) Hildebrand, M.; Waggoner, L. E.; Lim, G. E.; Sharp, K. H.; Ridley, C. P.; Haygood, M. G. Nat. Prod. Rep. 2004, 21, 122.

(85) Altschul, S. F.; Gish, W.; Miller, W.; Myers, E. W.; Lipman, D. J. J. Mol. Biol. 1990, $215,403$.

(86) Ritacco, F.; Haltli, B.; Janso, J.; Greenstein, M.; Bernan, V. J. Ind. Microbiol. Biotechnol. 2003, 30, 472.

(87) Antonio, J.; Molinski, T. F. J. Nat. Prod. 1993, 56, 54.

(88) Zani, C. L.; Carroll, A. R. J. Nat. Prod. 2017.

(89) Munro, M.; Blunt, J. University of Canterbury, New Zealand 2009.

(90) Laatsch, H. AntiBase 2012: the natural compound identifier; Wiley-Vch, 2011.

(91) Whittle, M.; Willett, P.; Klaffke, W.; van Noort, P. J. Chem. Inf. Model. 2003, 43, 449.

(92) Kurita, K. L.; Linington, R. G. J. Nat. Prod. 2015, 78, 587.

(93) Rochfort, S. J. Nat. Prod. 2005, 68, 1813.

(94) Grootveld, M. In Metabolic Profiling: Disease and Xenobiotics; Royal Society of Chemistry, 2012.

(95) Hou, Y.; Braun, D. R.; Michel, C. R.; Klassen, J. L.; Adnani, N.; Wyche, T. P.; Bugni, T. S. Anal. Chem. 2012, 84, 4277.

(96) Ito, T.; Odake, T.; Katoh, H.; Yamaguchi, Y.; Aoki, M. J. Nat. Prod. 2011, 74, 983.

(97) Macintyre, L.; Zhang, T.; Viegelmann, C.; Martinez, I. J.; Cheng, C.; Dowdells, C.; Abdelmohsen, U. R.; Gernert, C.; Hentschel, U.; Edrada-Ebel, R. Mar. Drugs 2014, 12, 3416 .

(98) Nielsen, K. F.; Månsson, M.; Rank, C.; Frisvad, J. C.; Larsen, T. O. J. Nat. Prod. 2011, 74, 2338.

(99) Bose, U.; Hewavitharana, A. K.; Ng, Y. K.; Shaw, P. N.; Fuerst, J. A.; Hodson, M. P. Mar. Drugs 2015, 13, 249.

(100) Abdelmohsen, U. R.; Cheng, C.; Viegelmann, C.; Zhang, T.; Grkovic, T.; Ahmed, S.; Quinn, R. J.; Hentschel, U.; Edrada-Ebel, R. Mar. Drugs 2014, 12, 1220.

(101) Cheng, C.; MacIntyre, L.; Abdelmohsen, U. R.; Horn, H.; Polymenakou, P. N.; EdradaEbel, R.; Hentschel, U. PloS one 2015, 10, e0138528.

(102) Watrous, J.; Roach, P.; Alexandrov, T.; Heath, B. S.; Yang, J. Y.; Kersten, R. D.; van der Voort, M.; Pogliano, K.; Gross, H.; Raaijmakers, J. M. Proc. Natl. Acad. Sci. U. S. A. 2012, 109, E1743.

(103) Yang, J. Y.; Sanchez, L. M.; Rath, C. M.; Liu, X.; Boudreau, P. D.; Bruns, N.; Glukhov, E.; Wodtke, A.; De Felicio, R.; Fenner, A. J. Nat. Prod. 2013, 76, 1686.

(104) Halouska, S.; Zhang, B.; Gaupp, R.; Lei, S.; Snell, E.; Fenton, R. J.; Barletta, R. G.; Somerville, G. A.; Powers, R. J. Integr. OMICS 2013, 3, 120.

(105) Schroeder, F. C.; Gibson, D. M.; Churchill, A. C.; Sojikul, P.; Wursthorn, E. J.; Krasnoff, S. B.; Clardy, J. Angew. Chem., Int. Ed. 2007, 46, 901.

(106) Marmann, A.; Aly, A. H.; Lin, W.; Wang, B.; Proksch, P. Mar. Drugs 2014, 12, 1043.

(107) Scherlach, K.; Hertweck, C. Org. Biomol. Chem. 2009, 7, 1753.

(108) Alain, K.; Querellou, J. Extremophiles 2009, 13, 583.

(109) Bode, H. B.; Bethe, B.; Höfs, R.; Zeeck, A. ChemBioChem 2002, 3, 619. 
(110) Abdelmohsen, U. R.; Grkovic, T.; Balasubramanian, S.; Kamel, M. S.; Quinn, R. J.; Hentschel, U. Biotechnol. Adv. 2015, 33, 798.

(111) Traxler, M. F.; Watrous, J. D.; Alexandrov, T.; Dorrestein, P. C.; Kolter, R. MBio 2013, 4, e00459.

(112) Slattery, M.; Rajbhandari, I.; Wesson, K. Microb. Ecol. 2001, 41, 90.

(113) Zhu, F.; Chen, G.; Chen, X.; Huang, M.; Wan, X. Chem. Nat. Compd. 2011, 47, 767.

(114) Cueto, M.; Jensen, P. R.; Kauffman, C.; Fenical, W.; Lobkovsky, E.; Clardy, J. J. Nat. Prod. 2001, 64, 1444.

(115) World Health Organization, World malaria report 2016; Available online: http://www.who.int/malaria/publications/world-malaria-report-2016/report/en/

(116) Tuteja, R. FEBS J. 2007, 274, 4670.

(117) Shahinas, D.; Folefoc, A.; Pillai, D. R. Pathogens 2013, 2, 33.

(118) Michalakis, Y.; Renaud, F. Nature 2009, 462, 298.

(119) Abdelmohsen, U. R.; Balasubramanian, S.; Oelschlaeger, T. A.; Grkovic, T.; Pham, N. B.; Quinn, R. J.; Hentschel, U. Lancet Infect. Dis. 2016.

(120) Prudhomme, J.; McDaniel, E.; Ponts, N.; Bertani, S.; Fenical, W.; Jensen, P.; Le Roch, K. PloS one 2008, 3, e2335.

(121) Schulze, C. J.; Navarro, G.; Ebert, D.; DeRisi, J.; Linington, R. G. J. Org. Chem. 2015, 80, 1312.

(122) Na, M.; Meujo, D. A.; Kevin, D.; Hamann, M. T.; Anderson, M.; Hill, R. T. Tetrahedron Lett. 2008, 49, 6282. 


\section{SECTION 1}

\section{Untapped ecological niche: Ascidian-associated actinomycetes}

A powerful approach to enhance chemical diversity and discover novel natural products is to explore new sources of organisms that have not previously been investigated. Historically, microorganisms from the terrestrial environment have been a very successful source for biodiscovery and numerous compounds derived from terrestrial microorganisms have become marketed drugs. On the contrary, the marine environment contains an incredibly rich and vast biodiversity, most of which still remains to be uncovered. In Chapter 2 of this thesis, I set out to explore actinomycetes associated with the tree Australian ascidians, Symplegma rubra, Aplidium solidium and Polyclinum vasculosum, collected from Hastings Point in Northern New South Wales, Australia. This region, unlike the Great Barrier Reef, has not received a lot of attention for biodiscovery. Yet, it is shaped by intermixing of tropical waters coming from the north and temperate waters from the south making it a unique target for biodiscovery. Furthermore, the microbial communities associated with the three chosen ascidian species have not previously investigated. Through this work I wanted to answer the following questions: Are there any actinomycetes associated with these three ascidian species? What families of actinomycetes are associated with these ascidians and can they be cultured? Are the ascidian-associated actinomycetes a good source for natural product drug discovery and biotechnology? 


\section{CHAPTER 2 - Taxonomic and metabolite diversity of actinomycetes associated with three Australian ascidians}

\section{STATEMENT OF CONTRIBUTION TO CO-AUTHORED SUBMITTED PAPER}

This chapter has been submitted as a co-authored paper to the journal Diversity. The bibliographic details of the co-authored paper, including all authors, are:

Buedenbender, L., Carroll, A.R., Ekins, M., Kurtböke, D.I., Taxonomic and metabolite diversity of actinomycetes associated with three Australian ascidians, Diversity, 2017, under review

L.Buedenbender designed the experiments; organised the sampling event, performed the experiments, conducted molecular work, analysed the microbiome, as well as microbiological and chemical data and wrote the manuscript; D. I. Kurtböke and A. R. Carroll are the supervisors of the $\mathrm{PhD}$ project and provided advice on the research design and feedback. M. Ekins identified the ascidian specimens to species level.

(Signed)

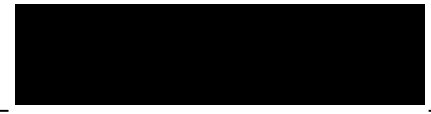
(Date) $\underline{01 / 09 / 2017}$

Larissa Buedenbender

(Countersigned) (Date) $\underline{31 / 08 / 2017}$

Corresponding author of paper: D. Ipek Kurtböke

(Countersigned)

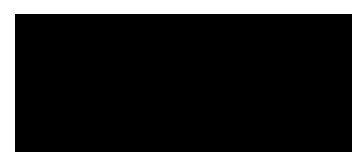
(Date) $\underline{31 / 08 / 2017}$

Supervisor: Anthony R. Carroll 


\subsection{Abstract}

Actinomycetes are known to be the most prolific producers of biologically active metabolites. Here, we investigated the host species-specificity and related secondary metabolites of actinomycetes associated with three different Australian ascidians, namely Symplegma rubra, Aplidium solidum and Polyclinum vasculosum. Results indicated that while isolates from the genera Streptomyces and Micromonospora were highly diverse in the ascidian samples, only two culturable actinomycete OTUs overlapped between all of the ascidians, pointing to some degree of host species-specificity of the isolates and selective acquisition of microbial associates by the host from the surrounding environment. LC-MS/MS profiling of extracts obtained from the ascidians and their actinomycete associates revealed many overlapping ions between hosts and actinomycetes indicating that these compounds were likely to be synthesised by the microbial associates. Laboratory cultures of the actinomycetes displayed even more diverse metabolomes than those of their ascidian hosts; thus, making ascidian-associated actinomycetes an excellent target for natural product drug discovery and biotechnology.

Keywords: Actinobacteria, Actinomycetes, Actinomycetales; marine microbial diversity; ascidian-associated actinomycetes; secondary metabolites

\subsection{Introduction}

Ascidians (phylum: Chordata, sub-phylum: Tunicata) are soft-bodied, sessile, filter-feeding marine invertebrates that often rely on secondary metabolites for their defence. ${ }^{1-3}$ These compounds often show potent biological activity and thus have been recognised for their pharmaceutical and biotechnological potential. Some striking examples include ecteinascidin marketed as Yondelis®, and others undergoing clinical trials are plitidepsin and lurbinectedin. ${ }^{4}$ Even though ascidians have immense potential for the discovery of bioactive metabolites, sustainable supply of these bioactive molecules from wild harvesting of these animals is difficult. This is because ascidians often live in large colonies of thousands of individuals, but are only a few millimetres thick.

Increasing evidence has shown that associated bacteria are the true producers of many of the ascidian's bioactive metabolites. ${ }^{5,6}$ Therefore, isolation of microbial associates provides a sustainable source of supply for the development of novel pharmaceutical drug leads, as it permits fermentation-based production of natural products rather than the harvest of enormous amounts of marine invertebrate biomass. ${ }^{7,8}$ The composition of the microbiome associated with the ascidian host has been shown to be species-specific, as well as influenced by the physiological environmental at the geographical location. ${ }^{9,10}$ Currents, preceding weather events, 
and vicinity to urbanisations are some of the factors that can impact acquisition of the microbial associates by the invertebrate host during filter feeding. These factors ought to be considered when obtaining samples in order to identify the specificity of ascidian-associated bacteria that are producers of diverse bioactive metabolites found in the host. ${ }^{11}$

Even though ascidians and microorganisms are known to associate, the true nature of the relationship between the bacteria and the ascidian host remain mostly unresolved. Obligate symbiosis has been confirmed between the alpha-proteobacterium, Candidatus Endoecteinascidia faulkneri and the ascidian Lissoclinum patella. The symbiont lives intracellularly in the blood cells of the host and is vertically transmitted over generations; as a result, the bacterium has lost its original shape and the genome has degraded to solely produce bioactive secondary metabolites. ${ }^{12,13}$ Symbiotic relationships have also been defined for the cyanobacterium, Prochloron didemni. However, in this case the symbionts are found extracellularly and the bacterium has been associated with nutrition of fixed carbon and the recycling of nitrogen. ${ }^{14,15}$ Additionally, genes responsible for the synthesis of highly toxic cyanobactins were identified in the $P$. didemni genome, but could not be found in the didemnid ascidian genome. ${ }^{5,16,17}$ Chemistry of didemnid ascidians often varies between animals; therefore, an ascidian species-specific combination of horizontal and vertical transmission of symbiotic bacteria has been proposed. ${ }^{16,18}$

Actinobacteria have been reported in marine environments and in associations with ascidians. ${ }^{19-21}$ Current intraclass relatedness of the class Actinobacteria reveals the presence of nine orders (http://www.bacterio.net/Actinomycetales.html, accessed 14-08-2017). Accordingly, "Actinobacteria" in this paper will only refer to the "actinomycetes" covering the members of the order "Actinomycetales" in the historical sense. It will also include the newly created orders Frankiales, Geodermatophiales, Kineosporiales and Micrococcales (http://www.bacterio.net/Actinomycetales.html, accessed 14-08-2017) as they were part of this order until recently. ${ }^{22}$

Actinomycetes are known as prolific producers of bioactive metabolites, and several bioactive secondary metabolites have been isolated from ascidian-associated actinomycetes. ${ }^{23-27}$ However, most studies are inconclusive in relation to revealing any functional role of ascidianassociated actinomycetes. In most cases, the actinomycete metabolites could not be detected in their ascidian host extracts, leading to the conclusion that actinomycetes do not play an important role in secondary metabolism symbiosis and their associations to ascidians might be of facultative nature where actinomycetes are horizontally transmitted from the environment. ${ }^{6}$ In contrast to these findings, several staurosporine derivatives, a structure class known to be synthesised by terrestrial and marine actinomycetes, have repeatedly been isolated from 
Eudistoma ascidians, ${ }^{28}$ pointing towards a functional role of staurosporine production by a symbiotic actinomycetes strain. ${ }^{29}$

The Polyclinidae and Styelidae are two of three ascidian families from which the majority of bioactive natural products have so far been isolated, many of which are likely to be of microbial origin. ${ }^{30}$ Yet, few attempts have been made to investigate their microbial associates. Herein, we selected Aplidium solidum and Polyclinum vasculosum both belonging to the same taxonomic family Polyclinidae, which are abundant species at Hastings Point in Northern New South Wales, Australia, as well as Symplegma rubra (Styelidae) from the same marine environment to explore species-specificity of their associated actinomycete diversity that had not previously been examined. As it is suggested that actinomycetes are horizontally acquired from the environment, we hypothesised that consequently their diversity should greatly overlap between the three different species sampled at the same location. In order to evaluate this hypothesis, we initially examined the overall microbial diversity associated with the three Australian ascidians to identify the presence of actinomycetes based on a culture-independent approach. We then focused our culturing efforts to isolate actinomycetes and conducted molecular networking of actinomycete extracts as well as ascidian extracts to depict their metabolic diversity and identify metabolites of the ascidian metabolome that are potentially synthesised by an actinomycete associate.

\subsection{Materials and Methods}

\subsubsection{Site description and ascidian collection}

Hastings Point is a rocky shore habitat that receives warm waters from the East Australian Current containing tropical marine invertebrate larvae as well as more temperate waters cycling back from the South. ${ }^{31}$ The intermixing of cold and warm waters results in a rich and unique biodiversity of marine invertebrates, predominantly ascidians, making the location a prime target for biodiscovery. For this study, three different ascidian specimens were collected. Aplidium solidum (QM G335842) and Polyclinum vasculosum (QM G335843), both belonging to the same taxonomic family Polyclinidae, were obtained on 13 July 2015 from rock pools at depths between $1-2 \mathrm{~m}$ at Hastings Point, New South Wales, Australia (28 $\left.22^{\prime} \mathrm{S}, 153^{\circ} 35^{\prime} \mathrm{E}\right)$. Symplegma rubra (QM G335841), family Styelidae, was collected by snorkel at a depth of $6 \mathrm{~m}$ at a rocky reef approximately 200 metres offshore from Hastings Point headland (Site 1, Figure 1a). 
All three ascidians are colonial species with microsize tunics (approximately $1-4 \mathrm{~mm}$ thick). The samples were collected at low tide under a Scientific Collection Permit (Permit No: P09/0031-3.0) obtained from the New South Wales Department of Primary Industries. Ascidian samples were placed into separate plastic zip lock bags containing seawater and were transported to the laboratory on ice within 2 hours of sampling. About $1-16 \mathrm{~g}$ of ascidian tissue was exhaustively extracted with methanol to obtain a crude chemical profile of each specimen. Preserved ascidian specimens were deposited at the Queensland Museum, Brisbane (http://www.qm.qld.gov.au/) where they were identified taxonomically at the species level.

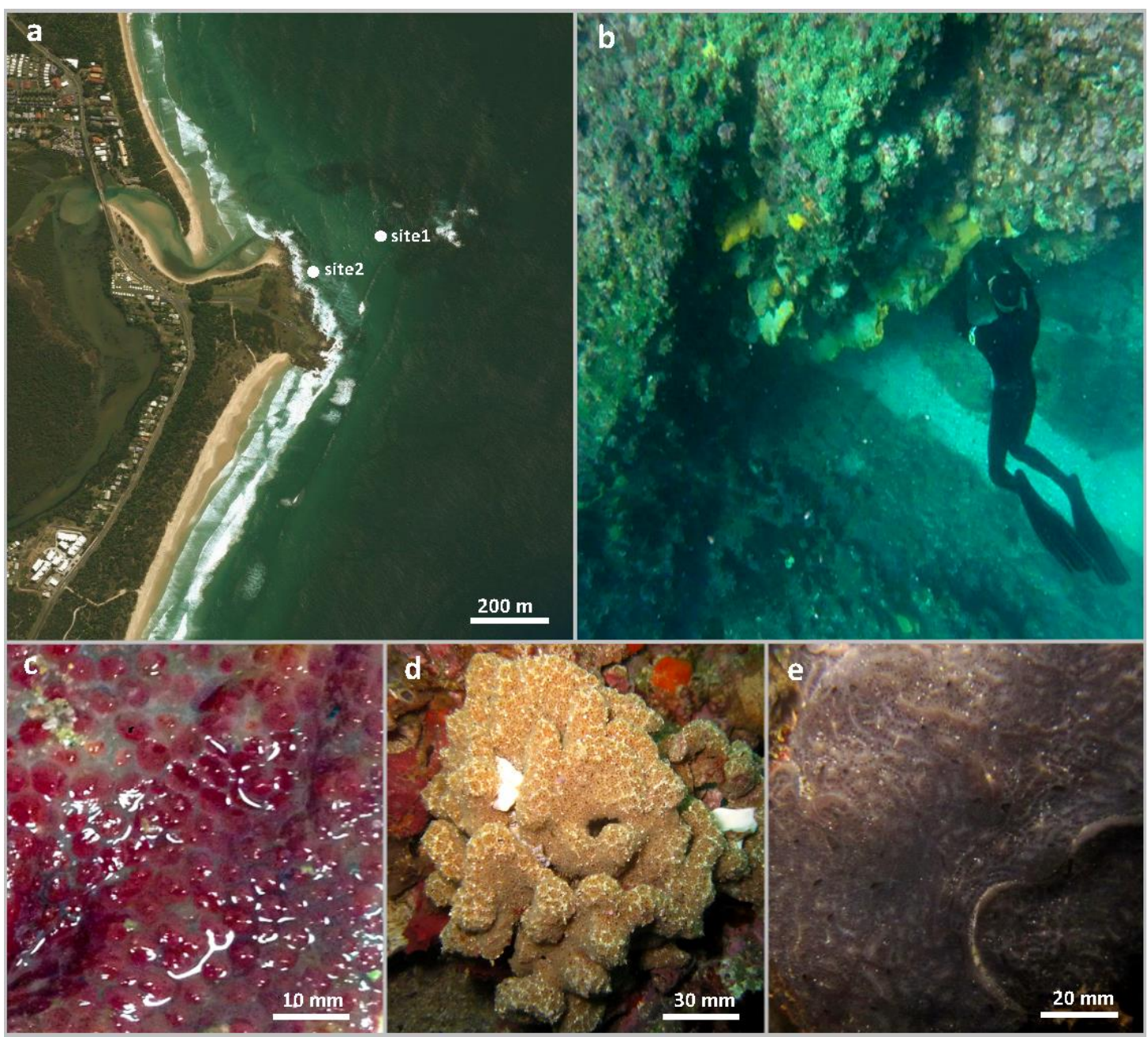

Figure 1. The Northern New South Wales coastline and in-situ aspects of the Hastings Point rock pools (a) Location of Hastings Point along the Northern New South Wales coastline and sampling sites; ${ }^{32}$ (b) Topography of the rocky reef at site 1 ; (c) $S$. rubra; (d) A. solidium; (e) P. vasculosum. 


\subsubsection{Culture-independent microbial diversity analysis}

Three $1 \mathrm{~mm}^{3}$ cubes were cut out from different locations of each ascidian specimen to allow a representative sample of the whole organism. The tissue sections were washed with sterile seawater and microbial DNA was extracted with the FastDNA SPIN Kit for Soil (MP Biomedicals). The extracted DNA was sent to the Australian Genome Research Facility (AGRF) for microbial diversity Illumina sequencing. The DNA samples were amplified using the primers 341F (CCTAYGGGRBGCASCAG) and 806R (GGACTACNNGGGTATCTAAT) for the V3 and V4 regions of the 16S rRNA gene. Forward and reverse microbiome sequences were quality filtered with Trimmomatic and joined using PandaSeq ${ }^{33,34}$ In the software package Quantitative Insights Into Microbial Ecology (QIIME) version 1.9.1, the joined sequences of all three ascidian microbiomes were combined to one fasta file using the command add_qiime_labels.py. ${ }^{35}$ The workflow script pick_de_novo_otus.py was used to pick de novo OTUs (Operational Taxonomic Units) based on 97\% sequence similarity; one representative sequence for each OTU was selected and used to assign taxonomy; subsequently, OTU sequences were aligned to build a phylogenetic tree. The summarize_taxa_through_plots.py script was run to group OTUs to phylum, class and family levels; these tables were exported to Microsoft Excel to illustrate the phylum assignments as a bar chart. Alpha rarefaction plots were generated through alpha_rare_faction.py. The sequences of OTUs belonging to actinomycetes were individually searched against the BLASTn database to confirm family level assignments.

\subsubsection{Culture-dependent actinomycete isolation and identification}

The ascidian specimens were first subjected to surface sterilization by rinsing five times with sterile seawater to remove transient and loosely attached bacteria. Each ascidian was cut into small pieces of $5 \mathrm{~mm}^{3}$ that were briefly rinsed with $70 \% \mathrm{EtOH}$ and then again rinsed with sterile seawater. The ascidian tissue was homogenised with a sterile mortar and pestle with 10 $\mathrm{mL}$ of sterile seawater; the extract and ascidian tissue were transferred to a $15 \mathrm{~mL}$ Eppendorf tube and vortexed for 10 minutes. The supernatants were then diluted (1:2 and 1:20) and $250 \mu \mathrm{L}$ were plated in duplicates on agar plates. Five different microbiological media; Difco marine agar 2216 (Difco Laboratories, USA), Starch Casein Agar (SCA; 10.0 g starch, 2.0 g K$_{2} \mathrm{HPO}_{4}$, $2.0 \mathrm{~g} \mathrm{HNO}_{3}, 2.0 \mathrm{~g} \mathrm{NaCl}, 0.3 \mathrm{~g}$ casein, $0.05 \mathrm{~g} \mathrm{MgSO}_{4} \cdot 7 \mathrm{H}_{2} \mathrm{O}, 20.0 \mathrm{~g}$ agar), ${ }^{36}$ Starch Agar (SA; $10.0 \mathrm{~g}$ glucose, $10.0 \mathrm{~g}$ potato starch, $10.0 \mathrm{~g}$ corn starch, $3.0 \mathrm{~g}$ yeast extract, $4.0 \mathrm{~g}$ peptone, $4.0 \mathrm{~g}$ beef extract, $33.3 \mathrm{~g}$ artificial sea salt, $20.0 \mathrm{~g}$ agar), ${ }^{37}$ Corn Starch (CS; $20.0 \mathrm{~g}$ corn starch, $10.0 \mathrm{~g}$ glucose, $10.0 \mathrm{~g}$ peptone, $6.0 \mathrm{~g}$ yeast extract, agar $20.0 \mathrm{~g}){ }^{37}$ Nocardia histidans medium (NH; $10.0 \mathrm{~g}$ yeast extract, $10.0 \mathrm{~g}$ glucose, $0.95 \mathrm{~g} \mathrm{Na}_{2} \mathrm{HPO}_{4}, 0.91 \mathrm{~g} \mathrm{KH}_{2} \mathrm{PO}_{4}, 0.5 \mathrm{~g} \mathrm{MgSO}_{4} \cdot 7 \mathrm{H}_{2} \mathrm{O}, 20.0$ $\mathrm{g}$ agar); ${ }^{38}$ were used for isolation of actinomycetes. All media were supplemented with 50 $\mu \mathrm{g} / \mathrm{mL}$ cycloheximide and $50 \mu \mathrm{g} / \mathrm{mL}$ nystatin to prevent fungal growth as well as $25 \mu \mathrm{g} / \mathrm{mL}$ 
nalidixic acid to inhibit the growth of background non-actinomycete bacteria. After 8 days of incubation at $28{ }^{\circ} \mathrm{C}$, actinomycete colonies were picked from the plates and re-streaked on fresh Oatmeal Agar plates (OMA; $20 \mathrm{~g}$ oatmeal, $20 \mathrm{~g}$ bacteriological agar, $3 \mathrm{~g}$ yeast extract $)^{39}$ for purification and morphological investigation; this was repeated until pure cultures were obtained. For long term storage actinomycete isolates were kept in $20 \%$ glycerol at $-20{ }^{\circ} \mathrm{C}$ and $-80{ }^{\circ} \mathrm{C} .^{40}$

\subsubsection{Molecular identification and phylogenetic analysis of actinomycete isolates}

Actinomycete DNA was extracted from individual colonies using the DNeasy Blood and Tissue Kit (Qiagen, Germany) per the manufacturer's instructions for gram-positive microorganisms. PCR was performed to amplify the $16 \mathrm{~S}$ rRNA gene, therefore, $4 \mu \mathrm{L}$ of DNA extract were used as template in $40 \mu \mathrm{L}$ solution containing $1 \mathrm{mM}$ of forward primer $27 \mathrm{~F}$ (AGAGTTTGATCMTGGCTCAG) and $1 \mathrm{mM}$ of reverse 1492R (TACGGYTACC TTGTTACGACTT), $20 \mu \mathrm{L}$ of TopTaq Master Mix (Qiagen, Germany), and $14 \mu \mathrm{L}$ of sterile $\mathrm{H}_{2} \mathrm{O}$. Amplification was performed on a Thermocycler 100 (BioRad, USA) under following conditions: initial denaturation ( $10 \mathrm{~min}$ at $\left.95^{\circ} \mathrm{C}\right)$; followed by 25 cycles of denaturation $(10 \mathrm{sec}$ at $95{ }^{\circ} \mathrm{C}$ ), primer annealing $\left(15 \mathrm{sec}\right.$ at $\left.55^{\circ} \mathrm{C}\right)$, primer extension $\left(1: 20 \mathrm{~min}\right.$ at $\left.72{ }^{\circ} \mathrm{C}\right)$; and final extension $\left(10 \mathrm{~min}\right.$ at $\left.72{ }^{\circ} \mathrm{C}\right)$. To test whether the amplification was successful, gel electrophoresis was conducted at $100 \mathrm{~V}$ for 45 minutes on $1.2 \%$ agarose gel in $0.6 \%$ TAE buffer stained with ethidium bromide. The presence of the 16S rRNA fragment was inspected under UV light using a Bio-Rad ChemiDoc XRS+ imaging system. Sequencing was performed by Macrogen Inc., Korea (http://macrogen.com/eng/). Actinomycete DNA sequences were pairwise aligned and trimmed in Geneious R6.1.8; the data was then searched against the National Center for Biotechnology Information (NCBI) nucleotide database (http://www.ncbi.nlm.nih.gov/BLAST/) to find the closest relative based on \%-identity and query cover scores. All associate sequences were multiple aligned using MUSCLE (MUltiple Sequence Comparison by Log-Expectation). ${ }^{41}$ The best evolutionary model was determined based on the lowest BIC (Bayesian Information Criterion) and AIC (Akaike Information Criterion) values. A Maximum Likelihood phylogeny was constructed in MEGA 7.0.21 using the model GTR $+\mathrm{I}+\mathrm{G}$. The $16 \mathrm{~S}$ rRNA sequence for Bifidobacterium angulatum was downloaded from NCBI and used as an outgroup. The robustness of the phylogenetic tree was evaluated by bootstrap analysis with 1000 re-samplings.Furthermore, the sequence data was imported into QIIME, de novo OTUs were established using pick_de_novo_otus.py based on 99.9\% sequence similarity. 


\subsubsection{Metabolite diversity}

The 120 actinomycete isolates were incubated in Corning ${ }^{\circledR}$ MiniBioreactors with $30 \mathrm{~mL}$ ASW-A (20.0 g soluble starch, $10.0 \mathrm{~g}$ glucose, $5.0 \mathrm{~g}$ peptone, $5.0 \mathrm{~g}$ yeast extract, $5.0 \mathrm{~g} \mathrm{CaCO}_{3}$ per litre of artificial seawater) at $28{ }^{\circ} \mathrm{C}$ and $250 \mathrm{rpm}$. After 2 weeks, the tubes were centrifuged at $8000 \mathrm{rpm}$ for 10 minutes, and the liquid media supernatant was collected. Cells were washed with $15 \mathrm{~mL}$ chilled deionised water, vortexed and centrifuged at $8000 \mathrm{rpm}$ and again supernatant was collected. Methanol $(15 \mathrm{~mL})$ was added to the harvested microbial cell pellet and the mixture was incubated on a rotational shaker for $2 \mathrm{~h}$ at room temperature and $200 \mathrm{rpm}$. The liquid phase was separated by centrifugation $(8000 \mathrm{rpm}, 5 \mathrm{~min})$ and subsequently collected. The methanol extraction of the cells was repeated with rotation overnight. The combined extracts were filtered using a $0.22 \mu \mathrm{m}$-pore-size filter and dried under pressure (GeneVac Technologies HP-12). Methanol was chosen as the extraction solvent due to its amphiphilic characteristics that enable extraction of a very broad range of polar and non-polar metabolites.

Microbial and ascidian crude extracts were prepared at $2 \mathrm{mg} / \mathrm{mL}$ and analysed on a 6530 Accurate Mass Q-TOF spectrometer (Agilent Technologies) workstation coupled to an Agilent 1260 LC system using positive electrospray ionization. A Kinetex ${ }^{\circledR} \mathrm{C}_{18} 5 \mu \mathrm{m} 100 \AA$ HPLC column $(100 \times 4.6 \mathrm{~mm})$ was used under chromatographic conditions from 0 - $100 \% \mathrm{ACN} /$ $0.1 \%$ FA over $20 \mathrm{~min}$. The Q-TOF MS was set to positive mode mass range $\mathrm{m} / \mathrm{z} 100-1700$, MS scan rate 3 spectra/sec, MS/MS scan rate 2/sec, fixed collision energies at 10, 20 and $40 \mathrm{keV}$. Source parameters were set to gas temperature $300^{\circ} \mathrm{C}$, gas flow $10 \mathrm{~L} / \mathrm{min}$, nebulizer 35 psig; and scan source parameters were: VCap 3500, nozzle voltage $1500 \mathrm{~V}$, fragmentor $130 \mathrm{~V}$, skimmer $65 \mathrm{~V}$, and octopoleRFPeak $750 \mathrm{~V}$.

Every fifth acquired sample was followed by a blank. At the beginning and end of the LCMS/MS acquisition, a $10 \mu \mathrm{g} / \mathrm{mL}$ mixed pesticide standard (acidic and basic, Agilent Technologies) was tested as a quality control. Data was inspected for integrity in MassHunter software (Agilent Technologies). For data analysis, all MS/MS data was converted from Agilent MassHunter data files to mzXML file format using MSConvert. The data was uploaded to the GNPS server (gnps.ucsd.edu). A molecular network was created using the online workflow at GNPS. The data was filtered by removing all MS/MS peaks within +/- $17 \mathrm{Da}$ of the precursor $\mathrm{m} / \mathrm{z}$. MS/MS spectra were window filtered by choosing only the top 6 peaks in the $+/-50 \mathrm{Da}$ window throughout the spectrum. The data was then clustered with MS-Cluster with a parent mass tolerance of 2.0 Da and a MS/MS fragment ion tolerance of $0.5 \mathrm{Da}$ to create consensus spectra. Consensus spectra that contained less than 2 spectra were discarded. A network was then created where edges were filtered to have a cosine score above 0.7 and more than 4 matched peaks. Further edges between two nodes were kept in the network only if each of the 
nodes appeared in each other's respective top 10 most similar nodes. The spectra in the network were then searched against GNPS spectral libraries. The library spectra were filtered in the same manner as the input data. All matches kept between network spectra and library spectra were required to have a score above 0.7 and at least 4 matched peaks. ${ }^{42}$ The molecular network was visualised in Cytoscape 3.3.0., where all mass ions associated with the blanks and mixed pesticide standard were removed from the network. Nodes were coloured per actinomycete taxa group or ascidian group. The network figure was edited in Inkscape 0.92 freeware.

Euler-Venn diagrams were generated with eulerAPE v3 (http:// www.eulerdiagrams.org/ eulerAPE) to depict the numbers of unique and shared actinomycete OTUs between the three ascidians based on the data obtained through culture-independent and culture-dependent approaches. Similarly, shared secondary metabolite mass ions of the three ascidians as well as their cultured ascidian-associated actinomycetes were analysed. Thereby, any primary metabolites that were identified against the GNPS database were excluded from the Euler-Venn diagrams.

\subsection{Results}

\subsubsection{Microbial diversity associated with three Australian ascidians}

We evaluated the microbial diversity of Australian ascidians using a culture-independent approach targeting ascidian genera, whose microbiomes had not previously been studied in breadth. The samples were collected at an intertidal habitat at Hastings Point, New South Wales, Australia and based on morphological traits the specimens were taxonomically assigned to Symplegma rubra (Figure 1c), Aplidium solidum (Figure 1d) and Polyclinum vasculosum (Figure 1e).

16S rRNA gene Illumina sequencing of the V3 - V4 region resulted in a total of 287758 sequences for the three ascidians. After quality filtering, 253962 sequences remained for analysis and 13945 OTUs were determined based on 97\% sequence similarity. Species richness was established through alpha rarefaction and compared between the three ascidian samples. From the rarefaction curves (Figure 2a) it was apparent that S. rubra, which was the only sample collected at the small rocky reef approximately 100 meters offshore, had a more diverse microbial community than the other two ascidian species, $P$. vasculosum and A. solidum, which were obtained from sheltered intertidal rock pools. 
Nineteen different bacterial phyla were observed and all three ascidian samples were dominated by Proteobacteria (54 - 58\%), followed by Cyanobacteria, which showed a larger variation between $11-35 \%$. All ascidians contained a fraction of unassigned phyla; specifically, in $P$. vasculosum this fraction had a high contribution of $28 \%$ of the overall bacterial diversity. The phylum Actinobacteria was present in all three ascidians; however, in a far smaller portion, with the highest percent observed in S. rubra being $1.91 \%$, while A. solidum and P. vasculosum both had values below 1\% (Figure 2b). Within the phylum Actinobacteria, the order Actinomycetales is of particular interest due to their ability to produce many bioactive metabolites. Therefore, we further inspected the order Actinomycetales to the family level (Table 1). Sequences of the taxonomic families Corynebacteriaceae, Microbacteriaceae, Nocaridaceae and Sterptomycetaceae were detected in all three ascidians, yet at the OTU level (97\%) only two OTUs were shared between all three ascidians (Table 1 and Figure 3a). Thermomonosporaceae and Mycobacteriaceae had the highest number of OTUs, but sequences were only detected in S. rubra and P. vasculosum. In these two ascidians, the natural product producing families Micromonosporaceae and Streptosporangiaceae were discovered, as well as Kineosporiaceae. A. solidum exhibited the lowest diversity of actinomycete families; while Actinomycetaceae and Micrococcaceae were unique to this ascidian, Pseudonocardiaceae OTUs were shared with $S$. rubra. Four OTUs that could not be identified to family level unambiguously were obtained from A. solidum and $P$. vasculosum. All other observed actinomycetes family OTUs were unique to only one ascidian (Table 1).
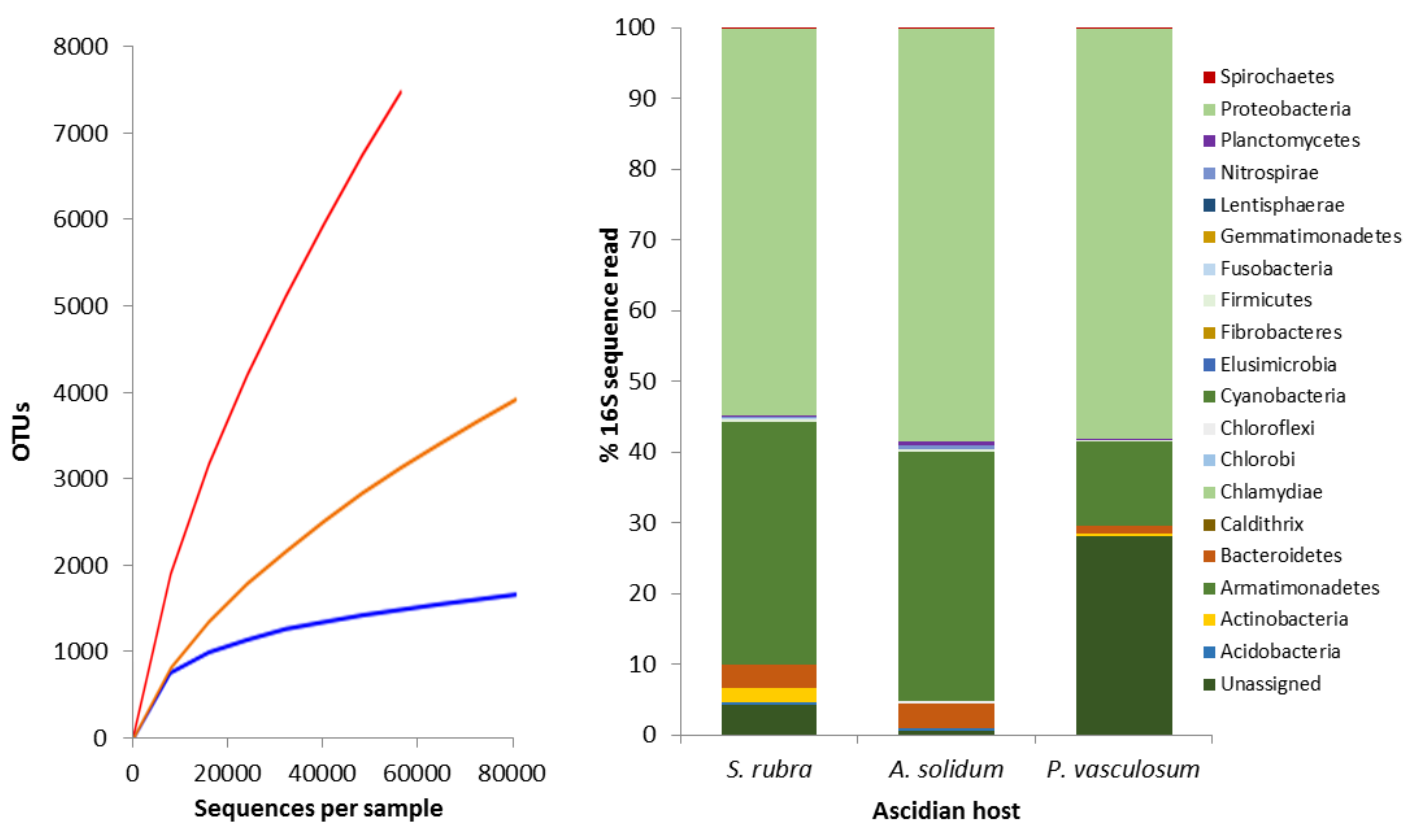

Figure 2. Microbial diversity analysis (a) Average alpha rarefaction curve showing that $S$. rubra contained the highest microbial diversity, while $A$. solidium had the least diverse microbial community; (b) Percent diversity of taxonomic phyla assigned to three Australian ascidian species. 
Table 1. Summary of de novo OTUs representing the taxonomic order "Actinomycetales".

\begin{tabular}{ccc}
\hline $\begin{array}{c}\text { No of } \\
\text { OTUs }\end{array}$ & Family & Ascidian host \\
\hline 1 & Acidothermaceae & Polyclinum vasculosum \\
1 & Actinomycetaceae & Aplidium solidium \\
1 & Cellulomonadaceae & Polyclinum vasculosum \\
2 & Corynebacteriaceae & Symplegma rubra, Polyclinum vasculosum, Aplidium solidium \\
1 & Gordoniaceae & Symplegma rubra \\
2 & Intrasporangiaceae & Polyclinum vasculosum \\
1 & Kineosporiaceae & Symplegma rubra, Polyclinum vasculosum \\
5 & Microbacteriaceae & Symplegma rubra, Polyclinum vasculosum, Aplidium solidium \\
1 & Micrococcaceae & Aplidium solidium \\
3 & Micromonosporaceae & Symplegma rubra, Polyclinum vasculosum \\
6 & Mycobacteriaceae & Symplegma rubra, Polyclinum vasculosum \\
5 & Nocardiaceae & Symplegma rubra, Polyclinum vasculosum, Aplidium solidium \\
1 & Promicromonosporaceae & Polyclinum vasculosum \\
1 & Pseudonocardiaceae & Symplegma rubra Aplidium solidium \\
4 & Streptomycetaceae & Symplegma rubra, Polyclinum vasculosum, Aplidium solidium \\
3 & Streptosporangiaceae & Symplegma rubra, Polyclinum vasculosum \\
6 & Thermomonosporaceae & Symplegma rubra, Polyclinum vasculosum \\
4 & Ambiguous assignment & Symplegma rubra, Polyclinum vasculosum \\
\hline
\end{tabular}

a

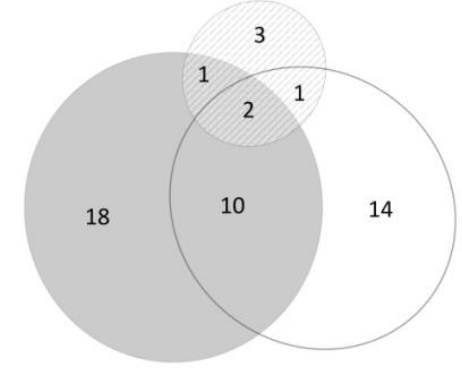

Detectable ascidian-associate actinomycete OTUs

C

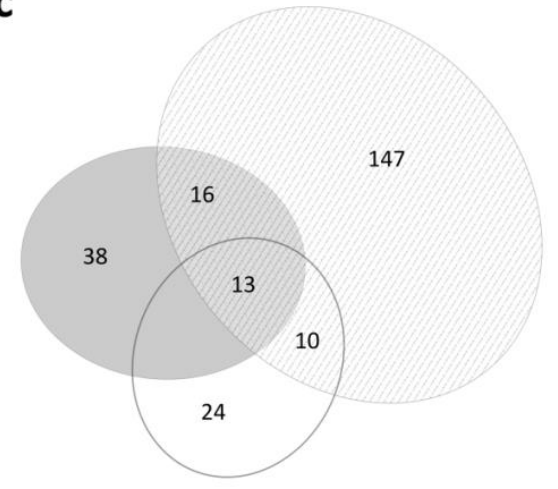

Ascidian metabolites b

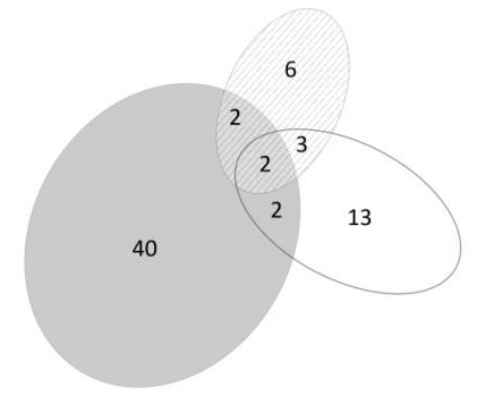

Culturable ascidian-associate actinomycete OTUs

d

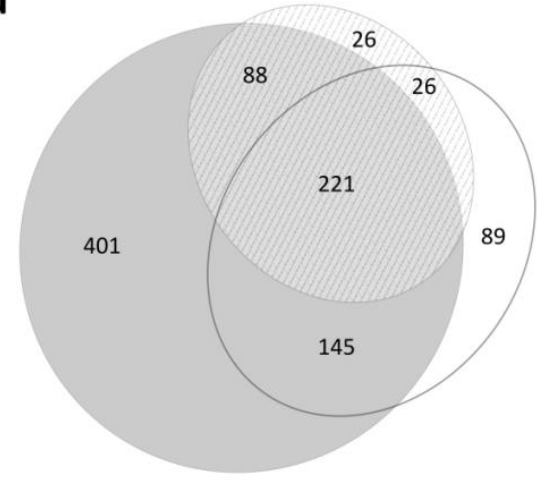

Ascidian-associate actinomycete metabolites

Symplegma rubra

Figure 3. Microbial and secondary metabolite overlaps between the three Australian ascidians in terms of (a) detectable actinomycete OTUs (b) culturable actinomycete OTUs (c) molecular ions (d) associate metabolites. (Note: primary metabolite database hits were excluded from this diagram). 


\subsubsection{Culture-dependent actinomycete diversity}

Once the presence of actinomycete sequences in the different ascidians was confirmed through culture-independent techniques, traditional culture-dependent microbiological techniques were used to obtain the culturable actinomycete diversity. This resulted in the isolation of 120 diverse actinomycete-like isolates. Phylogenetic analysis was used to confirm their taxonomy. Therefore, the 16S rRNA gene was amplified for all culturable actinomycetelike isolates and subsequently sequenced. All sequences were amplified successfully for more than $1200 \mathrm{bp}$ using the $27 \mathrm{~F}$ and $1492 \mathrm{R}$ universal primers. The 120 sequences were searched against the NCBI 16S ribosomal RNA database for bacteria and archaea and sequences of their closest phylogenetic relative were obtained from the database and included in the phylogenetic analysis. One hundred and eighteen strains exhibited $99 \%$ and above similarities to previously described actinomycete strains. Lower sequence similarities were observed for Streptomyces isolates USC-16002 (98\%) and USC-16062 (97\%), both isolated from the S. rubra, suggesting that these are potential candidates of novel actinomycete taxa.

The maximum likelihood analysis was used to estimate the phylogenetic relationship of the ascidian-associated actinomycete isolates and revealed four major clades with high statistical support (bootstrap values of 98-100\%). This included a clade for Micromonospaceae, Streptosporangaceae, Streptomyceae and a Nocardiacea clade that further branched into wellsupported Nocardia and Rhodococcus genus clades (Figure 4).

The three ascidian specimens yielded a total of 120 actinomycete isolates originating from five different genera: Micromonospora (58\%), Streptomyces (34\%), Nocardia (5\%), Rhodococcus (2\%) and Streptosporangium (1\%) (Table S1). Four actinomycete-specific isolation media and one generic commercially available marine agar were chosen for isolation. The selection of media had a notable influence on the number of isolates recovered. Starch Casein Agar (SCA) allowed the highest recovery of isolates and a total of 60 actinomycetes were obtained, predominantly from the genus Micromonospora. The highest actinomycete diversity was recovered from Corn Starch (CS) media; however, it appeared most selective for Streptomyces species. Starch Agar (SA) was highly selective for Micromonospora species and yielded 20 Micromonospora isolates and only one Streptomyces species. NH media only recovered one Nocardia sp. isolate. A low number of actinomycetes (6 isolates) were obtained from Marine Agar (MA); but interestingly, the only Streptosporangium isolate (USC-16119) was recovered from this media (Figure 5a). 


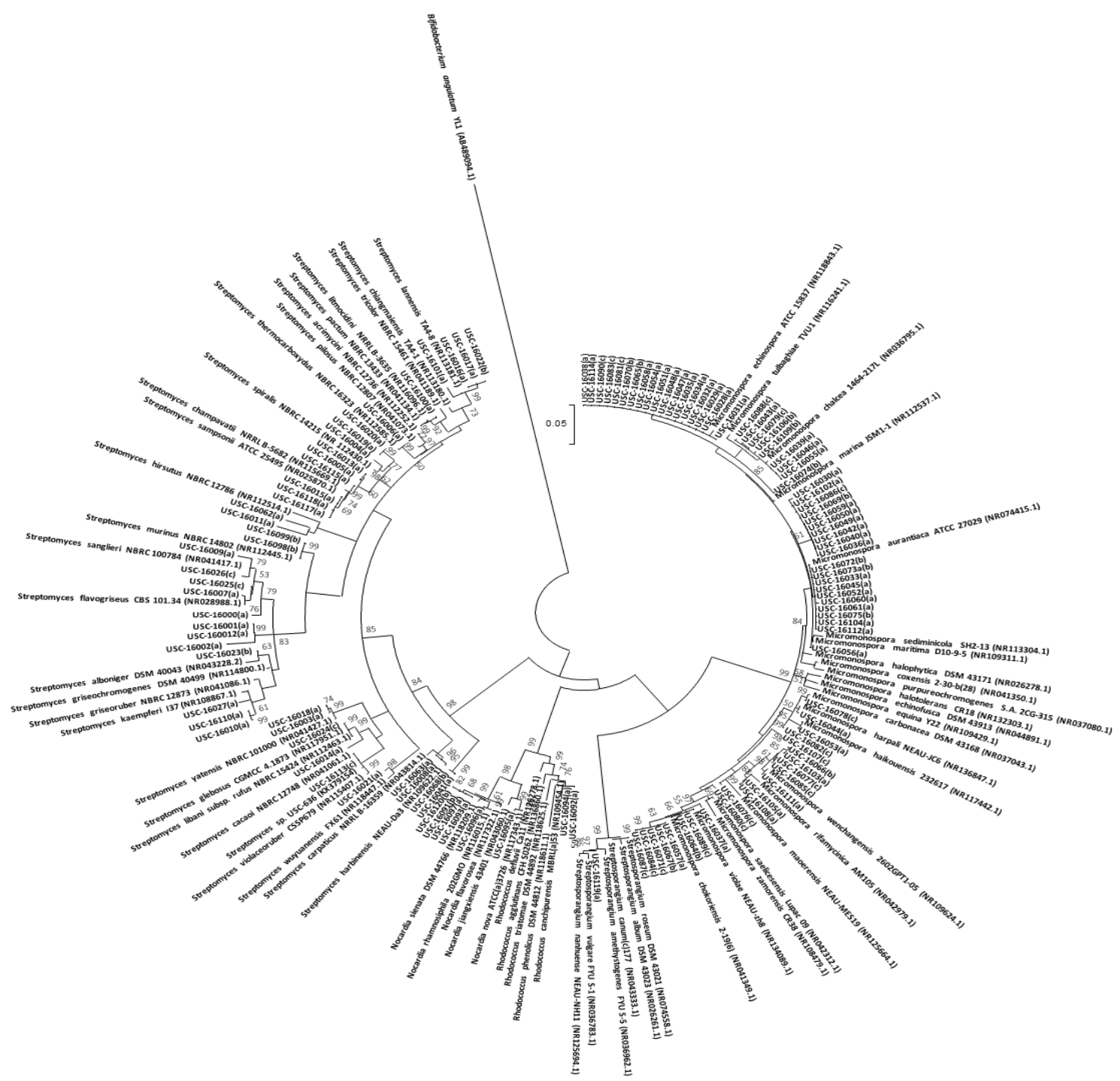

Figure 4. Phylogenetic analysis of the actinomycete isolates in relation to their closest relatives. (Bifidobacterium angulatum (AB489094) was used as an out-group sequence to root the tree) Isolates obtained from $S$. rubra are indicated through (a), A. solidium isolates (b) and $P$. vasculosum (c).

The recovery of actinomycetes varied between the three ascidian samples. S. rubra yielded the highest number (80) and diversity of isolates with representatives of all five isolated actinomycete genera, Micromonospora, Streptomyces, Nocardia, Rhodococcus and Streptosporangium (Figure 5b). A. solidum and $P$. vasculosum, which were both collected from the shore rock pools, yielded a similar number of isolates, 19 and 21 respectively, but much lower numbers and diversity in comparison to $S$. rubra, which was collected from the rocky reef $100 \mathrm{~m}$ offshore (Figure 1a). The isolation success, therefore, reflects the observed actinomycete distribution detected through molecular analysis 


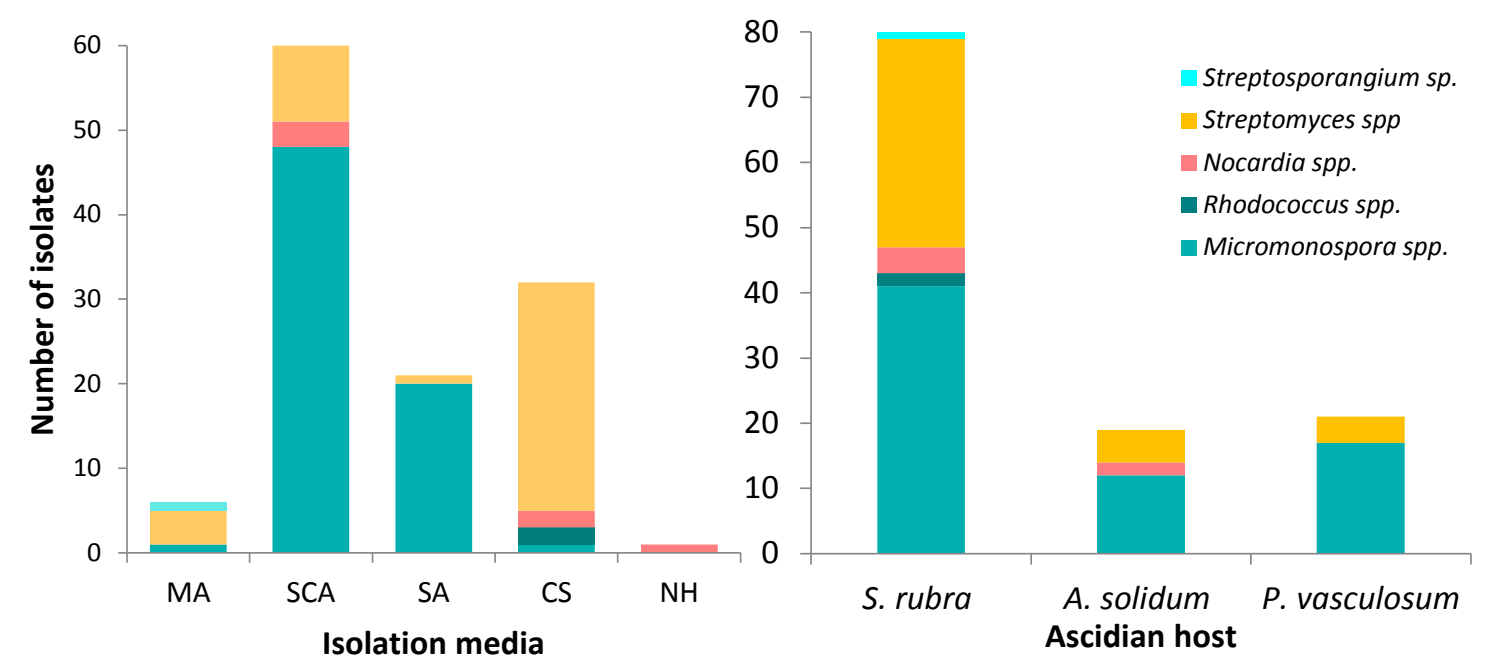

Figure 5. Numbers and taxonomic diversity of actinomycetes (a) isolated from five different isolation media; (b) derived from three Australian Ascidians.

As indicated in the phylogenetic tree, several of the isolated actinomycetes appeared to be identical; therefore, the QIIME toolbox was utilised to determine culturable actinomycete OTUs, which were compared between three ascidian samples. The 120 actinomycete sequences clustered into a total of 78 OTUs based on $99.9 \%$ sequence similarity (Appendix I, Table IIA). Figure 3 depicts the microbial and metabolite overlaps between the three Australian ascidians. Interestingly, only two microbial OTUs were shared between all tree ascidian specimens. This suggests that the isolated actinomycete associates were mostly unique to their ascidian host. Specifically, the Streptomyces, except for one OTU (comprised for one isolate from S. rubra and one from A. solidum), showed no overlap between ascidian samples (Table S1). The numbers of detectable and culturable actinomycetes OTUs shared between the ascidians appeared mostly proportional, only shared OTUs between S. rubra and P. vasculosum were higher with ten shared OTUs compared to the two cultured OTUs (Figure 3a/b). Interestingly, the two actinomycete OTUs shared between all three ascidians that were defined based on the culture-independent methods belonged to the families of Streptomycetaceae and Nocardiaceae. We compared the sequences from culture-independent and dependent approaches and found that they well aligned, but none of the sequences matched $100 \%$. However, 16S sequence fragments in the culture independent analysis were maximal 465 bp long and of lower quality, while sequences of $1200 \mathrm{bp}$ were recovered from the cultured isolates, making definite conclusions about shared OTUs difficult. 


\subsubsection{Metabolite diversity}

LC-MS/MS data was collected for the three ascidian hosts as well as extracts of the actinomycete associates. In contrast to the microbial diversity, A. solidum had the highest secondary metabolite diversity (186 molecular ions) followed by $S$. rubra (73 ions) and $P$. vasculosum had the lowest ion diversity (55 ions). Thirteen molecular ions were found in all three ascidian specimens (Figure 3c). The metabolite diversity of the ascidian-associated actinomycetes reflected the diversity of the microbial diversity and $S$. rubra-associated actinomycetes by far had the highest abundance of molecular ions (Figure 3d). For visualisation, a molecular ion network was constructed (Figure 6). After removal of ions present in blanks and standards, a total of 1532 parent ions were observed in the samples and represented as nodes coloured by taxa where edges indicate significant pairwise alignment between nodes. The network shows several taxa-specific node clusters; specifically, Streptomyces and Micromonospora display a diverse plethora of unique molecular ion clusters. With 842 detected ions, Streptomyces exhibited the richest metabolite diversity followed by 681 ions detected in Micromonospora isolates. Nocardia showed several unique clusters but had most ion overlap with Micromonospora. Only one Streptosporangium isolate was included in the analysis, yet its metabolite diversity is noteworthy with a total of 91 molecular ions detected of which 20 were unique and 73 coincided with the Micromonospora ions. Seventy-four metabolites could be identified through MS/MS spectra matches against the GNPS database (Table S2). The majority of the database matches present common primary metabolites, such as nucleotides, sugars, amino acids, fatty acids, vitamins and cell membrane components (phosphatidylcholines), which are of less interest for biotechnological or pharmaceutical studies. Furthermore, several linear and cyclic dipeptides, as well as other previously described bioactive secondary metabolites were identified (Figure 6a). Surugamide A and leupeptin, both protease inhibitors previously isolated from Streptomyces sp., ${ }^{43,44}$ which could be annotated to Streptomyces specific clusters (Figure $\mathbf{6 b} / \mathbf{c}$ ). Rakicidin A and B were attributed to a small cluster of only three nodes shared between several Micromonospora and the Streptosporangium isolates (Figure 6d); these compounds were previously reported to exhibit cytotoxic and antitumor properties. ${ }^{45}$ Additionally, the common actinomycete siderophore for iron acquisition, desferrioxamine B, was linked to a shared actinomycete cluster (Figure 6e). The remaining great majority of ions in the networks could not be assigned against the GNPS database. 


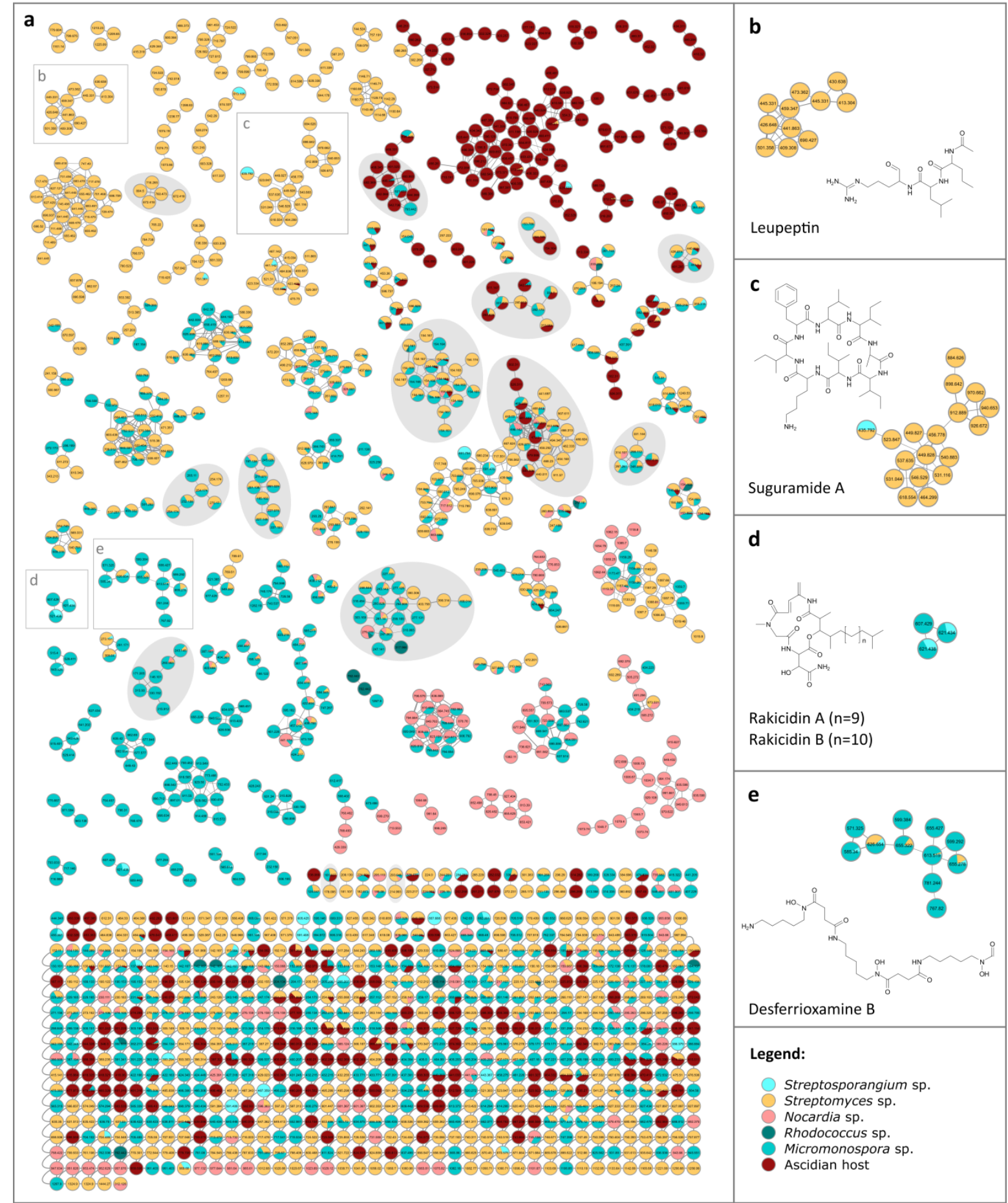

Figure 6. Ascidian-associated actinomycete molecular network: (a) overall molecular network, where node colour represents different actinomycete taxa and ascidian hosts; networks with identified primary metabolites are highlighted with grey ellipses. Identified secondary metabolite networks of (b) leupeptin; (c) surugamide A; (d) rakicidin A and B; (e) desferrioxamine $B$. 
Of great interest were the nodes that overlapped between ascidian hosts and their microbial associates, as these nodes are likely to be compounds that are produced by a microbial associate of the ascidians. Only one detected ion with a molecular mass of 479.9 Da was identified as an oxobutanoic acid derivative and was found in all three ascidians and Micromonospora and Nocardia associates. Ninety-one molecular ions coincided between $S$. rubra and its actinomycete associates. Whilst $P$. vasulosum had 74 overlapping ions, and in A. solidum 52 ions matched the associates. Micromonospora and Streptomyces isolate metabolomes showed the greatest overlap and thus appear to contribute to the ascidian metabolome. Of great interest were the nodes that overlapped between ascidian hosts and their microbial associates, as these nodes are likely to be compounds that are produced by a microbial associate of the ascidians. Only one detected ion with a molecular mass of 479.9 Da was identified as an oxobutanoic acid derivative and was found in all three ascidians and Micromonospora and Nocardia associates. Ninety-one molecular ions coincided between S. rubra and its actinomycete associates. Whilst P. vasulosum had 74 overlapping ions, and in A. solidium 52 ions matched the associates. Micromonospora and Streptomyces isolate metabolomes showed the greatest overlap and thus appear to contribute to the ascidian metabolome. Rhodoccocus had only 8 ions that also were found in ascidian hosts, suggesting that Rhodoccocus may have been acquired by chance from the environment and probably does not play a role in these ascidians.

\subsection{Discussion}

In this study, we compared the microbiomes and metabolomes of three Australian ascidian species collected from the same geographical location at Hastings Point, NSW, Australia. To date, scientists still rely on culture-dependent methodologies in order to study the expressed secondary metabolome and its biological activity; however, this approach might miss out on unculturable microbial strains. Therefore, we initially determined the microbial diversity associated with the three ascidians based on a culture-independent approach and then compared the findings with the culturable actinomycete diversity. We further intended to provide insight into the functional role of actinomycetes in associations with ascidians and connect the results to the production of bioactive secondary metabolites.

At the phylum level, the overall microbial diversities and distributions associated with the three Australian ascidian specimens were comparably similar. Other culture-independent ascidian microbiome studies from different geographical regions, including Asian-Pacific, South Pacific, and the Caribbean reported similar phylum distributions, where Proteobacteria were generally reported as the most dominant phylum and also Cyanobacteria were commonly found in high abundance. ${ }^{9,17,19,46-48}$ Actinobacteria were usually reported in lower numbers in 
these studies. However, low numbers of actinobacterial sequences could potentially be attributed to poor DNA extraction and primer biases of the V3 - V4 region of the 16S rRNA gene. ${ }^{49}$ Particularly, for Gram-positive bacteria, DNA extractions have been proven difficult and most commercial DNA extraction kits require specific cell lysis pre-treatments of the samples, which could be a reason that higher numbers of Gram-negative bacteria were reported. ${ }^{49}$

The isolation media were targeted at mycelium forming actinomycete genera, this included Streptomyces, Micromonospora, and Nocardia, which are renowned to produce bioactive secondary metabolites. As detected in the culture-independent sequencing analysis, we successfully isolated highly diverse Streptomyces species strains from all ascidian samples. Micromonospora species were also obtained in high diversity and abundance from all three ascidians, while only S. rubra and P. vasculosum showed Micromonosporaceae OTUs in the culture-independent analysis. Other actinomycete resembling family members, including Gordoniaceae, Corynebacteriaceae, and Micrococcaceae colonies were also observed on isolation plates, confirming culture-independent data; however, due to time constraints were not targeted for isolation. Streptosporangiaceae sequences were identified in $S$. rubra and $P$. vasculosum, but could only be recovered from S. rubra, which could have been affected by the choice of isolation media. Nocardia and Rhodococcus were isolated in very low numbers and displayed little diversity that might be due to their transitory characteristics within the ascidian hosts. Similarly, the chosen isolation media are commonly used for a broad range of actinomycete taxa and may not have been selective enough to culture these genera.

It has been proposed that microbial diversity of ascidians is stable and species-specific, but it also has a location-specific aspect where microorganisms are acquired from the local environment. ${ }^{9,10}$ Actinomycetes are commonly found in the marine as well as the terrestrial environment. In fact, it is commonly believed that the order is of terrestrial origin and its spores have been introduced to the marine environment as wash off and then adapted to the saline conditions. ${ }^{50-53}$ Hence, it is likely that actinomycetes are facultative symbionts, meaning that they are acquired from the environment and thus should be location-specific rather than host specific. ${ }^{10,54}$ Nonetheless, actinomycetes still present a valuable target species for biodiscovery, especially as it is proposed that as they adapt to the marine environment this adaptation also leads to a change in their expressed secondary metabolome. ${ }^{50,53,55-56}$ If actinomycete associations are facultative, then actinomycete diversity of ascidians sampled at the same location and time should greatly overlap. The study on these three different ascidians collected at nearby geographic locations showed otherwise; most culturable actinomycete isolates were unique to only one ascidian. Only two cultured actinomycete OTUs were shared between the three different ascidian species; it is noteworthy that these strains were fairly abundant, pointing towards a location factor. $S$. rubra, which was sampled at the small rocky reef approximately 
200 meters offshore, had a more diverse microbial community based on Illumina sequencing data as well as culturable actinomycete diversity compared to the other two ascidian species. It might be possible that $S$. rubra receives more microorganisms through oceanic currents and terrestrial waters from the river mouth of Cudgara Creek adjacent to the sampling site (Figure 1). A. solidum and P. vasculosum were obtained from rock pools just off the mainland that provide more shelter from oceanic currents. Additionally, A. solidum incorporate sand internally into their tissues as they form hard rigid colony structures; ${ }^{57}$ therefore, the low microbial diversity associated with $A$. solidum might be attributed to its tissue being significantly higher in sand content, allowing for less living tissue per standard volume. Since, the overlap of actinomycete isolates between the different ascidian species sourced from the same physiological environment was not found to be significant; these findings support the argument that the acquisition of actinomycetes is selective. It has to be taken into account that these conclusions were established based on the actinomycetes that could be derived from the chosen isolation media and solely three nearby collected specimens and further studies would be required to confirm these conclusions.

Polyclinidae after Didemnidae is the ascidian family that has yielded most reported natural products. ${ }^{30}$ Numerous secondary metabolites (138) from the genus Aplidium are listed in the Dictionary of Natural Products (http://dnp.chemnetbase.com, accessed 30-08-2017), while only three and one natural products are listed for Symplegma and Polyclinum, respectively. These reports fit the diversity of molecular ions associated with three ascidians in this study. We investigated whether metabolite diversity of the three Australian ascidians was correlated with the host's microbial diversity. Our results show that the microbial diversity did not reflect the chemical diversity. S. rubra, which contained an impressive diversity of microbial associates based on culture-independent as well as culture-dependent approaches, had lower chemical diversity by LC-MS/MS analysis than the other two ascidian specimens. While A. solidum exhibited the highest metabolite diversity, low numbers of microbial sequences and culturable actinomycetes were recovered. Similarly, Tianero et al. found no correlation between microbial and chemical diversity nor biological activity, but rather specific interactions with bioactive secondary metabolite producers. ${ }^{9}$

To gain an overview whether there were any secondary metabolites that can be attributed to actinomycete associates isolated from these ascidians we acquired LC-MS/MS profiles of all ascidians and their cultured actinomycete associates. Many nodes overlapped between ascidians and actinomycetes indicating that these compounds are potentially produced by microbial associates. Generally, ions in a molecular network are all closely related and therefore it is likely that as soon as there is one ion match between actinomycete and its host that all compounds within this network are of microbial origin. The molecular network indicates that 
actinomycetes derived from Australian ascidians contain a highly diverse metabolome that in this case even exceeds that of the ascidians and targeting actinomycete associates thus proves a valuable approach for natural product drug discovery and aids to overcome the supply problem. Even more so, as bacterial cultures can be manipulated in the laboratory, by using different fermentation media or using different chemical or physical elicitors to activate silent gene clusters in order to further induce secondary metabolite diversity. ${ }^{58}$

In this study, we presented a rich taxonomic as well as metabolite diversity of actinomycetes associated with ascidians which both appear species specific. The geographic and taxonomic scope was however limited to only three ascidian species from one habitat, therefore we regard this work as a small contribution to the bigger picture. Extended knowledge of diversity and distribution of actinomycetes in the marine environment will contribute further to a deeper understanding of their ecological function and will aid towards the design of improved biodiscovery strategies. The next steps in this work will be to identify new secondary metabolites that could not be assigned from the molecular network analysis and thus maximise the great potential of actinomycetes for natural product drug discovery.

\subsection{Conclusions}

This study has shown that the ascidians, S. rubra, A. solidum and $P$. vasculosum, harbour a range of diverse actinomycetes, which we successfully identified with culture independent methods and subsequently isolated using culture dependent techniques. The actinomycete metabolomes, specifically for Streptomyces and Micromonospora isolates, were highly diverse and thus present a good target for natural product drug discovery.

\subsection{References}

(1) Paul, V.J.; Lindquist, N.; Fenical, W. Chemical defenses of the tropical ascidian atapozoa sp. and its nudibranch predators nembrotha spp. Mar. Ecol.: Prog. Ser. 1990, 59, 109118.

(2) Lindquist, N.; Hay, M.E.; Fenical, W. Defense of ascidians and their conspicuous larvae: Adult vs. Larval chemical defenses. Ecol. Monogr. 1992, 62, 547-568.

(3) Joullié, M.M.; Leonard, M.S.; Portonovo, P.; Liang, B.; Ding, X.; La Clair, J.J. Chemical defense in ascidians of the didemnidae family. J. Bioconjugate Chem. 2003, 14, 30-37.

(4) Meyer, C.A. The global marine pharmaceuticals pipeline. http://marinepharmacology.midwestern.edu/clinPipeline.htm (04 April 2017),

(5) Schmidt, E.W.; Nelson, J.T.; Rasko, D.A.; Sudek, S.; Eisen, J.A.; Haygood, M.G.; Ravel, $\mathrm{J}$. Patellamide a and $\mathrm{c}$ biosynthesis by a microcin-like pathway in prochloron didemni, the 
cyanobacterial symbiont of lissoclinum patella. J. Proc. Natl. Acad. Sci. U. S. A. 2005, $102,7315-7320$.

(6) Schmidt, E.W. The secret to a successful relationship: Lasting chemistry between ascidians and their symbiotic bacteria. Invertebr. Biol. 2014.

(7) Leal, M.C.; Sheridan, C.; Osinga, R.; Dionísio, G.; Rocha, R.J.M.; Silva, B.; Rosa, R.; Calado, R. Marine microorganism-invertebrate assemblages: Perspectives to solve the "supply problem" in the initial steps of drug discovery. Mar. drugs 2014, 12, 3929-3952.

(8) Radjasa, O.K.; Vaske, Y.M.; Navarro, G.; Vervoort, H.C.; Tenney, K.; Linington, R.G.; Crews, P. Highlights of marine invertebrate-derived biosynthetic products: Their biomedical potential and possible production by microbial associants. Bioorg. Med. Chem. 2011, 19, 6658-6674.

(9) Tianero, M.D.B.; Kwan, J.C.; Wyche, T.P.; Presson, A.P.; Koch, M.; Barrows, L.R.; Bugni, T.S.; Schmidt, E.W. Species specificity of symbiosis and secondary metabolism in ascidians. ISME J. 2014.

(10) Erwin, P.M.; Pineda, M.C.; Webster, N.; Turon, X.; Lopez-Legentil, S. Down under the tunic: Bacterial biodiversity hotspots and widespread ammonia-oxidizing archaea in coral reef ascidians. ISME J. 2014, 8, 575-588.

(11) Jensen, P.R.; Fenical, W. Strategies for the discovery of secondary metabolites from marine bacteria: Ecological perspectives. Annu. Rev. Microbiol. 1994, 48, 559-584.

(12) Kwan, J.C.; Schmidt, E.W. Bacterial endosymbiosis in a chordate host: Long-term coevolution and conservation of secondary metabolism. PloS one 2013, 8, e80822.

(13) Kwan, J.C.; Tianero, M.D.B.; Donia, M.S.; Wyche, T.P.; Bugni, T.S.; Schmidt, E.W. Host control of symbiont natural product chemistry in cryptic populations of the tunicate lissoclinum patella. PloS one 2014, 9, e95850.

(14) Kremer, B.; Pardy, R.; Lewin, R. Carbon fixation and photosynthates of prochloron, a green alga symbiotic with an ascidian, lissoclinum patella. Phycologia 1982, 21, 258-263.

(15) Koike, I.; Suzuki, T. Nutritional diversity of symbiotic ascidians in a fijian seagrass meadow. Ecol. Res. 1996, 11, 381-386.

(16) Münchhoff, J.; Hirose, E.; Maruyama, T.; Sunairi, M.; Burns, B.P.; Neilan, B.A. Host specificity and phylogeography of the prochlorophyte prochloron sp., an obligate symbiont in didemnid ascidians. Environ. Microbiol. 2007, 9, 890-899.

(17) Donia, M.S.; Fricke, W.F.; Partensky, F.; Cox, J.; Elshahawi, S.I.; White, J.R.; Phillippy, A.M.; Schatz, M.C.; Piel, J.; Haygood, M.G. Complex microbiome underlying secondary and primary metabolism in the tunicate-prochloron symbiosis. J. Proc. Natl. Acad. Sci. U. S. A. 2011, 108, E1423-E1432.

(18) Donia, M.S.; Fricke, W.F.; Ravel, J.; Schmidt, E.W. Variation in tropical reef symbiont metagenomes defined by secondary metabolism. PLoS One 2011, 6, 17897.

(19) Steinert, G.; Taylor, M.W.; Schupp, P.J. Diversity of actinobacteria associated with the marine ascidian eudistoma toealensis. Mar. Biotechnol. 2015, 17, 377-385.

(20) Chen, L.; Fu, C.; Wang, G. Microbial diversity associated with ascidians: A review of research methods and application. Symbiosis 2016, 1-8.

(21) Jimenez, P.C.; Ferreira, E.G.; Araújo, L.A.; Guimarães, L.A.; Sousa, T.S.; Pessoa, O.D.L.; Lotufo, T.M.; Costa-Lotufo, L.V. Cytotoxicity of actinomycetes associated with the ascidian eudistoma vannamei (millar, 1977), endemic of northeastern coast of brazil/citotoxicidad de actinomicetos asociada a la ascidia eudistoma vannamei (millar, 1977), endémica de la costa noreste de brasil. Lat. Am. J. Aquat. Res. 2013, 41, 335. 
(22) Kurtböke, D.I. Ecology and habitat disctribution of actinobacteria. In Biology and Biotechnology of Actinobacteria, Springer: 2017.

(23) He, H.; Ding, W.-D.; Bernan, V.S.; Richardson, A.D.; Ireland, C.M.; Greenstein, M.; Ellestad, G.A.; Carter, G.T. Lomaiviticins a and b, potent antitumor antibiotics from micromonospora lomaivitiensis. J. Am. Chem. Soc. 2001, 123, 5362-5363.

(24) Li, A.; Piel, J. A gene cluster from a marine streptomyces encoding the biosynthesis of the aromatic spiroketal polyketide griseorhodin a. Chem. Biol. 2002, 9, 1017-1026.

(25) Hayakawa, Y.; Shirasaki, S.; Kawasaki, T.; Matsuo, Y.; Adachi, K.; Shizuri, Y. Structures of new cytotoxic antibiotics, piericidins c7 and c8. J. Antibiot. 2007, 60, 201.

(26) Wyche, T.P.; Hou, Y.; Vazquez-Rivera, E.; Braun, D.; Bugni, T.S. Peptidolipins bf, antibacterial lipopeptides from an ascidian-derived nocardia sp. J. Nat. Prod. 2012, 75, 735 .

(27) Zhang, Y.; Adnani, N.; Braun, D.R.; Ellis, G.A.; Barns, K.J.; Parker-Nance, S.; Guzei, I.A.; Bugni, T.S. Micromonohalimanes a and b: Antibacterial halimane-type diterpenoids from a marine micromonospora species. J. Nat. Prod. 2016, 79, 2968-2972.

(28) Horton, P.; Longley, R.; McConnell, O.; Ballas, L. Staurosporine aglycone (k252-c) and arcyriaflavin a from the marine ascidian, eudistoma sp. Cell. Mol. Life Sci. 1994, 50, 843845 .

(29) Andréo, M.A.; Jimenez, P.C.; Siebra, J.B.; Costa-Lotufo, L.V.; Vessecchi, R.; Niehues, M.; Lopes, J.L.; Lopes, N.P. Systematic uplc-esi-ms/ms study on the occurrence of staurosporine and derivatives in associated marine microorganisms from eudistoma vannamei. J. Braz. Chem. Soc. 2012, 23, 335-343.

(30) Leal, M.C.; Puga, J.; Serodio, J.; Gomes, N.C.M;, Calado, R.. Trends in the Discovery of New Marine Natural Products from Invertebrates over the Last Two Decades - Where and What Are We Bioprospecting? PLoS One, 2012, 7, e30580.

(31) Sherman, C. D., Hunt, A., Ayre, D. J. 2008. Is life history a barrier to dispersal? Contrasting patterns of genetic differentiation along an oceanographically complex coast. Biol. J. Linn. Soc., 95, 106-116.

(32) Hastings Point. Microsoft 2017. Available online: www.bing.com/maps (accessed 10/08/2017)

(33) Bolger, A.M.; Lohse, M.; Usadel, B. Trimmomatic: A flexible trimmer for illumina sequence data. Bioinformatics 2014, 30, 2114-2120.

(34) Masella, A.P.; Bartram, A.K.; Truszkowski, J.M.; Brown, D.G.; Neufeld, J.D. Pandaseq: Paired-end assembler for illumina sequences. BMC Bioinf. 2012, 13, 31.

(35) Caporaso, J.G.; Kuczynski, J.; Stombaugh, J.; Bittinger, K.; Bushman, F.D.; Costello, E.K.; Fierer, N.; Pena, A.G.; Goodrich, J.K.; Gordon, J.I. Qiime allows analysis of highthroughput community sequencing data. Nat. methods 2010, 7, 335-336.

(36) Küster, E.; Williams, S. Selection of media for isolation of streptomycetes. Nature 1964, 202, 928-929.

(37) English, A.L.; Boufridi, A.; Quinn, R.; Kurtböke, D. Evaluation of fermentation conditions triggering increased antibacterial activity from a near-shore marine intertidal environment-associated streptomyces species. Synth. Systems Biotechnol. 2017, 2, 28-38.

(38) Atlas, R.M. Handbook of microbiological media. CRC press: 2010.

(39) Williams, S.T.; Wellington, E.M.H. Actinomycetes. In Methods of soil analysis. Part 2. Chemical and microbiological properties, Page, A.L., Ed. American Society of Agronomy, Soil Science Society of America: Madison, WI, 1982; pp 969-987. 
(40) Wellington, E. Preservation of actinomycete inoculum in frozen glycerol. Microbios. Lett. 1979, 6, 151-157.

(41) Edgar, R.C. Muscle: Multiple sequence alignment with high accuracy and high throughput. Nucleic Acids Res. 2004, 32, 1792-1797.

(42) Wang, M.; Carver, J.J.; Phelan, V.V.; Sanchez, L.M.; Garg, N.; Peng, Y.; Nguyen, D.D.; Watrous, J.; Kapono, C.A.; Luzzatto-Knaan, T. Sharing and community curation of mass spectrometry data with global natural products social molecular networking. Nat. Biotechnol. 2016, 34, 828-837.

(43) Takada, K.; Ninomiya, A.; Naruse, M.; Sun, Y.; Miyazaki, M.; Nogi, Y.; Okada, S.; Matsunaga, S. Surugamides a-e, cyclic octapeptides with four d-amino acid residues, from a marine streptomyces sp.: Lc-ms-aided inspection of partial hydrolysates for the distinction of d-and l-amino acid residues in the sequence. J. Org. Chem. 2013, 78, 67466750 .

(44) Aoyagi, T.; Takeuchi, T.; Matsuzaki, A.; Kawamura, K.; Kondo, S.; Hamada, M.; Maeda, K.; Umezawa, H. Leupeptins, new protease inhibitors from actinomycetes. $J$. Antibiot.1969, 22, 283-286.

(45) McBrien, K.D.; Berry, R.L.; Lowe, S.E.; Neddermann, K.M.; Bursuker, I.; Huang, S.; Klohr, S.E.; Leet, J.E. Rakicidins, new cytotoxic lipopeptides from micromonospora sp. Fermentation, isolation and characterization. J. Antibiot. 1995, 48, 1446-1452.

(46) Martínez-García, M.; Díaz-Valdés, M.; Wanner, G.; Ramos-Esplá, A.; Antón, J. Microbial community associated with the colonial ascidian cystodytes dellechiajei. Environ. Microbiol. 2007, 9, 521-534.

(47) Tait, E.; Carman, M.; Sievert, S.M. Phylogenetic diversity of bacteria associated with ascidians in eel pond (woods hole, massachusetts, USA). J. Exp. Mar. Biol. Ecol. 2007, 342, 138-146.

(48) Behrendt, L.; Larkum, A.W.; Trampe, E.; Norman, A.; Sørensen, S.J.; Kühl, M. Microbial diversity of biofilm communities in microniches associated with the didemnid ascidian lissoclinum patella. ISME J. 2012, 6, 1222-1237.

(49) Morgan, J.L.; Darling, A.E.; Eisen, J.A. Metagenomic sequencing of an in vitrosimulated microbial community. PloS one 2010, 5, e10209.

(50) Sponga, F.; Cavaletti, L.; Lazzarini, A.; Borghi, A.; Ciciliato, I.; Losi, D.; Marinelli, F. Biodiversity and potentials of marine-derived microorganisms. J. Biotech. 1999, 70, 6569.

(51) Jensen, P.R.; Gontang, E.; Mafnas, C.; Mincer, T.J.; Fenical, W. Culturable marine actinomycete diversity from tropical pacific ocean sediments. Env. Microbiol. 2005, 7, 1039-1048.

(52) Penn, K.; Jensen, P.R. Comparative genomics reveals evidence of marine adaptation in salinispora species. BMC genomics 2012, 13, 86.

(53) Kurtböke, D.İ; Grkovic, T.; Quinn, R.J. Marine actinomycetes in biodiscovery. In Springer handbook of marine biotechnology, Springer: 2015; pp 663-676.

(54) Sunagawa, S.; Woodley, C.M.; Medina, M. Threatened corals provide underexplored microbial habitats. PloS one 2010, 5, e9554.

(55) Okami, Y.; Okazaki, T.; Kitahara, T.; Umezawa, H. Studies on marine microorganisms. V. J. Antibiot. 1976, 29, 1019-1025.

(56) Imada, C.; Koseki, N.; Kamata, M.; Kobayashi, T.; Hamada-Sato, N. Isolation and characterization of antibacterial substances produced by marine actinomycetes in the presence of seawater. Actinomycetologica 2007, 21, 27-31. 
(57) Kott, P. Ascidiacea (Tunicata) from deep waters of the continental shelf of Western Australia. J. Nat. Hist., 2008, 42, 1103-1217.

(58) Scherlach, K. \& Hertweck, C.. Triggering cryptic natural product biosynthesis in microorganisms. Org. Biomol. Chem., 2009, 7, 1753-1760. 


\section{SECTION 2}

\section{Tackling the issue of rediscovery and dereplication}

Natural products provide an immense wealth of structural complexity; however, natural product isolations and screening require a lot of time and effort. Furthermore, rediscovery of already known compounds is a major issue in this field of research. In Chapter 2, I focused my microbial isolation efforts on deriving actinomycetes from marine ascidians, a mostly untapped ecological source of biodiscovery, with the intention of enhancing the chemical diversity of natural products. This resulted in an actinomycete library of 120 diverse isolates. Thus, the challenge was to decide which isolates were most valuable for compound isolations. Recently, MS-based metabolomics approaches have been implemented for microbial strain prioritisation and several references were provided in the introduction to the thesis. Mass spectrometry is a very powerful analytical tool with high sensitivity and resolution; however, it does not provide a lot of insight about structural components in an extract. Therefore, it was my aim to develop a new approach using 2D NMR profiling for microbial strain selection which is presented in Chapter 3. In particular, I intended to investigate the questions, whether HSQC-TOCSY profiles can be used as a profiling tool to detect presence of specific structural classes in microbial extracts without the need of fractionation. Subsequently, whether this technique can be used as an approach to detect similarities and differences in microbial extracts and therefore be used for microbial strain dereplication.

Co-cultivation is a culturing method that can enhance chemical diversity as a result of interspecies interactions. In Chapter 4, four different microbial strains were co-cultured and the developed stain prioritisation approach (described in Chapter 3) was applied to dereplicate the obtained mono- and co-culture extracts. This provided further validation for the new 2D NMR profiling method. 


\section{CHAPTER 3 - HSQC-TOCSY NMR profiling: using informative metabolomics to prioritise microbial strains for natural product drug discovery}

\section{STATEMENT OF CONTRIBUTION TO CO-AUTHORED PAPER IN PREPARATION}

This chapter is a co-authored paper in preparation for submission. The bibliographic details of the co-authored paper, including all authors, are:

Buedenbender, L., Habener, L.J., Grkovic, T., Kurtböke, D.I., Duffy, S., Avery, V.M., Carroll, A.R., HSQC-TOCSY NMR profiling: using informative metabolomics to prioritise microbial strains for natural product drug discovery. Journal of Natural products, in preparation

The author this thesis, L. Buedenbender, developed the approach, performed microbial fermentations, extractions, chemical profiling; data analysis and prepared the manuscript. Leesa Habener assisted in revising the manuscript for important intellectual content. The co-authors $\mathrm{T}$. Grkovic, D. I. Kurtböke and A. R. Carroll are the supervisors of the $\mathrm{PhD}$ project and provided advice on the research design and feedback. S. Duffy and V. M. Avery performed antiplasmodial activity testing of the crude extracts.

(Signed)

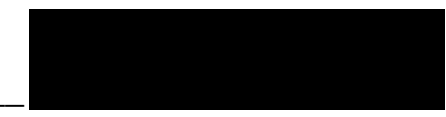

(Date) $\underline{01 / 09 / 2017}$

Larissa Buedenbender

(Countersigned) (Date) $\underline{31 / 08 / 2017}$

Corresponding author of paper: Anthony R. Carroll

(Countersigned)

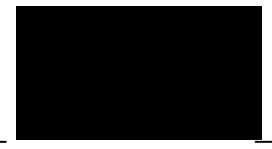

(Date) $\underline{31 / 08 / 2017}$

Principal supervisor: Anthony R. Carroll 


\subsection{Abstract}

Microbial natural products are still a promising source for drug leads due to their unique structural diversity and the possibility of laboratory based fermentations rather than harvesting organisms from the environment. However, rediscovery of already known natural products poses a great challenge and therefore, it is important to incorporate effective dereplication protocols early in natural product isolation efforts. A systematic approach for microbial strain prioritization for natural product discovery based on HSQC-TOCSY NMR profiles combined with biological activity of crude extracts was developed. NMR data provides structural information of the microbial extracts and can be used to target specific functional groups of natural products. The use of HSQC-TOCSY experiments offered increased chemical shift resolution by spreading the structural information onto two dimensions and thus permitted assessment of unfractionated extracts. Here, the utility of this approach was demonstrated to aid the selection of microbial strains with unique NMR profiles and potent anti-plasmodial activity from a library of 119 ascidian-associated actinomycete extracts that were prioritised for further natural product discovery investigations.

\subsection{Introduction}

Natural products and their derivatives continue to play an important role in the discovery and development of new therapeutical agents due to their unique structural diversity and chirality. ${ }^{1}$ Microorganisms represent one of the most important sources for new medicines. Furthermore recent evidence has shown that a significant number of invertebrate-derived natural product drug leads are actually produced by a microbial symbiont of the host species from which the natural product was originally isolated. ${ }^{2}$ With this in mind, microbial libraries, particularly derived from the marine environment, are expanding rapidly. Strains for drug discovery were traditionally selected based on morphology and/or biological activity of their crude extracts. This was then followed by bioassay-guided fractionation to isolate new bioactive molecules. The problem herein is that two microbial isolates could visually appear different but produce the same secondary metabolites, or alternatively appear the same morphologically yet produce different metabolites. Such aspects, as well as genuine duplication of isolates within a microbial strain library, have to be considered and thus new and innovative approaches are evolving to dereplicate cultured strains in order to overcome repeated re-isolation of the same metabolites.

Chemometric-metabolomics profiling approaches provide an overview of the expressed metabolites in extracts and aim to reduce redundancy. The two most common metabolomics 
profiling tools are LC-MS/MS and NMR profiling. The advantages of mass spectrometry are the high resolution and sensitivity, as well as the possibility to use MS/MS fragmentation. However, the technique is highly dependent on the ability of a compound to ionise and it is difficult to quantify and replicate the obtained results using different instruments and thus the technique is not universally applicable. NMR techniques, on the other hand, have much lower resolution and sensitivity compared to MS, but NMR is non-selective, non-destructive, and quantitative, it requires minimal sample handling and undeniably its greatest advantage is that it provides structural information about the constituents of the extracts. In recent years, NMR advancement has led to faster acquisition through optimised probes and stronger magnets, as well as automation and thus making this technique more powerful and more applicable for metabolomics studies. For this reason, NMR profiling was chosen as the preferred analytical technique in this study.

Microbial crude extracts are made up of potentially hundreds of metabolites, resulting in an immense signal overlap in the $1 \mathrm{D}{ }^{1} \mathrm{H}-\mathrm{NMR}$ spectrum. Therefore, implementation of $2 \mathrm{D}$ NMR techniques can increase chemical shift resolution by spreading the detected signals across two dimensions and thereby permitting assessment of unfractionated crude extracts. Twodimensional NMR experiments, such as TOCSY and HSQC, have previously been used for metabolite profiling of crude extracts. ${ }^{3,4}$ Herein, this chapter of the thesis aims to (1) develop the use of HSQC-TOCSY spectral profiles for characterisation of polyketide and peptide chemical diversity; (2) utilise HSQC-TOCSY spectral profiles in combination with biological activity to prioritise microbial strains from a library of 119 actinomycete crude extracts for natural product isolation efforts and (3) select a promising microbial strain to discovery bioactive natural products.

\subsection{Results \& Discussion}

\subsubsection{Determination of microbial culturing and chemical extraction protocol}

For the discovery of microbial natural products, it is of great importance to determine a suitable culture medium and metabolite extraction protocol. On the one hand, it is important to select a media type that offers enough nutrients for the culture to grow, but on the other hand it should not heavily contaminate the extracts with primary metabolites. Similarly, the extraction protocol should maximise the amount of extract, but should also be specific for the natural product containing part of the metabolome. Therefore, a range of culturing media and extraction techniques were compared to determine the most appropriate protocol for natural products 
discovery (Figure 1). Initially, a random selection of six different actinomycete strains was cultured on solid GYM + ASW and OMA agar plates as well as in liquid ASW-A media. The proton spectra of EtOAc extracts of both GYM + ASW and OMA solid media showed measurable yields of secondary metabolites. $\mathrm{MeOH}$ was also trialled as an extraction solvent, however, resulted in increased media elements in the extracts; particularly sugars and fatty acids contributed to the broadening and overlap of signals in ${ }^{1} \mathrm{H}-\mathrm{NMR}$ spectra. In liquid cultures, the actinomycete mycelia were separated from the media by centrifugation and cells were extracted in $\mathrm{MeOH}$. The liquid media phase was extracted by adsorbing onto $\mathrm{C}_{18}$ silica gel, which was subsequently eluted with $20 \%$ and $100 \% \mathrm{MeOH}$. The liquid media extracts contained only minute quantities of secondary metabolites and in the proton spectra, the metabolites of interest were shadowed by larger signals attributed to media components. Cell extracts revealed interesting secondary metabolite chemistry but overall resulted in significantly lower yields compared to those obtained through solid agar culture extractions. Consequently, solid GYM + ASW media was chosen for the cultivation of the entire actinomycete library.

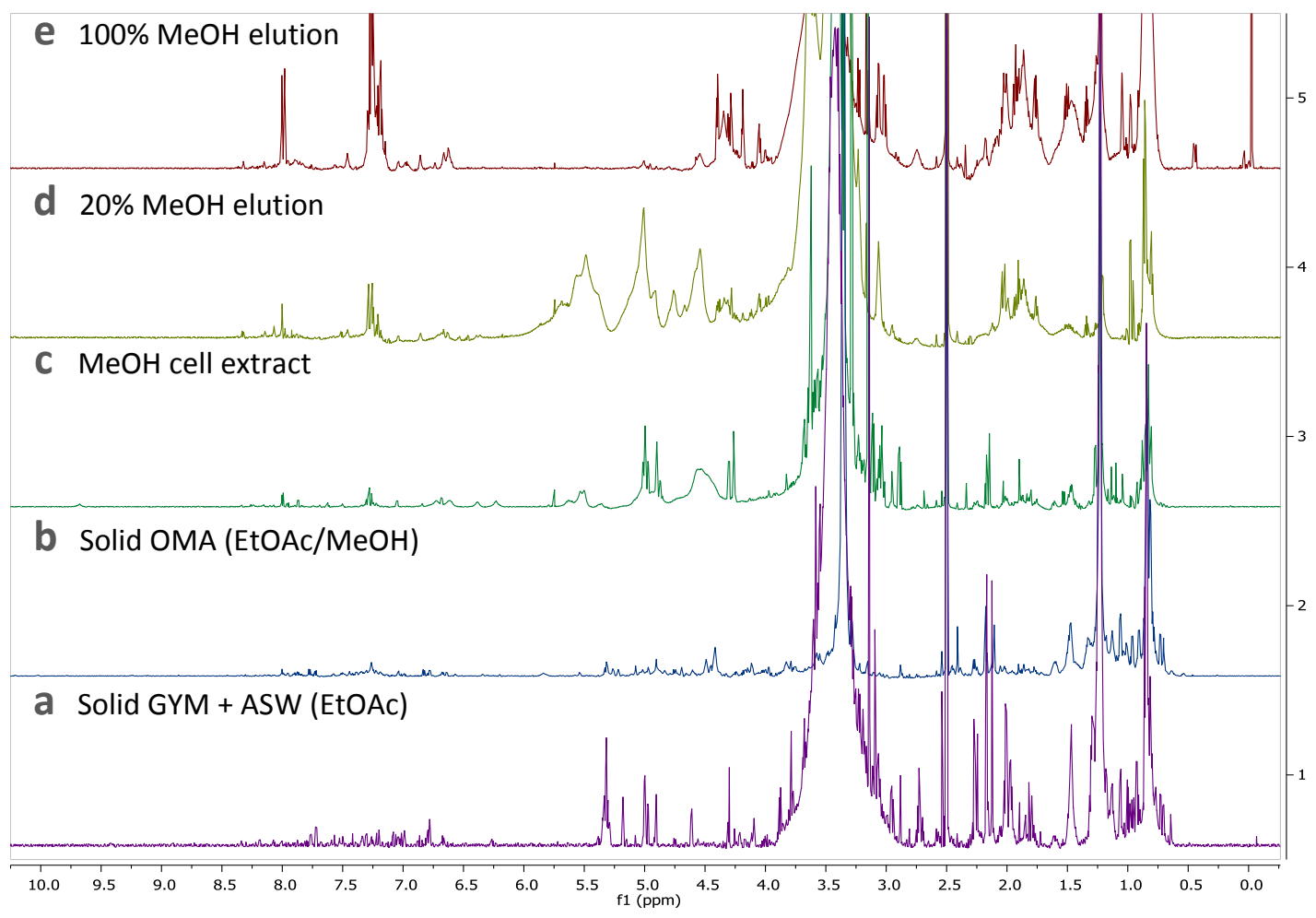

Figure 1. Evaluation of culturing and extraction methods for actinomycete isolate USC-16022. Normalised ${ }^{1} \mathrm{H}-\mathrm{NMR}$ spectra for each culturing and extraction condition, where f1 represents proton chemical shift in ppm. (a) EtOAc extraction of solid GYM + ASW medium. (b) EtOAc and $\mathrm{MeOH}$ extraction of solid OMA medium. (c) $\mathrm{MeOH}$ extract of cells from liquid culture. (d) $\mathrm{C}_{18}$ silica gel adsorption of liquid medium with $\mathrm{C}_{18}$ silica gel eluted with $20 \%$ $\mathrm{MeOH}$. (e) $\mathrm{C}_{18}$ silica gel adsorption of liquid medium with $\mathrm{C}_{18}$ silica gel eluted using $100 \%$ $\mathrm{MeOH}$. 


\subsubsection{Identification of regions and peaks of interest in 2D HSQC-TOCSY NMR spectra}

Microorganisms are capable of producing secondary metabolites of immense structural complexity (Figure 2). In bacteria, the majority of secondary metabolites are derived from polyketide synthase and non-ribosomal peptide synthases. ${ }^{5}$ These pathways utilise large protein complexes and have been studied extensively in the past two decades. ${ }^{6}$ The increased knowledge of biosynthetic pathways has opened the door for new development of sequencebased approached to natural product drug discovery. However, these techniques are not commonly available in natural products chemistry laboratories; therefore, an approach to utilise 2D HSQC-TOCSY NMR profiles to characterise polyketide and peptide chemical diversity was developed.

A HSQC-TOCSY NMR experiment is a 2D TOCSY experiment that is resolved into the carbon dimension. This shows through bond HSQC correlations of a proton to the carbon that it is attached to as well as further protonated carbons within the same spin system. ${ }^{7}$ In the traditional sense, this experiment is particularly useful for the elucidation of structures such as peptides, and to some extent polyketides, that typically display many overlapping proton signals. Spin fragments of these two structural classes occupy very specific chemical shift regions in HSQC-TOCSY spectra.
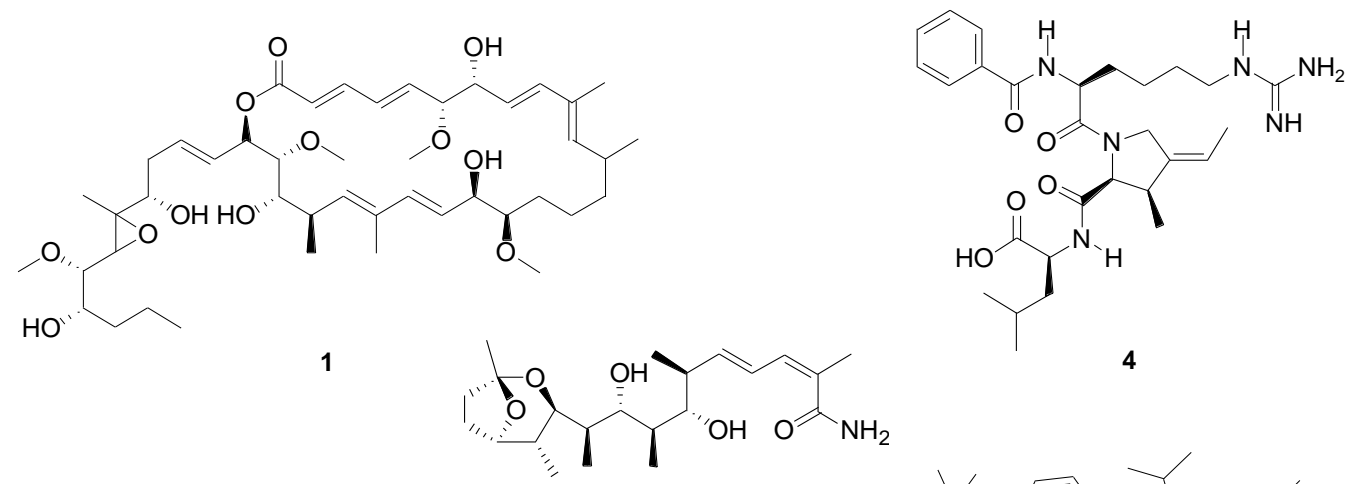

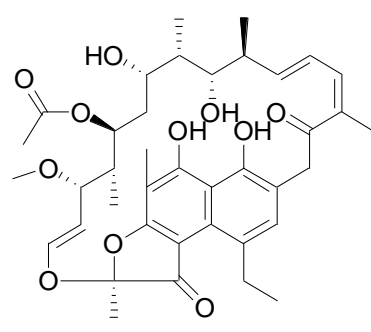

3

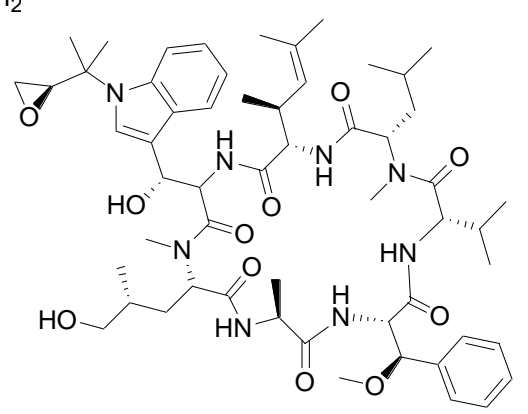

5

Figure 2. Examples of marine actinomycete derived polyketides and peptides: arenicolide A $(\mathbf{1})^{8}$, saliniketal A $(\mathbf{2})^{9}$, rifamycin B $(\mathbf{3})^{10}$, lucentamycin A $(\mathbf{4})^{11}$ and cyclomarin A $(\mathbf{5})^{12}$ 
The most characteristic features of polyketides are carbon-carbon chains that contain carbonyls on alternating carbons (Figure 2). These carbonyls are often reduced to alcohols or dehydrated to yield tri-substituted double bonds. ${ }^{13}$ The carbons adjacent to the hydroxy group display a characteristic chemical shift of $\delta_{\mathrm{C}} 60.0$ to $80.0 \mathrm{ppm} .{ }^{14}$ A HSQC-TOCSY spectrum allows observation of correlations between the methyl-protons, that generally have chemical shifts ranging from $\delta_{\mathrm{H}} 0.5-1.2 \mathrm{ppm}$, into the carbon at $\delta_{\mathrm{C}} 60.0-80.0 \mathrm{ppm}$ at the $\alpha$-position to the alcohol into (Figure 3). Both the $\alpha$-proton $\left(\delta_{\mathrm{H}} \sim 1.70 \mathrm{ppm}\right)$ and the $\beta$-proton $\left(\delta_{\mathrm{H}} \sim 1.50 \mathrm{ppm}\right.$ ) display correlations into the carbon at $\delta_{\mathrm{C}} 60.0-80.0 \mathrm{ppm}$. However, their peaks are generally less intense compared to those of methyl-protons. The utilisation of these the long-range HSQCTOCSY correlations of the proton $\left(\delta_{\mathrm{H}} 3.0-4.0 \mathrm{ppm}\right)$ bonded to the hydroxy carbon $\left(\delta_{\mathrm{C}} 60-\right.$ $80 \mathrm{ppm}$ ) are very useful to distinguish these signal from the similar correlations observed in hydroxy methines of sugars. Sugars are common by-products in microbial crude extracts, and occupy a region in the two-dimensional spectra that is prone to overcrowding of signals (Figure 3). The polyketide region of interest can be extended to include other typical polyketide moieties; for instance, when ethers or methoxy-groups are positioned next to the methyl, this results in a downfield shift of the adjacent carbons to $\delta_{C} 75-80 \mathrm{ppm}$. Further downfield shifts to approximately $\delta_{\mathrm{C}} 100 \mathrm{ppm}$ are indicative of the presence of dioxy-groups (Figure 3). Originally, the use of HMBC spectra was proposed, which would show correlations into the unprotonated carbons $\alpha$ to ketones, for the identification of polyketides in crude extracts; however, HMBC experiments are not as sensitive as HSQC-TOCSY experiments and would take an enormous amount of time for acquisition.

A similar approach can be taken to identify regions of interest in the spectra that are characteristic of peptides. If a deuterated NMR solvent is used, such as DMSO- $d_{6}$ that allows visualisation of exchangeable protons, characteristic HSQC-TOCSY correlations from an amide proton to $\alpha$ - and $\beta$-carbons can be observed. Amide protons typically display chemical shifts of $\delta_{\mathrm{H}} 6.0-10 \mathrm{ppm}$ and the absence of ${ }^{1} \mathrm{H}^{-13} \mathrm{C}$ HSQC correlations. ${ }^{14}$ In HSQC-TOCSY spectra, correlations through the spin system to carbons with shifts ranging from $\delta_{\mathrm{C}} 10-80 \mathrm{ppm}$ can be observed. Examples of partial peptide structures that depict such correlations are shown in Figure 3. Indoles are specific cases where HSQC-TOCSY correlations from the indole proton $\left(\delta_{\mathrm{H}} 10 \mathrm{ppm}\right)$ to aromatic carbon with resonances of approximately $\delta_{\mathrm{C}} 125 \mathrm{ppm}$ can be detected. ${ }^{14}$ Other heteroaromatic resonances occur even further downfield; imidazoles show a characteristic $\mathrm{N}-\mathrm{H}$ resonances of $\delta_{\mathrm{H}} 13.5 \mathrm{ppm}$ that show correlations to aromatic carbons resonating at $\delta_{\mathrm{C}} 135$ ppm. $^{14}$ 


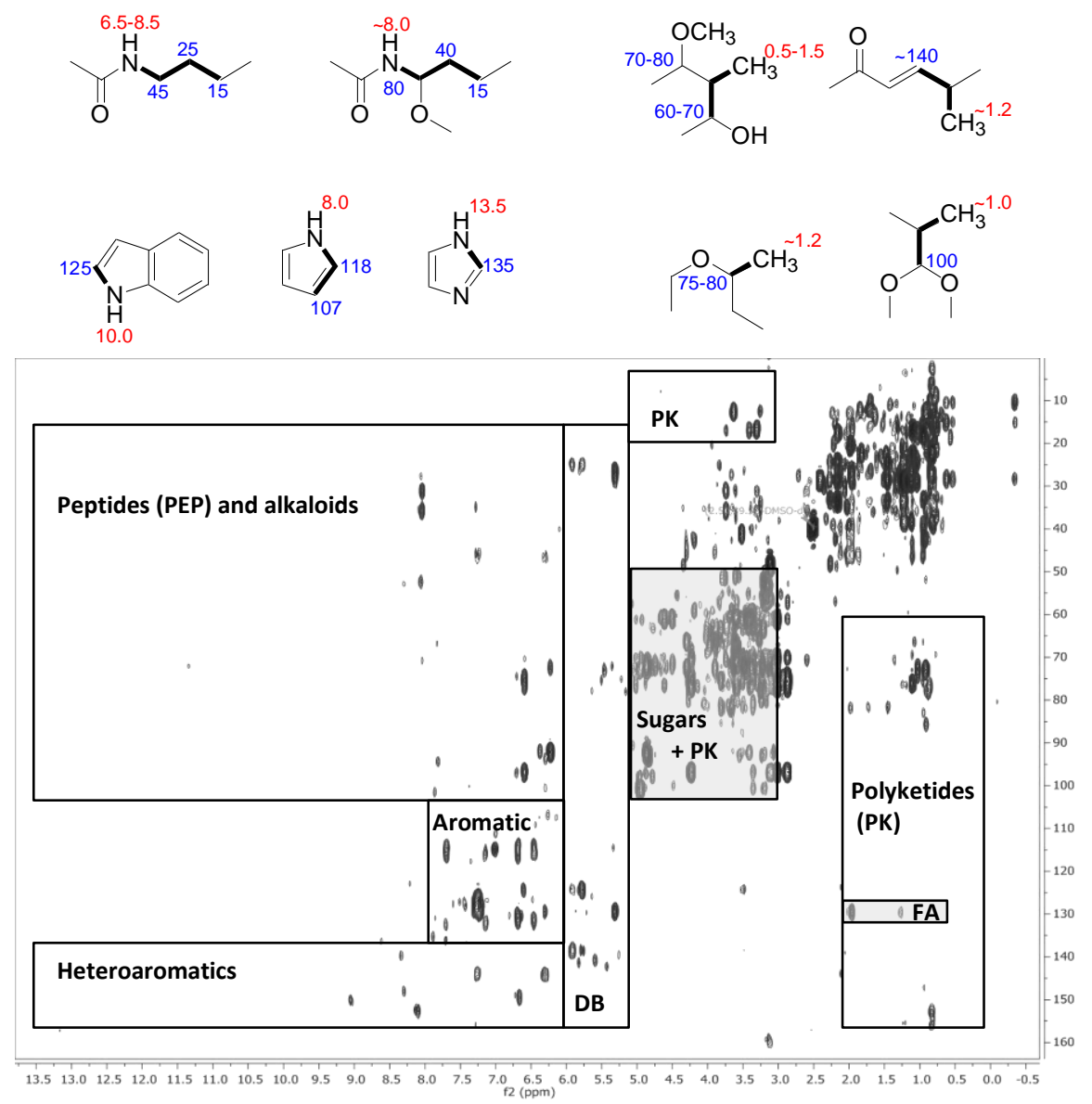

Figure 3. Regions of interest: HSQC-TOCSY spectrum highlighting chemical shift (ppm) regions that correspond to specific compound classes. Carbon chemical shifts are represented on $\mathrm{f} 1$ ( $\mathrm{ppm}$ ) and proton chemical shifts on $\mathrm{f} 2$ ( $\mathrm{ppm})$. Regions of interest include peptides (PEP), alkaloids, heteroaromatics, olfenic signals (DB), aromatics, polyketides (PK), sugars, and fatty acids (FA).

\subsubsection{Integrating metabolomics tools for microbial strain prioritisation from a library of 119 actinomycete extracts}

Metabolomics uses analytical chemometric techniques to identify and quantify the total metabolic processes of an organism. ${ }^{15}$ Thereby, chemical profiling data is analysed using multivariate statistics that allows detection of differential metabolites, identification of unique samples, and reduction of chemical redundancy. ${ }^{16}$ The theoretical workflow commences with microbial crude extracts that are then chemically profiled using spectroscopy (NMR) or spectrometry (MS) and are most often also screened for biological activity (Figure 4). The combined data is then statistically analysed to prioritise unique and chemically diverse samples for natural product isolations. Principal component analysis (PCA) and Partial Least Squares - 
Discriminatory Analysis (PLS-DA) are the two routinely used multivariate statistics in metabolomics. ${ }^{17}$ These techniques reduce large numbers of observed variables to fewer principal components that describe the variation between samples. ${ }^{18}$

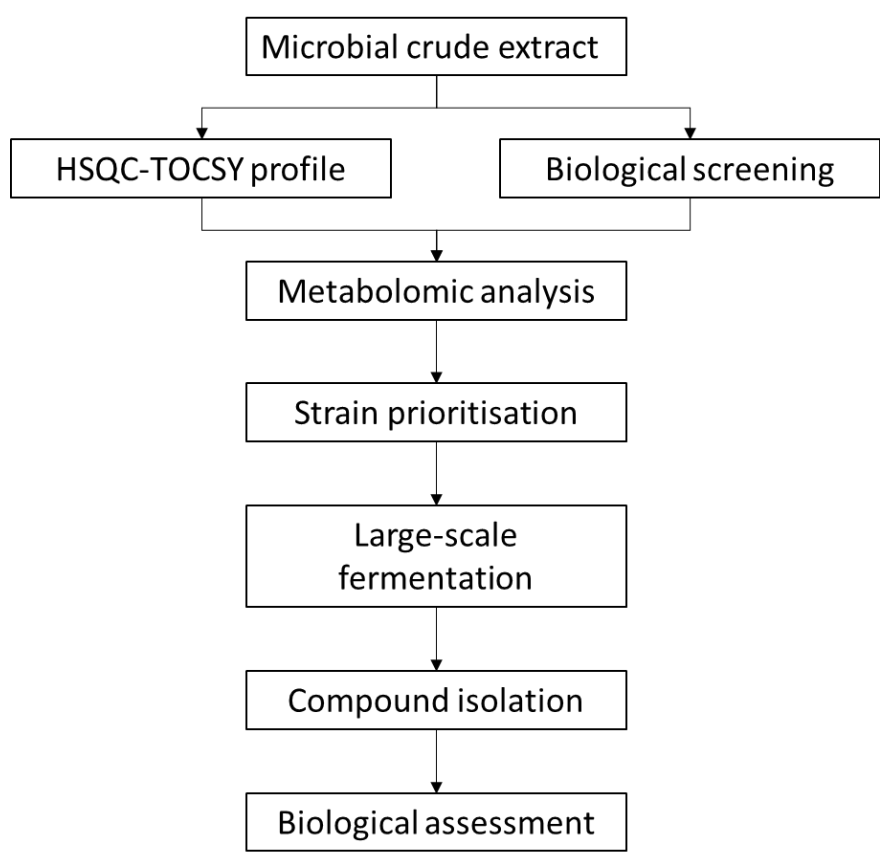

Figure 4. Workflow for metabolomics guided strain selection

The adapted metabolomics approach (Figure 4) was applied to dereplicate the acquired HSQC-TOCSY spectra for an actinomycete collection of 119 diverse isolates. One GYM + ASW agar culture plate per isolate was prepared and extracted in EtOAc after 2 weeks of incubation at $28{ }^{\circ} \mathrm{C}$. HSQC-TOCSY profiles were obtained and the crude extracts were tested for anti-plasmodial activity. Cross-peaks were picked from the regions of interest (detailed in Figure 3) and the data was exported to Excel, peaks were aligned by implementing an in-house macro allowing for a bin-threshold of $0.04 \mathrm{ppm}$ in the proton dimension and $0.4 \mathrm{ppm}$ in the carbon dimension. This resulted in a presence/absence matrix of a total of 1405 unique peaks from the 119 samples. This data is not actually suitable for PCA; and consequently, Principal Coordinate Analysis (PCoA) was chosen which can be used with similarity measures that are more suited for presence/absence data. The data was also subcategorised based on the partial structures derived from the HSQC-TOCSY polyketide (PK) and peptide (PEP) regions of interest, to reduce the numbers of observations in the multivariate analysis. When all peaks of interest (PoI) were included in the analysis, no clear clustering was observed in the PCoA plot, but there was a trend in the data based on the genus taxonomic level and the majority of active 
samples were observed to cluster closer together (Figure 5a/b). However, the first two principal coordinates accounted for $12 \%$ of the variation in the data, which indicates that the data set is comprised of very diverse samples. PCoAs were then individually performed for the polyketide and the peptide subcategories, which reduced the number of PoIs to 172 and 291, respectively (Figure 5c-f).

In the PCoA of polyketide PoIs, five clusters were observed (Figure 5c) and specific structural fragments could be associated to these clusters. The clusters 1,2 and 3 all contain specific $\mathrm{CH}_{3}$-CHOR fragments, while cluster 4 had very specific correlations from an upfield proton at $\delta_{\mathrm{H}} 1.23 \mathrm{ppm}$ to a downfield carbon at $\delta_{\mathrm{C}} 155 \mathrm{ppm}$ that is likely to be associated with a olefinic fragment. The first two dimensions the PCoA explained only $16 \%$ of the variation in the data, which essentially alludes to the high chemical diversity of the actinomycete collection. The remaining samples in cluster 5 contained a breadth of additional polyketide fragments with rather different HSQC-TOCSY profiles (Appendix II, Figure IIB contains PCoA plot with sample labels). In terms of natural products discovery a collection displaying chemical diversity is a desirable feature. There was no correlation found between samples that showed antiplasmodial activity and bioactive samples were present in each cluster of the polyketide analysis. However, samples that clustered on top of each other were found to contain the same or very similar polyketide resonances. Therefore, the PCoA was considered to be a useful tool for dereplication of identical samples. Nonetheless, HSQC-TOCSY spectra should always be consulted to confirm that two or more samples are identical, especially if the percent variance in the PCoA is low.

Three main clusters were observed for the principal coordinate analysis of the PEP PoI (Figure 5e). Cluster 1 contained only Micromonospora isolates that all had a specific HSQCTOCSY correlation from $\delta_{\mathrm{H}} 6.16 \mathrm{ppm}$ to $\delta_{\mathrm{C}} 91.8 \mathrm{ppm}$ in common; this correlation is likely to be an acetal type correlation and does not actually belong to a peptide. The various isolates in cluster 2 shared a correlation at $\delta_{\mathrm{H}} 6.58 \mathrm{ppm} / \delta_{\mathrm{C}} 75.9 \mathrm{ppm}$, while occurrence of another 38 PEP PoIs varied between the samples. From the broad cluster 3, it was not possible to discern any true correlations between the samples, neither inspection of the bioactive samples that all appeared to correlate revealed any particular PoI that contributed to their clustering (Figure 5f). 

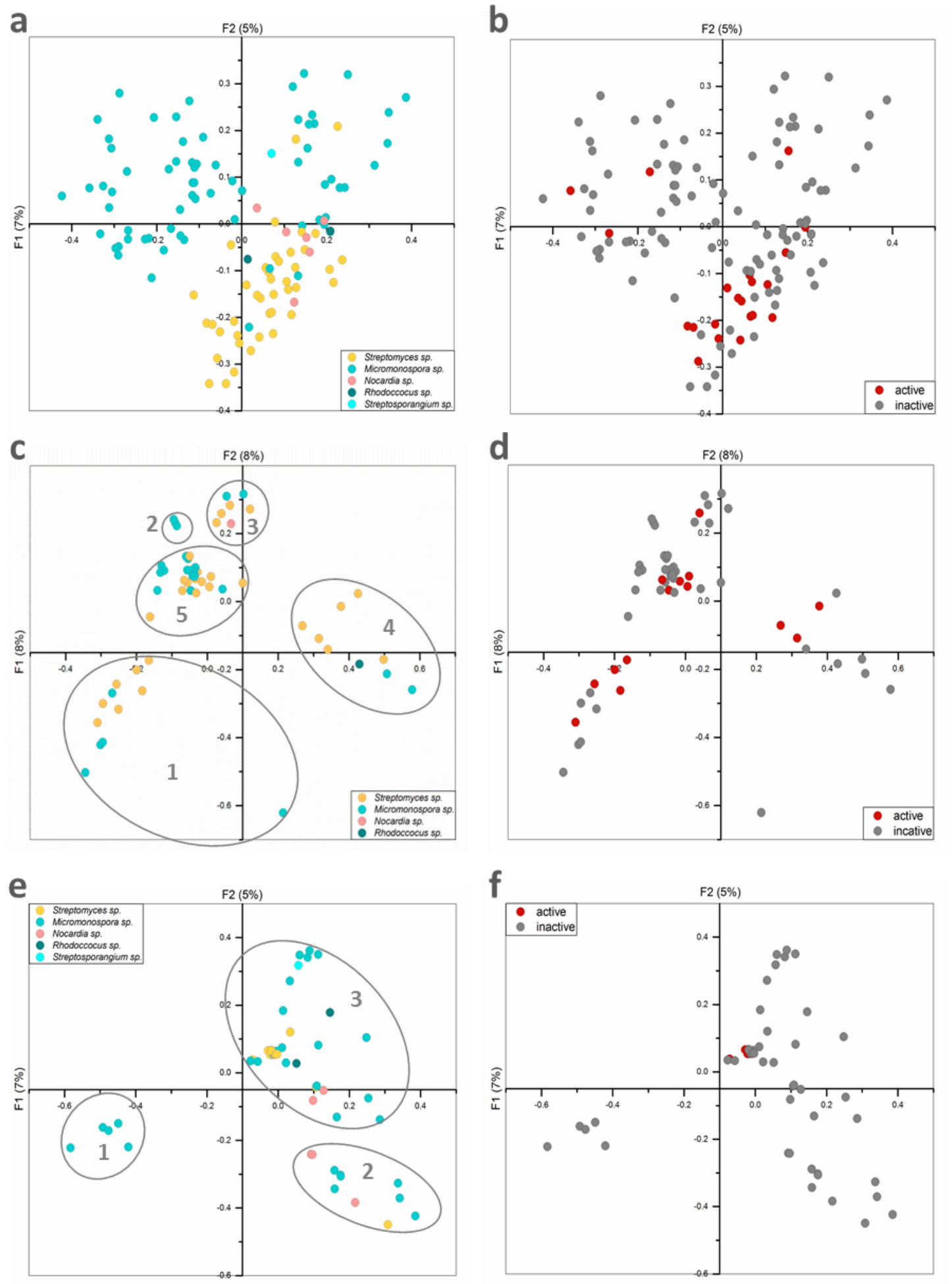

Figure 5. PCoA plots of HSQC-TOCSY data of 119 actinomycete stains; (a) for all PoI by genus (b) for all PoI by activity (c) for all PK by genus (d) for all PK by activity (e) for all PEP by genus (f) for all PEP by activity 
Of the 119 actinomycete isolates screened for anti-plasmodial activity, 18 demonstrated above $85 \%$ inhibition against Plasmodium falciparum at a tested concentration of $0.2 \mu \mathrm{g} / \mathrm{mL}$. Examination of respective HSQC-TOCSY spectra allowed detailed insight about the structural constituents of their extracts. First, all peaks of interest (PoI) were tallied, which averaged at 44 peaks for all active isolates (Figure 6). The highest number of PoI (88) was detected in the extract of Micromonspora sp. USC-16041 and it was clearly evident that Streptomyces sp. strains USC-16007 and USC-16025 metabolites were below the NMR detection limit, with only five and zero PoIs observed in the HSQC-TOCSY spectra, respectively. Such information is equally valuable as it reflects the lower ability of these strains to produce high amounts of secondary metabolites under the given culture conditions. It also suggests that the potency of the active compounds present in these strains are likely to be high. However further culture optimisations would be required for drug discovery efforts, therefore, these strains were not prioritised.

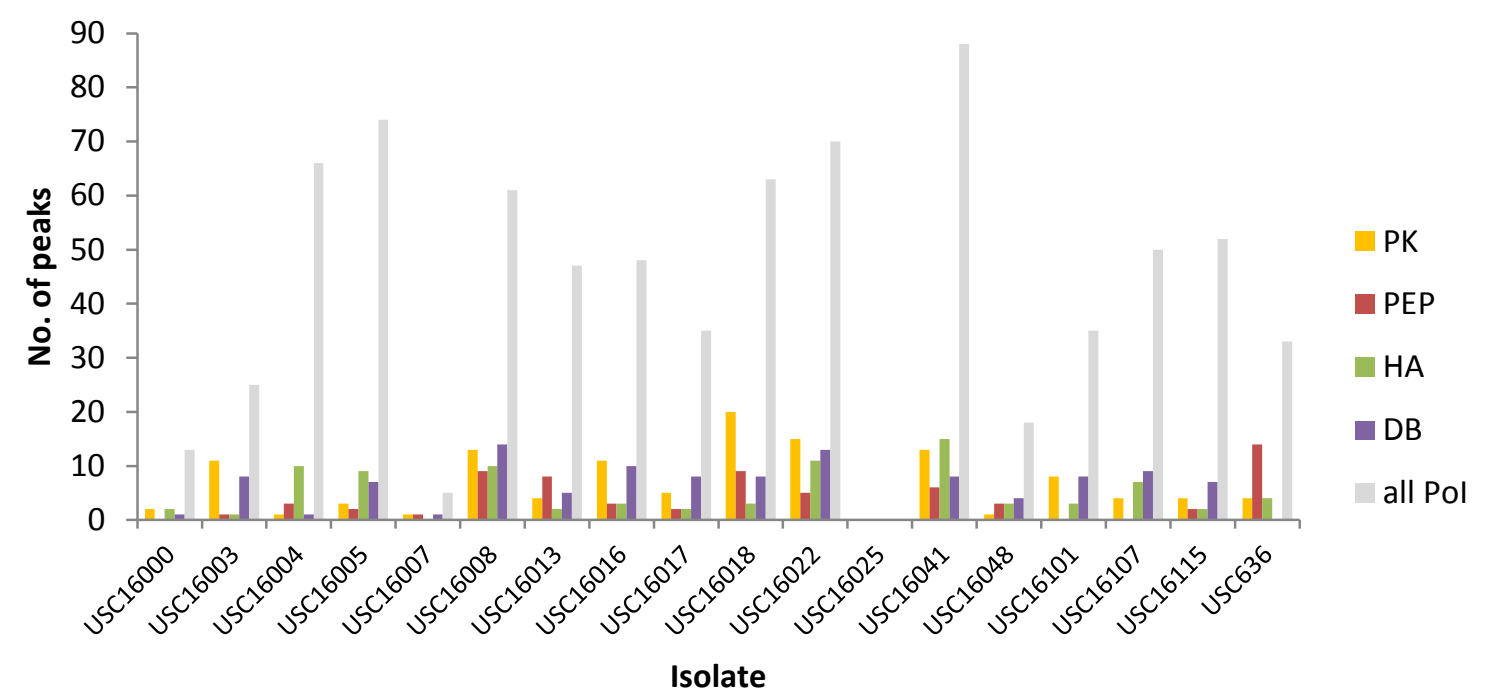

Figure 6. Account of HSQC-TOCSY peaks of interest (PoI) for each bioactive isolate $(\mathrm{PK}=$ polyketide $\mathrm{PEP}=$ peptide; $\mathrm{HA}=$ heteroaromatic, $\mathrm{DB}=$ olefinic $)$

Prioritisation of microbial strains for natural product drug discovery was first based on biological activity of the samples and high secondary metabolite signal diversity (total PoI) under the given culturing conditions was considered favourable, then also specific structural classes such as polyketides and peptides could be targeted. Lastly, PCoA was used to dereplicate the selected isolates to ensure that unique profiles were prioritised. Based on these criteria the order of prioritisation of isolates within a collection of 119 microbial isolates that are more likely to produce bioactive polyketide or peptides are detailed in Table 1. For natural product isolations, Streptomyces sp. USC-16018 was the number one choice if isolation of 
bioactive polyketides was desired. The strain, when grown on GYM + ASW solid media, had a total of 63 PoI associated with secondary metabolites, of which 20 diverse peaks were associated with polyketide-type compounds. In the PCoA, the isolate was positioned in 'cluster 1' but well away from any other sample (Figure 5c). USC-16041 and USC-16022 also scored high in the strain prioritisation approach (Table 1). Both isolates actually had higher total PoI diversity compared to USC-16018, however, their scores for polyketide and peptide associated PoI were lower. USC-636 and USC-16008 were better candidates for the isolation of peptides.

Table 1. Order of strain prioritisation based on bioactivity, high PK and PEP diversity and uniqueness of HSQC-TOCSY profile

\begin{tabular}{ccccccc|c}
\hline Isolate & Genus & $\begin{array}{c}\% \\
\text { inhibition }^{\text {a }}\end{array}$ & $\begin{array}{c}\text { total } \\
\text { PoI }\end{array}$ & $\begin{array}{c}\text { PK } \\
\text { PoI }\end{array}$ & $\begin{array}{c}\text { PEP } \\
\text { PoI }\end{array}$ & $\begin{array}{c}\text { PK+ } \\
\text { PEP }\end{array}$ & $\begin{array}{c}\text { Order of } \\
\text { prioritisation }\end{array}$ \\
\hline USC-16018 & Streptomyces & 94 & 63 & 20 & 9 & 29 & $\mathbf{1}$ \\
USC-16041 & Streptomyces & 93 & 88 & 13 & 6 & 19 & $\mathbf{2}$ \\
USC-16008 & Streptomyces & 96 & 61 & 13 & 9 & 22 & $\mathbf{3}$ \\
USC-16022 & Streptomyces & 71 & 70 & 15 & 5 & 20 & 4 \\
USC-636 & Streptomyces & 84 & 33 & 4 & 14 & 18 & 5 \\
USC-16016 & Streptomyces & 99 & 48 & 11 & 3 & 14 & 6 \\
USC-16003 & Streptomyces & 96 & 25 & 11 & 1 & 12 & 7 \\
USC-16013 & Streptomyces & 83 & 47 & 4 & 8 & 12 & 8 \\
USC-16101 & Streptomyces & 94 & 35 & 8 & 0 & 8 & 9 \\
USC-16017 & Streptomyces & 99 & 35 & 5 & 2 & 7 & 10 \\
USC-16005 & Streptomyces & 89 & 74 & 3 & 2 & 5 & 11 \\
USC-16115 & Streptomyces & 84 & 52 & 4 & 2 & 6 & 12 \\
USC-16004 & Streptomyces & 92 & 66 & 1 & 3 & 4 & 13 \\
USC-16107 & Micromonospora & 99 & 50 & 4 & 0 & 4 & 14 \\
USC-16048 & Micromonospora & 97 & 18 & 1 & 3 & 4 & 15 \\
USC-16000 & Streptomyces & 83 & 13 & 2 & 0 & 2 & 16 \\
USC-16007 & Streptomyces & 94 & 5 & 1 & 1 & 2 & - \\
USC-16025 & Streptomyces & 84 & 0 & 0 & 0 & 0 & - \\
\hline & & \multicolumn{7}{c}{$\%$-inhibition against P. falciparum at $2.0 \mu \mathrm{\mu g} / \mathrm{mL}$}
\end{tabular}

\subsubsection{Discovery of bioactive natural products from selected strains}

As a proof of concept, the microbial strain that exhibited the highest polyketide peak diversity was selected to investigate whether polyketide compounds could in fact be isolated from this strain. Fermentation of Streptomyces sp. strain USC-16018 was scaled up to 55 agar plates and extraction with EtOAc yielded $1.6 \mathrm{~g}$ of crude material. This extract was separated by reversed phase HPLC and natural product isolations were initially guided by ${ }^{1} \mathrm{H}$ NMR analysis of fractions. Isolations were guided by selecting fractions containing ${ }^{1} \mathrm{H}$ resonances between $\delta_{\mathrm{H}} 4.00$ and $6.00 \mathrm{ppm}$ indicative of oxygenated and olefinic protons potentially associated with polyketides. Short HSQC-TOCSY experiments (8 scans $=15$ minutes) of the selected fractions 
were acquired to confirm the presence of polyketides in the fractions. Fraction 46 was identified to contain a pure polyketide and a full set of two-dimensional NMR experiments $\left({ }^{1} \mathrm{H}-{ }^{1} \mathrm{H}\right.$ COSY, ${ }^{1} \mathrm{H}-{ }^{13} \mathrm{C}$ HSQC, and ${ }^{1} \mathrm{H}_{-}{ }^{13} \mathrm{C} \mathrm{HMBC}$ ) was obtained to elucidate the structure. The compound in fraction 46 turned out to be the known antibiotic dimer elaiophylin (6), which was previously reported to exhibit antibiotic and anti-plasmodial properties. ${ }^{19,20}$ The full structure elucidation of 6, as well as that of a new polyketide compound from the selected strain, is described in Chapter 5. It was of interest to confirm the presence of signals associated with this molecule in the original HSQC-TOCSY crude extract spectra of USC-16018. Nineteen elaiophylin-associated correlations were observed in the crude HSQC-TOCSY spectra (Figure 7).

Several indole-containing peptides with anti-plasmodial activity were previously isolated from the Streptomyces sp. strain USC-636. ${ }^{21}$ This isolate was included in the HSQC-TOCSY analysis as a control actinomycete strain and ranked fifth in the HSQC-TOCSY prioritisation approach. In comparison to the other active strains, USC-636 exhibited the most diverse

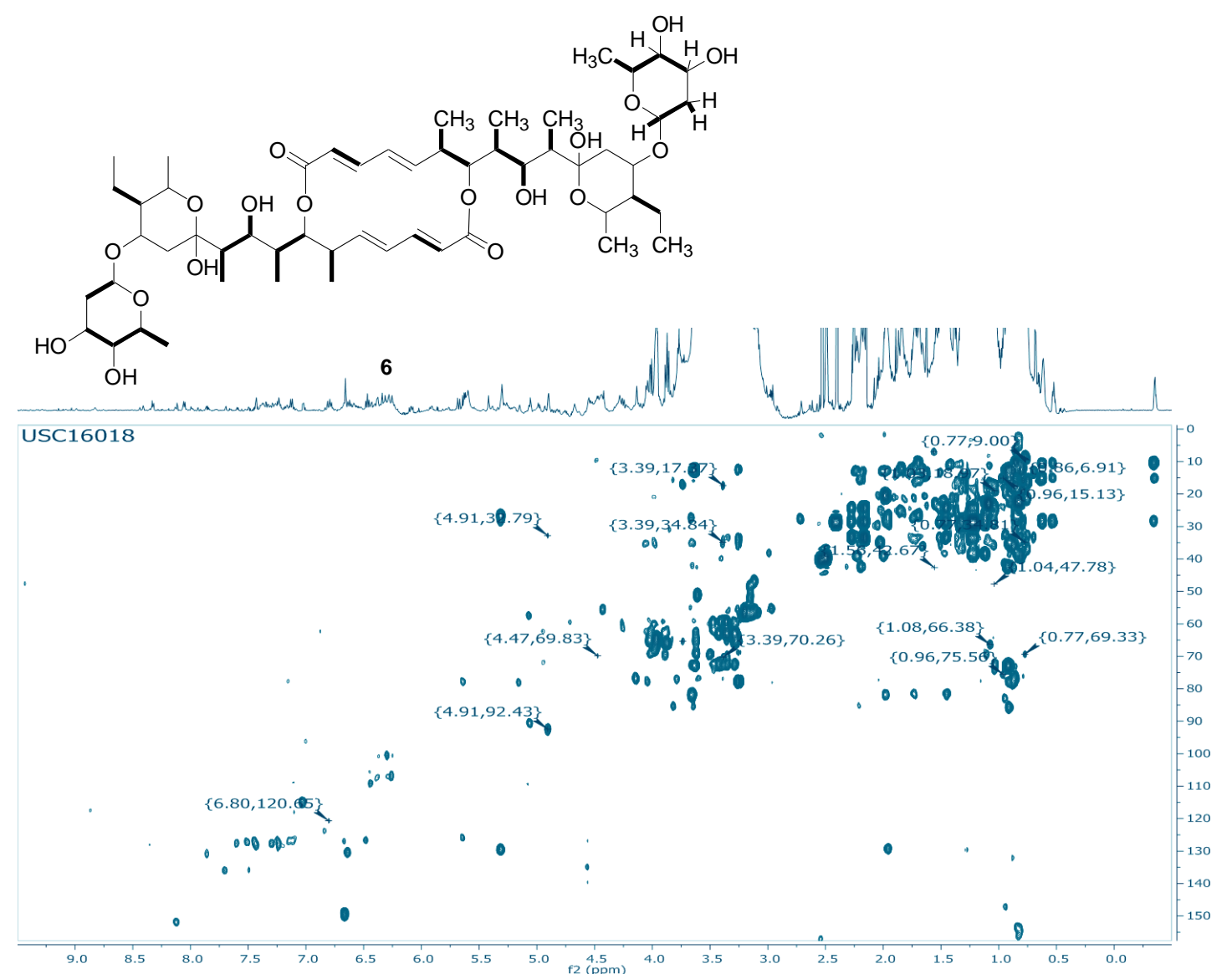

Figure 7. Elaiophylin correlations (labelled with ${ }^{1} \mathrm{H},{ }^{13} \mathrm{C}$ chemical shifts in ppm) observed in the crude HSQC-TOCSY spectra of strain USC-16018 (f1 representing ${ }^{13} \mathrm{C}$ chemical shifts in ppm and $\mathrm{f} 2$ representing ${ }^{1} \mathrm{H}$ chemical shift in ppm; HSQC-TOCSY correlations are shown in bold). 
peptide-associated HSQC-TOCSY peaks in the crude extract. Cross-peaks at $\delta_{\mathrm{H}} 10.78$ and $10.82 \mathrm{ppm} / \delta_{\mathrm{C}} 123.7 \mathrm{ppm}$ clearly identified the indole group found in naseseazines A - C (7 - 9, Figure 8). In all three structures the indole of one subunit was reduced to an indoline and correlations at $\delta_{\mathrm{H}} 7.11 / \delta_{\mathrm{C}} 123.4 \mathrm{ppm}$ could be observed in the crude spectrum.

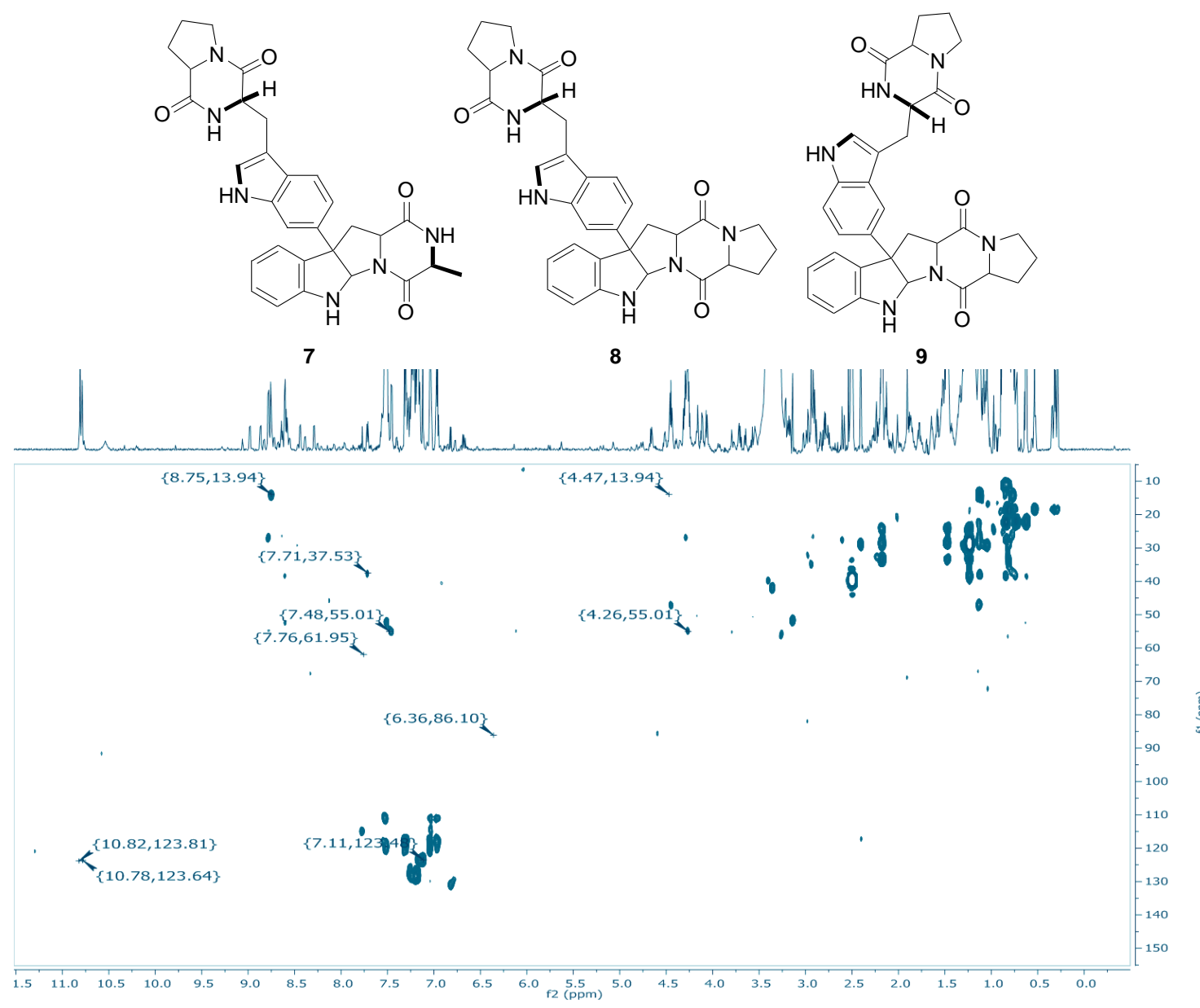

Figure 8. Naseseazine correlations (labelled with ${ }^{1} \mathrm{H},{ }^{13} \mathrm{C}$ chemical shift in ppm) observed in the crude HSQC-TOCSY spectra of strain USC-636 (f1 representing ${ }^{13} \mathrm{C}$ chemical shift in ppm, and $\mathrm{f} 2$ representing ${ }^{1} \mathrm{H}$ chemical shift in ppm; HSQC-TOCSY correlation are shown in bold).

\subsubsection{Detection limit of HSQC-TOCSY NMR spectra}

The compounds descibed above were isolated in low quantities ( $<1 \%$ of the crude extract), yet they could be detected in the HSQC-TOCSY spectra with 16 scans and 128 increments on a $800 \mathrm{MHz}$ NMR spectrometer equipped with a cryoprobe. In order to establish the actual detection limit of the HSQC-TOCSY profiles, a concentration curve was prepared using the commercially available polyketide antibiotic rifampicin (10, Figure 9). HSQC-TOCSY spectra 
were acquired under the same parameters as used for the microbial crude extracts. Average peak intensities for PK PoI were were established for 5.0, 2.5, 2.0, 1.5, and $1.0 \mathrm{mg} / \mathrm{mL}$ rifampicin (Table 2). Long-range HSQC-TOCSY correlations from the upfield methyls to the polyketide region of interest could be detected at concentrations as low as $1.5 \mathrm{mg} / \mathrm{mL}$ and the peak intesity of these polyketide fragments was used to establish a concentration curve (Figure 9). At $1.0 \mathrm{mg} / \mathrm{mL}(0.2 \mathrm{mg})$, only one-bond HSQC correlations of upfield protons were detected. One agar plate of an actinomycete culture on averaged yielded $15 \mathrm{mg}$ of extract and $0.3 \mathrm{mg}$ of this would equate to a $1.3 \%$ yield, which is a realistic yield for secondary metabolites in crude extracts.

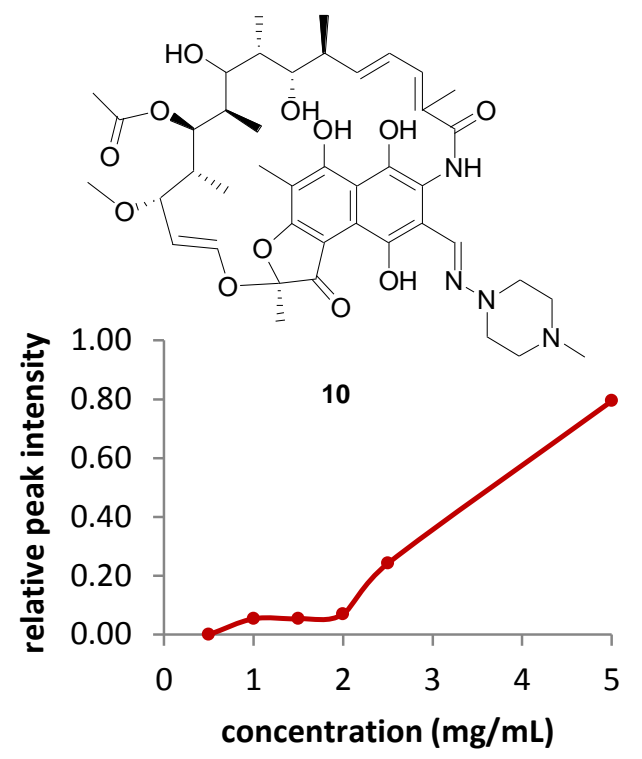

(a)

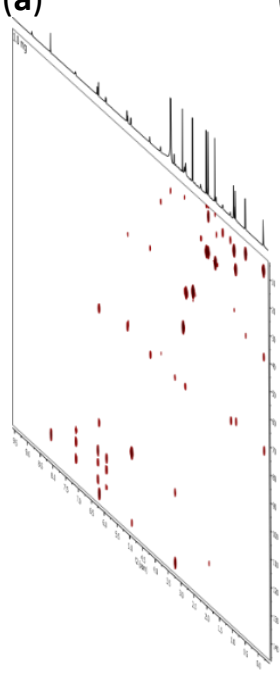

(b)

(c)

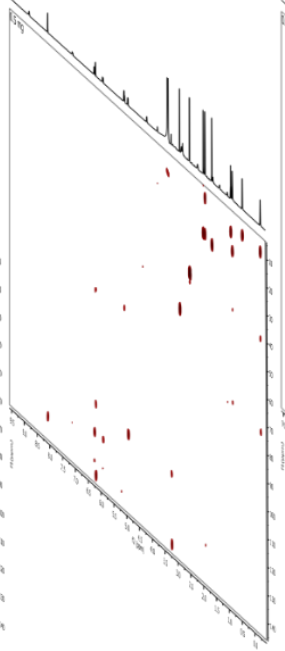

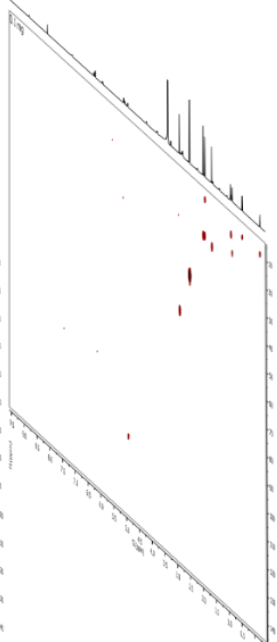

Figure 9. Rifampicin (10) concentration curve and corresponding HSQC-TOCSY spectra $\left(800 \mathrm{MHz}, 200 \mu \mathrm{L}\right.$ DMSO- $d_{6}, \mathrm{NS}=16$ ) (a) $5.0 \mathrm{mg} / \mathrm{mL}$ (b) $2.5 \mathrm{mg} / \mathrm{mL}$ (c) $1.0 \mathrm{mg} / \mathrm{mL}$

Table 2. Average PK PoI \%-intensities (relative to DMSO) for rifampicin standards at 5.0, 2.5, 2.0, 1.5, and $1.0 \mathrm{mg} / \mathrm{mL}$

\begin{tabular}{ccccc}
\hline $\begin{array}{c}\text { Concentration } \\
(\mathbf{m g} / \mathbf{m L})\end{array}$ & $\begin{array}{c}\text { \%-intensity at } \\
\boldsymbol{\delta}_{\mathbf{H}} \mathbf{- 0 . 2 6} / \boldsymbol{\delta}_{\mathbf{C}} \mathbf{7 3 . 4}\end{array}$ & $\begin{array}{c}\text { \%-intensity at } \\
\boldsymbol{\delta}_{\mathbf{H}} \mathbf{1 . 0 3 /} \boldsymbol{\delta}_{\mathbf{C}} \mathbf{7 3 . 4}\end{array}$ & $\begin{array}{c}\text { \%-intensity at } \\
\boldsymbol{\delta}_{\mathbf{H}} \mathbf{0 . 8 3 / \boldsymbol { \delta } _ { \mathbf { C } } \mathbf { 7 2 . 0 }}\end{array}$ & Average peak \%-intensity \\
\hline 5.0 & 1.2 & 0.50 & 0.69 & 0.79 \\
2.5 & 0.46 & 0.15 & 0.12 & 0.24 \\
2.0 & 0.072 & 0.067 & - & 0.070 \\
1.5 & 0.059 & 0.050 & - & 0.055 \\
1.0 & 0.046 & 0.046 & & 0.046 \\
0.5 & - & - & - & 0.00 \\
\hline
\end{tabular}




\subsubsection{Limitations and considerations}

A limitation of this approach is the requirement of a NMR spectrometer with a high-field strength magnet. The experiments in this study were performed on a Bruker $800 \mathrm{MHz}$ NMR spectrometer equipped with a cryoprobe and on average the HSQC-TOCSY approach proved useful. Acquisition using the same parameters on a $500 \mathrm{MHz}$ NMR spectrometer did not provide enough sensitivity. Even when acquired on the $800 \mathrm{MHz}$ spectrometer, extracts with low yields $(<4 \mathrm{mg}$ of crude extract) did not show many correlations in the spectra. The tradeoff when using a lower frequency magnet is the increased number of scans required to obtain the same spectrum sensitivity. Depending on the number of isolates to be screened, increased acquisition time might be a feasible option in order to improve detection. Reduction of the relaxation delay between scans to 0.8 seconds further enables shortening of acquisition time without overly compromising the sensitivity of the spectrum (except of protons with long spinlattice relaxations $\left.-\mathrm{T}_{1}\right){ }^{22}$ Another way to overcome the issue of low detection would be the use of Shigemi NMR tubes that require smaller amounts of NMR solvent and therefore result in increased concentration of the sample.

The advantage of working with microorganisms is that their secondary metabolism can be manipulated through small modifications of the culture media. ${ }^{23}$ The one-strain-manycompounds (OSMAC) principle is commonly used to diversify chemical extracts of microorganisms. HSQC-TOCSY spectral profiling is a useful way to determine which cultivation media and conditions are most successful at triggering secondary metabolite production. Figure 10 represents how cultivation of Streptomyces sp. strain USC-16022 on GYM + ASW compared to OMA media produced a greater diversity of secondary metabolites. It appears that GYM + ASW media particularly triggered the synthesis of peptides and a lot more aromatic peaks can be observed. A further solution to enhance peak detection by NMR would be media enrichment with ${ }^{13} \mathrm{C}$-labelled sugars, amino acids or propionate.

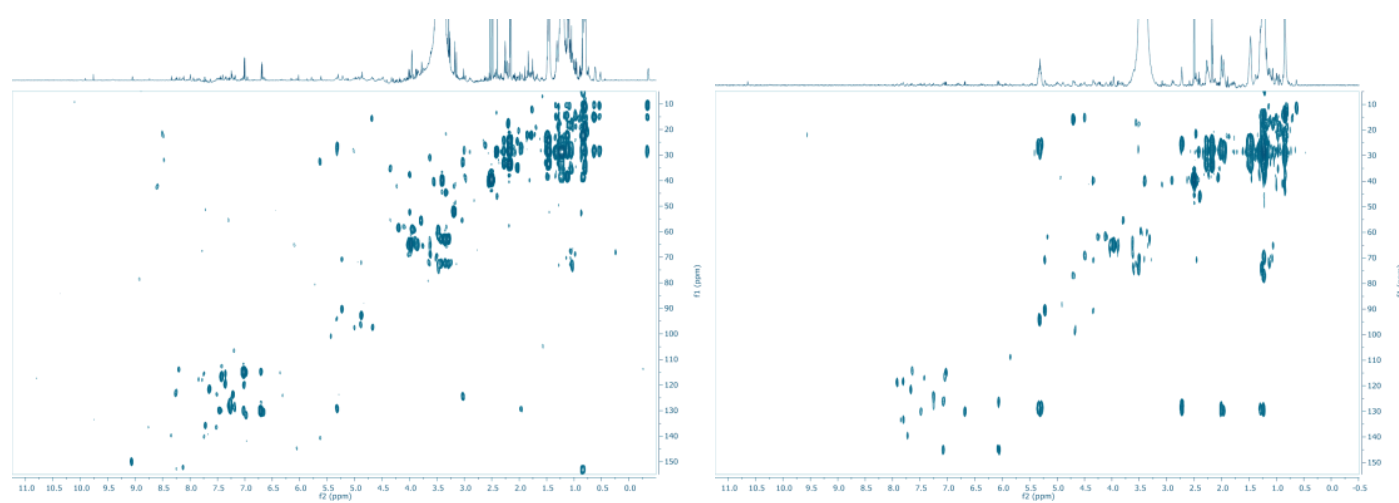

Figure 10. Representation of OSMAC for strain USC-16022 on (a) GYM + ASW and (b) OMA extracts 


\subsection{Conclusion}

A new approach to detect specific natural product structural fragments in HSQC-TOCSY NMR spectra of microbial crude extracts was developed. These HSQC-TOCSY profiles together with biological activity and subsequent dereplication through PCoA was used to prioritise microbial strains that have a higher potential to produce bioactive secondary metabolites. The successful application of this technique was exemplified though the selection of a promising microbial strain USC-16018 from a library of 119 actinomycete crude extracts that lead to the isolation of a polyketide with potent anti-plasmodial activity. This adds to the repertoire of strain selection/dereplication approaches and it is the first time that HSQC-TOCSY NMR spectra have been used for this purpose. These findings highlight the great potential of NMR for chemical profiling.

\subsection{Experimental section}

\subsubsection{Sample collection and microbial strain isolations}

One hundred and twenty actinomycete strains were isolated from three Australian ascidians, Symplegma rubra, Aplidium solidium and Polyclinum vasculosum as previously described in Chapter 2. One marine sediment derived actinomycete isolate (USC-636) with previously assessed chemistry was also included in the analysis. ${ }^{21}$

\subsubsection{Establishment of microbial extract library}

One hundred and twenty-one actinomycete isolates were incubated on both oatmeal agar (OMA; $20 \mathrm{~g}$ oatmeal, $20 \mathrm{~g}$ bacteriological agar, $3 \mathrm{~g}$ yeast extract, $1 \mathrm{~L}$ deionised $\mathrm{H}_{2} \mathrm{O}$ ) and glucose yeast malt agar plates (GYM + ASW; adapted from DSMZ Medium 65 https://www.dsmz.de/microorganisms/medium/pdf/DSMZ_Medium65.pdf) for 2 weeks at 28 ${ }^{\circ} \mathrm{C}$. Two of actinomycete isolates (USC-16072 and USC-16114) could not be revived from the glycerol stocks and were excluded from the analysis. After two weeks of incubation, the agar cultures were transferred to a $100 \mathrm{~mL}$ Schott bottle and suspended in EtOAc, terminating the growth of the culture and commencing chemical extraction. The culture plates were blended with a conventional kitchen stick blender and left on a rotary shaker at $200 \mathrm{rpm}$ overnight. The EtOAc extracts were decanted into clean test tubes and dried under vacuum in a centrifugal evaporator (GeneVac Technologies HT-12). The extracts were resuspended in $\mathrm{MeOH}$, filtered $(0.2 \mu \mathrm{m})$, and again dried under vacuum. Yields of crude extract per culture plate varied 
between $0.8-68 \mathrm{mg}$. For NMR spectroscopy, the unfractionated extracts, as well as media blanks, were suspended in $200 \mu \mathrm{L}$ DMSO- $d_{6}$ and transferred to $3 \mathrm{~mm}$ NMR tubes.

The ${ }^{1} \mathrm{H}$ NMR data was acquired under following parameters: number of scans $16,{ }^{1} \mathrm{H} 90^{\circ}$ pulse $8.4 \mu \mathrm{sec}$, interscan relaxation delay $0.8 \mathrm{sec}$, spectral width $20.0294 \mathrm{ppm}$, transmitter centre 6.175 ppm, O1 4940.62 (acquisition time $58 \mathrm{sec}$ ). HSQC-TOCSY spectra were obtained at: number of scans $16,{ }^{1} \mathrm{H} 90^{\circ}$ pulse $8.4 \mu \mathrm{sec}$, interscan relaxation delay $0.8 \mathrm{sec}$, increments 128, spectral width $16.0235 \mathrm{ppm}$, transmitter centre $6.000 \mathrm{ppm}, \mathrm{O} 14800.6 \mathrm{~Hz}, \mathrm{~F} 1$ spectral width 170 ppm, F1 transmitter centre 80.000 ppm, spinlock mixing time $0.06 \mu$ sec.

\subsubsection{Anti-plasmodial testing}

P. falciparum parasites (3D7 and Dd2 strains) were grown in RPMI 1640 supplemented with $25 \mathrm{mM}$ HEPES, $5 \%$ AB human male serum, $2.5 \mathrm{mg} / \mathrm{mL}$ Albumax II, and $0.37 \mathrm{mM}$ hypoxanthine. Initially, the parasites were subjected to two rounds of sorbitol synchronisation. After which ring stage parasites were exposed to the microbial crude extracts at concentrations of $200,20,2$, and $0.2 \mu \mathrm{g} / \mathrm{mL}$ in 384-wells imaging microplates (PerkinElmer CellCarrier), as previously described. ${ }^{24}$ Plates were incubated for $72 \mathrm{~h}$ at $37{ }^{\circ} \mathrm{C}, 90 \% \mathrm{~N}_{2}, 5 \% \mathrm{CO}_{2}, 5 \% \mathrm{O}_{2}$, following incubation parasites were stained with 2-(4-amidinophenyl)-1H-indole-6carboxamidine (DAPI), and imaged using an Opera QEHS micro-plate confocal imaging system (PerkinElmer). Images were analysed as previously described by Duffy and Avery. ${ }^{24}$

\subsubsection{Data processing and Multivariate analysis}

In MestReNova 11.0, proton spectra were corrected for phase and baseline and referenced to the DMSO- $d_{6}$ solvent peak $\left(\delta_{\mathrm{H}} 2.50 \mathrm{ppm}\right.$ ). For processing of HSQC-TOCSY spectra, $\mathrm{t} 2$ noise was reduced, apodisation was set to $0.00^{\circ}$ sine bell, $10.00 \mathrm{~Hz}$ Gaussian and $50.0 \%$ Sine Bell II and Sine Square II in the $\mathrm{f} 2$ dimension and to $90^{\circ}$ sine bell in $\mathrm{f} 1$; spectra were again referenced to the DMSO- $d_{6}$ solvent peak in both dimensions $\left(\delta_{\mathrm{H}} 2.50 \mathrm{ppm}\right.$ and $\left.\delta_{\mathrm{C}} 39.51 \mathrm{ppm}\right)$. Cross peaks were picked by zooming in to the regions of interest setting peak picking to manual threshold with the peak picking options set to 4.00 sensitivity and maximum number of peaks 1000 and dragging the cursor over the spectra. For polyketide signals this included the region from $\delta_{\mathrm{H}}$ $0.00-2.00 \mathrm{ppm}$ and from $\delta_{\mathrm{C}} 60.0-160.0 \mathrm{ppm}$, for peptides from $\delta_{\mathrm{H}} 6.00-14.00 \mathrm{ppm}$ and $\delta_{\mathrm{C}}$ $10.0-100.0 \mathrm{ppm}$, and for heteroaromatics and double bonds with electron withdrawing atoms between $\delta_{\mathrm{H}} 5.00-14.00 \mathrm{ppm}$ and $\delta_{\mathrm{C}} 135-160 \mathrm{ppm}$. If several spectra are selected, MestReNova allows peak picking to be performed in parallel on all selected spectra. All 2D peaks were exported as text files and peak data was tabulated in Excel (Microsoft Office 2010). Using an Excel macro, peaks between samples were aligned allowing for an error threshold of 
$0.04 \mathrm{ppm}$ for proton shifts and $0.4 \mathrm{ppm}$ for carbon chemical shifts. A Dice similarity matrix was then established and used for Principal Coordinate Analysis (PCoA).

\subsubsection{Determination of HSQC-TOCSY spectra detection limit}

Rifampicin (SigmaAldrich) standards were prepared at 5.0, 2.5, 2.0, 1.5, and $1.0 \mathrm{mg} / \mathrm{mL}$ in DMSO- $d_{6}$ and transferred to $3 \mathrm{~mm}$ NMR tubes. HSQC-TOCSY spectra were acquired under the same parameters as stated above for the microbial crude extracts. Peaks in the polyketide region of interest $\left(\delta_{\mathrm{H}}-0.50-2.00 \mathrm{ppm}\right.$ and $\left.\delta_{\mathrm{C}} 60-155 \mathrm{ppm}\right)$, as well as the DMSO solvent peak $\left(\delta_{\mathrm{H}} 2.50 / \delta_{\mathrm{C}} 39.51\right)$ were picked in MestReNova 11.0 and exported to Excel. For each concentration standard, the average peak intensity of polyketide associated peaks were determined relative to the DMSO solvent peak and plotted as a concentration curve.

\subsection{References}

(1) Newman, D. J.; Cragg, G. M. J. Nat. Prod. 2016, 79, 629.

(2) Piel, J. Curr. Med. Chem. 2006, 13, 39.

(3) Robinette, S. L.; Ajredini, R.; Rasheed, H.; Zeinomar, A.; Schroeder, F. C.; Dossey, A. T.; Edison, A. S. Anal. Chem. 2011, 83, 1649.

(4) Ben, H.; Ragauskas, A. J. Energy Fuels 2011, 25, 5791.

(5) Fischbach, M. A.; Walsh, C. T. Chem. Rev. 2006, 106, 3468.

(6) Staunton, J.; Weissman, K. J. Nat. Prod. Rep. 2001, 18, 380.

(7) Kover, K.; Prakash, O.; Hruby, V. J. Magn. Reson., Ser. A 1993, 103, 92.

(8) Williams, P. G.; Miller, E. D.; Asolkar, R. N.; Jensen, P. R.; Fenical, W. J. Org. Chem. 2007, 72, 5025.

(9) Williams, P. G.; Asolkar, R. N.; Kondratyuk, T.; Pezzuto, J. M.; Jensen, P. R.; Fenical, W. J. Nat. Prod. 2007, 70, 83.

(10) Kim, T. K.; Hewavitharana, A. K.; Shaw, P. N.; Fuerst, J. A. Appl. Environ. Microbiol. 2006, 72, 2118.

(11) Cho, J. Y.; Williams, P. G.; Kwon, H. C.; Jensen, P. R.; Fenical, W. J. Nat. Prod. 2007, $70,1321$.

(12) Renner, M. K.; Shen, Y.-C.; Cheng, X.-C.; Jensen, P. R.; Frankmoelle, W.; Kauffman, C. A.; Fenical, W.; Lobkovsky, E.; Clardy, J. J. Am. Chem. Soc. 1999, 121, 11273.

(13) McMurry, J. Organic Chemistry: With Biological Applications; Brooks/Cole, 2010.

(14) Pretsch, E.; Bühlmann, P.; Affolter, C.; Pretsch, E.; Bhuhlmann, P.; Affolter, C. Structure determination of organic compounds; Springer, 2009; Vol. 13.

(15) Rochfort, S. J. Nat. Prod. 2005, 68, 1813.

(16) Wu, C.; Kim, H. K.; van Wezel, G. P.; Choi, Y. H. Drug Discovery Today: Technol. 2015, 13, 11 .

(17) Worley, B.; Powers, R. Curr. Metabolomics 2013, 1, 92. 
(18) Ali, K.; Iqbal, M.; Yuliana, N. D.; Lee, Y.-J.; Park, S.; Han, S.; Lee, J.-W.; Lee, H.-S.; Verpoorte, R.; Choi, Y. H. Metabolomics 2013, 9, 778.

(19) Arcamone, F.; Bertazzoli, C.; Ghione, M.; Scotti, T. G. Microbiol. 1959, 7, 207.

(20) Supong, K.; Sripreechasak, P.; Tanasupawat, S.; Danwisetkanjana, K.; Rachtawee, P.; Pittayakhajonwut, P. Appl. Microbiol. Biotechnol. 2017, 101, 533.

(21) Buedenbender, L.; Grkovic, T.; Duffy, S.; Kurtböke, D. I.; Avery, V. M.; Carroll, A. R. Tetrahedron Lett. 2016, 57, 5893.

(22) Zani, C. L.; Carroll, A. R. J. Nat. Prod. 2017, 80, 1758.

(23) Bode, H. B.; Bethe, B.; Höfs, R.; Zeeck, A. ChemBioChem 2002, 3, 619.

(24) Duffy, S.; Avery, V. M. Am. J. Trop. Med. Hyg. 2012, 86, 84. 


\section{CHAPTER 4 - Detecting chemical diversity: HSQC- TOCSY NMR spectra show co-cultivation-induced secondary metabolite production}

\section{STATEMENT OF CONTRIBUTION TO CO-AUTHORED PAPER IN PREPARATION}

This chapter is a co-authored paper in preparation for submission. The bibliographic details of the coauthored paper, including all authors, are:

Buedenbender, L., Kurtböke, D.I., Carroll, A.R., Detecting chemical diversity: HSQC-TOCSY NMR spectra show co-cultivation-induced secondary metabolite production, in preparation

The author this thesis, L. Buedenbender, designed and performed all experimental work, data analysis and prepared the manuscript. The co-authors D. I. Kurtböke and A. R. Carroll are the supervisors of the $\mathrm{PhD}$ project and provided advice on the research design and feedback.

(Signed) (Date) $\underline{01 / 09 / 2017}$

Larissa Buedenbender

(Countersigned)

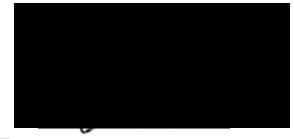

(Date) $\underline{31 / 08 / 2017}$

Corresponding author of paper: Anthony R. Carroll

(Countersigned)

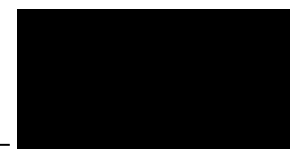

(Date) $\underline{31 / 08 / 2017}$

Principal supervisor: Anthony R. Carroll 


\subsection{Abstract}

Co-cultivation of different microbial strains can activate biosynthestic gene clusters that remain silent under standard laboratory fermentations involving mono-cultures. Therefore, the co-culturing technique has great potential for natural product drug discovery. However, innovative methods are still needed to evaluate the success of any co-cultured fermentation end product. Herein, the effective application of HSQC-TOCSY NMR spectra and subsequent PCoA for the detection of induced secondary metabolites in co-cultures between Streptomyces sp. (USC-16018), Micromonospora sp. (USC-16046), Nocardia sp. (USC-16096) and Staphylococcus aureus (ATCC 157293) is described.

\subsection{Introduction}

Rediscovery of already known compounds is a major issue in microbial natural product drug discovery. In recent years, progress has been made in developing more efficient dereplication approaches to minimise rediscovery. In parallel, whole genome sequencing of microorganisms has revealed their immense potential to produce secondary metabolites, yet the majority of biosynthetic genes remain silent under common laboratory culturing conditions. ${ }^{1-3}$ Therefore, increased research has focused on optimising culturing methods in order to activate the silent biosynthetic gene clusters. $^{4-6}$ The OSMAC (one-strain-many-compounds) strategy, which uses chemical and physical stressors in the media that trigger production of diverse secondary metabolites, has become widely implemented. ${ }^{7}$ Another effective approach to induce chemical diversity is to co-cultivate two of more microbial strains. ${ }^{8}$ In nature, microorganisms occur in dynamic communities and thus co-culture allows for interspecific interactions that resemble natural microbial ecosystems more closely and trigger the production of cryptic metabolites. ${ }^{9}$ Numerous examples of successful co-cultivation induced natural products, most often polyketides and non-ribosomal peptides, have been published and reviewed in the literature. ${ }^{6,10-14}$ Such examples highlight the potential of co-culturing for natural product drug discovery, however, effective methods for the detection of metabolite induction through co-cultivation are still necessary to evaluate their success.

Chemometric-profiling approaches have been used to assess the induction of microbial metabolites through co-cultures. ${ }^{12}$ Hyphenated methods such as LC-PDA or LC-MS are often used, but are dependent on the presence of chromophores or the ability of the natural products to ionise. NMR fingerprinting techniques have also been reported, by which the crude extract of mono- and cocultures were fractionated and ${ }^{1} \mathrm{H}$ NMR spectra of the pure cultures profiles were compared to those of the co-culture. ${ }^{10}$ The strengths of NMR profiling is that it provides insight into structural components of the extract, it is highly reproducible and provides universal detection. ${ }^{15}$ A technique 
using 2D HSQC-TOCSY NMR profiles to prioritise microbial strains that have higher potential to produce drug-like natural products was reported previously (Chapter 3). The advantage of using 2D NMR profiles is that chemical resolution is improved by spreading the structural information over two dimensions and consequently allows assessment of unfractionated crude extracts. Herein, the effectiveness of this approach to identify changes in the metabolite diversity induced through cocultivation was investigated.

\subsection{Results and Discussion}

For co-cultivation, four strains all originating from different species, namely Streptomyces sp. (USC-16018), Micromonospora sp. (USC-16046), Nocardia sp. (USC-16096) and Staphylococcus aureus (ATCC 157293), were chosen in order to assess a broader diversity of microbial strains. Liquid fermentation was favoured over solid agar cultures as mixed fermentations can more easily be upscaled, while on agar cultures the interactions between the two strains are generally highly localised and therefore compound isolation becomes more challenging as often low quantities of the compounds are produced. ${ }^{12}$ Mono- and co-cultures were fermented in liquid ASW-A media and after two weeks of incubation, the microbial cells were separated from the media and extracted in $\mathrm{MeOH}$. The liquid media phases were partitioned with EtOAc to extract the metabolites that were diffused into the media. The crude extracts of the mono- and co-cultures were profiled using 2D HSQCTOCSY NMR experiments. The advantage of this approach is that metabolite changes such as de novo production, and up- or down-regulation of metabolites in crude extracts can readily be detected in the spectra without fractionation of the crude extract. NMR further provides insights about the structural classes in the extract. This is particularly valuable for untargeted analysis, where a chemical profile is first established to assess chemical diversity within a sample and subsequent chemometric analysis can be used to identify uniqueness and similarities in the metabolome and reduce redundancy within the set of samples.

Visual inspection of the mono-culture profiles of the $\mathrm{MeOH}$ cell extracts depicted notable differences in microbial metabolomes at the genus level. The Micromonospora sp. (USC-16046) displayed resonances of high intensity associated with peptides that were not detected in the other three mono-cultures. Resonances associated with polyketide fragments were observed in the Nocardia sp. (USC-16096) mono-culture as well as numerous peptide-associated NMR signals and diverse aromatic resonances. The Staphylococcus species (ATCC 157293) profile appeared chemically less diverse but indicated the presence of unique polyketide signals and Streptomyces sp. (USC-16018) had the least diverse mycelia extract (Figure 1). In contrast, the EtOAc extract of the 
Streptomyces sp. mono-culture showed the most complex secondary metabolite resonances in the HSQC-TOCSY NMR spectrum with particular correlations from a proton at $\delta_{\mathrm{H}} 11.07 \mathrm{ppm}$ to carbons at $\delta_{\mathrm{C}} 111.9 \mathrm{ppm}$ and $\delta_{\mathrm{C}} 123.1 \mathrm{ppm}$ indicating the presence of a heteroaromatic compound (Figure 2). The other three EtOAc mono-culture extracts had almost identical HSQC-TOCY profiles (Figure 2).

Comparison of the HSQC-TOCSY spectra of the MeOH cellular mono- and co-culture extracts showed clearly that the co-culture between Micromonospora sp. (USC-16046) and S. aureus (ATCC 157293) had triggered de novo secondary metabolite production. Numerous aromatic resonances not observed in the mono-cultures of the two strains as well as several peptide-associated peaks could be identified in the HSQC-TOCSY NMR profile (Figure 1). Additional polyketide fragment resonances were observed in the co-culture of Streptomyces sp. (USC-16018) and Nocardia sp. (USC-16096) (Figure 1). The EtOAc extract of the co-culture between Streptomyces sp. (USC-16018) and S. aureus (ATCC 157293) indicated that heteroaromatic compounds had diffused into the culture media (correlations of $\delta_{\mathrm{H}} 8.08$ and 8.16 to $\delta_{\mathrm{C}} 152.8$ ) (Figure 2). 


\begin{tabular}{|c|c|c|c|c|}
\hline Strain & Streptomyces sp. USC-16018 & Micromonospora sp. USC-16046 & Nocardia sp. USC-16096 & S. aureus ATCC 157293 \\
\hline 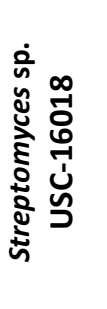 & 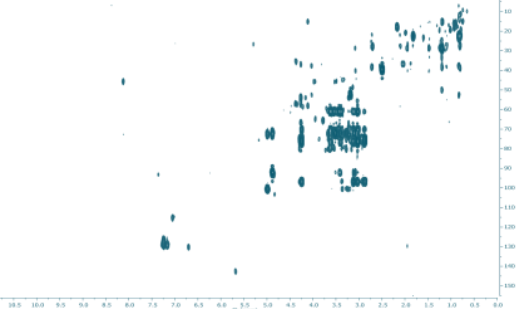 & 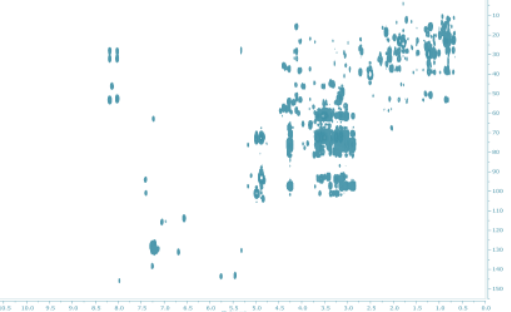 & 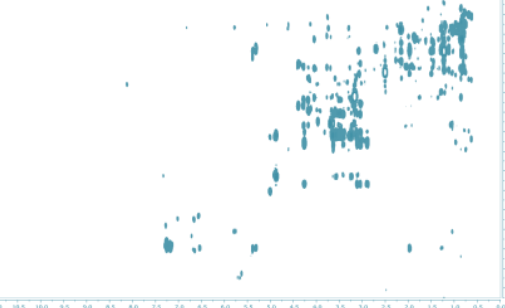 & 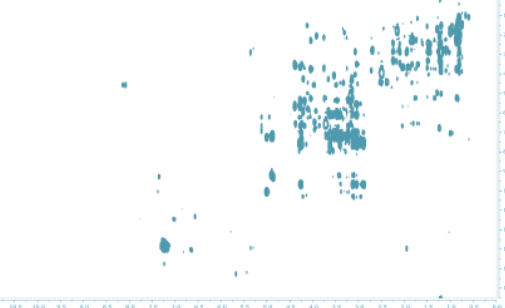 \\
\hline 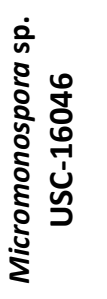 & & 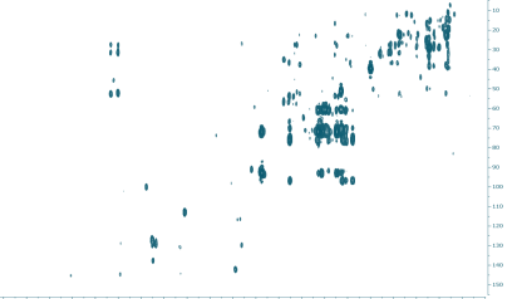 & 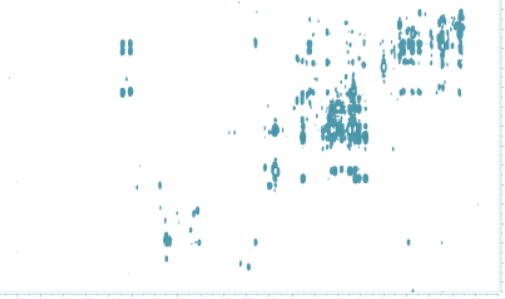 & 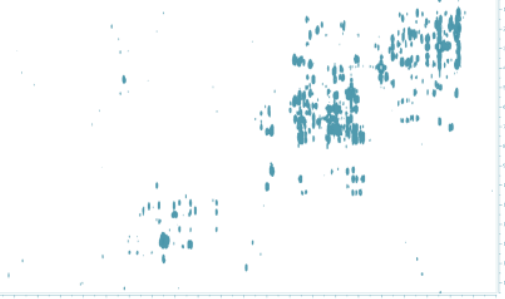 \\
\hline 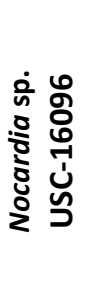 & & & 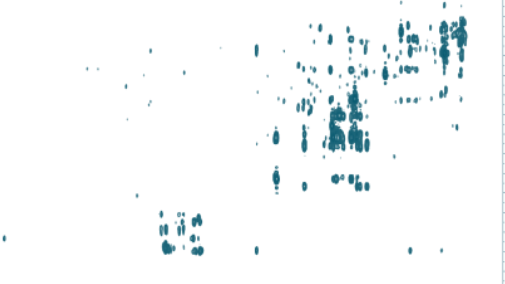 & 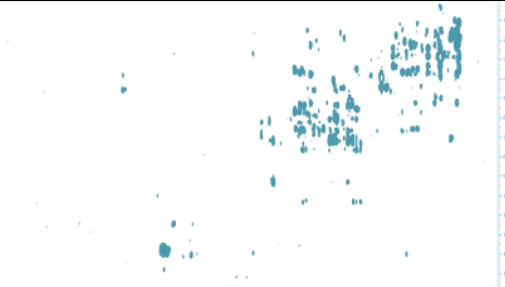 \\
\hline 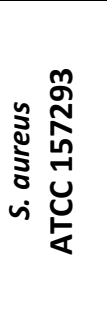 & & & & 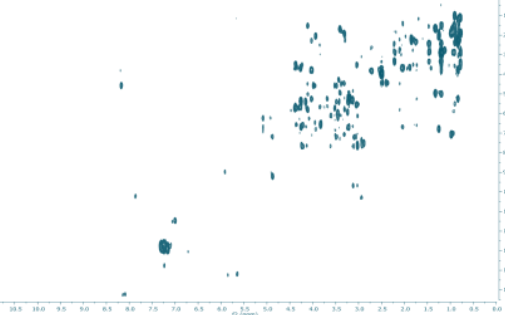 \\
\hline
\end{tabular}

Figure 1. HSQC-TOCSY NMR profile matrix of mono- and co-cultures of MeOH mycelia extracts from four different microbial strains (dark teal $=\mathrm{MeOH}$ extracts of mono-cultures, light teal $=\mathrm{MeOH}$ extracts of co-cultures $)$ 


\begin{tabular}{|c|c|c|c|c|}
\hline Strain & Streptomyces sp. USC-16018 & Micromonospora sp. USC-16046 & Nocardia sp. USC-16096 & S. aureus ATCC 157293 \\
\hline 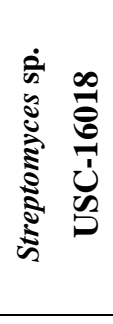 & $1 \quad$ & & & \\
\hline 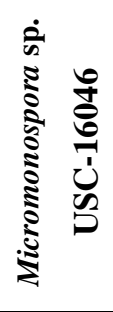 & 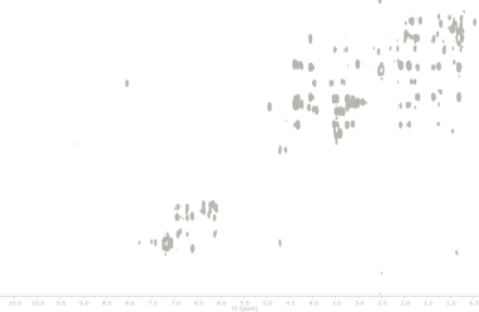 & 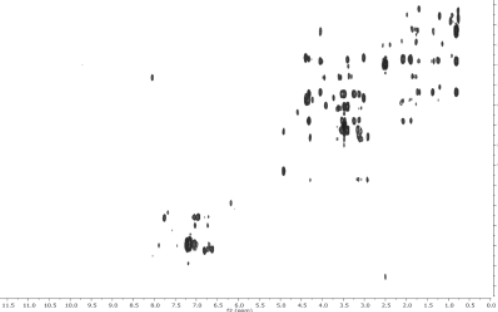 & & \\
\hline 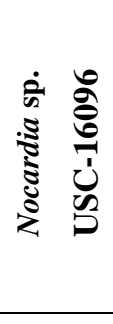 & , m? & $\therefore$ & (") & \\
\hline 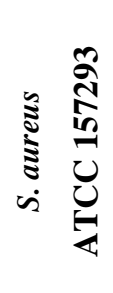 & $\therefore$ and & 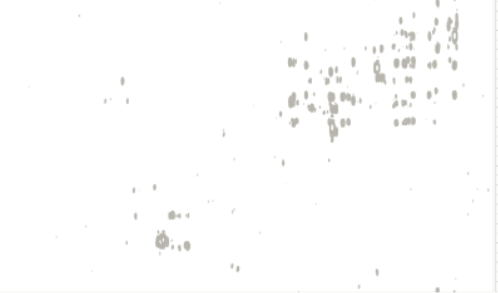 & $\sum_{0,1}$ & 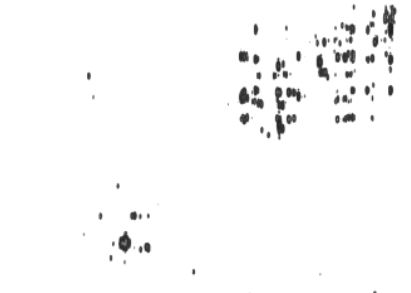 \\
\hline
\end{tabular}

Figure 2. HSQC-TOCSY NMR profile matrix of mono- and co-cultures of liquid media extracts from four different microbial strains (black $=$ EtOAc extracts of mono-cultures, grey $=$ EtOAc extracts of co-cultures) 
A chemometric metabolomics approach using multivariate analysis was implemented in order to dereplicate the dataset and identify significant correlations and differences in the secondary metabolomes. As described in the strain selection approach (Chapter 3), peaks were picked from the HSQC-TOCSY NMR spectra regions of interest that were associated with polyketide, peptide, double bond, aromatic and heteroaromatic structure fragments. After removal of resonances found in the media control, this resulted in a total of 253 HSQC-TOCSY cross-peaks associated with secondary metabolites. Peaks between the samples were aligned using an in-house Excel macro. Dice similarities were calculated and the newly generated correlation matrix was used for Principal Coordinate Analysis (PCoA, Figure 3). The first two principal coordinates (F1/F2) were able to explain $46.6 \%$ of the variation within the dataset.

Clear separation of the EtOAc liquid media extracts and the $\mathrm{MeOH}$ cell mycelium extracts could be observed (Figure 3). Furthermore, the mono-cultures of Streptomyces sp. (USC-16018) (S) and its co-cultures with Micromonospora sp. (USC-16046) and Nocardia sp. (USC-16096) (SM and SN) clustered away from the remaining EtOAc culture extracts. Visual inspection of their HSQC-TOCSY NMR profiles revealed that they were almost identical and dominated by Streptomyces sp. (USC-16018) metabolites. The remaining liquid media extracts grouped closely together in the PCoA plot (Figure 3); therefore, Streptomyces sp. (USC-16018) metabolites in the co-culture with S. aureus (ATCC 157293) should be suppressed. These observations were further validated through comparison of the HSQC-TOCSY NMR profiles (Figure 3).

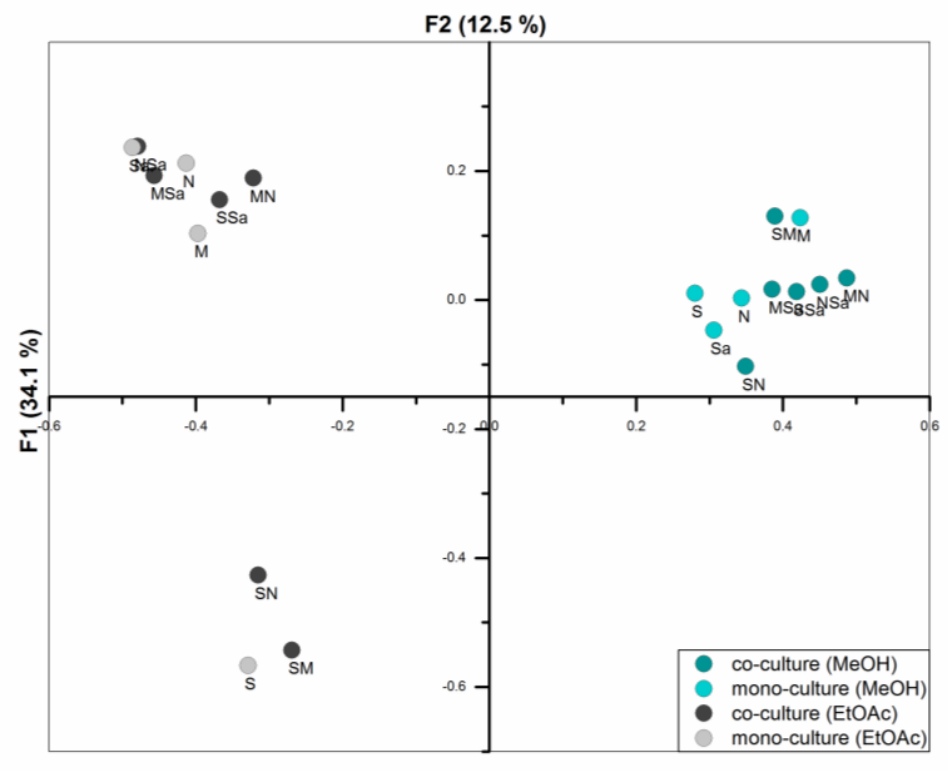

Figure 3. PCoA plot showing differences between MeOH and EtOAc extracts of mono- and cocultures ( $\mathrm{S}=$ Streptomyces $\mathrm{sp} ., \mathrm{M}=$ Micromonospora $\mathrm{sp} ., \mathrm{N}=$ Nocardia $\mathrm{sp}$., $\mathrm{Sa}=$ Staphylococcus aureus) 
The differences in secondary metabolites of the $\mathrm{MeOH}$ extracts were not as obvious to derive from the PCoA plot; therefore, separate PCoA plots for $\mathrm{MeOH}$ and EtOAc extracted cultures were generated (Figure 4). Based on the position of the mono-cultures compared to the co-culture it could be determined which of the two strains was the more dominant in the culture (Figure 4a). The coculture of Micromonospora sp. (USC-16046) and S. aureus (ATCC 157293) (MSa) was distinct to both mono-cultures reiterating the uniqueness of this co-culture previously observed in the HSQCTOCSY profile.
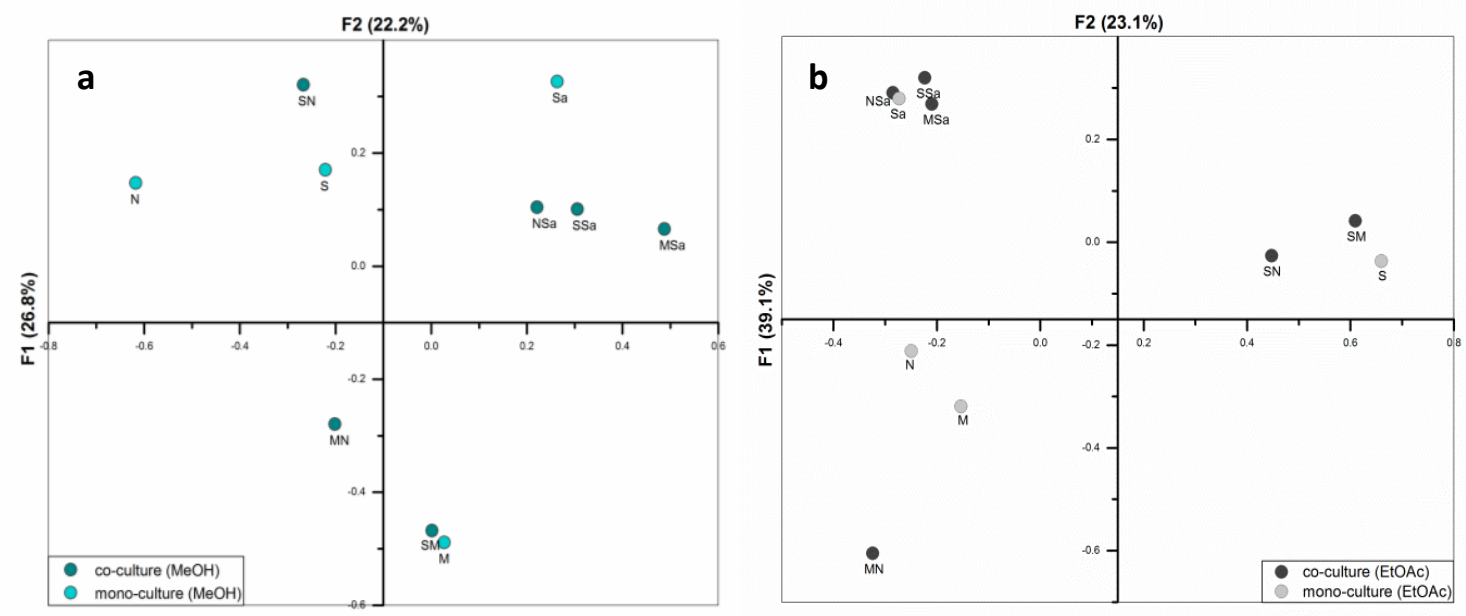

Figure 4. PCoA plot showing differences between (a) $\mathrm{MeOH}$ mycelia extracts and (b) EtOAc liquid culture media extracts $(\mathrm{S}=$ Streptomyces $\mathrm{sp} ., \mathrm{M}=$ Micromonospora $\mathrm{sp} ., \mathrm{N}=$ Nocardia $\mathrm{sp} ., \mathrm{Sa}=$ Staphylococcus aureus)

The HSQC-TOCSY NMR profiling approach proved to be very useful for the assessment of cocultures. Not only did the profiles provide insight about de novo induction of secondary metabolites but in some cases also their suppression. The Micromonospora sp. (USC-16046) peptide resonances, which were dominant in all other $\mathrm{MeOH}$ co-cultures with the species, were highly suppressed in the co-culture with S. aureus (ATCC 157293), while aromatic resonances were significantly enhanced. Furthermore, it was clearly evident that the microorganisms diffuse secondary metabolites into the media that significantly differ from the metabolites found in their mycelia. Therefore, it is valuable to consider a range of extraction methods and solvents to obtain a complete spectrum of secondary metabolites in the culture.

Observations about the success and what type of structural fragments were found in the extracts could be made with minimal sample preparation in very little time as these 2D NMR experiments took only half an hour to acquire. The integration of chemometric analysis can be a useful guide to detect unique samples. Especially, in larger datasets where sometimes similarities or differences in the 
NMR spectra cannot be identified solely by visual inspection. In a co-culture setting, chemometric metabolomics analysis is more suitable than in the strain selection approach (Chapter 2) where the samples were extremely diverse. All co-culture will be related to the mono-cultures of the strains and thus better "fit" in the statistical multivariate analysis.

Only few examples have been reported that utilise NMR profiles for the assessment of cocultures. ${ }^{10,16-18} \mathrm{Wu}$ and co-workers, implemented a ${ }^{1} \mathrm{H}$-NMR-based metabolomics approach to study metabolomic changes in a Streptomyces-fungus co-culture and found significant differences between mono- and co-culture extracts, but only in combination with UHPLC-TOF-MS they were able to identify the compound classes. ${ }^{17}$ The HSQC-TOCSY profiling approach here presented is the first account where 2D NMR profiling was used on co-cultivations. Due to the increased chemical shift resolution through spreading of the detected signals across two planes in 2D NMR spectra, assessment of unfractionated crude extracts was permitted and immediately revealed the structure type of the induced secondary metabolites.

In the future, it will be interesting to pursue large-scale fermentation of the co-culture between $S$. aureus (ATCC-157293) and either Micromonospora sp. (USC-16046) and or Streptomyces sp. (USC16018 ) in order to identify the co-culture induced de novo produced secondary metabolites.

\subsection{Experimental section}

\subsubsection{Microbial fermentations}

Streptomyces sp. (USC-16018), Micromonospora sp. (USC-16046), Nocardia sp. (USC-16096) and were isolated from the Australian ascidian Symplegma rubra (Chapter 2). Staphylococcus aureus (ATCC-157293) strain was obtained from Mr Joshua Hayton (Griffith University, Gold Coast). Each strain obtained from $20 \%$ glycerol stocks was fermented in $15 \mathrm{~mL}$ Eppendorf tubes containing $4 \mathrm{~mL}$ of brain heart infusion broth (Thermo-Fischer) and incubated at $28{ }^{\circ} \mathrm{C}$ and $200 \mathrm{rpm}$ for 4 days. Subsequently, $0.5 \mathrm{~mL}$ of each culture were individually transferred to $50 \mathrm{~mL}$ Cornig MiniBioreactors with $20 \mathrm{~mL}$ ASW-A (20.0 g soluble starch, $10.0 \mathrm{~g}$ glucose, $5.0 \mathrm{~g}$ peptone, $5.0 \mathrm{~g}$ yeast extract, $5.0 \mathrm{~g}$ $\mathrm{CaCO} 3$ per litre of 186 artificial seawater) and kept at $28{ }^{\circ} \mathrm{C}$ and rotation (200 rpm) again for 4 days. For the co-cultivation experiment, $0.8 \mathrm{~mL}$ of each strain was separately inoculated with $0.8 \mathrm{~mL}$ of each of opponent strain in $100 \mathrm{~mL}$ conical flask containing $60 \mathrm{~mL}$ ASW-A and kept at rotation $(150 \mathrm{rpm})$ at $28^{\circ} \mathrm{C}$ for 2 weeks. In parallel, one mono-culture of each of the four microbial strains was also incubated under the same conditions as controls. 


\subsubsection{Preparation of microbial extracts}

After fermentation, the cellular mycelium was separated from the culture media by filtration through refillable cellulose tea bags. The harvested cell mycelia were washed with deionised water and subsequently exhaustively extracted in $\mathrm{MeOH}$. The liquid media phases were transferred into a $250 \mathrm{~mL}$ separation funnels and twice extracted with $60 \mathrm{~mL}$ EtOAc. All extracts were dried under vacuum (GeneVac Technologies HP-12), resuspended in a small portion of $\mathrm{MeOH}$, then filtered using a $0.22 \mu \mathrm{m}$-pore-size filter and subsequently dried. Yields per culture varied between $4-72 \mathrm{mg}$.

For NMR spectroscopy, the unfractionated extracts, as well as an ASW-A media blank, were suspended in $200 \mu \mathrm{L}$ DMSO- $d_{6}$ and transferred to $3 \mathrm{~mm}$ NMR tubes. The ${ }^{1} \mathrm{H}$ NMR data was acquired under following parameters: number of scans $16,{ }^{1} \mathrm{H} 90^{\circ}$ pulse $8.4 \mu \mathrm{sec}$, interscan relaxation delay $0.8 \mathrm{sec}$, spectral width $20.0294 \mathrm{ppm}$, transmitter centre $6.175 \mathrm{ppm}, \mathrm{O} 1$ 4940.62. HSQC-TOCSY spectra were obtained at: number of scans $16,{ }^{1} \mathrm{H} 90^{\circ}$ pulse $8.4 \mu \mathrm{sec}$, interscan relaxation delay $0.8 \mathrm{sec}$, increments 128, spectral width $16.0235 \mathrm{ppm}$, transmitter centre $6.000 \mathrm{ppm}, \mathrm{O} 14800.6 \mathrm{~Hz}, \mathrm{~F} 1$ spectral width $170 \mathrm{ppm}, \mathrm{F} 1$ transmitter centre $80.000 \mathrm{ppm}$, spinlock mixing time $0.06 \mathrm{sec}$.

In MestReNova 11.0, proton spectra were corrected for phase and baseline and referenced to the DMSO solvent peak (2.50 ppm). For processing of HSQC-TOCSY spectra, t2 noise was reduced, apodization was set to $0.00^{\circ}$ sine bell, $10.00 \mathrm{~Hz}$ Gaussian and $50.0 \%$ Sine Bell II and Sine Square II in the $\mathrm{F} 2$ dimension and to $90^{\circ}$ sine bell in F1; spectra were again referenced to the DMSO solvent peak $\left(\delta_{\mathrm{H}} 2.50 \mathrm{ppm}\right.$ and $\left.\delta_{\mathrm{C}} 39.51 \mathrm{ppm}\right)$. Cross peaks were picked by zooming into the regions of interest setting peak picking to manual threshold with the peak picking options set to 4.00 sensitivity and maximum number of peaks 1000 and dragging the cursor over the spectra.; for polyketide specific peaks from 0.00 to $2.00 \mathrm{ppm}$ in the proton dimension and from 60.0 to $160.0 \mathrm{ppm}$ in the carbon dimension, for peptides from $6.00 \mathrm{ppm}-14.00 \mathrm{ppm}(\mathrm{F} 2)$ and 10.0 to $100.0 \mathrm{ppm}$ (F1) and heteroaromatics and double bonds with electron withdrawing atoms between $5.00-14.00 \mathrm{ppm}(\mathrm{F} 1)$ and 135 - $160 \mathrm{ppm}$ (F2). If several spectra were selected, MestReNova allowed peak picking to be performed in parallel on all selected spectra. All 2D peaks were exported as text files and peak data was tabulated in Excel. Using an Excel macro, peaks between samples were aligned allowing for an error threshold of $0.04 \mathrm{ppm}$ for proton shifts and $0.4 \mathrm{ppm}$ for carbon chemical shifts. A Dice similarity matrix was then established and used for Principal Coordinate Analysis (PCoA), which was visualised in OriginPro. 


\subsection{References}

(1) Jensen, P. R.; Chavarria, K. L.; Fenical, W.; Moore, B. S.; Ziemert, N. J. Ind. Microbiol. Biotechnol. 2014, 41, 203.

(2) Osbourn, A. Trends Genet. 2010, 26, 449.

(3) Udwary, D. W.; Zeigler, L.; Asolkar, R. N.; Singan, V.; Lapidus, A.; Fenical, W.; Jensen, P. R.; Moore, B. S. Proc. Natl. Acad. Sci. U. S. A. 2007, 104, 10376.

(4) Zerikly, M.; Challis, G. L. ChemBioChem 2009, 10, 625.

(5) Abdelmohsen, U. R.; Grkovic, T.; Balasubramanian, S.; Kamel, M. S.; Quinn, R. J.; Hentschel, U. Biotechnol. Adv. 2015, 33, 798.

(6) Scherlach, K.; Hertweck, C. Org. Biomol. Chem. 2009, 7, 1753.

(7) Bode, H. B.; Bethe, B.; Höfs, R.; Zeeck, A. ChemBioChem 2002, 3, 619.

(8) Marmann, A.; Aly, A. H.; Lin, W.; Wang, B.; Proksch, P. Mar. Drugs 2014, 12, 1043.

(9) De Roy, K.; Marzorati, M.; Van den Abbeele, P.; Van de Wiele, T.; Boon, N. Environ. Microbiol. 2014, 16, 1472.

(10) Dashti, Y.; Grkovic, T.; Abdelmohsen, U. R.; Hentschel, U.; Quinn, R. J. Mar. Drugs 2014, 12, 3046.

(11) Cueto, M.; Jensen, P. R.; Kauffman, C.; Fenical, W.; Lobkovsky, E.; Clardy, J. J. Nat. Prod. 2001, 64, 1444.

(12) Bertrand, S.; Bohni, N.; Schnee, S.; Schumpp, O.; Gindro, K.; Wolfender, J.-L. Biotechnol. Adv. 2014, 32, 1180 .

(13) Tashiro, Y.; Yawata, Y.; Toyofuku, M.; Uchiyama, H.; Nomura, N. Microbes Environ. 2013, $28,13$.

(14) Gonzalez, D. J.; Haste, N. M.; Hollands, A.; Fleming, T. C.; Hamby, M.; Pogliano, K.; Nizet, V.; Dorrestein, P. C. Microbiology 2011, 157, 2485.

(15) Kurita, K. L.; Linington, R. G. J. Nat. Prod. 2015, 78, 587.

(16) Cheng, Y. F.; Jin, W.; Mao, S. Y.; Zhu, W.-Y. Asian-Australas. J. Anim. Sci. 2013, 26, 1416.

(17) Wu, C.; Zacchetti, B.; Ram, A. F.; Van Wezel, G. P.; Claessen, D.; Choi, Y. H. Sci. Rep. 2015, 5 .

(18) Bertrand, S.; Azzollini, A.; Schumpp, O.; Bohni, N.; Schrenzel, J.; Monod, M.; Gindro, K.; Wolfender, J.-L. Mol. BioSyst. 2014, 10, 2289. 


\section{SECTION 3}

\section{Marine actinomycetes as a source of new anti- plasmodial compounds}

Malaria is a highly infectious and potentially deadly disease caused by parasites from the genus Plasmodium. Due to the evolution of drug resistance in the parasite, it is still of high importance to find new drugs to treat this disease. This section focuses chemical investigation on marine actinomycetes as a source for anti-plasmodial compounds. The application of the new HSQC-TOCSY NMR profiling approach presented in Chapter 3 indicated that Streptomyces sp. (USC-16018) contains a wealth of polyketide associated NMR signals in its crude extract, which also exhibited $97 \%$ inhibition of $P$. falciparum at a concentration of $0.2 \mu \mathrm{g} / \mathrm{mL}$. Chapter 5 of this thesis documents the isolation and structure elucidation of a new polyketide with weak anti-plasmodial activity, as well as isolation of five known natural products.

In Chapter 6, isolation of another new and moderately anti-plasmodial natural product from Streptomyces sp. (USC-636) is described. This actinomycete strain was previously isolated from marine sediment by my supervisor D. Ipek Kurtböke. I pursued chemical investigations of this isolate in the early stages of this $\mathrm{PhD}$ project and it benefited as a proof of concept to determine the potential of actinomycetes as producers of anti-plasmodial molecules. 


\section{CHAPTER 5 - HSQC-TOCSY profiling directed discovery of anti-plasmodial polyketide compounds in marine Streptomyces species (USC-16018)}

\section{STATEMENT OF CONTRIBUTION TO CO-AUTHORED PAPER IN PREPARATION}

This chapter is a co-authored paper in preparation for submission. The bibliographic details of the co-authored paper, including all authors, are:

Buedenbender, L., Robertson, L.R., Lucantoni , L., Kurtböke, D.I., Carroll, A.R., HSQCTOCSY profiling directed discovery of anti-plasmodial polyketide compounds in marine Streptomyces species (USC-16018), in preparation

The author this thesis, L. Buedenbender fermented the microbial strain, isolated, and elucidated the natural products and prepared the manuscript. All of the theoretical calculations for the ECD spectra presented were the work of Luke P. Robertson. Dr Leonardo Lucantoni, from "Discovery Biology" of the Griffith Institute for Drug Discovery performed biological activity testing. The co-authors D. I. Kurtböke and A. R. Carroll are the supervisors of the PhD project and provided advice on the research design and feedback.

(Signed)

(Date) $\underline{01 / 09 / 2017}$

Larissa Buedenbender

(Countersigned)

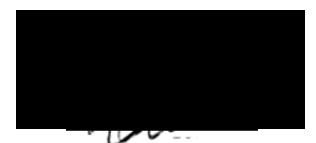

(Date) $\underline{31 / 08 / 2017}$

Corresponding author of paper: Anthony R. Carroll

(Countersigned)

(Date) $31 / 08 / 2017$

Principle supervisor: Anthony R. Carroll 


\subsection{Abstract}

Chemical investigations of the EtOAc extract of the ascidian-derived Streptomyces sp. (USC-16018) yielded a new ansamycin polyketide, herbimycin G (1) another known macrocyclic polyketide, elaiophylin (2) and four known diketopiperazines $(3-6)$. The structures of the compounds were elucidated based on 1D/2D NMR and MS data. The absolute configuration of $\mathbf{1}$ was established by comparison of experimental and predicted ECD spectra, calculated using TDDFT. Anti-plasmodial activities were tested for the natural products against chloroquine sensitive (3D7) and chloroquine resistant (Dd2) Plasmodium falciparum strains; the two polyketides $(\mathbf{1}$ - 2) caused significant inhibition of $>75 \%$ against both parasite strains and while 2 was highly cytotoxic, the new compound (1) showed no cytotoxicity and good water solubility.

\subsection{Introduction}

Malaria is a vector borne disease, induced by protozoan parasites of the genus Plasmodium, and has caused millions of deaths worldwide. ${ }^{1}$ The life cycle of the malaria parasite involves two hosts. The disease is contracted when an Anopheles mosquito feeds on human blood and simultaneously injects sporozoites into the blood stream. The sporozoites are then carried to the liver, where they asexually reproduce to give rise to thousands of merozoites that spread through the body via the blood stream. ${ }^{2,3}$ At this intraerythrocytic stage, the disease manifests and the human host experiences strong fevers and chills. ${ }^{4}$ Within the red blood cells, merozoites develop into the early trophozoites also known as the ring form. The mature trophozoites asexually multiply forming schitzonts to finally produce male and female gametocytes. The gametocytes are transmitted back to a mosquito as it takes a blood meal and there they fuse to form oocysts, which divide into sporozoites. These are then transferred to the mosquito's salivary glands and the life cycle of the parasite begins again once the mosquito feeds on a new human's blood. Drug resistance against the currently available malaria treatments is a major concern and therefore new drugs with novel targets are urgently needed.

When the malaria parasite changes from mosquito to human hosts, it experiences immense heat stress and specific heat shock proteins are highly expressed in Plasmodium species, particularly during the intraerythrocytic stage of their life cycle. ${ }^{5}$ Recently, anti-plasmodial drug development has been focused on heat shock protein 90 (Hsp90) inhibitors. Benzoquinone ansamycin compounds, such as geldanamycins and herbimycins, produced by Streptomyces sp. strains, are known to exhibit specific inhibition of Hsp90. In vitro studies have found that geldanamycin inhibits the growth of chloroquine-sensitive and chloroquine-resistant 
Plasmodium falciparum strains ( $\mathrm{IC}_{50} 20 \mathrm{nM}$ ). ${ }^{6}$ Other geldanamycin derivatives were reported to be effective against Plasmodium yoelii in mouse models when injected in repeated doses of $300 \mathrm{nM} /$ mouse and treated mice appeared to develop immunity against reinfection. ${ }^{7}$ Thus, ansamycin analogues appear to be good candidates for anti-plasmodial drugs. Due to the low water solubility and high liver toxicity of geldanamycin, it is of great interest to find new water soluble and non-cytotoxic Hsp90 inhibitors to treat malaria infections.

Actinomycetes are the main class of bacteria known to produce ansamycins. In order to increase structural diversity, the presented study focused microbial isolations on underexplored ecological niches and 120 actinomycetes from Australian ascidian species were isolated. A new approach utilising two-dimensional HSQC-TOCSY NMR profiles and biological activity data was previously described (Chapter 3). This allowed targeting of polyketide-producing strains based on identified regions of interest in the NMR spectrum that corresponded to correlations from upfield methyl proton resonances $\left(\delta_{\mathrm{H}} 0.50-2.00\right)$ into carbon resonances between $\delta_{\mathrm{C}} 60$ 80 ppm (Appendix III: Figure IIIA), which were associated with polyketide-type compounds. Based on this approach Streptomyces sp. strain USC-16018 was selected because its crude extract HSQC-TOCSY NMR profile indicated the presence of several polyketide containing compounds and the extracts also exhibited $97 \%$ inhibition of Plasmodium falciparum at $0.2 \mu \mathrm{g} / \mathrm{mL}$. The strain was fermented on a larger scale and purification of the EtOAc extract derived from this culture resulted in the isolation of two anti-plasmodial polyketides, herbimycin G (1), a new geldanamycin analogue, and the known antibiotic elaiophylin (2), as well as four known diketopiperazines (3 - 6).

\subsection{Results and discussion}

Fermentations were scaled up on 55 culture plates for two solid media, OMA and GST, which after extraction with EtOAc yielded $1.6 \mathrm{~g}$ and $0.8 \mathrm{~g}$ of crude extract respectively. The crude extracts were separated on a reversed-phase $\mathrm{C}_{18}$ HPLC column and 60 fractions were collected and analysed by ${ }^{1} \mathrm{H}$ NMR. NMR-guided isolation led to the identification of two polyketides (1 and $\mathbf{2}$ ) and four diketopiperazine compounds (3-6).

Herbimycin G (1) was isolated as a dark brown solid with a UV maximum at $245 \mathrm{~nm}$. A sodium adduct ion at $m / z 602.3111[\mathrm{M}+\mathrm{Na}]^{+}$obtained from HRESI-QTOF-MS together with ${ }^{1} \mathrm{H}$ and ${ }^{13} \mathrm{C}$ NMR data suggested that $\mathbf{1}$ had a molecular formula of $\mathrm{C}_{30} \mathrm{H}_{46} \mathrm{~N}_{2} \mathrm{O}_{9}$. The ${ }^{1} \mathrm{H}$ NMR spectrum of 1 in DMSO- $d_{6}$ had resonances that could be assigned to an amide proton at $\delta_{\mathrm{H}} 8.02$ $(\mathrm{d}, J=9.4 \mathrm{~Hz})$, five olefinic proton resonances at $\delta_{\mathrm{H}} 7.13(\mathrm{dd}, J=12.2 \mathrm{~Hz}, 1.2 \mathrm{~Hz}), \delta_{\mathrm{H}} 6.65$ $(\mathrm{ddd}, J=, 3.3 \mathrm{~Hz}, 1.9 \mathrm{~Hz}, 1.4 \mathrm{~Hz}), \delta_{\mathrm{H}} 6.48(\mathrm{ddd}, J=12.1 \mathrm{~Hz}, 10.9 \mathrm{~Hz}, 1.4 \mathrm{~Hz}), \delta_{\mathrm{H}} 5.65(\mathrm{dd}$, 
$J=10.9 \mathrm{~Hz}, 7.9 \mathrm{~Hz})$ and $\delta_{\mathrm{H}} 5.20(\mathrm{~d}, J=7.7 \mathrm{~Hz})$, and an oxygenated methine at $\delta_{\mathrm{H}} 4.68$ (dddd, $J=10.6, \mathrm{~Hz} 5.1 \mathrm{~Hz}, 4.7 \mathrm{~Hz}, 2.5 \mathrm{~Hz})$ that correlated to a hydroxyl group at $\delta_{\mathrm{H}} 5.56$ (d, $J=5.1$ $\mathrm{Hz})$ in the COSY spectrum. A further five oxygenated methines were observed at $\delta_{\mathrm{H}} 4.56(\mathrm{dd}$, $J=7.9 \mathrm{~Hz}, 1.4 \mathrm{~Hz}), \delta_{\mathrm{H}} 5.16(\mathrm{~s}), \delta_{\mathrm{H}} 3.15, \delta 3.33$ and $\delta_{\mathrm{H}} 4.15(\mathrm{~d}, \mathrm{~J}=1.4 \mathrm{~Hz})$, and four methoxyresonances occurred between $\delta_{\mathrm{H}} 3.13-3.35 \mathrm{ppm}$. The ${ }^{1} \mathrm{H}$ NMR spectrum contained an additional three methines at $\delta_{\mathrm{H}} 4.55, \delta_{\mathrm{H}} 2.45, \delta_{\mathrm{H}} 1.51$, two methylene pairs $\delta_{\mathrm{H}} 1.55$ and $\delta_{\mathrm{H}} 1.41$ as well as $\delta_{\mathrm{H}} 1.96$ and $\delta_{\mathrm{H}} 2.26$, and four methyl signals $\delta_{\mathrm{H}} 1.84(\mathrm{~d}, J=1.2 \mathrm{~Hz}), \delta_{\mathrm{H}} 1.62(\mathrm{~s})$, $\delta_{\mathrm{H}} 0.94(\mathrm{~d}, J=6.7 \mathrm{~Hz})$ and $\delta_{\mathrm{H}} 0.69(\mathrm{~d}, J=6.7 \mathrm{~Hz})$. The ${ }^{13} \mathrm{C}$ and HSQC NMR data showed 30 carbon resonances (Table 1).

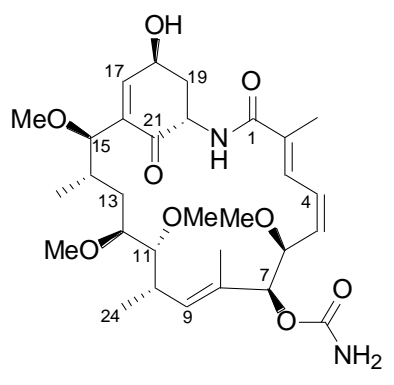

1

COSY and HSQC analysis, as well as coupling constants revealed that three of the five olefinic protons belonged to the same spin system. The olefinic methine at $\mathrm{H}-3\left(\delta_{\mathrm{H}} 7.13 / \delta_{\mathrm{C}} 127.5\right)$ showed large coupling $(12.2 \mathrm{~Hz})$ to $\mathrm{H}-4\left(\delta_{\mathrm{H}} 6.48 / \delta_{\mathrm{C}} 126.4\right)$, which also showed cis coupling $(10.8 \mathrm{~Hz})$ to $\mathrm{H}-5\left(\delta_{\mathrm{H}} 5.65 / \delta_{\mathrm{C}} 135.4\right)$. Further COSY correlations from H-5 to an oxygenated proton between $4.55-4.57 \mathrm{ppm}$ were observed, however due to overlap of two protons in this shift range assignment through COSY correlations was ambiguous. Therefore, a TOCSY experiment was acquired and careful inspection revealed that the proton that resonated further downfield $\left(\delta_{\mathrm{H}} 4.57 / \delta_{\mathrm{C}} 78.1\right)$ was, in fact, the proton alpha to $\mathrm{H}-5$ and it also showed a weak TOCSY correlation to a proton resonating at $\delta_{\mathrm{H}} 5.16 / \delta_{\mathrm{C}} 78.0(\mathrm{H}-7)$ that was not seen in the COSY spectrum. A second spin system was elucidated based on COSY correlations between the olefinic proton $\mathrm{H}-9\left(\delta_{\mathrm{H}} 5.20 / \delta_{\mathrm{C}} 129.4\right)$ and the methine $\mathrm{H}-10\left(\delta_{\mathrm{H}} 2.48 / \delta_{\mathrm{C}} 33.5\right)$. The methine $\mathrm{H}-10$, further showed a strong COSY correlation to the methyl attached to C-24 $\left(\delta_{\mathrm{H}} 0.94 / \delta_{\mathrm{C}} 17.2\right)$ as well as the oxygenated methine $\mathrm{H}-11\left(\delta_{\mathrm{H}} 3.15 / \delta_{\mathrm{C}} 83.8\right)$. COSY and HSQC analysis revealed a third isolated spin system, 1-hydroxy-3-amino-propyl, which included the exchangeable proton $\left(\delta_{\mathrm{H}} 8.02\right)$. TOCSY correlations from the olefinic proton resonating at $\delta_{\mathrm{H}}$ $6.65 / \delta_{\mathrm{C}} 149.9$ to all protons of the third spin system implied that the olefinic proton was part of the system. Lastly, COSY correlations from the methyl protons resonating at $\delta_{\mathrm{H}} 0.69 / \delta_{\mathrm{C}} 13.9$ to $\mathrm{H}-14\left(\delta_{\mathrm{H}} 1.51 / \delta_{\mathrm{C}} 36.6\right)$ and then to the methylene at C-13 $\left(\delta_{\mathrm{H}} 1.41\right.$ and $\left.\delta_{\mathrm{H}} 1.55 / \delta_{\mathrm{C}} 33.8\right)$, which also correlated to H-12 $\left(\delta_{\mathrm{H}} 3.33 / \delta_{\mathrm{C}} 82.3\right)$ revealed a fourth spin system. Remaining were two methyls at $\delta_{\mathrm{H}} 1.84 / \delta_{\mathrm{C}} 12.9$ and $\delta_{\mathrm{H}} 1.62 / \delta_{\mathrm{C}} 14.2$, as well as an oxygenated methine with a resonance at $\delta_{\mathrm{H}} 4.15 / \delta_{\mathrm{C}} 77.6$, which all did not show any COSY nor TOCSY correlations. 
Table 1. NMR data for herbimycin G (1) (800 MHz in DMSO- $d_{6}, \delta$ in ppm)

\begin{tabular}{|c|c|c|c|c|c|}
\hline Position & $\boldsymbol{\delta}_{\mathrm{C}}$ & $\boldsymbol{\delta}_{\mathbf{H}}$ (multiplicity, $J$ in $\mathrm{Hz}$ ) & COSY & НМBC & ROESY \\
\hline $\mathrm{NH}$ & & $8.02(\mathrm{~d}, 9.4)$ & 20 & 1,20 & $3,19 \mathrm{a}$ \\
\hline 1 & 171.5 & & & & \\
\hline 2 & 133.6 & & & & \\
\hline 3 & 127.5 & $7.13(\mathrm{dd}, 12.2,1.2)$ & 4 & $1,3,5,22$ & \\
\hline 4 & 126.4 & $6.48(\mathrm{ddd}, 12.2,10.9,1.4)$ & 3,5 & 2,6 & \\
\hline 5 & 135.4 & $5.65(\mathrm{dd}, 10.9,7.9)$ & 4,6 & 4 & \\
\hline 6 & 78.1 & $4.56(\mathrm{dd}, 7.9,1.4)$ & 5,7 & 5 & 7 \\
\hline $6-\mathrm{OCH}_{3}$ & 56.5 & $3.20(\mathrm{~s})$ & & & 6,24 \\
\hline 7 & 78.0 & $5.16(\mathrm{~s})$ & 6 & 8 & \\
\hline 7-OCONH${ }_{2}$ & 156.5 & $6.66,6.30$ & & & \\
\hline 8 & 133.0 & & & & \\
\hline 9 & 129.4 & $5.20(\mathrm{~d}, 7.7)$ & 10 & 23 & \\
\hline 10 & 33.5 & $2.45, \mathrm{~m}$ & $9,11,24$ & 8,11 & \\
\hline 11 & 83.8 & $3.15, \mathrm{~m}$ & 10,12 & $12-\mathrm{OCH}_{3}, 13$ & \\
\hline $11-\mathrm{OCH}_{3}$ & 59.8 & $3.35(\mathrm{~s})$ & & 11 & 12,24 \\
\hline 12 & 82.3 & $3.33, \mathrm{~m}$ & 11,13 & & $11-\mathrm{OCH}_{3}$ \\
\hline $12-\mathrm{OCH}_{3}$ & 56.5 & $3.19(\mathrm{~s})$ & & 12 & $13 \mathrm{a}$ \\
\hline $13 \mathrm{a}$ & 33.8 & 1.41 (dddd, $14.2,9.7,3.4,3.4)$ & & & 12,14 \\
\hline $13 b$ & & $1.55, \mathrm{~m}$ & & & \\
\hline 14 & 36.6 & $1.51, \mathrm{~m}$ & $13,15,25$ & & \\
\hline 15 & 77.6 & $4.15(\mathrm{~d}, 1.4)$ & 14 & $13,15-\mathrm{OCH}_{3}, 16,17,25$ & $13 \mathrm{a}, 14$ \\
\hline $15-\mathrm{OCH}_{3}$ & 57.2 & $3.13(\mathrm{~s})$ & & 15 & \\
\hline 16 & 134.0 & & & & \\
\hline 17 & 149.9 & $6.65(\mathrm{ddd}, 3.3,1.9,1.4)$ & & $15,16,19,21$ & \\
\hline 18 & 65.1 & $4.68($ dddd, $10.6,5.1,4.7,2.5)$ & 17,19 & & $19 b$ \\
\hline $18-\mathrm{OH}$ & & $5.56(\mathrm{~d}, 5.1)$ & & $17,18,19$ & $19 \mathrm{a}$ \\
\hline $19 \mathrm{a}$ & 39.2 & $1.96(\mathrm{dddd}, 14.1,11.8,10.6,2.5)$ & 18 & 18,20 & $\mathrm{NH}, 18-\mathrm{OH}$ \\
\hline $19 b$ & & $2.26($ dddd, $11.8,4.9,4.7$ 1.9) & 18 & $17,18,20,21$ & 18,20 \\
\hline 20 & 53.2 & $4.55(\mathrm{ddd}, 14.1,9.4,4.9)$ & $\mathrm{NH}, 19$ & 19,21 & $19 b$ \\
\hline 21 & 197.1 & & & & \\
\hline 22 & 12.9 & $1.84(\mathrm{~d}, 1.2)$ & & $1,2,3$ & \\
\hline 23 & 14.2 & $1.62(\mathrm{~s})$ & & $7,8,9$ & \\
\hline 24 & 17.2 & $0.94(\mathrm{~d}, 6.7)$ & 10 & $9,10,11$ & $11-\mathrm{OCH}_{3}$ \\
\hline 25 & 13.9 & $0.69(\mathrm{~d}, 6.7)$ & 14 & $13,14,15$ & \\
\hline
\end{tabular}

HMBC correlations confirmed the assignment of these spin systems and were used to elucidate the linkage points of the molecule. It was established that the oxygenated methines, H-6, H-11, H-12 and H-15, were all next to $O$-methyls (Table 1). The HMBC correlation of the exchangeable proton at $\delta_{\mathrm{H}} 8.02$ to a carbonyl resonance at $\delta_{\mathrm{C}} 171.5$ was consistent with an amide. ${ }^{3} J_{\mathrm{CH}}$ correlations into the same carbonyl were observed from the olefinic proton H-3 and the methyl at $\delta_{\mathrm{H}} 1.84 / \delta_{\mathrm{C}} 12.9(\mathrm{H}-22)$ and ${ }^{2} J_{\mathrm{CH}}$ correlations from both these proton resonances into quaternary carbon resonance at $\delta_{\mathrm{C}} 133.6$ suggested that this carbon was alpha to the amide carbonyl. HMBC correlations from the methyl at $\delta_{\mathrm{H}} 1.62$ to C-7 $\left(\delta_{\mathrm{C}} 78.0\right), \mathrm{C}-9\left(\delta_{\mathrm{C}} 129.4\right)$ and a quaternary carbon resonating at $\delta_{\mathrm{C}} 133.0(\mathrm{C}-8)$ established the connection of the first and second described spin systems through $\mathrm{C}-8$. $\mathrm{H}-11$ showed HMBC correlations to a carbon resonating at 
$\delta_{\mathrm{C}} 82.3(\mathrm{C}-12)$ and $\mathrm{H}-12$ to $\mathrm{C}-11\left(\delta_{\mathrm{C}} 83.8\right)$ that were very hard to discern due to immense overlap in this region, but provided sufficient evidence for a linkage between C-11 to C-12. $\mathrm{H}-15\left(\delta_{\mathrm{H}} 4.15\right)$ showed an additional two unassigned HMBC correlations to the olefinic carbon $\mathrm{C}-17\left(\delta_{\mathrm{H}} 149.9\right)$ as well as a quaternary carbon at $\delta_{\mathrm{H}} 134.0$, linking the spin systems at C-16 with the last spin system. The far downfield double bond resonance of $\mathrm{C}-17$ indicated an electron withdrawing group beta to the double bond proton and a strong HMBC correlation from H-17 to a carbon at $\delta_{\mathrm{C}} 197.1$ determined it to be a carbonyl group. As the downfield methylene proton (H-19b) also showed a correlation to the carbonyl resonance, it was concluded that this fragment consisted of a six-membered 4-hydroxyl-cyclohexenone system. An additional carbon resonance at $\delta_{\mathrm{C}} 156.5$ was clearly visible in the carbon spectrum; however, it showed no HMBC correlations. The chemical shift of this carbon was appropriate for a carbamate and the observation of an additional two heteroatom substituted protons at $\delta_{\mathrm{H}} 6.66$ and $\delta_{\mathrm{H}} 6.30$ supported this assignment. ${ }^{8}$ The downfield chemical shift of the oxygenated proton at $\delta_{\mathrm{H}} 5.16(\mathrm{H}-7)$ suggested that the carbon $\mathrm{C}-7$ was the point of attachment of the carbamate group. With this remaining fragment assigned, the final structure was consistent with the sodium adduct ion $\mathrm{m} / z$ 602.3111 $\left(\mathrm{M}+\mathrm{Na}^{+}\right)$as well as the degrees of hydrogen deficiency calculated from the molecular formula.

An examination of the literature indicated that this compound was an analogue of geldanamycin and had an identical macrolactam ring to the structures of the herbimycin analogues A, D, E and 17,19-dimethylthioherbimycin A. ${ }^{9-11}$ Previously reported geldanamycines and herbimycines contained a 2,6-disubstituted-p-benzoquinone ring or a hydroquinone ring bonded to the macrolactam, whereas the newly described compound, herbimycin G (1), contains a 2,6-disubstituted 4-hydroxyl-cyclohexenone ring and therefore has an additional two stereogenic centres. Consequently, a ROESY spectrum was obtained in order to elucidate the relative configuration of the nine stereogenic centres in 1. ROESY correlations between $\mathrm{H}-6\left(\delta_{\mathrm{H}} 4.57\right)$ and $\mathrm{H}-7\left(\delta_{\mathrm{H}} 5.16\right)$ showed that the two protons were cis to each other, indicating that the O-methyl at C-6 and the carbamate group attached to $\mathrm{C}-7$ were on the $\beta$-face of the molecule. Based on ROESY exchange (antiphase to the ROESY correlations) correlations the exchangeable carbamate $\mathrm{NH}_{2}$ protons could be assigned as $\delta_{\mathrm{H}} 6.30$ and $\delta_{\mathrm{H}} 6.66$. A weak long range correlation between $\mathrm{H}-7$ and the methyl protons $\mathrm{H}-24$ at $\delta_{\mathrm{H}} 0.94$ established the relative configuration of the two spin system relative to C-7. The methyl protons at $\delta_{\mathrm{H}} 0.94$ also correlated to the methoxy protons at $\delta_{\mathrm{H}} 3.35$, and $\mathrm{H}-13 \mathrm{a}\left(\delta_{\mathrm{H}} 1.41\right), \mathrm{H}-14\left(\delta_{\mathrm{H}} 1.51\right)$ and $\mathrm{H}-15$ ( $\left.\delta_{\mathrm{H}} 4.15\right)$ were assigned to the $\alpha$-face of the molecule. A weak ROESY correlation from $\mathrm{H}-15$ to $\mathrm{H}-18\left(\delta_{\mathrm{H}} 4.68\right)$ allowed determination of the configuration of the 4-hydroxyl-cyclohexenone system relative to the macrolactam ring. Further strong correlations between H-18 and H-19b $\left(\delta_{\mathrm{H}} 2.26\right)$, which in turn correlated to $\mathrm{H}-20\left(\delta_{\mathrm{H}} 4.55\right)$, indicated their position to be on the $\alpha$-face. 
The hydroxyl group, H19a $\left(\delta_{\mathrm{H}} 1.96\right)$ and the amide proton $\left(\delta_{\mathrm{H}} 8.02\right)$ faced in the $\beta$-direction. No COSY correlation was observed between $\mathrm{H}-17$ and $\mathrm{H}-18$, indicating that the two protons were at an angle close to $90^{\circ}$ and small long range couplings $(J=1.8 \mathrm{~Hz})$ between $\mathrm{H}-17$ and $\mathrm{H}-19 \mathrm{~b}$ suggested "W" arrangement. ROESY correlations from the methyl resonating at $\delta_{\mathrm{H}} 0.69$ to $\delta_{\mathrm{H}} 1.96$ indicated that the macrolactam folded over the 4-hydroxyl-cyclohexenone. The twodimensional structure was therefore established and redrawn in Maestro 2016 to perform a Monte-Carlo Multiple Minimisation (MCMM) search using the OPLS2005 force field to generate the energy minimised conformation of $\mathbf{1}$ (Figure 1a). The previously observed ROESY correlations were compared to the energy-minimised 3D structure of $\mathbf{1}$, confirming its relative configuration. The absolute configuration of $\mathbf{1}$ was determined by comparison of its experimental and predicted electronic circular dichroism (ECD) spectra, calculated using timedependent density functional theory (TDDFT). A conformational search using MacroModel was performed on the energy minimised structure of $\mathbf{1}$ using the force field parameters as above with extended torsional sampling options. All structures within a $10.0 \mathrm{~kJ} / \mathrm{mol}$ energy window were saved, generating 13 conformers. Geometry optimisation and Gibbs free energy calculations using GAUSSIAN16 at the B3LYP/31G(d) energy level was then performed on each of the conformers. Following this, the ECD spectra of each of the geometry optimised conformers was calculated using GAUSSIAN16 at the same theoretical level. A Boltzmann-weighted ECD spectrum was then calculated using SpecDis 1.7. The experimental ECD spectrum of 1 showed good agreement with the calculated ECD of $(6 S, 7 S, 10 R, 11 R, 12 S, 14 S, 15 R, 18 S, 20 R)-1$ (Figure 1b).
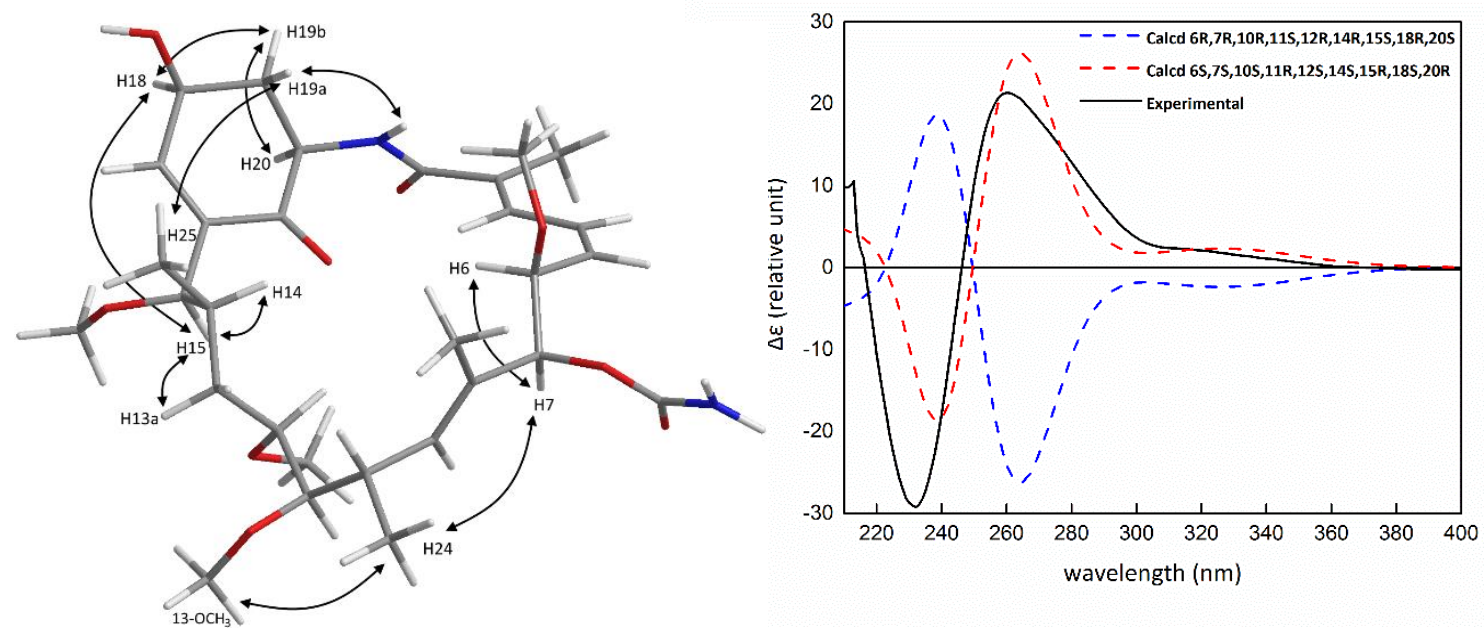

Figure 1. (a) Key ROESY correlations of 1. (b) Comparison of the experimental ECD spectrum of 1 with those calculated at B3LYP/6-31G(d) level 
The configuration of the macrolactam ring is consistent with herbimycin analogues previously reported in the literature, but herbimycin G (1) is the first example of an ansamycin compound that contains a 2,6-disubstituted 4-hydroxyl-cyclohexenone ring. A total of eleven herbimycin analogues, including herbimycin G, have now been reported from Streptomyces sp. strains (Appendix III: Figure IIIH) ${ }^{9-14}$ Benzoquinone ansamycines have been identified as potent inhibitors of the Hsp90, an important cancer target and more recently a novel target for anti-malaria drugs. Geldanamycin had reached clinical trials as an anti-cancer drug, however, the compound had low water solubility and exhibited high liver toxicity. As a result, a number of synthetically modified geldanamycin analogues were made and among those tanespimycin (17-AAG) advanced to phase three of anticancer clinical trials for multiple myeloma. ${ }^{15}$ In herbimycin $\mathrm{G}$ (1) the benzoquinone has been reduced to a 4-hydroxyl-cyclohexenone and thus potentially has better water solubility. Therefore, we assessed the $\operatorname{cog} P$ and other Lipinski parameters of all known herbimycins, as well as a number of related ansamycin compounds (Figure 2, Appendix III: Table IIIA). Compared to geldanamycin, most herbimycin analogues, except for herbimycin D, F and TAN-420E, exhibited similar or lower $\operatorname{clog} P$ values. Herbimycin G (1) and TAN-420B had the lowest $\operatorname{cog} P, 0.68$ and 0.63 , respectively, indicating better water solubility. The molecular weights of all assessed herbimycin and geldanamycin compounds were non-compliant with the Lipinski rule of five that uses four physiochemical properties (molecular weight $<500 \mathrm{Da}$, partition coefficient $\operatorname{cog} P<5$, hydrogen bond donors $<5$ and hydrogen bond acceptors $<10$ ) to assess drug-likeness. ${ }^{16}$ Macrocycles frequently violate the rule of five, but nonetheless have been successful as clinical leads (i.e. rifamycin) or candidates (i.e. erythromycin) and have made their way to the market. ${ }^{17}$ This is due to their high binding affinity, stability and specificity for a molecular target. ${ }^{17}$

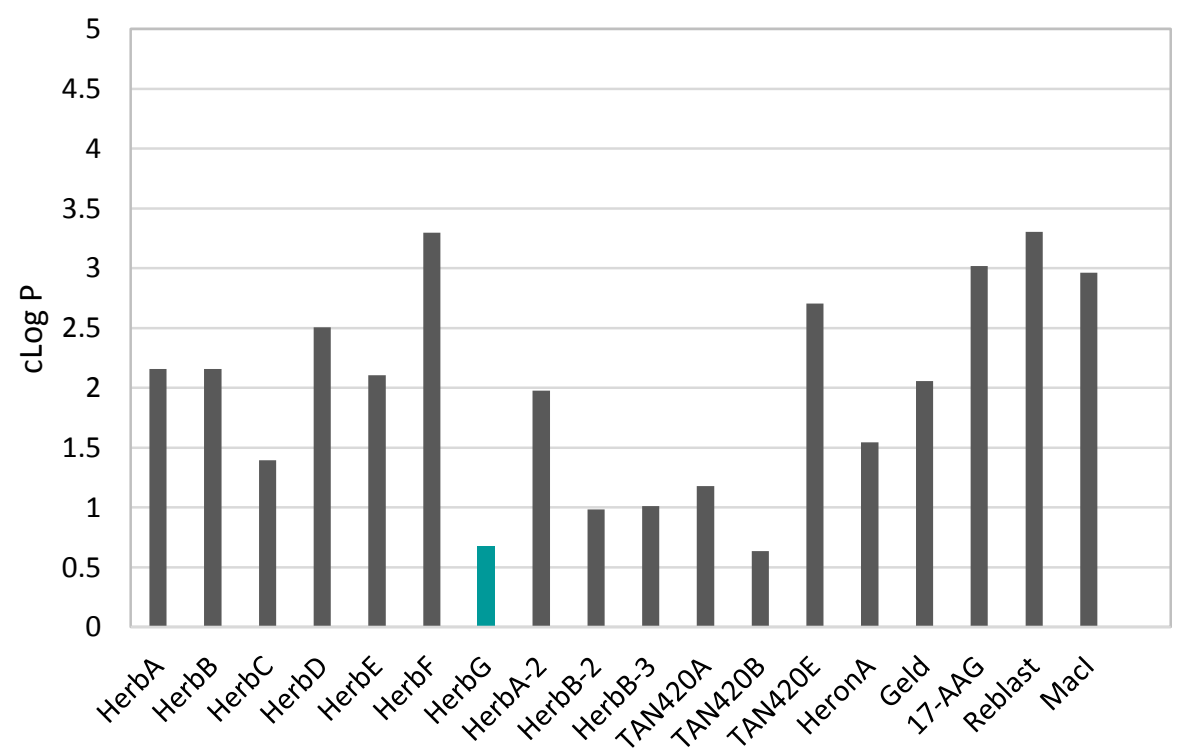

Figure 2. Analysis of clogP values of herbinmcyines and related ansamycin compounds 
In addition to the new herbimycin analogue, GST extracts from the Streptomyces sp. strain USC-16018 yielded the known polyketide, elaiophylin (2), and four diketopiperazines (3 - 6). Obtained spectroscopic data for $\mathbf{3}-\mathbf{6}$, including NMR spectra (Table 2) and specific rotations, were compared to the literature and Cyclo-L-Pro-L-Leu (3), Cyclo-L-Pro-L-Phe (4) Cyclo-L-Pro-L-Val (5) and Cyclo-L-Pro-L-Tyr (6) were identified. ${ }^{18,19}$ Additionally, a positive LR-ESI-MS pseudo-molecular ion at $m / z, 1024.65$ was observed and the molecular formula for 2 was established to be $\mathrm{C}_{54} \mathrm{H}_{88} \mathrm{O}_{18}$. Based on ${ }^{1} \mathrm{H}$, COSY, HSQC and HMBC spectra, a partial structure was elucidated containing 44 protons, 27 carbons and 10 oxygen atoms (Table 3). This only accounted for half the number of carbons and hydrogens, suggesting that $\mathbf{2}$ was a symmetric dimer. Comparison of this NMR data with carbon data documented in the literature identified 2 as the known antibiotic compound elaiophylin. ${ }^{20}$ Several Streptomyces species are known to produce elaiophylin and geldanamycin compounds. Recently, complete genome mining of four geldanamycin producing Streptomyces sp. strains identified up to 56 putative biosynthetic gene clusters and found highly similar geldanamycin and elaiophylin gene clusters in all strains. ${ }^{21}$ Geldanamycin and herbimycin have the same ansamycin carbon skeleton, it is therefore assumed that both biosynthetic pathways exhibit very similar PKS activity. In fact, Rasher and co-workers ${ }^{22}$ proposed that both compounds are "assembled from the 3-amino-5hydroxybenzoic acid (AHBA) starter unit by successive condensation of two-carbon building blocks in seven chain-elongation steps that use one malonyl, four methylmalonyl and two methoxymalonyl extender units" and structural differences are introduced through enzymatic modifications post PKS assembly. ${ }^{22}$ Such findings yet again highlight the biosynthetic potential of Streptomyces species. It is very likely that Streptomyces sp. strain USC-16018 under different laboratory conditions will be able to produce a wide range of additional secondary metabolites.
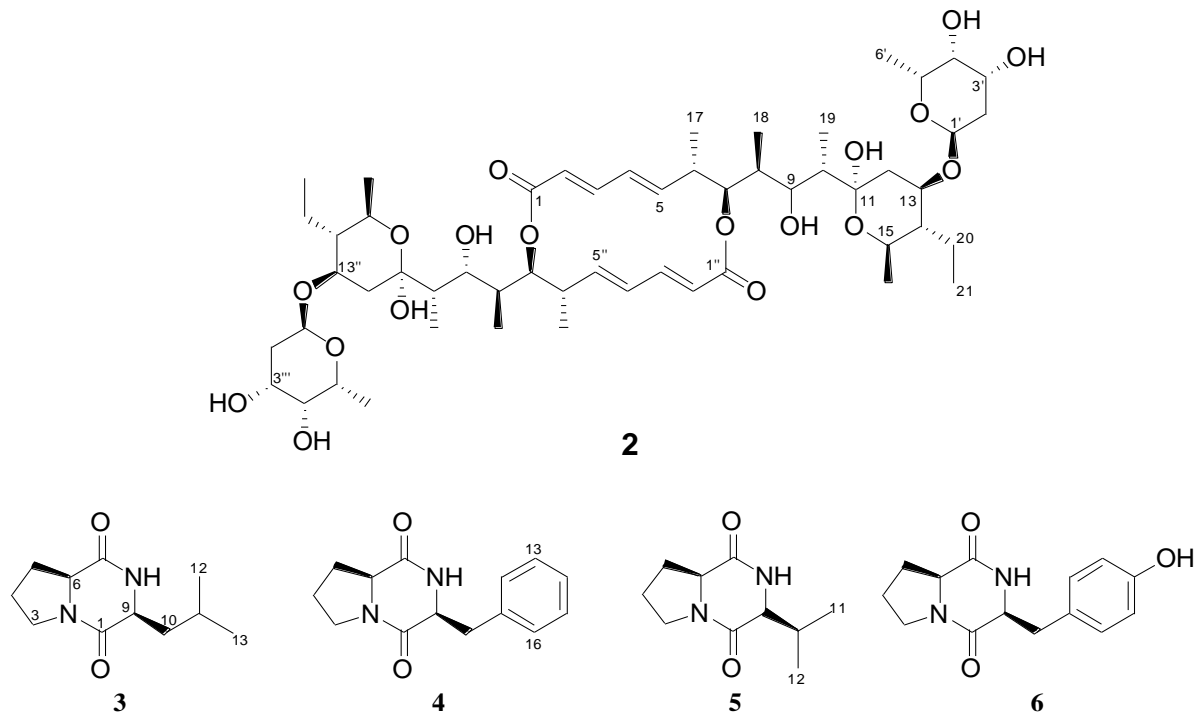
Table 2. NMR data for $3-5\left(500 \mathrm{MHz}\right.$ in DMSO-d $\mathrm{d}_{6}, \delta$ in ppm)

\begin{tabular}{ccccccccc}
\hline \multirow{2}{*}{ position } & $\mathbf{3}$ & \multicolumn{3}{c}{$\mathbf{4}$} & \multicolumn{5}{c}{$\mathbf{5}$} & \multicolumn{3}{c}{$\mathbf{6}$} \\
\cline { 2 - 9 } & $\delta_{\mathrm{H}}$ & $\delta_{\mathrm{C}}$ & $\delta_{\mathrm{H}}$ & $\delta_{\mathrm{C}}$ & $\delta_{\mathrm{H}}$ & $\delta_{\mathrm{C}}$ & $\delta_{\mathrm{H}}$ & $\delta_{\mathrm{C}}$ \\
\hline 1 & & 168.0 & & 165.0 & & 165.9 & & 165.0 \\
3 & $3.38,3.38$ & 44.5 & $3.27,3.38$ & 44.6 & $3.26,3.35$ & 44.4 & $3.36,3.40$ & 44.2 \\
4 & $1.83,1.83$ & 22.0 & 1.73 & 21.0 & $1.79,1.87$ & 22.0 & $1.72,1.84$ & 21.7 \\
5 & $1.90,2.13$ & 27.0 & $1.42,2.01$ & 27.8 & $1.84,2.13$ & 27.6 & $1.40,2.00$ & 27.6 \\
6 & 4.19 & 59.9 & 4.06 & 58.5 & 4.11 & 58.0 & 4.05 & 58.9 \\
7 & & 171.0 & & 169.0 & & 171.1 & & 169.0 \\
8 & 8.01 & & 7.99 & & 7.97 & & 7.86 & \\
9 & 4 & 52.3 & 4.35 & 56.0 & 3.92 & 59.5 & 4.26 & 55.7 \\
10 & $1.37,1.76$ & 37.0 & $3.02,3.06$ & 35.0 & 2.35 & 27.3 & $2.93,2.93$ & 35.6 \\
11 & 1.87 & 23.3 & & 137.0 & 0.85 & 16.4 & & 127.0 \\
12 & 0.86 & 21.2 & 7.27 & 129.0 & 1.01 & 18.2 & 7.05 & 131.0 \\
13 & 0.88 & 22.0 & 7.26 & 127.0 & & & 6.69 & 115.0 \\
14 & & & 7.19 & & & & & 156.0 \\
15 & & & 7.26 & 127.0 & & & 6.69 & 115.0 \\
16 & & & 7.27 & 129.0 & & & 7.05 & 131.0 \\
\hline
\end{tabular}

Table 3. NMR data for $2(800 \mathrm{MHz}$ in DMSO-d $6, \delta$ in ppm)

\begin{tabular}{|c|c|c|}
\hline position & $\delta_{H}$ & $\boldsymbol{\delta}_{\mathrm{C}}$ \\
\hline $1,1^{\prime \prime}$ & & 167.1 \\
\hline $2,2^{\prime \prime}$ & 5.07 & 121.0 \\
\hline $3,3^{\prime \prime}$ & 6.82 & 144.7 \\
\hline $4,4 "$ & 6.12 & 130.4 \\
\hline $5,5^{\prime \prime}$ & 5.65 & 144.8 \\
\hline $6,6^{\prime \prime}$ & 2.48 & 40.7 \\
\hline $7,7 "$ & 5.08 & 75.4 \\
\hline $8,8^{\prime \prime}$ & 1.78 & 36.0 \\
\hline $9,9^{\prime \prime}$ & 3.77 & 69.2 \\
\hline $10,10^{\prime \prime}$ & 1.57 & 42.6 \\
\hline 11,11" & & 99.2 \\
\hline $12,12^{\prime \prime}$ & $0.96,2.25$ & 36.4 \\
\hline 13,13" & 3.78 & 68.8 \\
\hline $14,14 "$ & 1.05 & 48.1 \\
\hline $15,15^{\prime \prime}$ & 3.76 & 65.6 \\
\hline 16,16" & 1.04 & 18.9 \\
\hline $17,17^{\prime \prime}$ & 0.96 & 15.3 \\
\hline $18,18^{\prime \prime}$ & 0.78 & 9.0 \\
\hline 19,19" & 0.86 & 6.8 \\
\hline 20,20" & $1.36,1.60$ & 18.7 \\
\hline 21,21" & 0.78 & 9.0 \\
\hline $1^{\prime}, 1^{\prime \prime \prime}$ & 4.91 & 91.9 \\
\hline $2^{\prime}, 2^{\prime \prime \prime}$ & 1.38 & 32.9 \\
\hline $3^{\prime}, 3^{\prime \prime \prime}$ & 3.37 & 65.0 \\
\hline 4',4"' & 3.38 & 70.0 \\
\hline $5^{\prime}, 5^{\prime \prime \prime}$ & 3.75 & 66.0 \\
\hline $6^{\prime}, 6^{\prime \prime \prime}$ & 1.08 & 17.0 \\
\hline
\end{tabular}


With the aim to discover marine actinomycete-derived anti-plasmodial compounds, compounds $\mathbf{1}$ - 5 were screened for activity against chloroquine-sensitive (3D7) and chloroquine-resistant (Dd2) strains of Plasmodium (Table 4). Elaiophylin (2) inhibited both the 3D7 and Dd2 parasites with similar potency, $\mathrm{IC}_{50}=778 \mathrm{nM}$ and $598 \mathrm{nM}$, respectively. Antiplasmodial activity $\left(\mathrm{IC}_{50} 214 \mathrm{nM}\right)$ against the 3D7 strain was previously reported in the literature, which was 3.6 fold lower than here presented. ${ }^{23}$ Herbimycin G showed no cytotoxicity and significant parasite inhibition of above $77 \%$ for 3D7 at $40 \mu \mathrm{M}$. Against the chloroquine-resistant strain Dd2, 1 showed $82 \%$ inhibition, which was similar to the observed activity for $\mathbf{2}$, however, the curve did not plateau at the highest tested dose and therefore no $\mathrm{IC}_{50}$ could be determined (Figure 3). The remaining compounds were devoid of antimalarial or cytotoxic activity, while the activity of the reference compounds was in the expected range (Table 4).

Table 4. Anti-plasmodial activity and cytotoxicity of compounds 1 - 6

\begin{tabular}{cccc}
\hline \multirow{2}{*}{ Compound } & \multicolumn{3}{c}{ \% inhibition at $\mathbf{4 0} \boldsymbol{\mu M}\left(\mathbf{I C}_{\mathbf{5 0}}\right.$ in $\left.\mathbf{~ n M}\right)$} \\
\cline { 2 - 4 } & $3 \mathrm{D} 7$ & Dd2 & HEK-293 \\
\hline Herbimycin G (1) & 77.2 & 81.7 & IA \\
Elaiophylin (2) & $96.6(777.9)$ & $86.1(598.5)$ & $101.9(1445)$ \\
Cyclo-Pro-Leu (3) & 45.9 & 39.0 & IA \\
Cyclo-Pro-Phe (4) & IA & IA & IA \\
Cyclo-Pro-Val (5) & IA & IA & IA \\
Cyclo-Pro-Tyr (5) & IA & IA & IA \\
Artesunate & $99.6(0.9)$ & $98.4(1.3)$ & 50.6 \\
Chloroquine & $98.8(10.0)$ & $96.5(87.9)$ & 40.9 \\
DHA & $99.9(0.4)$ & $98.0(0.6)$ & 37.9 \\
Puromycin & $99.0(148.9)$ & $99.1(114.4)$ & $102.6(1409.5)$ \\
Pyrimethamine & $98.7(4.7)$ & 23.2 & 68.4 \\
Pyronaridine & $99.9(7.4)$ & $97.8(8.3)$ & $98.7(2825.5)$ \\
\hline
\end{tabular}

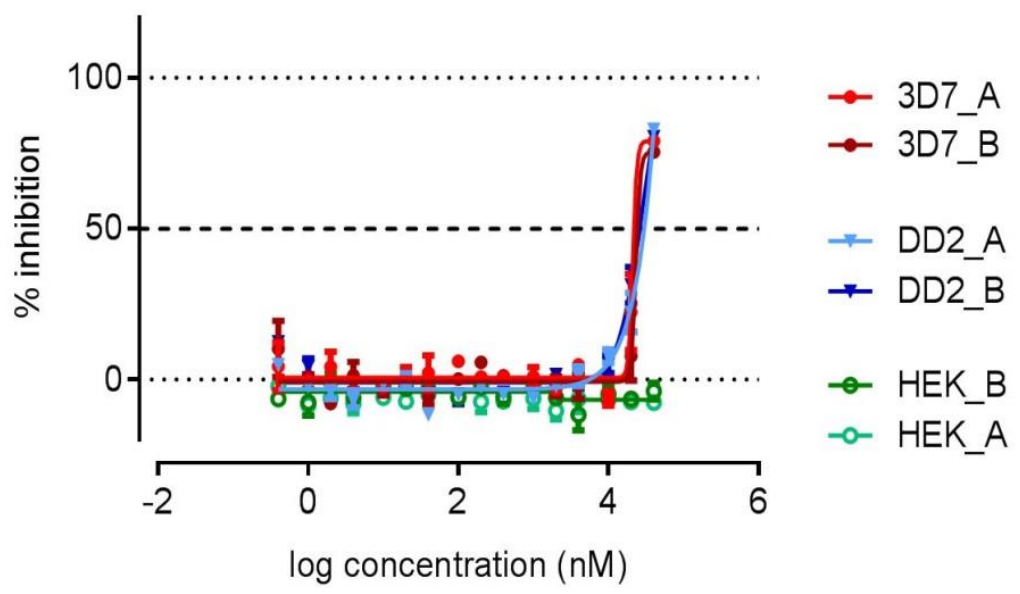

Figure 3. Dose-response curve for 1 against 3D7, Dd2 and HEK-293 in duplicates 
These results show that marine actinomycetes are still a valuable source for the discovery of new secondary metabolites. Several herbimycin, geldanamycin and other ansamycin compounds have been reported previously from terrestrial and marine Streptomyces species. To our knowledge, the new compound, herbimycin G (1), is the first example of such a compound containing a 2,6-disubstituted 4-hydroxyl-cyclohexenone ring. Herbimycin G exhibited weak anti-plasmodial activity with no cytotoxicity. We investigated the physiochemical properties of reported herbimycins and related analogues and found that $\mathbf{1}$ had a lower $\operatorname{cog} P$ compared to the other herbimycin analogues, potentially related to the different ring structure, suggesting good bioavailability. As herbimycin G did not show cytotoxicity against HEK293, it appears promising to test this compound for other biological activity, such as anti-tumour, in the future.

\subsection{Experimental section}

\subsubsection{General Experimental Section}

Extracts were dried with a rotary evaporator (Christ Rotational Vacuum Concentrator RVC 2-25 CD plus). NMR spectra were recorded at $25{ }^{\circ} \mathrm{C}$ on a Bruker BioSpin GmbH 800 $\mathrm{MHz}$ spectrometer equipped with a cryoprobe. The ${ }^{1} \mathrm{H}$ and ${ }^{13} \mathrm{C}$ chemical shifts were referenced to the DMSO- $d_{6}$ solvent peak at $\delta_{\mathrm{H}} 2.50$ and $\delta_{\mathrm{C}} 39.5$, respectively. Standard parameters were used for obtained 2D NMR spectra, including gCOSY, gHSQC, gHMBC and ROESY. HRESIQTOFMS were recorded on a 6530 Accurate Mass Q-TOF (Agilent Technologies) workstation using positive electrospray ionisation, with mobile phase $\mathrm{ACN} / \mathrm{H}_{2} \mathrm{O}$ containing $0.1 \%$ formic acid. A LaChrom Merck Hitachi L7100 series pump equipped with a Hitachi L7450 PDA detector was used for HPLC separations. Prior to HPLC separation, the microbial crude extract was adsorbed onto Alltech Davisil 35- $75 \mu \mathrm{m} 150 \AA \mathrm{C}_{18}$. All separations were performed with a Thermo Hypersil-Keystone Betasil $5 \mu \mathrm{m} \AA \mathrm{C}_{18}$ HPLC column $(21.2 \mathrm{~mm} \times 150 \mathrm{~mm})$. Optical rotations were measured on a JASCO P-1020 polarimeter. UV spectra were obtained on a Shimadzu UV-1800 UV/vis spectrophotometer. CD spectra were recorded on a JASCO J-715 spectropolarimeter. EtOAc used for extractions was Honeywell HPLC grade. All solvents used for HPLC, UV, and $[\alpha]_{D}$-measurements were Scharlau HPLC grade. For MS, Fisher Scientific LC/MS grade acetonitrile was used and $\mathrm{H}_{2} \mathrm{O}$ was Millipore Milli-Q PF filtered.

\subsubsection{Biological material}

Streptomyces strain USC-16018 was isolated from the ascidian Symplegma rubra collected on July 15, 2015, Hastings Point, NSW, Australia. The ascidian specimen was identified by Merrick Ekins (Queensland Museum). The Streptomyces strain was isolated using selective 
media and identified via 16S rRNA sequencing (as described in Chapter 2). The 16S sequence was deposited in GenBank (accession number MF773774).

\subsubsection{Fermentation, Extraction and Isolation}

Strain USC-16018 was fermented on 55 OMA (20 g oatmeal, $20 \mathrm{~g}$ bacteriological agar, $3 \mathrm{~g}$ yeast extract, $1 \mathrm{~L}$ deionised $\mathrm{H}_{2} \mathrm{O}$ ) and 55 GST ( $5 \mathrm{~g}$ glucose, $2 \mathrm{~g}$ starch, $3 \mathrm{~g}$ beef extract, $5 \mathrm{~g}$ yeast extract, $5 \mathrm{~g}$ tryptone, $4 \mathrm{~g} \mathrm{CaCO}_{3}, 4 \mathrm{~g} \mathrm{NaCl}, 1 \mathrm{~g} \mathrm{NaSO}_{4}, 0.5 \mathrm{~g} \mathrm{KCl}, 2 \mathrm{~g} \mathrm{MgCl}, 0.5 \mathrm{~g}$ $\mathrm{KH}_{2} \mathrm{PO}_{4}, 20 \mathrm{~g}$ bacteriological agar) agar plates for 2 weeks at $28{ }^{\circ} \mathrm{C}$. Culture plates were exhaustively extracted by shaking in ethyl acetate. The extracts were dried under reduced vacuum to yield $1.6 \mathrm{~g}$ of dark brown gum for OMA cultures and $0.8 \mathrm{~g}$ of light red gum for GST cultures. Each of the extracts was dissolved in a small amount of $\mathrm{MeOH}$ and $\mathrm{C}_{18}$ silica gel was added in a 1:2 ratio. The solvent was evaporated and the extract adsorbed on the $\mathrm{C}_{18}$ was transferred to a refillable HPLC guard column $(10 \mathrm{~mm}$ x $20 \mathrm{~mm})$ which was attached to a $\mathrm{C}_{18}$ Betasil HPLC column. At a flow rate of $9 \mathrm{~mL} / \mathrm{min}$, isocratic HPLC conditions of $10 \%$ aqueous $\mathrm{MeOH}$ were initially employed for $10 \mathrm{~min}$, followed by a linear gradient over $40 \mathrm{~min}$ from $10 \%$ to $100 \% \mathrm{MeOH}$, finishing with another $10 \mathrm{~min}$ isocratic conditions at $100 \% \mathrm{MeOH}$. Sixty 1-minute-fractions were collected and analysed by ${ }^{1} \mathrm{H}$ NMR spectroscopy. Of the OMA extract, fractions 31- 35 were combined and further purified by eluting with a gradient from 25 to $60 \%$ aqueous $\mathrm{MeOH}$ over $40 \mathrm{~min}$ to yield $0.9 \mathrm{mg}$ of compound $\mathbf{1}$. Fraction 46 and 26 of the GST extract HPLC separation yielded $0.6 \mathrm{mg}$ and $0.7 \mathrm{mg}$ of compound $\mathbf{2}$ and $\mathbf{3}$ respectively. Fractions $20-25$ were combined and repurified to yield $1.2 \mathrm{mg}$ of compound 4 and $2.1 \mathrm{mg}$ of compound 5. $0.9 \mathrm{mg}$ of compound $\mathbf{2}$ were also detected in the OMA extract.

Herbimycin $\mathrm{G}(\mathbf{1})$ : $\mathrm{C}_{30} \mathrm{H}_{46} \mathrm{~N}_{2} \mathrm{O}_{9}$; brown solid; $[\alpha]_{\mathrm{D}}+8.6$ (c $\left.0.007 \mathrm{~g} / 100 \mathrm{~mL}, \mathrm{MeOH}\right), \mathrm{UV}$ $(\mathrm{MeOH}) \lambda_{\max }(\log \varepsilon) 245$ (1.93) nm; ${ }^{1} \mathrm{H}$ NMR (800 MHz, DMSO) and ${ }^{13} \mathrm{C}$ NMR (200 MHz, DMSO) data, Table 1; (+)-HR-ESIMS $m / z 601.3111[\mathrm{M}+\mathrm{Na}]^{+}$(calculated for $\mathrm{C}_{30} \mathrm{H}_{46} \mathrm{~N}_{2} \mathrm{NaO}_{9}$, 601.3096); all 1D and 2D NMR spectra in Appendix III.

Elaiophylin (2): $\mathrm{C}_{54} \mathrm{H}_{88} \mathrm{O}_{18} ;{ }^{1} \mathrm{H}$ NMR (800 MHz, DMSO) and ${ }^{13} \mathrm{C}$ NMR (200 MHz, DMSO) data, Table 3; (+)-LR-ESIMS $m / z$ 1024.6 $[\mathrm{M}+\mathrm{H}]^{+}$(calculated for $\mathrm{C}_{54} \mathrm{H}_{88} \mathrm{O}_{18}, 1024.60$ ).

Cyclo-L-Pro-L-Leu (3): $\mathrm{C}_{11} \mathrm{H}_{18} \mathrm{~N}_{2} \mathrm{O}_{2}$; yellow solid; $[\alpha]_{\mathrm{D}}-3.1(c 0.02 \mathrm{~g} / 100 \mathrm{~mL}, \mathrm{MeOH})$; UV $(\mathrm{MeOH}) \lambda_{\max }(\log \varepsilon) 277(0.71) \mathrm{nm}, 329(-0.01) \mathrm{nm} ;{ }^{1} \mathrm{H}$ NMR (800 MHz, DMSO) and ${ }^{13} \mathrm{C}$ NMR (200 MHz, DMSO) data, Table 2.

Cyclo-L-Pro-L-Phe (4): $\mathrm{C}_{14} \mathrm{H}_{16} \mathrm{~N}_{2} \mathrm{O}_{2}$; yellow solid $[\alpha]_{\mathrm{D}}-1.76$ (c $\left.0.008 \mathrm{~g} / 100 \mathrm{~mL}, \mathrm{MeOH}\right)$, $\mathrm{UV}\left(\mathrm{MeOH} \lambda_{\max }(\log \varepsilon) 216\right.$ (1.58) nm; ${ }^{1} \mathrm{H}$ NMR (800 MHz, DMSO) and ${ }^{13} \mathrm{C}$ NMR (200 MHz, DMSO) data, Table 2. 
Cyclo-L-Pro-L-Val (5): $\mathrm{C}_{10} \mathrm{H}_{14} \mathrm{~N}_{2} \mathrm{O}_{2}$; yellow solid; $[\alpha]_{\mathrm{D}}-1.3$ (c $\left.0.01 \mathrm{~g} / 100 \mathrm{~mL}, \mathrm{MeOH}\right) ; \mathrm{UV}$ $\left(\mathrm{MeOH} \lambda_{\max }(\log \varepsilon) 215(1.72) \mathrm{nm} ;{ }^{1} \mathrm{H}\right.$ NMR $(800 \mathrm{MHz}, \mathrm{DMSO})$ and ${ }^{13} \mathrm{C}$ NMR (200 MHz, DMSO) data, Table 2.

Cyclo-L-Pro-L-Tyr (6): $\mathrm{C}_{14} \mathrm{H}_{16} \mathrm{~N}_{2} \mathrm{O}_{3}$; yellow solid; $[\alpha]_{\mathrm{D}}-1.3$ (c $\left.0.01 \mathrm{~g} / 100 \mathrm{~mL}, \mathrm{MeOH}\right) ; \mathrm{UV}$ $\left(\mathrm{MeOH} \lambda_{\max }(\log \varepsilon) 215(1.72) \mathrm{nm} ;{ }^{1} \mathrm{H}\right.$ NMR $(800 \mathrm{MHz}, \mathrm{DMSO})$ and ${ }^{13} \mathrm{C}$ NMR (200 MHz, DMSO) data, Table 2.

\subsubsection{Molecular modelling calculations}

The generation of conformers was performed using Schrödinger MacroModel 2016 using the protocol reported by Willoughby and co-workers. ${ }^{24}$ First-principles calculations based on density functional theory (DFT) were carried out to optimize the atomic structures at the B3LYP/6-31G(d) level with the GAUSSIAN 16 suite of programs. ${ }^{25}$ Electronic transition and rational strength were calculated using the time-dependent DFT (TDDFT) method at the same theoretical level. The solvent effect in $\mathrm{MeOH}$ solution was considered during all calculations using the Polarizable Continuum Model (PCM). ${ }^{26}$ Boltzmann-weighted UV and ECD were calculated using the freely available software SpecDis ${ }^{27}$ and GaussSum ${ }^{28}$ using a sigma/gamma value of $0.25 \mathrm{eV}$. Lipinski parameters were calculated in ChemBio3D Ultra 14.0.

\subsubsection{Biological activity testing}

P. falciparum parasites (3D7 and Dd2 strains) were grown in RPMI 1640 supplemented with $25 \mathrm{mM}$ HEPES, 5\% AB human male serum, $2.5 \mathrm{mg} / \mathrm{ml}$ Albumax II, and $0.37 \mathrm{mM}$ hypoxanthine. Parasites were subjected to two rounds of sorbitol synchronization before undergoing compound treatment. Ring stage parasites were exposed to the experimental compounds in 384-wells imaging microplates (PerkinElmer CellCarrier), as previously described. ${ }^{29}$ Plates were incubated for $72 \mathrm{~h}$ at $37{ }^{\circ} \mathrm{C}, 90 \% \mathrm{~N}_{2}, 5 \% \mathrm{CO}_{2}, 5 \% \mathrm{O}_{2}$, then the parasites were stained with 2-(4-amidinophenyl)-1H -indole-6-carboxamidine (DAPI), and imaged using an Opera QEHS micro-plate confocal imaging system (PerkinElmer). Images were analysed as previously described. ${ }^{29}$

Human Embryonic Kidney cells (HEK293) were maintained in DMEM medium supplemented with 10\% FBS. HEK293 cells were exposed to the compounds in TC-treated 384wells plates (Falcon) as previously described..$^{30}$ Plates were incubated for $72 \mathrm{~h}$ at $37{ }^{\circ} \mathrm{C}, 5 \% \mathrm{CO}_{2}$, then the media was removed from the wells and replaced with an equal volume of $44 \mu \mathrm{M}$ resazurin. After an additional 5-6 hours incubation at standard conditions, the total fluorescence (excitation/emission: $530 \mathrm{~nm} / 595 \mathrm{~nm}$ ) was measured using the Envision plate reader (PerkinElmer). 
Raw data was normalized using the in-plate positive and negative controls to obtain normalized \% inhibition data, which was then used to calculate $\mathrm{IC}_{50}$ values, through a 4 parameter logistic curve fitting in GraphPad Prism. The experiments were carried out in two independent biological replicates, each consisting of two technical replicates.

\subsection{References}

(1) World Health Organization, World malaria report 2016; Available online: http://www.who.int/malaria/publications/world-malaria-report-2016/report/en/

(2) Tuteja, R. FEBS J. 2007, 274, 4670.

(3) Shahinas, D.; Folefoc, A.; Pillai, D. R. Pathogens 2013, 2, 33.

(4) Michalakis, Y.; Renaud, F. Nature 2009, 462, 298.

(5) Banumathy, G.; Singh, V.; Pavithra, S. R.; Tatu, U. J. Biol. Chem. 2003, 278, 18336.

(6) Kumar, R.; Musiyenko, A.; Barik, S. Malar. J. 2003, 2, 30.

(7) Mout, R.; Xu, Z.-D.; Wolf, A. K.; Davisson, V. J.; Jarori, G. K. Malar. J. 2012, 11, 54.

(8) Pretsch, E.; Bühlmann, P.; Affolter, C.; Pretsch, E.; Bhuhlmann, P.; Affolter, C. Structure determination of organic compounds; Springer, 2009; Vol. 13.

(9) Omura, S.; Iwai, Y.; Takahashi, Y.; Sadakane, N.; Nakagawa, A.; Oiwa, H.; Hasegawa, Y.; Ikai, T. J. Antibiot. 1979, 32, 255.

(10) Shaaban, K. A.; Wang, X.; Elshahawi, S. I.; Ponomareva, L. V.; Sunkara, M.; Copley, G. C.; Hower, J. C.; Morris, A. J.; Kharel, M. K.; Thorson, J. S. J. Nat. Prod. 2013, 76, 1619.

(11) Li, S.; Cui, J.; Lu, X.; Zheng, Z.; Liu, X.; Ni, S.; Wang, Y.; Wu, L. J. Antibiot. 2013, 66, 499.

(12) Iwai, Y.; Nakagawa, A.; Sadakane, N.; Omura, S.; Oiwa, H.; Matsumoto, S.; Takahashi, M.; Kiai, T.; Ochiai, Y. J. Antibiot. 1980, 33, 1114.

(13) Zhao, W.; Jiang, B.; Wu, L.; Nan, Y.; Cui, J.; Yu, L.; Wei, Y.; Li, J.; Shan, G. J. Antibiot. 2015, 68, 476.

(14) Raju, R.; Piggott, A. M.; Khalil, Z.; Bernhardt, P. V.; Capon, R. J. Tetrahedron Lett. 2012, 53, 1063.

(15) Dimopoulos, M.-A.; Mitsiades, C. S.; Anderson, K. C.; Richardson, P. G. Clin. Lymphoma, Myeloma Leuk. 2011, 11, 17.

(16) Lipinski, C. A.; Lombardo, F.; Dominy, B. W.; Feeney, P. J. Adv. Drug Delivery Rev. 1997, 23, 3.

(17) Levin, J. I. Macrocycles in Drug Discovery; Royal Society of Chemistry, 2014; Vol. 40.

(18) Jayatilake, G. S.; Thornton, M. P.; Leonard, A. C.; Grimwade, J. E.; Baker, B. J. J. Nat. Prod. 1996, 59, 293

(19) Puopolo, G.; Cimmino, A.; Palmieri, M.; Giovannini, O.; Evidente, A.; Pertot, I. J. Appl. Microbiol. 2014, 117, 1168.

(20) Hammann, P.; Kretzschmar, G. Magn. Reson. Chem. 1991, 29, 667.

(21) Yin, M.; Jiang, M.; Ren, Z.; Dong, Y.; Lu, T. J. Biotechnol. 2017, 252, 27. 
(22) Rascher, A.; Hu, Z.; Buchanan, G. O.; Reid, R.; Hutchinson, C. R. Applied and Environ. Microbiol. 2005, 71, 4862.

(23) Supong, K.; Sripreechasak, P.; Tanasupawat, S.; Danwisetkanjana, K.; Rachtawee, P.; Pittayakhajonwut, P. Appl. Microbiol. Biotechnol. 2017, 101, 533.

(24) Willoughby, P. H.; Jansma, M. J.; Hoye, T. R. Nat. Protoc. 2014, 9, 643.

(25) Gaussian 16, Frisch, M. J.; Trucks, G. W.; Schlegel, H. B.; Scuseria, G. E.; Robb, M. A.; Cheeseman, J. R.; Scalmani, G.; Barone, V.; Petersson, G. A.; Nakatsuji, H.; Li, X.; Caricato, M.; Marenich, A. V.; Bloino, J.; Janesko, B. G.; Gomperts, R.; Mennucci, B.; Hratchian, H. P.; Ortiz, J. V.; Izmaylov, A. F.; Sonnenberg, J. L.; Williams; Ding, F.; Lipparini, F.; Egidi, F.; Goings, J.; Peng, B.; Petrone, A.; Henderson, T.; Ranasinghe, D.; Zakrzewski, V. G.; Gao, J.; Rega, N.; Zheng, G.; Liang, W.; Hada, M.; Ehara, M.; Toyota, K.; Fukuda, R.; Hasegawa, J.; Ishida, M.; Nakajima, T.; Honda, Y.; Kitao, O.; Nakai, H.; Vreven, T.; Throssell, K.; Montgomery Jr., J. A.; Peralta, J. E.; Ogliaro, F.; Bearpark, M. J.; Heyd, J. J.; Brothers, E. N.; Kudin, K. N.; Staroverov, V. N.; Keith, T. A.; Kobayashi, R.; Normand, J.; Raghavachari, K.; Rendell, A. P.; Burant, J. C.; Iyengar, S. S.; Tomasi, J.; Cossi, M.; Millam, J. M.; Klene, M.; Adamo, C.; Cammi, R.; Ochterski, J. W.; Martin, R. L.; Morokuma, K.; Farkas, O.; Foresman, J. B.; Fox, D. J. Wallingford, CT, 2016.

(26) Tomasi, J.; Mennucci, B.; Cammi, R. Chem. Rev. 2005, 105, 2999.

(27) Bruhn, T.; Schaumloeffel, A.; Hemberger, Y.; Bringmann, G. Chirality 2013, 25, 243.

(28) O'boyle, N. M.; Tenderholt, A. L.; Langner, K. M. J. Comput. Chem. 2008, 29, 839.

(29) Duffy, S.; Avery, V. M. Am. J. Trop. Med. Hyg. 2012, 86, 84.

(30) Fletcher, S.; Avery, V. M. Malar. J. 2014, 13, 343. 


\section{CHAPTER 6 - Naseseazine C, a new anti-plasmodial dimeric diketopiperazine from a marine sediment derived \\ Streptomyces sp.}

\section{STATEMENT OF CONTRIBUTION TO CO-AUTHORED PUBLISHED PAPER}

This chapter has been published as a co-authored paper in the journal Tetrahedron Letters. The bibliographic details of the co-authored paper, including all authors, are:

Buedenbender, L., Grkovic, T., Duffy, S., Kurtböke, D.I., Avery, V.M., Carroll, A.R., Naseseazine $\mathrm{C}$, a new anti-plasmodial dimeric diketopiperazine from a marine sediment derived Streptomyces sp., Tetrahedron Letters, 2016, 57, 5893-5895. doi: http://dx.doi.org/10.1016/j.tetlet.2016.11.071

The author this thesis, L. Buedenbender, performed microbial fermentations, extraction, isolation and structure elucidation of the natural products; and prepared the manuscript. The coauthors T. Grkovic, D. I. Kurtböke and A. R. Carroll are the supervisors to the $\mathrm{PhD}$ project and provided advice on structure elucidation and feedback to the manuscript. S. Duffy and V. M. Avery performed anti-plasmodial activity testing of the isolated compounds.

(Signed

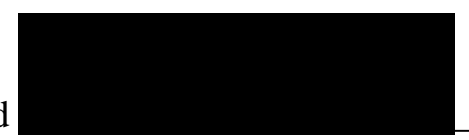

(Date) $\underline{01 / 09 / 2017}$

Larissa Buedenbender

(Countersigned)

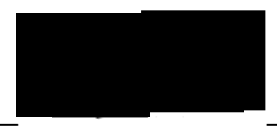

(Date) $\underline{31 / 08 / 2017}$

Corresponding author of paper: Anthony R. Carroll

(Countersigned)

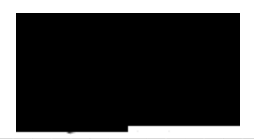

(Date) $\underline{31 / 08 / 2017}$

Principle supervisor: Anthony R. Carroll 


\subsection{Abstract}

A new dimeric diketopiperazine, naseseazine $\mathrm{C}$, and the known naseseazines $\mathrm{A}$ and $\mathrm{B}$ were isolated from solid culture extracts of an Australian marine sediment derived Streptomyces species. Naseseazine $\mathrm{C}$ displayed a new C-6'/C-3 linkage between the two diketopiperazine subunits and showed moderate activity against the chloroquine-sensitive malaria parasite, Plasmodium falciparum (3D7 strain).

\subsection{Introduction}

Naseseazines A and B were originally isolated from the Fijian marine sediment derived actinomycete Streptomyces (CMB-MQ030) and reported to possess diketopiperazine skeletons derived from tryptophan and either proline or alanine. ${ }^{1}$ The unique feature of the structures is their dimeric nature, with the two diketopiperazine units joined by a bond between the dihydroindole C-3 carbon of one monomer and the aryl C-7' carbon of the second monomer. Since their initial discovery, the two naseseazines have been the target of total synthesis and in 2011 Kim and Movassaghi successfully synthesised the proposed structures. ${ }^{2}$ However, the spectroscopic properties of these compounds differed from those of the natural products and based upon this observation diastereomers at $\mathrm{C}-11, \mathrm{C}-15, \mathrm{C}-11^{\prime}$ and $\mathrm{C}-15^{\prime}$ were also made. These compounds possessed identical spectroscopic properties to naseseazines A and B and therefore allowed the structures (including absolute configurations) of the natural products to be corrected (1-2, Figure 1). Additionally, a regioisomer named iso-naseseazine B (3, Figure 1) was also synthesised. This compound differed from naseseazine B through a regiochemical shift of the bond linking the two monomers to C-6'/C-3 (rather than C-7'/C-3). Very recently another molecule also named iso-naseseazine B (4, Figure 1) from a Yellow Sea sediment-derived Streptomyces species was reported. ${ }^{3}$ This compound has been proposed to differ from naseseazine B by the inversion of chirality at the two stereogenic centres C-2 and C-3. In the course of our studies to identify new actinomycete derived compounds from Australian marine environments, we isolated a molecule with the same spectroscopic properties as that reported for 4. However, interpretation of $2 \mathrm{D}$ NMR spectroscopic data led us to the conclusion that the proposed structure reported for $\mathbf{4}$ was incorrect. This manuscript describes the isolation of $\mathbf{5}$ and structural revision of compound $\mathbf{4}$; we have named the new compound naseseazine $\mathrm{C}(\mathbf{5})$. 

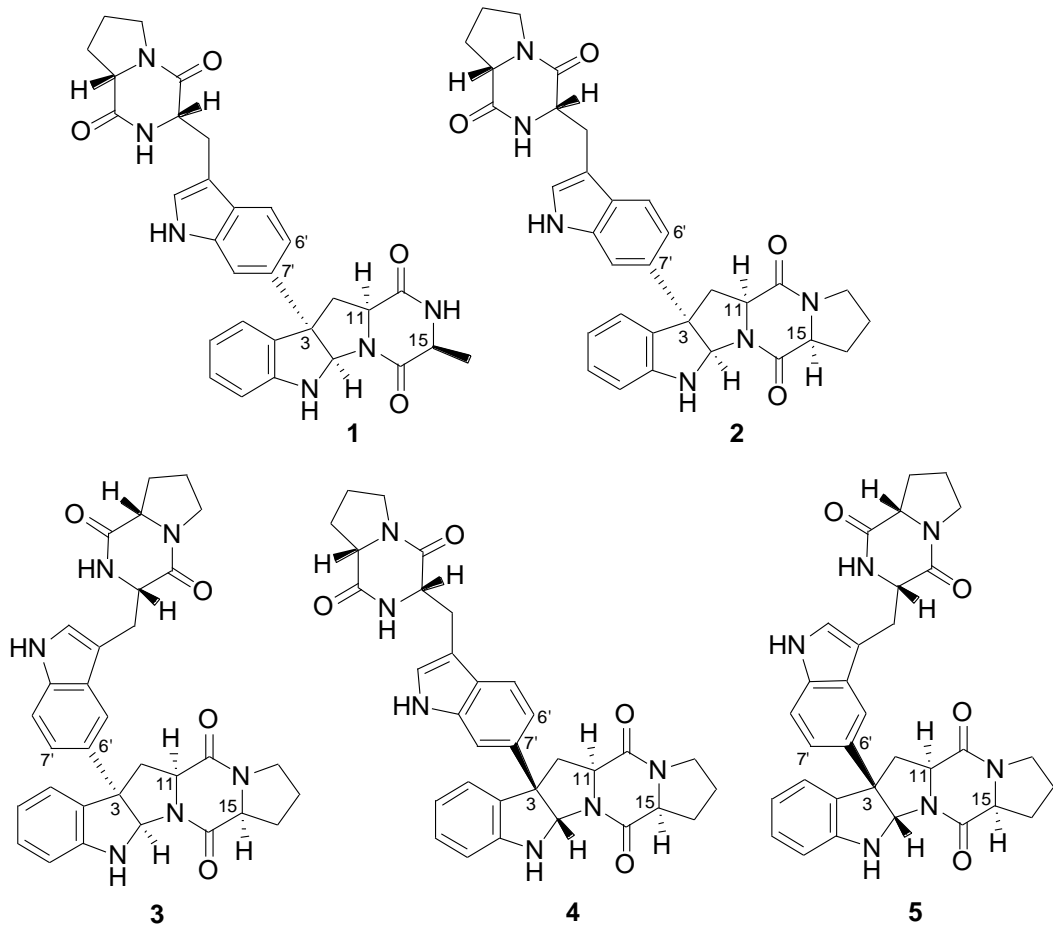

Figure 1. Naseseazine compounds

\subsection{Results and discussion}

The Streptomyces sp. (USC-636) (GenBank Accession Number KX379154) was isolated from marine sediment obtained on the Sunshine Coast, QLD, Australia. 16S rRNA sequencing revealed the close relationship of the isolate to the previously isolated Streptomyces sp. (AMS662) from sediments collected in the western Gulf of California. Streptomyces isolate (USC-636) was cultivated on CYC agar (DSMZ 550, www.dsmz.de). For further chemical analysis, fifty two agar plates were incubated for two weeks at $28{ }^{\circ} \mathrm{C}$ and then extracted with ethyl acetate. The ${ }^{1} \mathrm{H}$ NMR spectrum of the extract revealed unusual aromatic and alkaloid moieties and on the basis of these observations the sample was selected for further investigation. The ethyl acetate extract was purified by HPLC using $\mathrm{H}_{2} \mathrm{O} / \mathrm{MeOH}$ to yield $0.05 \mathrm{mg}$ of naseseazine C (5), as well as $0.1 \mathrm{mg}$ and $0.08 \mathrm{mg}$ of the known compounds naseseazines A (1) and B (2) respectively.

Naseseazine C (5) was isolated as an optically active yellow oil $[\alpha]_{\mathrm{D}}-18.5 .^{4} \mathrm{~A}$ pseudomolecular ion at $\mathrm{m} / \mathrm{z} 565.2576$ obtained from HRESI-QTOFMS indicated 5 had the same molecular formula $\mathrm{C}_{32} \mathrm{H}_{32} \mathrm{~N}_{6} \mathrm{O}_{4}$ as naseseazine $\mathrm{B}$. The NMR data, however, showed distinct differences, particularly in the resonances of the aromatic region (Table 1). 
Table 1. NMR data for naseseazine C (5) (800 MHz in DMSO- $d_{6}, \delta$ in ppm

\begin{tabular}{|c|c|c|c|c|}
\hline Position & $\delta_{\mathrm{H}}$, multiplet, $(J$ in $\mathbf{H z})$ & $\boldsymbol{\delta}_{\mathrm{C}}$ & HMBC & ROESY \\
\hline 1 & $6.56, \mathrm{~s}$ & & 3,4 & 2 \\
\hline 2 & $5.62, \mathrm{~s}$ & 81.1 & $3,4,9$ & $1,12 \mathrm{a}, 5^{\prime}, 7^{\prime}$ \\
\hline 3 & & 59.7 & & \\
\hline 4 & & 134.1 & & \\
\hline 5 & $7.16, \mathrm{dd},(7.4,1.2)$ & 125.8 & 9 & $5^{\prime}, 12 b$ \\
\hline 6 & $6.61, \mathrm{td},(7.4,1.0)$ & 118.1 & 4,8 & \\
\hline 7 & $6.98, \mathrm{td},(7.7,1.2)$ & 128.1 & 5,9 & \\
\hline 8 & $6.65, \mathrm{dd},(7.8,1.5)$ & 111.4 & 4,6 & \\
\hline 9 & & 149.3 & & \\
\hline \multicolumn{5}{|l|}{10} \\
\hline 11 & $4.16, \mathrm{~m}$ & 60.1 & & $12 \mathrm{a} / \mathrm{b}$ \\
\hline $12 \mathrm{a}$ & $2.69, \mathrm{~m}$ & 40.0 & $3,4,13$ & $2,11,5^{\prime}, 7^{\prime}$ \\
\hline $12 \mathrm{~b}$ & $3.04, \mathrm{~m}$ & & 2 & 11 \\
\hline 13 & & 165.3 & & \\
\hline \multicolumn{5}{|l|}{14} \\
\hline 15 & $4.29, \mathrm{t}$ & 58.5 & & $17 \mathrm{~b}$ \\
\hline 16 & & 169.1 & & \\
\hline $17 \mathrm{a}$ & $1.94, \mathrm{~m}$ & 27.4 & 16 & $15,18 \mathrm{~b}$ \\
\hline $17 \mathrm{~b}$ & $2.14, \mathrm{~m}$ & & & \\
\hline $18 \mathrm{a}$ & $1.81, \mathrm{~m}$ & 22.5 & & \\
\hline $18 \mathrm{~b}$ & $1.91, \mathrm{~m}$ & & & $19 b$ \\
\hline $19 \mathrm{a}$ & $3.35, \mathrm{~m}$ & 44.7 & & \\
\hline $19 b$ & $3.42, \mathrm{~m}$ & & & \\
\hline $1^{\prime}$ & $10.82, \mathrm{~d}(2.4)$ & & $3^{\prime}, 4^{\prime}, 9^{\prime}$ & $2^{\prime}, 8^{\prime}$ \\
\hline $2^{\prime}$ & $7.17, \mathrm{~d}(2.4)$ & 124.8 & $3^{\prime}, 4^{\prime}, 9^{\prime}$ & $1^{\prime}, 12 \mathrm{a}^{\prime}$ \\
\hline $3^{\prime}$ & & 109.6 & & \\
\hline $4^{\prime}$ & & 127.1 & & \\
\hline $5^{\prime}$ & $7.62, \mathrm{~d},(1.8)$ & 114.6 & $3,3^{\prime}, 7^{\prime}, 9^{\prime}$ & $2,5,12 \mathrm{a}$ \\
\hline $6^{\prime}$ & & 134.8 & & \\
\hline $7^{\prime}$ & $7.03, \mathrm{dd},(8.5,1.8)$ & 119.2 & $3,5^{\prime}, 9^{\prime}$ & $2,12 \mathrm{a}$ \\
\hline $8^{\prime}$ & $7.22, \mathrm{~d},(8.5)$ & 110.4 & $4^{\prime}, 6^{\prime}$ & $1^{\prime}$ \\
\hline $9^{\prime}$ & & 135.9 & & \\
\hline $10^{\prime}$ & $7.70, \mathrm{~s}$ & & $13^{\prime}$ & $11^{\prime}$ \\
\hline $11^{\prime}$ & $4.30, \mathrm{~m}$ & 55.1 & & $10^{\prime}, 15^{\prime}$ \\
\hline $12^{\prime} \mathrm{a}$ & $3.08, \mathrm{~m}$ & 25.4 & $3^{\prime}$ & \\
\hline $12^{\prime} \mathrm{b}$ & $3.21, \mathrm{~m}$ & & $13^{\prime}$ & \\
\hline $13^{\prime}$ & & 166.0 & & \\
\hline \multicolumn{5}{|l|}{$14^{\prime}$} \\
\hline $15^{\prime}$ & $4.08, \mathrm{~m}$ & 58.8 & & $11^{\prime}, 17^{\prime} \mathrm{b}$ \\
\hline $16^{\prime}$ & & 165.6 & & \\
\hline $17^{\prime} \mathrm{a}$ & $1.44, \mathrm{~m}$ & 27.7 & & \\
\hline $17^{\prime} \mathrm{b}$ & $2.01, \mathrm{~m}$ & & & \\
\hline $18 \mathrm{a}$ & $1.61, \mathrm{~m}$ & 21.9 & & \\
\hline $18^{\prime} \mathrm{b}$ & $1.69, \mathrm{~m}$ & & $13^{\prime}$ & \\
\hline $19^{\prime} \mathrm{a}$ & $3.28, \mathrm{~m}$ & 44.7 & & \\
\hline 19 'b & $3.40, \mathrm{~m}$ & & & \\
\hline
\end{tabular}

The ${ }^{1} \mathrm{H}$ NMR spectrum of the compound was well resolved and showed 28 resonances. Eight resonances were observed in the aromatic region, with two different benzene ring systems assigned from analysis of proton coupling constants and COSY spectra. Five additional proton spin systems ( $\mathrm{CHNH}$, two $\mathrm{CH}_{2} \mathrm{CH}$ and two $\mathrm{CH}_{2} \mathrm{CH}_{2} \mathrm{CH}_{2} \mathrm{CH}$ ) could be assigned on the basis of 
${ }^{1} \mathrm{H}$ and COSY spectral analysis. Correlations observed in the HSQC and HMBC spectra indicated that these proton spin systems were part of tryptophan/proline derived diketopiperazine moieties and were almost identical to those present in naseseazine B. This suggested that naseseazine $\mathrm{C}$ was a diketopiperazine dimer closely related to $\mathbf{2}$. The ortho coupled aromatic proton at $\delta_{\mathrm{H}} 7.22\left(\mathrm{H}-8^{\prime}\right)$ correlated to an upfield aromatic carbon resonating at $\delta_{\mathrm{C}} 110.4$ in the HSQC spectrum suggesting that this carbon was ortho to an electronegative substituent which indicated that it could be assigned to C-8'. Raju and co-workers reported a similar chemical shift $\left(\delta_{\mathrm{C}} 110.3\right)$ for the carbon at position $8^{\prime}$ of naseseazine B (2). ${ }^{1}$ However, since H-8' was ortho to a protonated aromatic carbon, naseseazine C (5) was likely to be a regio-isomer of 2. HMBC correlations from the two meta coupled aromatic protons, H-5' and H-7' and the indole $\mathrm{NH}$ proton to a quaternary carbon at $\delta_{\mathrm{C}} 135.9\left(\mathrm{C}-9^{\prime}\right)$ further indicated that the indole was substituted at C-6' (Figure 2). This was clearly different to the substitution pattern found in naseseazine B, suggesting that 5 had a C-3/C-6' linkage between the two subunits. HMBC correlations from H-5' and H-7' to a carbon at $\delta_{\mathrm{C}} 59.7$ (C-3) confirmed this assignment (Figure 2). By comparison structure 4 proposed by Xiong and co-workers showed intense ${ }^{4} J_{\mathrm{CH}} \mathrm{HMBC}$ correlations from $\delta_{\mathrm{H}} 7.64\left(\mathrm{H}-8^{\prime}\right.$, meta coupled doublet) to $\delta_{\mathrm{c}} 109.6\left(\mathrm{C}-3^{\prime}\right)$ and from $\delta_{\mathrm{H}} 7.12$ (H-6', ortho, meta coupled doublet of doublets) to C-8a' and no ${ }^{3} J_{\mathrm{CH}}$ correlations from $\mathrm{H}-6$ ' or $\mathrm{H}-8^{\prime}$ to $\mathrm{C}-4^{\prime}{ }^{3}$ These correlations are highly unlikely in an indole system since ${ }^{4} J_{\mathrm{CH}}$ couplings are typically small $(<1 \mathrm{~Hz})$ while ${ }^{3} J_{\mathrm{CH}}$ couplings are typically large $(>8 \mathrm{~Hz})$. The $\mathrm{HMBC}$ correlation from $\delta_{\mathrm{H}} 7.64$ to $\delta_{\mathrm{c}} 109.6$ is really only plausible if this proton is attached to $\mathrm{C}^{-} 5^{\prime}$ and since this proton is a meta coupled doublet, the carbon ortho to it (C-6') must be the point of attachment to subunit A (Figure 2). Therefore, the structure 4, presented by Xiong and co-workers wrongly assigned a C-3/C-7' linkage as shown in Figure 2 since their NMR data clearly supported a molecule (5) with a C-3/C-6' linkage.
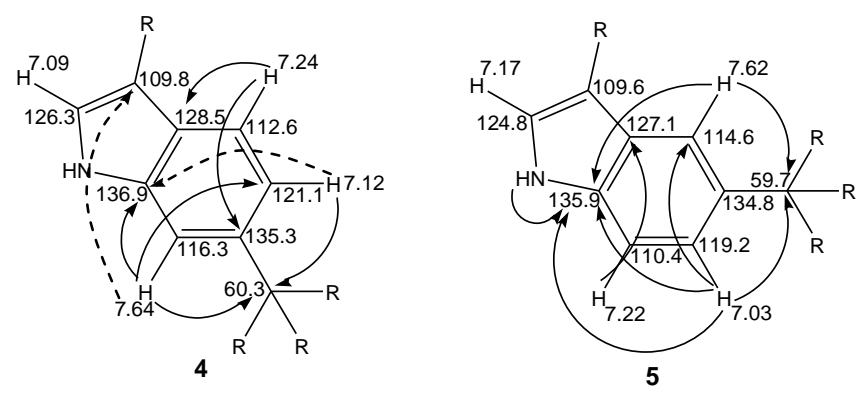

Figure 2. Key HMBC correlations observed for the indole fragments of 4 (600 $\mathrm{MHz}$ in MeOH-d 4 ) and 5 (800 MHz in DMSO-d ${ }_{6}$ ). (Dashed arrows represent ${ }^{4} \mathrm{~J}_{\mathrm{CH}}$ correlations.) 
Correlations observed in a ROESY experiment were used to assign the relative configuration of the six stereogenic centres in 5 (Figure 3). ROESY correlations between $\mathrm{H}-2\left(\delta_{\mathrm{H}} 5.62\right)$ and the aromatic protons $\mathrm{H}-5^{\prime}\left(\delta_{\mathrm{H}} 7.62\right)$ and $\mathrm{H}-7^{\prime}\left(\delta_{\mathrm{H}} 7.03\right)$ clearly showed that $\mathrm{H}-2$ and the indole in subunit B were cis to each other. Further correlations from $\mathrm{H}-2$ to $\mathrm{H}-12 \mathrm{a}\left(\delta_{\mathrm{H}} 2.96\right)$, and from $\mathrm{H}-12 \mathrm{a}$ to $\mathrm{H}-5^{\prime}$ and $\mathrm{H}-7^{\prime}$, indicated that $\mathrm{H}-12 \mathrm{a}$ was also on the $\beta$ face of subunit A. The H-11 $\left(\delta_{\mathrm{H}}\right.$ 4.16) resonance at showed ROESY correlations to $\mathrm{H}-12 \mathrm{~b}\left(\delta_{\mathrm{H}} 3.04\right)$ and $\mathrm{H}-15\left(\delta_{\mathrm{H}} 4.29\right)$ indicating that these three protons were on the $\alpha$-face of subunit A (Figure 3). Protons at H-11' $\left(\delta_{\mathrm{H}} 4.27\right)$ and $\mathrm{H}-15^{\prime}\left(\delta_{\mathrm{H}} 4.06\right)$ also had a mutual ROESY correlation indicating that they were on the same face of the molecule (Figure 3). Based upon this evidence naseseazine $\mathrm{C}$ was assigned as the $\mathrm{C}-3 / \mathrm{C}-6$ ' epimer of the synthetic compound iso-naseseazine $\mathrm{B}(\mathbf{3})$. The reported structure of 4 was proposed to differ from 2 by epimerisation at C-2 and C-3 based upon the same ROESY evidence outlined above, however, because the linkage between the two subunits was missassigned the overall structure was incorrect.

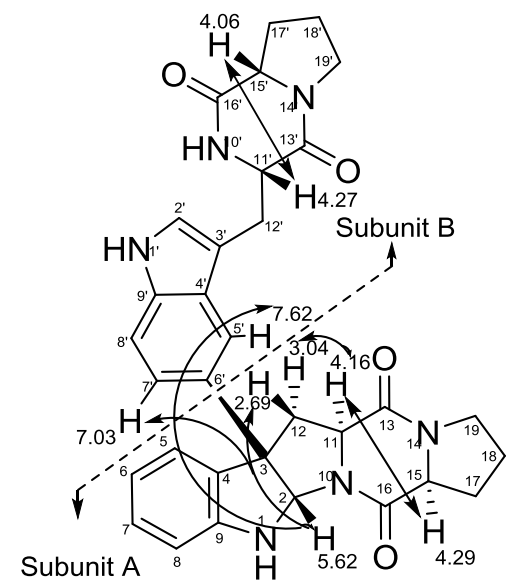

Figure 3. Relative configuration for 5 based on observed ROESY correlations

The revised structure of naseseazine $C(\mathbf{5})$ belongs to a rare class of dimeric 2,5diketopiperazines. The naseseazines along with pestalazines and asperazine are the only known examples of such dimeric diketopiperazines from a natural source and to the best of our knowledge 5 is the first natural product with a C-6 $/$ C-3 linkage. Many diketopiperazine dimers have potent biological activities including antibacterial, anti-fungal, anti-viral, anti-bloodclotting, and anti-tumour functions. ${ }^{5}$ Xiong and co-workers ${ }^{3}$ reported minor anti-fungal activity against fluconazole-resistant $C$. albicans for $\mathbf{4}$, but no other biological activity was observed in their assays. In bioassays performed by Raju and co-workers, ${ }^{1}$ no anti-microbial activity could be determined for $\mathbf{1}$ and $\mathbf{2}$ against E. coli, S. aureus, B. subtilis and C. albicans. Neither was any activity detected against cancer cell lines of gastric adenocarcinoma, neuroblastoma, erythroleukemia, or colorectal adenocarcinoma. ${ }^{1}$ 
Many diketopiperazines contain indole units derived from tryptophan. Such functional groups have often been associated with anti-plasmodial activity; in particular bis-indole alkaloids with a molecular weight higher than $400 \mathrm{~g} / \mathrm{mol}$ have shown strong anti-malarial activity. ${ }^{6}$ Also, Brevicompanine $\mathrm{B}$ was reported to be active against the Plasmodium falciparum $\left(\mathrm{IC}_{50} 95\right.$ $\mathrm{mM}) ;^{7}$ this compound has the same substructure as naseseazine A. Thus biological assessment targeting the malaria parasite was pursued. Compounds 1, 2 and $\mathbf{5}$ were tested in three separate experiments for their activity to inhibit the growth of a chloroquine sensitive strain of Plasmodium falciparum (3D7) as described by Duffy and Avery. ${ }^{8}$ Neither $\mathbf{1}$ nor $\mathbf{2}$ showed activity against the malaria parasite at a dose of $20 \mu \mathrm{M}$. Naseseazine $\mathrm{C}(\mathbf{5})$, however, displayed moderate anti-plasmodial activity with an average $\mathrm{IC}_{50}$ value of $3.52 \mu \mathrm{M}( \pm 1.2)$. This suggests that the C-6/C-3 linkage between the two subunits and/or the change in regiochemistry in subunit A could be responsible for the enhanced bioactivity.

In conclusion, three 2,5-diketopiperazines were isolated from a marine sediment derived Streptomyces strain. Naseseazines A and B were previously described, while naseseazine C displayed a new C-6'/C-3 linkage; this is the first time that this family of compounds was investigated for anti-plasmodial activity. These results showed that marine actinomycetes can be a potential source for anti-plasmodial compounds and studies are currently ongoing to discover other Plasmodium inhibiting natural products.

\subsection{Experimental section}

\subsubsection{General Experimental Procedures}

Extracts were dried with a rotary evaporator (Christ Rotational Vacuum Concentrator - RVC 2-25 CD plus). Optical rotations were measured on a JASCO P-1020 polarimeter for Naseseazine A, B and C. For the new compound naseseazine C, a UV spectrum was obtained on a Shimadzu UV-1800 UV/vis spectrophotometer. NMR data for naseseazine A and B were obtained on a Varian $600 \mathrm{MHz}$ Unity INOVA spectrometer. All NMR spectra for naseseazine C were recorded at $25{ }^{\circ} \mathrm{C}$ on a Bruker BioSpin $\mathrm{GmbH} 800 \mathrm{MHz}$ spectrometer. The ${ }^{1} \mathrm{H}$ and ${ }^{13} \mathrm{C}$ chemical shifts were referenced to the DMSO- $d_{6}$ solvent peak at $\delta_{\mathrm{H}} 2.50$ and $\delta_{\mathrm{C}} 39.5$, respectively. Standard parameters were used for 2D NMR spectra obtained, including gCOSY, gHSQC, gHMBC and ROESY. HSESI-QTOFMS were recorded on a 6530 Accurate Mass QTOF (Agilent Technologies) workstation using positive electrospray ionization, with mobile phase ACN/H2O containing 0.1\% formic acid. A LaChrom Merck Hitachi L7100 series pump equipped with a Hitachi L7450 PDA detector was used for HPLC separations. Prior to HPLC separation the microbial crude extract was adsorbed Alltech Davisil 35- $75 \mu \mathrm{m} 150 \AA \mathrm{C}_{18}$. All 
separations were performed with a Thermo Hypersil-Keystone Betasil $5 \mu \mathrm{m} \AA \mathrm{C}_{18}$ HPLC column $(21.2 \mathrm{~mm} \times 150 \mathrm{~mm})$. EtOAc used for extractions was Honeywell HPLC grade. All solvents used for HPLC, UV, and $[\alpha]_{\mathrm{D}}$ were Scharlau HPLC grade. For MS, Fisher Scientific LC/MS grade acetonitrile was used and $\mathrm{H}_{2} \mathrm{O}$ was Millipore Milli-Q PF filtered.

\subsubsection{Microbial isolation}

Streptomyces species (USC-636) was isolated from near shore marine sediments samples collected in Maroochydore, Sunshine Coast, QLD, Australia. Starch-casein agar supplemented with antifungal agents cycloheximide and nystatin $(75 \mathrm{ppm})$ and nalidixic acid $(25 \mathrm{ppm})$ to prevent the growth of non-actinomycete taxa was used for the isolation. ${ }^{9,}{ }^{10}$ The isolate was identified via the PCR method using the QIAGEN HotStarTaq ${ }^{\circledR}$ Multiplex PCR Kit and the primers B27F and 1492R. ${ }^{11}$ The PCR products were sequenced at Macrogen Inc. (South Korea, http://www.macrogen.com/). 16S rRNA sequences were subsequently deposited in the GenBank (http://www.ncbi.nlm.nih.gov/genbank/) (GenBank accession number KX379154). A phylogenetic tree was constructed using these sequences with bootstrap analysis using 1000 data re-samplings. ${ }^{12,13}$ Sequences were also checked for taxonomic classifications using the Greengenes 'classifier' (Figure 4). ${ }^{14}$

Subsequent Scanning Electron Microscopy imaging conducted at Central Analytical Research Facility operated by the Institute for Future Environments of the Queensland University of Technology, Brisbane, Australia revealed the spiral spore chains of the isolate (Figure 5).

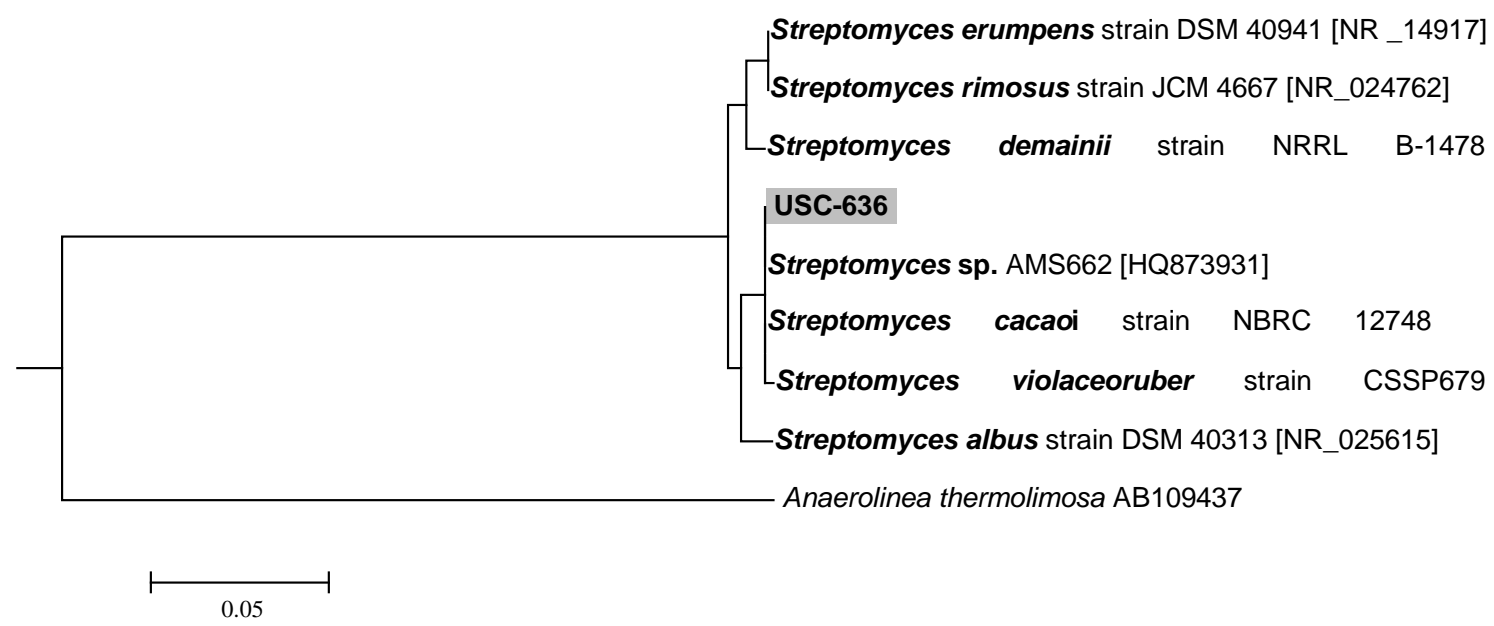

Figure 4. Phylogenetic tree of the Streptomyces isolate (USC-636) in relation to its nearest relatives (Anaerolinea thermolimosa $\mathrm{AB109437}$ was used as an out-group sequence to root the tree). 


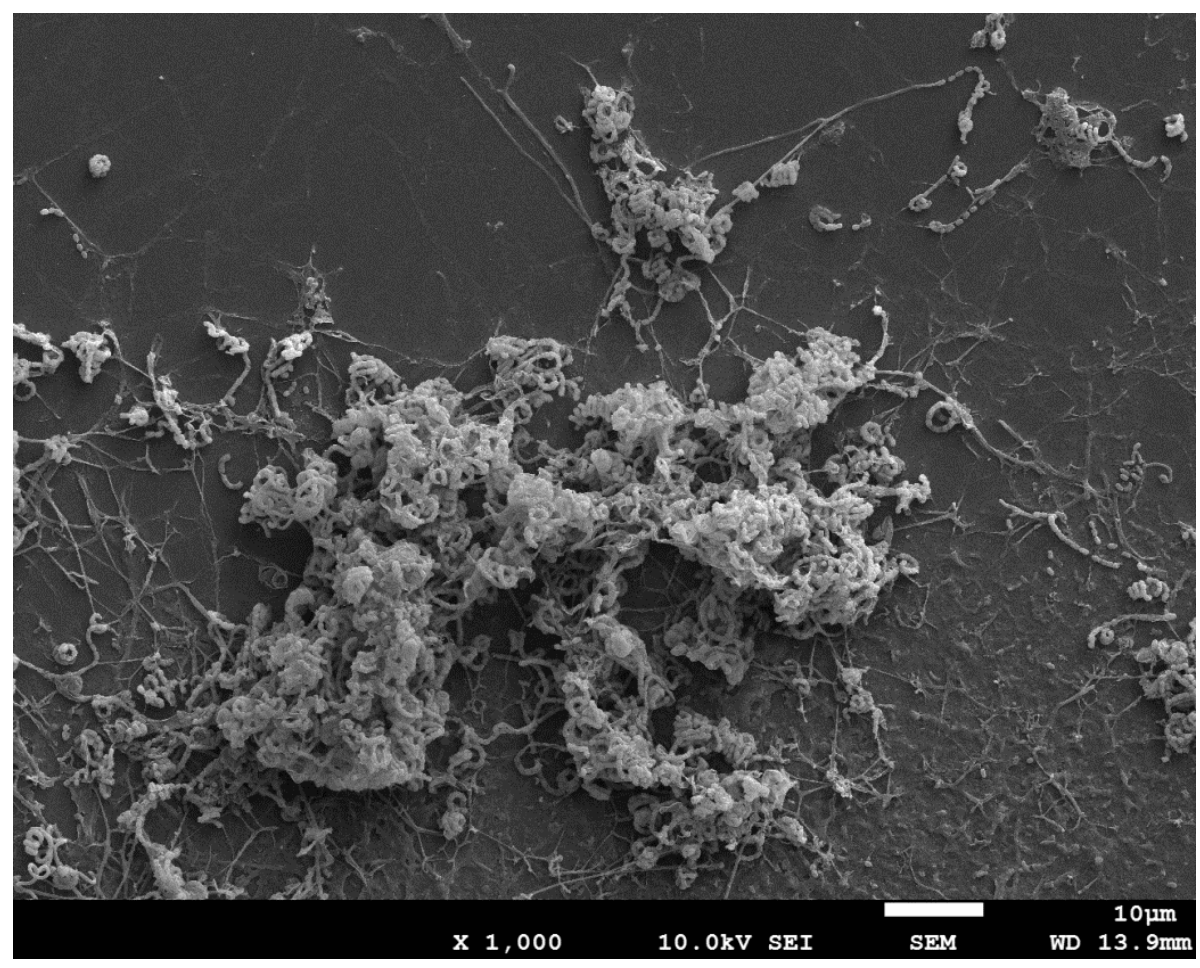

Figure 5. Electron micrograph of the Streptomyces isolate (USC-636) (bar indicates $10 \mu \mathrm{m}$ )

\subsubsection{Extraction and Isolation}

Fifty-two agar plates containing microbial cultures were blended with a conventional kitchen blender and repeatedly extracted in ethyl acetate (EtOAc) in a sonic bath. The extract was dried with a rotary evaporator under vacuum. The residue $\left(373 \mathrm{mg}\right.$ ) was adsorbed onto $\mathrm{C}_{18}$ silica gel (1:1) and loaded into a refillable HPLC guard column (10 $\mathrm{mm} \times 20 \mathrm{~mm})$ attached to the HPLC system and eluted with a linear solvent gradient from $90 \% \mathrm{H}_{2} \mathrm{O} / 10 \% \mathrm{MeOH}$ to $100 \% \mathrm{MeOH}$ at a flow rate of $9 \mathrm{~mL} / \mathrm{min}$ over 60 minutes. Sixty one-minute fractions were collected. UV spectra were obtained at $210 \mathrm{~nm}, 254 \mathrm{~nm}, 280 \mathrm{~nm}, 320 \mathrm{~nm}$ and $380 \mathrm{~nm}$. Fractions containing similar signals were combined. The naseseazines eluted in four fractions around $37 \% \mathrm{MeOH}$ as a mixture of compounds. These fractions were recombined and separated further using refined solvent gradients ( $10 \%$ to $50 \% \mathrm{MeOH}$ and $10 \%$ to $40 \% \mathrm{MeOH})$ until naseseazine $\mathrm{A}(0.1 \mathrm{mg})$, $\mathrm{B}(0.03 \mathrm{mg})$ and $\mathrm{C}(0.05 \mathrm{mg})$ were obtained as pure fractions. 


\subsection{Acknowledgments}

The authors would like to thank C. Brinkmann (Genecology Research Centre, University of the Sunshine Coast, Australia) for molecular identification of the isolate, Dr. Ken Wasmund (Division of Microbial Ecology, Department of Microbiology and Ecosystem Science, University of Vienna, Austria) for the construction of the phylogenetic tree and Ms. Rachel Hancock (Central Analytical Research Facility, the Institute for Future Environments of Queensland University of Technology, Brisbane, Australia) for support with the SEM analysis. Biological profiling was supported by an Australian Research Council grant LP120200557 awarded to V. M. Avery. We thank and acknowledge the Australian Red Cross Blood Bank for provision of the red blood cells essential for this research. L. Buedenbender acknowledges the provision of an Australian Postgraduate Award and Griffith University International Postgraduate Research Scholarship. The research was funded in parts by the Griffith School of Environment.

\subsection{References}

(1) Raju, R.; Piggott, A. M.; Conte, M.; Aalbersberg, W. G. L.; Feussner, K.; Capon, R. J. Org. Lett. 2009, 11, 3862-3865.

(2) Kim, J.; Movassaghi, M. J. Am. Chem. Soc. 2011, 133, 14940-14943.

(3) Xiong, Z.-Q.; Liu, Q.-X.; Pan, Z.-L.; Zhao, N.; Feng, Z.-X.; Wang, Y. Arch. Microbiol. 2015, 197, 299-309.

(4) Naseseazine $\mathrm{C}(5)$ : yellow gum $\left(0.05 \mathrm{mg},[\alpha]_{\mathrm{D}}-18.6\right.$ (c $\left.0.05 \mathrm{mg}, \mathrm{MeOH}\right) . \mathrm{UV}(\mathrm{MeOH})$ $\lambda_{\max }(\log \varepsilon) 205 \mathrm{~nm}(2.25), 225 \mathrm{~nm}(2.16), 286 \mathrm{~nm}(1.46), 293 \mathrm{~nm}(1.45) .{ }^{1} \mathrm{H}$ and ${ }^{13} \mathrm{C}$ NMR (see Table 1); (+)-HSESI-QTOFMS m/z 565.2576 (calculated for $\mathrm{C}_{32} \mathrm{H}_{32} \mathrm{~N}_{6} \mathrm{O}_{4}[\mathrm{M}+\mathrm{H}]$ : 565.2558, $\Delta+3.22 \mathrm{ppm})$.

(5) Martins, M. B.; Carvalho, I. Tetrahedron 2007, 63, 9923-9932.

(6) Frederich, M.; Tits, M.; Angenot, L. Trans. R. Soc. Trop. Med. Hyg. 2008, 102, 11-19.

(7) Sprogøe, K.; Manniche, S.; Larsen, T. O.; Christophersen, C. Tetrahedron 2005, 61, 8718-8721.

(8) Duffy, S.; Avery, V. M. Am. J. Trop. Med. Hyg. 2012, 86, 84-92.

(9) Küster, E.; Williams, S. T. Nature 1964, 202, 928-929.

(10) Wakisaka, Y.; Kawamura, Y.; Yasuda, Y.; Koizumi, K.; Nishimoto, Y. J Antibiot. 1982, $35,822-836$.

(11) Kullen, M. J.; Sanozky-Dawes, R. B.; Crowell, D. C.; Klaenhammer, T.R. J. Appl. Microbiol. 2000, 89, 511-516.

(12) Saitou, N.; Nei, M. Mol. Biol. Evol. 1987, 4, 406-425. 
(13) Ludwig, W.; Strunk, O.; Westram, R.; Richter, L.; Meier, H.; Yadhukumar, B. A.; Lai, T.; Steppi, S.; Jobb, G.; Föster, W.; Brettske, I.; Gerber, S.; Ginhart, A. W.; Gross, O.; Grumann, S.; Hermann, S.; Jost, R.; König, A.; Liss, T.; Lüssmann, R.; May, M.; Nonhoff, B.; Reichel, B.; Strehlow, R.; Stamatakis, A.; Stuckmann, N.; Vilbig, A.; Lenke, M.; Ludwig, T.; Bode, A.; Schleifer, K. H. Nucleic Acids Res. 2004, 32, 13631371.

(14) DeSantis, T. Z.; Hugenholtz, P.; Larsen, N.; Rojas, M.; Brodie, E. L.; Keller, K..; Huber, T.; Dalevi, D.; Hu, P.; Andersen, G. L. Appl. Environ. Microbiol. 2006, 72, 5069-5072 


\section{CHAPTER 7 - Conclusion and future outlook}

The work of this thesis was aimed to integrate and explore different biodiscovery approaches to maximise natural product discovery from marine actinomycetes. The findings presented in this thesis highlight the potential of marine actinomycete in natural product drug discovery. Natural products present the most successful ecologically adapted metabolites that have evolved to interact with specific biological targets and therefore make effective leads for drug discovery. Microbial natural products, specifically from actinomycetes, offer an unmatched structural diversity and offer a sustainable source of supply. In the past numerous actinomycete metabolites have made their way as drugs to the pharmaceutical market. However, the isolation and elucidation of natural products is time consuming and rediscovery of already known compounds is a major challenge. Consequently, interdisciplinary approaches that connect molecular and microbiology, bioinformatics, metabolomics and analytical chemistry are needed for successful natural product drug discovery.

\section{Untapped ecological niche: Ascidian - actinomycete associations}

In order to expand chemical diversity of secondary metabolites there has been an urge to isolate new microbes from new ecological niches, particularly the marine environment. Oceans cover $70 \%$ of the earth's surface and provide a vast, untapped and diverse resource for bioprospecting. As seawater is low in nutrients many marine microorganisms live in associations with marine invertebrates such as sponges and ascidians, which have historically been excellent sources for biologically active metabolites. Increasing evidence shows that many natural products isolated from marine invertebrates are actually biosynthesised by their microbial symbionts and recent natural product discovery programs targeting marine invertebrate associates have revealed an array of previously undiscovered metabolites.

In chapter 2, the actinomycete diversity associated with three different Australian ascidian species was examined based on culture-dependent and independent techniques. A broad diversity of 120 natural product producing actinomycetes, mainly from the genera Streptomyces and Micromonospora, were isolated. Interestingly, very little overlap of actinomycete associates between the three ascidians was observed. This suggested that actinomycete associations with ascidians may be species-specific. One Streptomyces sp. strain isolate had a $99.2 \%$ identical $16 \mathrm{~S}$ rRNA sequence to an isolate that was previously derived from marine sediments, it was concluded that actinomycetes are most likely selectively acquired by the ascidian host from the environment. Further studies will be needed to confirm this hypothesis. A GNPS molecular ion 
network was established in order to evaluate the metabolite potential of ascidian-associated actinomycetes and to identify compounds of the ascidian host that are produced by a microbial isolate. Overlap between ascidian hosts and associated Streptomyces, Micromonospora, Streptosporangium and Nocardia isolates was observed for several ions; while Rhodoccocus did not appear to play a role in the ascidians' metabolomes. The actinomycete MS metabolite profiles, specifically for Streptomyces and Micromonospora isolates, were highly diverse and thus present a good target for natural product drug discovery.

\section{Tackling the issue of rediscovery and dereplication}

Rediscovery of already known natural products poses a great challenge to natural product drug discovery. Therefore, it is important to incorporate effective dereplication protocols early in natural product isolation efforts. In Chapter 3, a new NMR-based metabolomics approach for microbial strain prioritisation was presented. HSQC-TOCSY NMR spectra were used to characterise polyketide and peptide diversity in microbial crude extracts. These spectra were subsequently analysed in combination with anti-plasmodial activity data to systematically prioritise microbial strains for natural product discovery that have higher potential to produce bioactive metabolites. NMR is an extremely powerful analytical tool and, in contrast to MS, it provides structural information of compounds in the microbial extracts and can be used to target specific groups of natural products. Moreover, the use of two-dimensional NMR experiments offered increased chemical shift resolution by spreading the structural information onto two dimensions and thus permitted assessment of unfractionated extracts. The HSQC-TOCSY NMR experiment was proven to be much more sensitive compared to HMBC NMR experiments, and HSQC-TOCSY spectra could be acquired in about 30 minutes.

The application of HSQC-TOCSY NMR experiments for the purpose of microbial strain selection (Chapter 3) as well as for assessment of different culture conditions (Chapter 4) proved to be very valuable. Out of 119 microbial isolates, Streptomyces sp. (USC-16018) was selected based on its anti-plasmodial activity and diverse HSQC-TOCSY NMR peaks associated with secondary metabolites, in particular polyketides, which were subsequently isolated. This was the first time that HSQC-TOCSY spectra were utilised in this manner, and compared to MS or 1D NMR profiling techniques, the new approach readily revealed the presence and abundance of polyketides in the extracts. 


\section{Marine actinomycetes as a source of new anti-plasmodial compounds}

The HSQC-TOCSY NMR spectrum of the ascidian-associated Streptomyces sp. USC16018 indicated presence of a broad diversity of polyketide-associated cross-peaks. Subsequent large scale fermentation and preparative HPLC resulted in the purification of a new ansamycin polyketide, herbimycin G, as well as the known natural products, elaiophylin, Cyclo-L-Pro-LLeu, Cyclo-L-Pro-L-Phe, Cyclo-L-Pro-L-Val and Cyclo-L-Pro-L-Tyr. Ansamycin polyketides have previously been identified from terrestrial and marine actinomycetes, with geldanamycin proceeding to advanced clinical trials as an anti-tumour drug. Many ansamycin compounds act as Hsp90 inhibitors. When the Plasmodium parasite changes host, heat shock proteins including Hsp90 are highly expressed in the parasite and thus Hsp90 inhibitors have been proposed as a good drug target for anti-malarial drugs. Therefore, herbimycin $\mathrm{G}$ was tested against a chloroquine-sensitive and chloroquine-resistant $P$. falciparum strain and caused significant inhibition (> $75 \%$ ) of both parasite strains (Chapter 5).

A new dimeric diketopiperazine was isolated from the marine sediment derived Streptomyces sp. USC-636. Naseseazine C was a new regio-isomer of naseseazine B, which was previously isolated from a marine Fijian sediment-derived actinomycetes species. Isonaseseazine B isolated from a Yellow Sea sediment-derived Streptomyces sp. published in the literature was proposed to be a stereoisomer of naseseazine B. However, when comparing the spectroscopic data published with our experimental data, we found that they were identical. However, indisputable evidence of the NMR spectra indicated a new C-6'/C-3 linkage between the two diketopiperazine subunits of the compound, leading to the conclusion that the reported structure in the literature was in fact incorrect. In Chapter 6, the full isolation and structure elucidation of the new compounds was described. These findings were also published as a peerreview article in the journal Tetrahedron Letters. We also screened naseseazines A - C against $P$. falciparum and found that naseseazine $\mathrm{C}$ was active, while $\mathrm{A}$ and $\mathrm{B}$ were not. This difference in activity may be associated with the different C-6'/C-3 linkage.

\section{Remarks and future directions}

The aims of the thesis were successfully met and a library of 120 ascidian-associated actinomycetes was established. Based on the newly established strain prioritisation approach, Streptomyces sp. USC-16018 was selected and a new anti-plasmodial polyketide was isolated. Overall, this thesis highlights the immense chemical diversity of actinomycetes associated with marine invertebrates, as well as the importance of sophisticated dereplication approaches and provides an alternative new solution for strain selection that has led to the discovery of new 
bioactive natural products with potential as new drug leads. Another 14 actinomycete isolates had interesting HSQC-TOCSY profiles and exhibited above $80 \%$ inhibition against $P$. falciparum. Large scale fermentation of the bioactive strains and natural product isolation efforts will be pursued in the future. As observed in Chapter 3, co-cultivation often results in enhanced chemical diversity of the extracts and de novo synthesis of secondary metabolites. As such, co-cultivations will be implemented in the fermentation process of the selected actinomycete strains.

Actinomycetes are prolific producers of bioactive metabolites. However, it was noted that even though new anti-plasmodial compounds were isolated, the core structures of both naseseazine $\mathrm{C}$ and herbimycin $\mathrm{G}$ were previously reported in actinomycete extracts. The culture-independent approach taken to uncover the microbial diversity of the ascidians revealed that actinomycetes only account for less than $2 \%$ of the microbiome and there remains a huge diversity of unculturable and thus untapped microbial families. Less effort has been made to isolate unculturable microorganisms and thus still represent an untapped source for biodiscovery. In earlier stages of this $\mathrm{PhD}$ project, a device for in situ isolation of marine microorganisms was designed, but due to time constrains its implementation could not yet be realised (Appendix V). The so-called iChip contains numerous miniature diffusion chambers in which individual cells of environmental bacteria can be isolated simultaneously. This approach has successfully been used for isolation of soil bacteria and has resulted in the isolation of a novel bacterium that produced the novel antibiotic teixobactin. For the future, it is envisaged that the modified version of the iChip will be implanted into living marine invertebrate tissue in order to isolate new symbiotic bacteria in situ that will yield novel natural product scaffolds. 


\section{APPENDIX I - for Chapter 2}

Table IA - Ascidian-associated actinomycete collection

\begin{tabular}{|c|c|c|c|c|c|}
\hline Isolate-ID & OTU & Ascidian host & $\begin{array}{c}\text { Isolation } \\
\text { media }\end{array}$ & $\begin{array}{c}\text { 16S rRNA } \\
\text { identification }\end{array}$ & $\begin{array}{c}\text { GenBank } \\
\text { accession \# }\end{array}$ \\
\hline USC16000 & denovo32 & S. rubra & SCA & Streptomyces sp. & MF773756 \\
\hline USC16001 & denovo11 & S. rubra & CS & Streptomyces sp. & MF773757 \\
\hline USC16002 & denovo69 & S. rubra & CS & Streptomyces sp. & MF773758 \\
\hline USC16003 & denovo19 & S. rubra & $\mathrm{CS}$ & Streptomyces sp. & MF773759 \\
\hline USC16004 & denovo49 & S. rubra & $\mathrm{CS}$ & Streptomyces sp. & MF773760 \\
\hline USC16005 & denovo43 & S. rubra & $\mathrm{CS}$ & Streptomyces sp. & MF773761 \\
\hline USC16006 & denovo0 & S. rubra & $\mathrm{CS}$ & Streptomyces sp. & MF773762 \\
\hline USC16007 & denovo28 & S. rubra & $\mathrm{CS}$ & Streptomyces sp. & MF773763 \\
\hline USC16008 & denovo68 & S. rubra & $\mathrm{CS}$ & Streptomyces sp. & MF773764 \\
\hline USC16009 & denovo57 & S. rubra & $\mathrm{CS}$ & Streptomyces sp. & MF773765 \\
\hline USC16010 & denovo38 & S. rubra & $\mathrm{CS}$ & Streptomyces sp. & MF773766 \\
\hline USC16011 & denovo10 & S. rubra & $\mathrm{CS}$ & Streptomyces sp. & MF773767 \\
\hline USC16012 & denovo11 & S. rubra & $\mathrm{CS}$ & Streptomyces sp. & MF773768 \\
\hline USC16013 & denovo24 & S. rubra & $\mathrm{CS}$ & Streptomyces sp. & MF773769 \\
\hline USC16014 & denovo34 & S. rubra & $\mathrm{CS}$ & Streptomyces sp. & MF773770 \\
\hline USC16015 & denovo4 & S. rubra & $\mathrm{CS}$ & Streptomyces sp. & MF773771 \\
\hline USC16016 & denovo 25 & S. rubra & $\mathrm{CS}$ & Streptomyces sp. & MF773772 \\
\hline USC16017 & denovo29 & S. rubra & $\mathrm{CS}$ & Streptomyces sp. & MF773773 \\
\hline USC16018 & denovo19 & S. rubra & $\mathrm{CS}$ & Streptomyces sp. & MF773774 \\
\hline USC16019 & denovo48 & S. rubra & $\mathrm{CS}$ & Streptomyces sp. & MF773775 \\
\hline USC 16020 & denovo48 & S. rubra & $\mathrm{CS}$ & Streptomyces sp. & MF773776 \\
\hline USC16021 & denovo30 & S. rubra & SCA & Streptomyces sp. & MF773777 \\
\hline USC16022 & denovo29 & A. solidium & $\mathrm{CS}$ & Streptomyces sp. & MF773778 \\
\hline USC16023 & denovo73 & A. solidium & $\mathrm{CS}$ & Streptomyces sp. & MF773779 \\
\hline USC16024 & denovo 27 & P. vasculosum & SCA & Streptomyces sp. & MF773780 \\
\hline USC16025 & denovo18 & P. vasculosum & SCA & Streptomyces sp. & MF773781 \\
\hline USC16026 & denovo63 & P. vasculosum & $\mathrm{CS}$ & Streptomyces sp. & MF773782 \\
\hline USC16027 & denovo74 & S. rubra & $\mathrm{CS}$ & Streptomyces sp. & MF773783 \\
\hline USC16028 & denovo67 & S. rubra & $\mathrm{SA}$ & Micromonospora sp. & MF773784 \\
\hline USC16029 & denovo67 & S. rubra & SCA & Micromonospora sp. & MF773785 \\
\hline USC16030 & denovo5 & S. rubra & $\mathrm{SA}$ & Micromonospora sp. & MF773786 \\
\hline USC16031 & denovo46 & S. rubra & SCA & Micromonospora sp. & MF773787 \\
\hline USC16032 & denovo67 & S. rubra & SCA & Micromonospora sp. & MF773788 \\
\hline USC16033 & denovo70 & S. rubra & $\mathrm{SCA}$ & Micromonospora sp. & MF773789 \\
\hline USC16034 & denovo67 & S. rubra & SCA & Micromonospora sp. & MF773790 \\
\hline USC16035 & denovo 23 & S. rubra & SCA & Micromonospora sp. & MF773791 \\
\hline USC16036 & denovo52 & S. rubra & SCA & Micromonospora sp. & MF773792 \\
\hline USC16037 & denovo1 & S. rubra & SCA & Micromonospora sp. & MF773793 \\
\hline USC16038 & denovo71 & S. rubra & SCA & Micromonospora sp. & MF773794 \\
\hline USC16039 & denovo16 & S. rubra & $\mathrm{SCA}$ & Micromonospora sp. & MF773795 \\
\hline USC16040 & denovo52 & S. rubra & SCA & Micromonospora sp. & MF773796 \\
\hline USC16041 & denovo12 & S. rubra & $\mathrm{CS}$ & Streptomyces sp. & MF773797 \\
\hline
\end{tabular}


Table IA - Ascidian-associated actinomycete collection (continued)

\begin{tabular}{|c|c|c|c|c|c|}
\hline Isolate-ID & OTU & Ascidian host & $\begin{array}{c}\text { Isolation } \\
\text { media }\end{array}$ & $\begin{array}{c}\text { 16S rRNA } \\
\text { identification }\end{array}$ & $\begin{array}{c}\text { GenBank } \\
\text { accession \# }\end{array}$ \\
\hline USC16042 & denovo51 & S. rubra & SA & Micromonospora sp. & MF773798 \\
\hline USC16043 & denovo61 & S. rubra & SA & Micromonospora sp. & MF773799 \\
\hline USC16044 & denovo56 & S. rubra & SA & Micromonospora sp. & MF773800 \\
\hline USC16045 & denovo70 & S. rubra & SA & Micromonospora sp. & MF773801 \\
\hline USC16046 & denovo33 & S. rubra & SA & Micromonospora sp. & MF773802 \\
\hline USC16047 & denovo37 & S. rubra & SA & Micromonospora sp. & MF773803 \\
\hline USC16048 & denovo 23 & S. rubra & SA & Micromonospora sp. & MF773804 \\
\hline USC16049 & denovo51 & S. rubra & SCA & Micromonospora sp. & MF773805 \\
\hline USC 16050 & denovo52 & S. rubra & SCA & Micromonospora sp. & MF773806 \\
\hline USC16051 & denovo37 & S. rubra & SA & Micromonospora sp. & MF773807 \\
\hline USC16052 & denovo 70 & S. rubra & SA & Micromonospora sp. & MF773808 \\
\hline USC16053 & denovo50 & S. rubra & SA & Micromonospora sp. & MF773809 \\
\hline USC16054 & denovo67 & S. rubra & SCA & Micromonospora sp. & MF773810 \\
\hline USC16055 & denovo61 & S. rubra & SCA & Micromonospora sp. & MF773811 \\
\hline USC16056 & denovo8 & S. rubra & SCA & Micromonospora sp. & MF773812 \\
\hline USC16057 & denovo 26 & S. rubra & SCA & Micromonospora sp. & MF773813 \\
\hline USC16058 & denovo67 & S. rubra & SCA & Micromonospora sp. & MF773814 \\
\hline USC16059 & denovo52 & S. rubra & SCA & Micromonospora sp. & MF773815 \\
\hline USC16060 & denovo 70 & S. rubra & SCA & Micromonospora sp. & MF773816 \\
\hline USC16061 & denovo13 & S. rubra & SCA & Micromonospora sp. & MF773817 \\
\hline USC16062 & denovo31 & S. rubra & SCA & Streptomyces sp. & MF773818 \\
\hline USC16063 & denovo47 & S. rubra & SCA & Streptomyces sp. & MF773819 \\
\hline USC16064 & denovo53 & A. solidium & SCA & Micromonospora sp. & MF773820 \\
\hline USC16065 & denovo67 & A. solidium & SCA & Micromonospora sp. & MF773821 \\
\hline USC16066 & denovo66 & A. solidium & SCA & Micromonospora sp. & MF773822 \\
\hline USC16067 & denovo 72 & A. solidium & SCA & Micromonospora sp. & MF773823 \\
\hline USC16068 & denovo36 & A. solidium & SCA & Streptomyces sp. & MF773824 \\
\hline USC16069 & denovo17 & A. solidium & SCA & Micromonospora sp. & MF773825 \\
\hline USC16070 & denovo67 & A. solidium & SCA & Micromonospora sp. & MF773826 \\
\hline USC16071 & denovo 72 & P. vasculosum & SA & Micromonospora sp. & MF773827 \\
\hline USC16072 & denovo 70 & A. solidium & SCA & Micromonospora sp. & MF773828 \\
\hline USC16073 & denovo70 & A. solidium & SCA & Micromonospora sp. & MF773829 \\
\hline USC16074 & denovo70 & A. solidium & SCA & Micromonospora sp. & MF773830 \\
\hline USC16075 & denovo 70 & A. solidium & SCA & Micromonospora sp. & MF773831 \\
\hline USC16076 & denovo41 & P. vasculosum & SCA & Micromonospora sp. & MF773832 \\
\hline USC16077 & denovo39 & P. vasculosum & SCA & Micromonospora sp. & MF773833 \\
\hline USC16078 & denovo55 & P. vasculosum & SCA & Micromonospora sp. & MF773834 \\
\hline USC16079 & denovo58 & P. vasculosum & SCA & Micromonospora sp. & MF773835 \\
\hline USC16080 & denovo 22 & P. vasculosum & SCA & Micromonospora sp. & MF773836 \\
\hline USC16081 & denovo67 & P. vasculosum & SA & Micromonospora sp. & MF773837 \\
\hline USC16082 & denovo3 & P. vasculosum & SA & Micromonospora sp. & MF773838 \\
\hline USC16083 & denovo67 & P. vasculosum & SCA & Micromonospora sp. & MF773839 \\
\hline USC16084 & denovo9 & P. vasculosum & SCA & Micromonospora sp. & MF773840 \\
\hline USC16085 & denovo 2 & P. vasculosum & SA & Micromonospora sp. & MF773841 \\
\hline USC16086 & denovo51 & P. vasculosum & SCA & Micromonospora sp. & MF773842 \\
\hline USC16087 & denovo9 & P. vasculosum & SCA & Micromonospora sp. & MF773843 \\
\hline
\end{tabular}


Table IA - Ascidian-associated actinomycete collection (continued)

\begin{tabular}{|c|c|c|c|c|c|}
\hline Isolate-ID & OTU & Ascidian host & $\begin{array}{c}\text { Isolation } \\
\text { media }\end{array}$ & $\begin{array}{c}\text { 16S rRNA } \\
\text { identification }\end{array}$ & $\begin{array}{c}\text { GenBank } \\
\text { accession \# }\end{array}$ \\
\hline USC16088 & denovo61 & P. vasculosum & $\mathrm{SA}$ & Micromonospora sp. & MF773844 \\
\hline USC16089 & denovo53 & P. vasculosum & SA & Micromonospora sp. & MF773845 \\
\hline USC16090 & denovo37 & P. vasculosum & SA & Micromonospora sp. & MF773846 \\
\hline USC16091 & denovo7 & A. solidium & $\mathrm{CS}$ & Nocardia sp. & MF773847 \\
\hline USC16092 & denovo20 & S. rubra & $\mathrm{CS}$ & Rhodoccocus sp. & MF773848 \\
\hline USC16093 & denovo7 & S. rubra & $\mathrm{CS}$ & Nocardia sp. & MF773849 \\
\hline USC16094 & denovo14 & S. rubra & $\mathrm{CS}$ & Rhodoccocus sp. & MF773850 \\
\hline USC16095 & denovo42 & S. rubra & SCA & Nocardia sp. & MF773851 \\
\hline USC16096 & denovo45 & S. rubra & $\mathrm{SCA}$ & Nocardia sp. & MF773852 \\
\hline USC16097 & denovo64 & S. rubra & SCA & Nocardia sp. & MF773853 \\
\hline USC16098 & denovo75 & A. solidium & SCA & Streptomyces sp. & MF773854 \\
\hline USC16099 & denovo76 & A. solidium & SCA & Streptomyces sp. & MF773855 \\
\hline USC16100 & denovo6 & S. rubra & $\mathrm{CS}$ & Streptomyces sp. & MF773856 \\
\hline USC16101 & denovo21 & S. rubra & SA & Streptomyces sp. & MF773857 \\
\hline USC16102 & denovo52 & S. rubra & SA & Micromonospora sp. & MF773858 \\
\hline USC16103 & denovo35 & S. rubra & SCA & Micromonospora sp. & MF773859 \\
\hline USC16104 & denovo70 & S. rubra & SCA & Micromonospora sp. & MF773860 \\
\hline USC16105 & denovo40 & S. rubra & SCA & Micromonospora sp. & MF773861 \\
\hline USC16106 & denovo61 & A. solidium & $\mathrm{SCA}$ & Micromonospora sp. & MF773862 \\
\hline USC16107 & denovo54 & P. vasculosum & SCA & Micromonospora sp. & MF773863 \\
\hline USC16108 & denovo59 & S. rubra & SCA & Micromonospora sp. & MF773864 \\
\hline USC16109 & denovo61 & A. solidium & SCA & Micromonospora sp. & MF773865 \\
\hline USC16110 & denovo38 & S. rubra & $\mathrm{CS}$ & Streptomyces sp. & MF773866 \\
\hline USC16111 & denovo62 & S. rubra & SCA & Micromonospora sp. & MF773867 \\
\hline USC16112 & denovo70 & S. rubra & $\mathrm{CS}$ & Micromonospora sp. & MF773868 \\
\hline USC16113 & denovo60 & P. vasculosum & MA & Streptomyces sp. & MF773869 \\
\hline USC16114 & denovo67 & S. rubra & MA & Micromonospora sp. & MF773870 \\
\hline USC16115 & denovo44 & S. rubra & MA & Streptomyces sp. & MF773871 \\
\hline USC16117 & denovo4 & S. rubra & MA & Streptomyces sp. & MF773872 \\
\hline USC16118 & denovo4 & S. rubra & MA & Streptomyces sp. & MF773873 \\
\hline USC16119 & denovo77 & S. rubra & MA & Streptosporangium sp. & MF773874 \\
\hline USC16120 & denovo7 & A. solidium & $\mathrm{NH}$ & Nocardia sp. & MF773875 \\
\hline
\end{tabular}


Appendix Ia: Ascidian-associated actinomycete library (USCUniversity of Sunshine Coast)

\section{Streptomyces sp. isolates:}

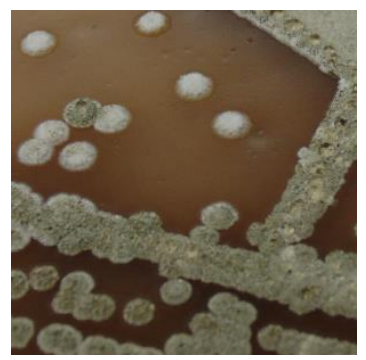

USC16000

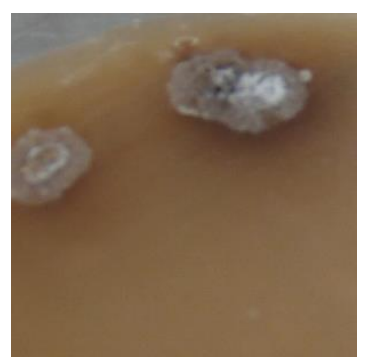

USC16004

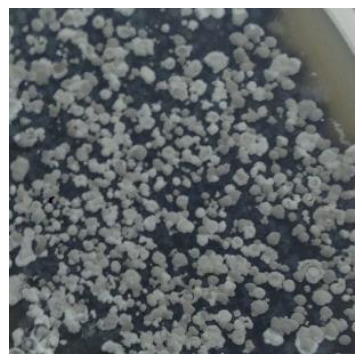

USC16008

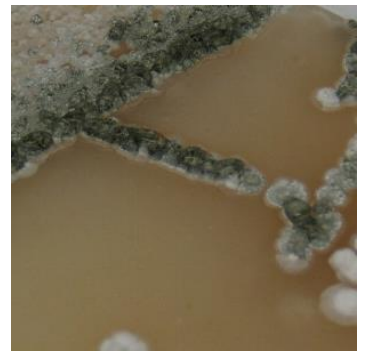

USC16012

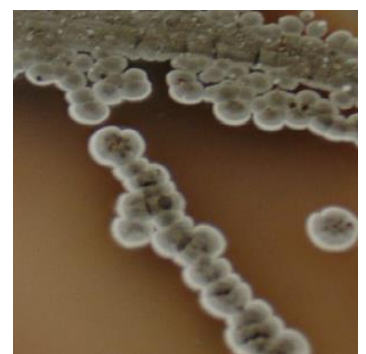

USC16017

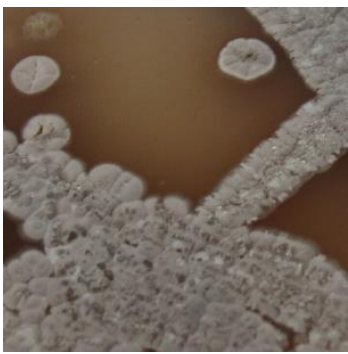

USC16001

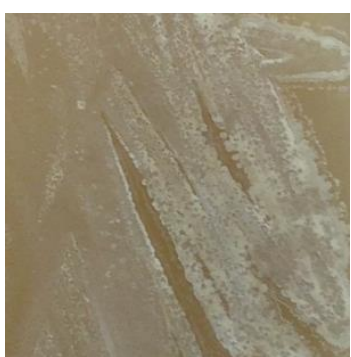

USC16005

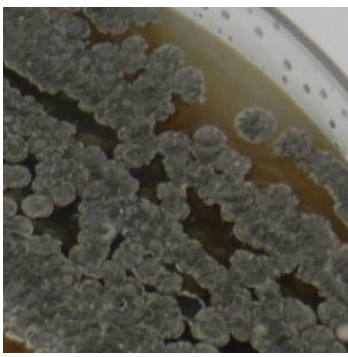

USC16009
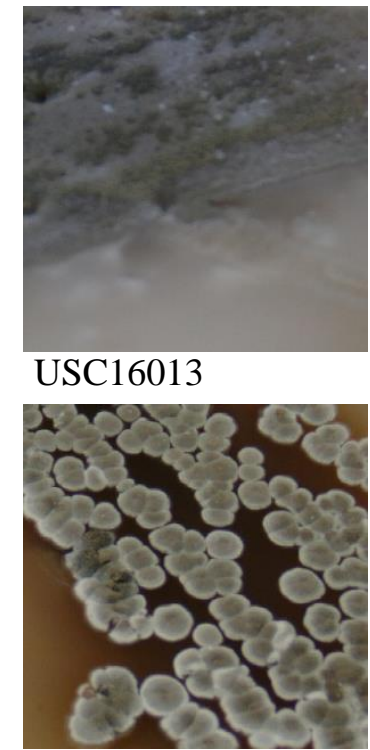

USC16018

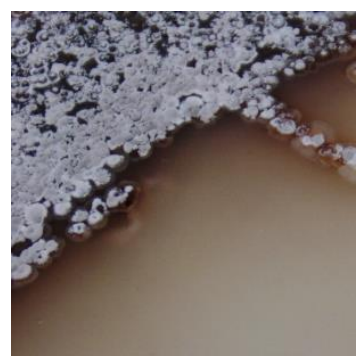

USC16002

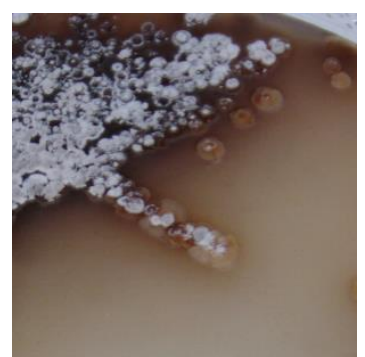

USC16006

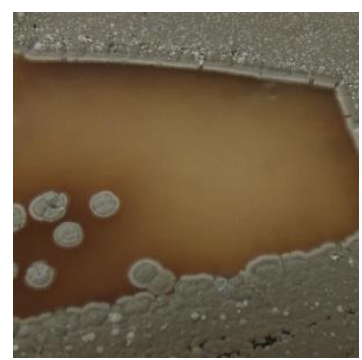

USC16010

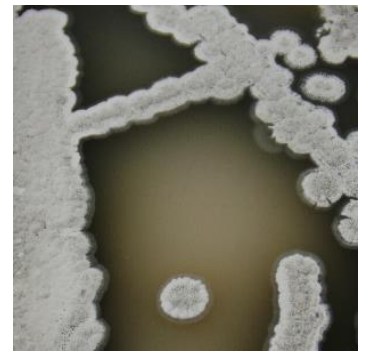

USC16014

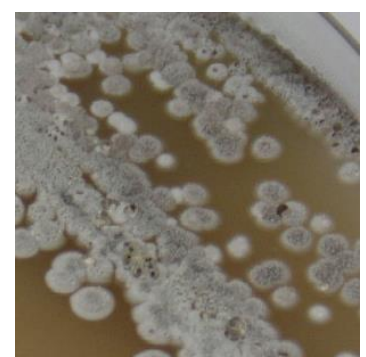

USC16019

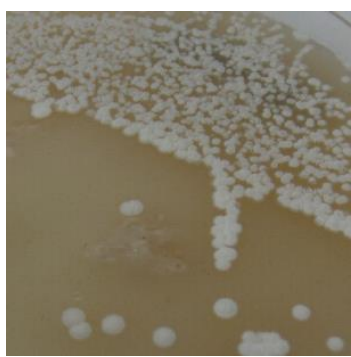

USC16003

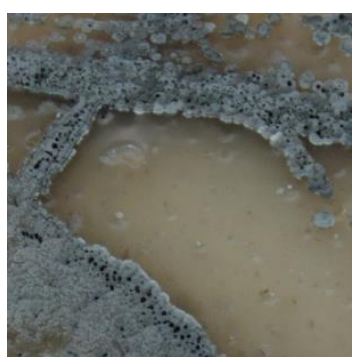

USC16007

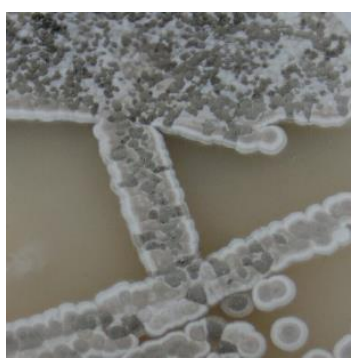

USC16011

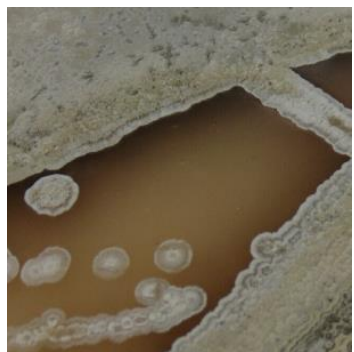

USC16015

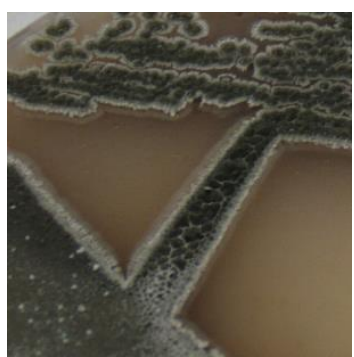

USC16020 
Appendix Ia: Ascidian-associated actinomycete library (USCUniversity of Sunshine Coast)

Streptomyces sp. isolates:
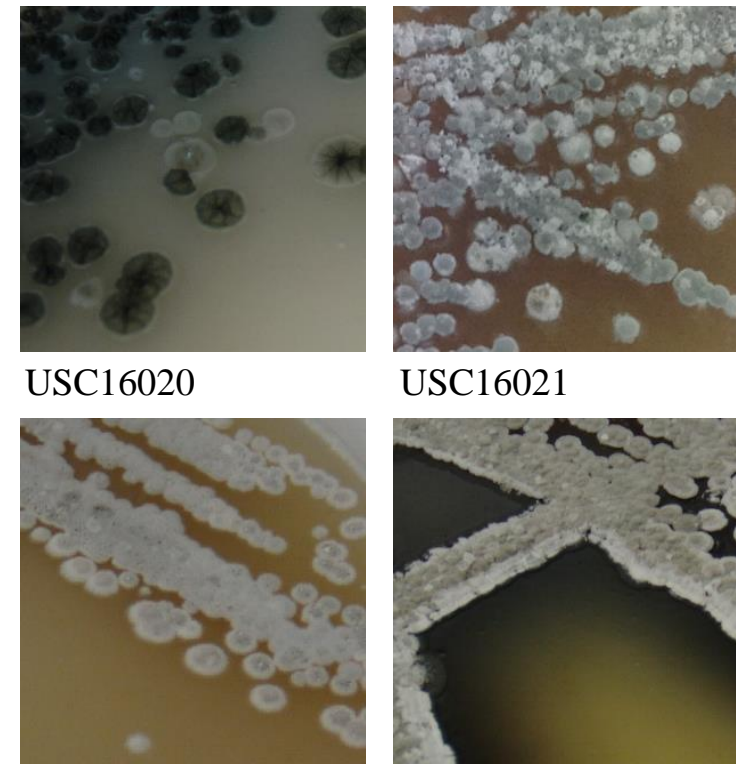

USC16024
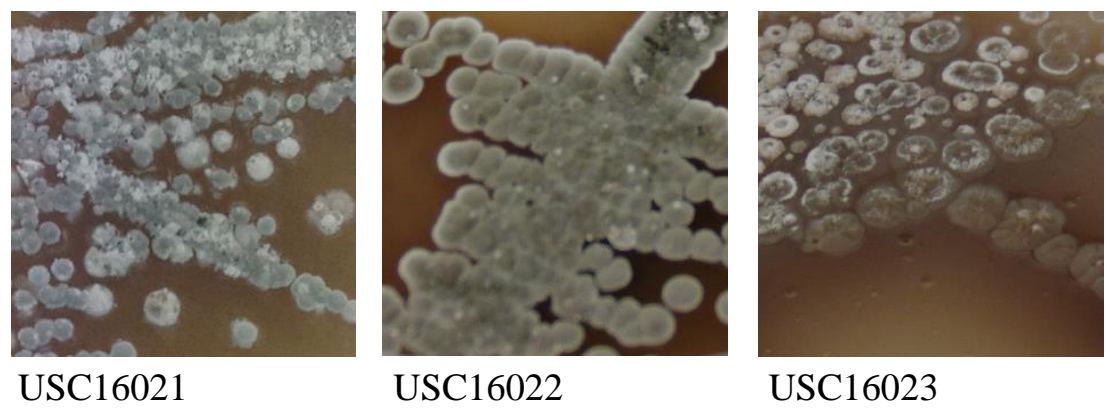

\section{USC16022}

USC16023

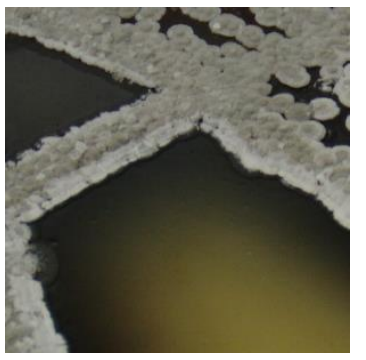

USC16025

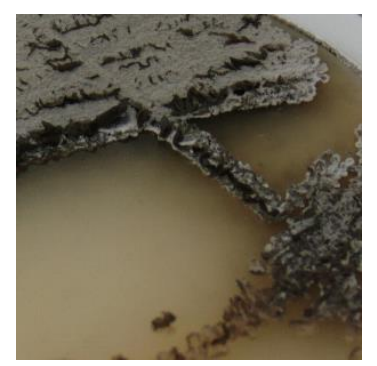

USC16026

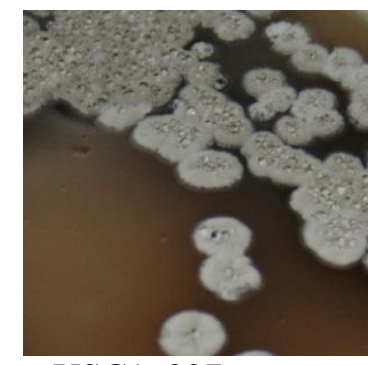

USC16027

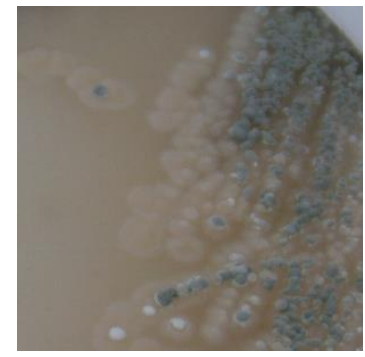

USC16062

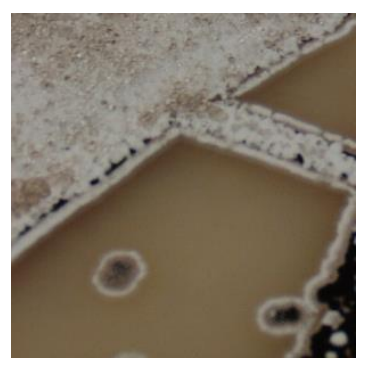

USC16098

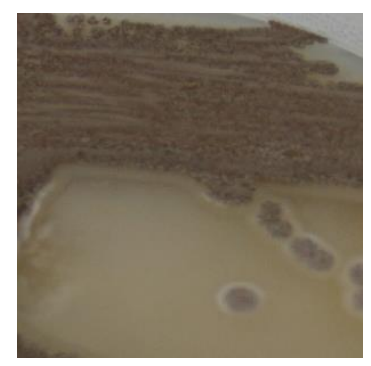

USC16099

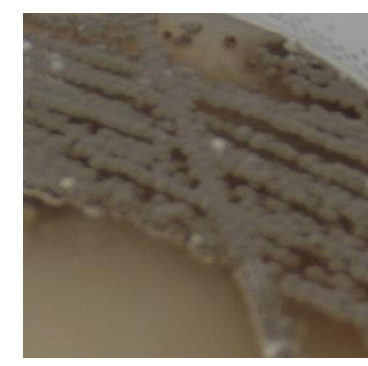

USC16100

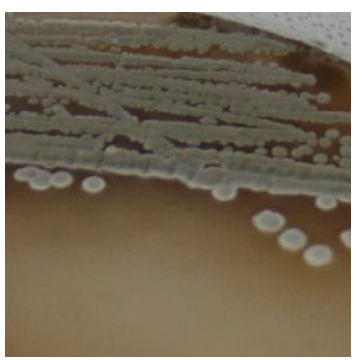

USC16101

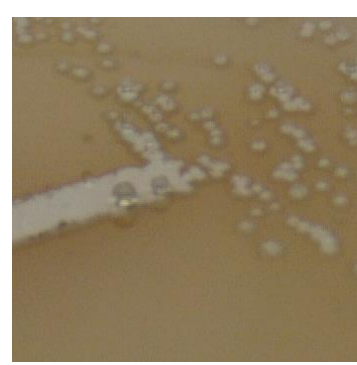

USC16113

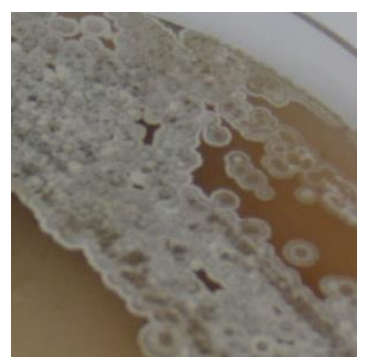

USC16115

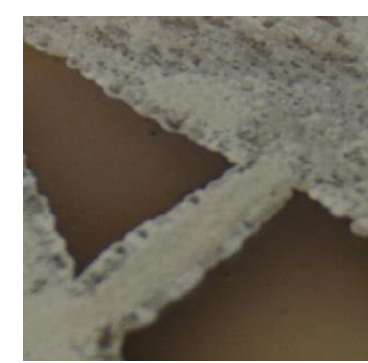

USC16117

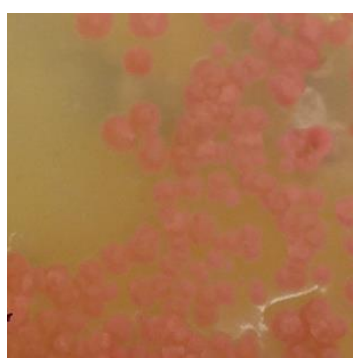

Streptosporangium sp. : USC16119

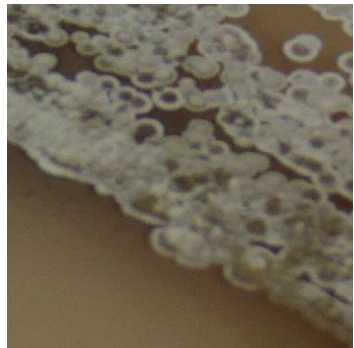

USC16118 
Appendix Ia: Ascidian-associated actinomycete library (USC- University of Sunshine Coast)

Micromonospora sp. isolates:

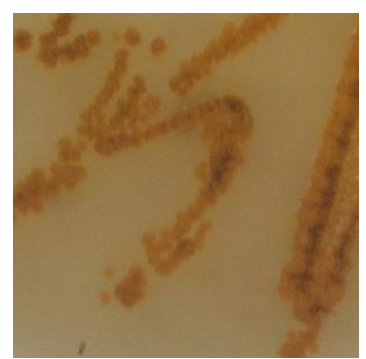

USC16028

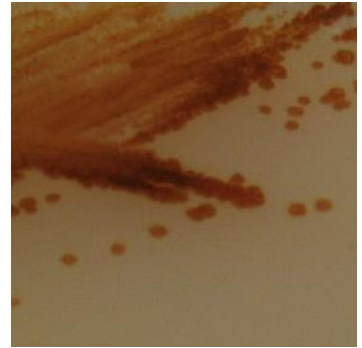

USC16032

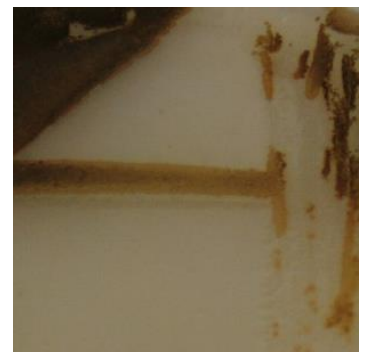

USC16036

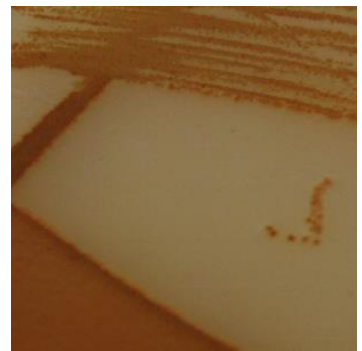

USC16040

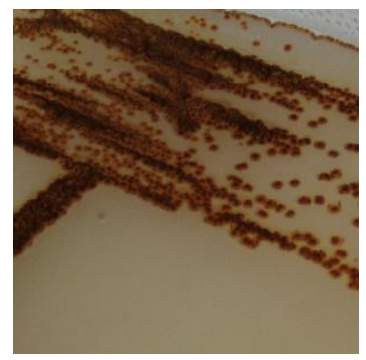

USC16045

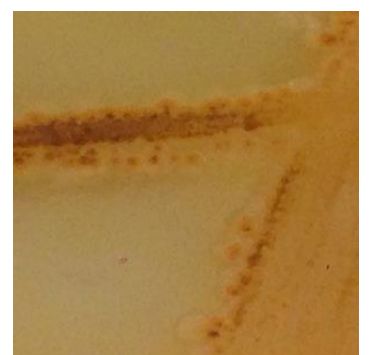

USC16029

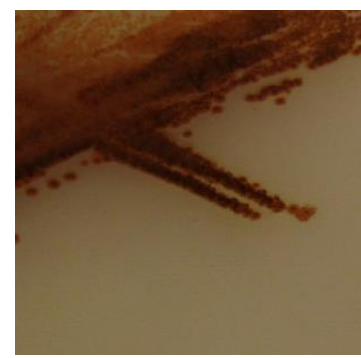

USC16033

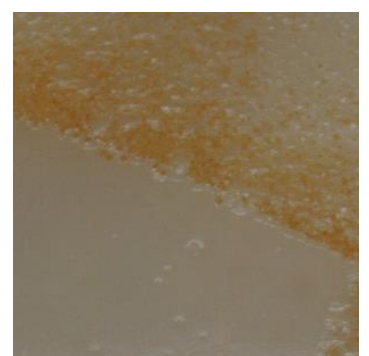

USC16037

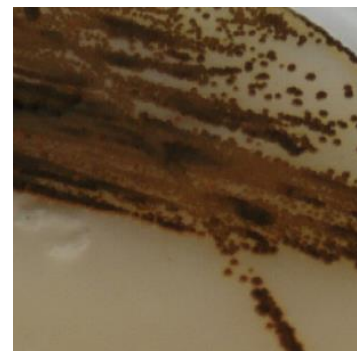

USC1642

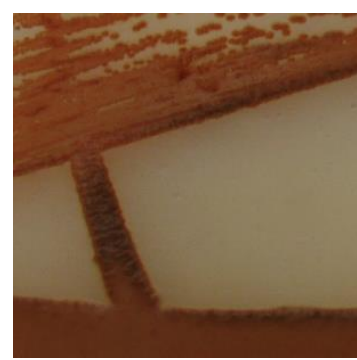

USC16046

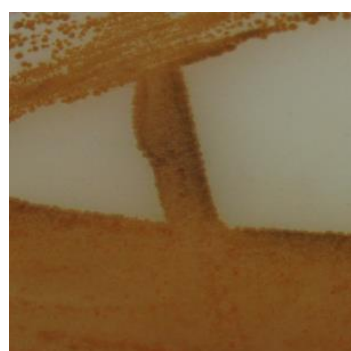

USC16030

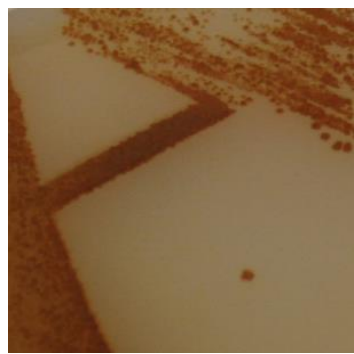

USC16034

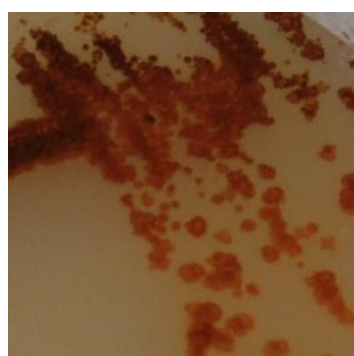

USC16038

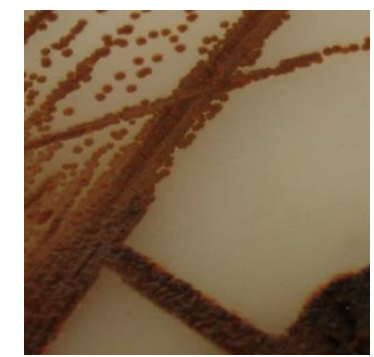

USC16043

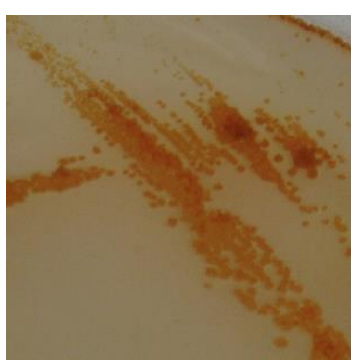

USC16047

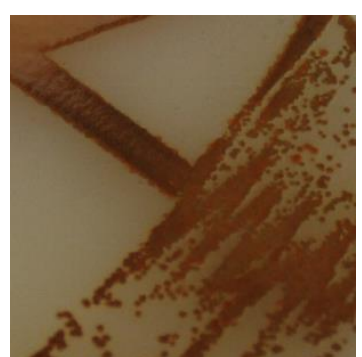

USC16031

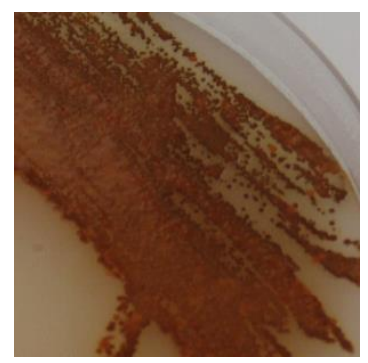

USC16035

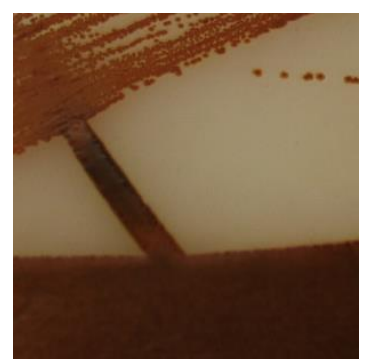

USC16039

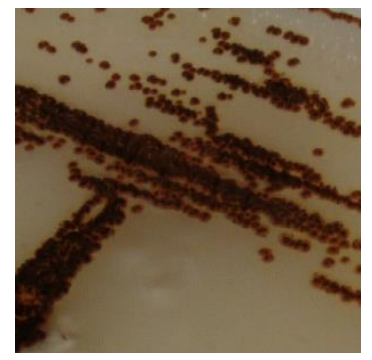

USC16044

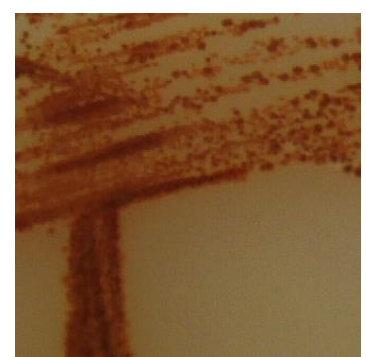

USC16048 
Appendix Ia: Ascidian-associated actinomycete library (USC- University of Sunshine Coast)

Micromonospora sp. isolates:

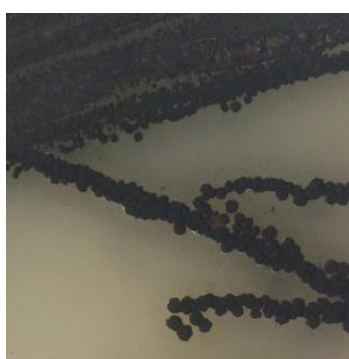

USC16049

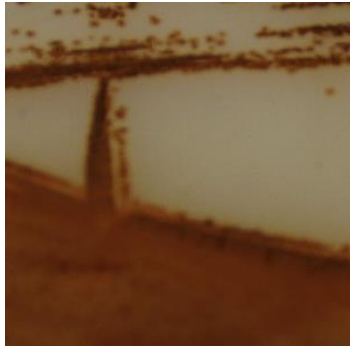

USC16053

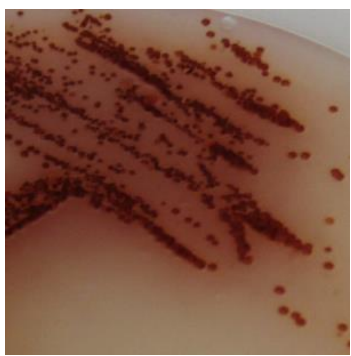

USC16057

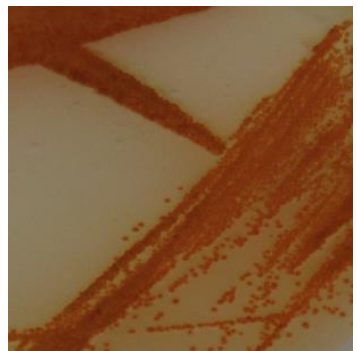

USC16061

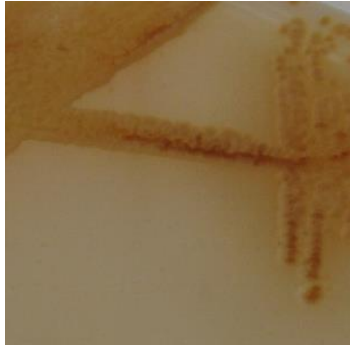

USC16067

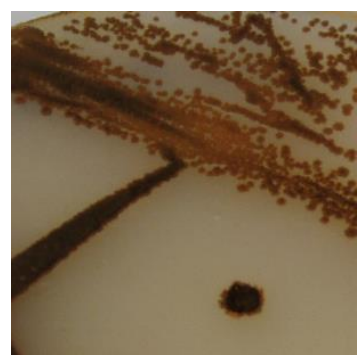

USC16050

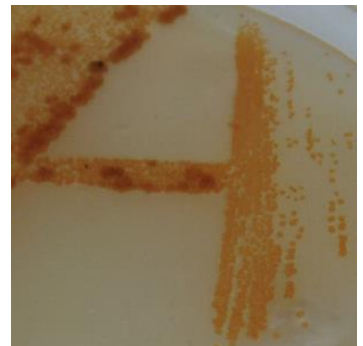

USC16054

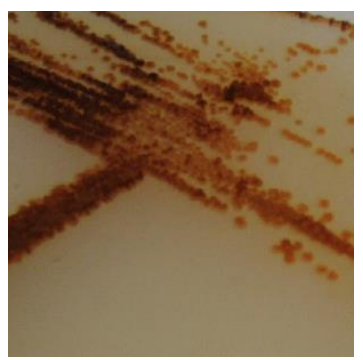

USC16058

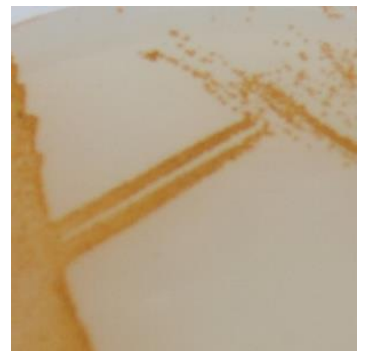

USC16064

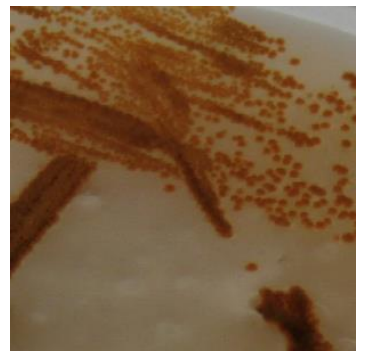

USC16068

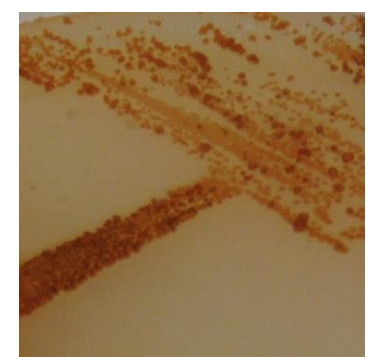

USC16051

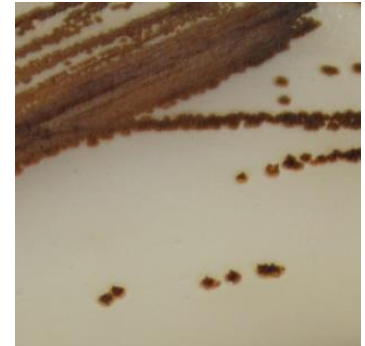

USC16055

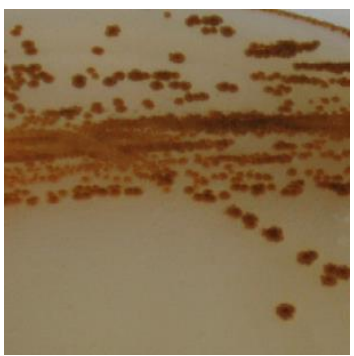

USC16059

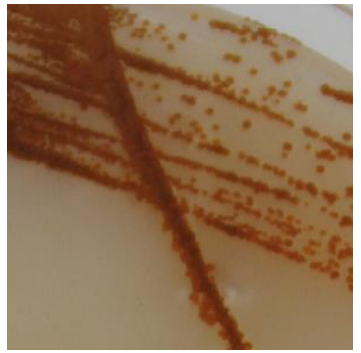

USC16065

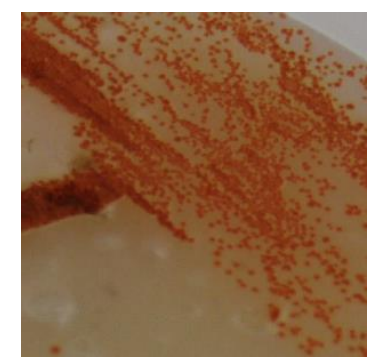

USC16069

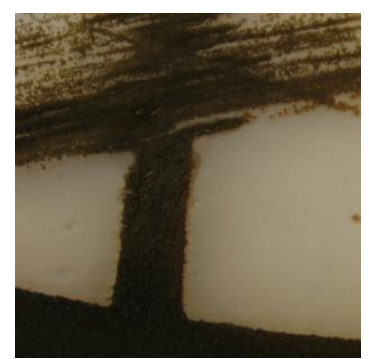

USC16052

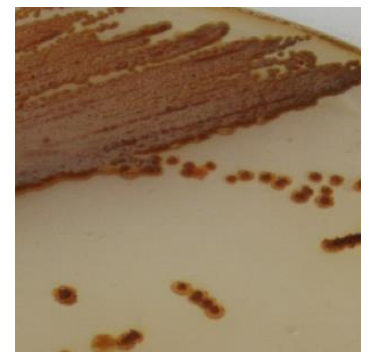

USC16056

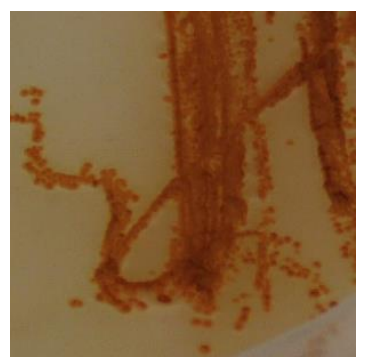

USC16060

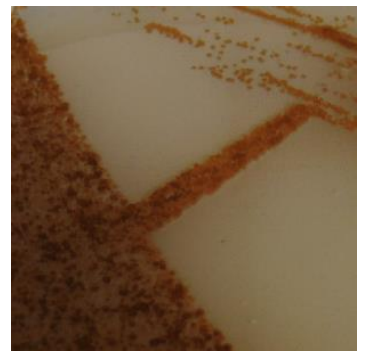

USC16066

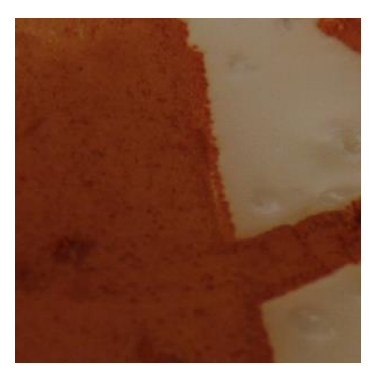

USC16070 
Appendix Ia: Ascidian-associated actinomycete library (USC- University of Sunshine Coast)

Micromonospora sp. isolates:

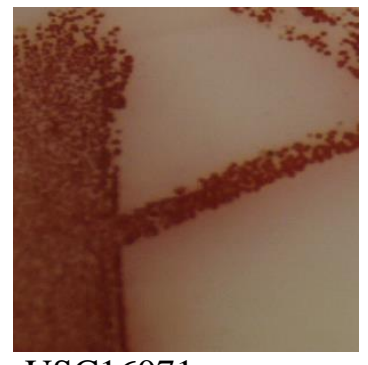

USC16071

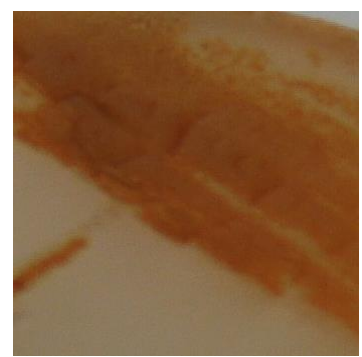

USC16076

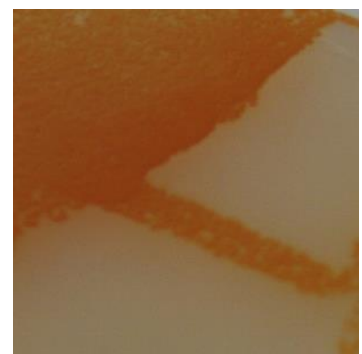

USC16080

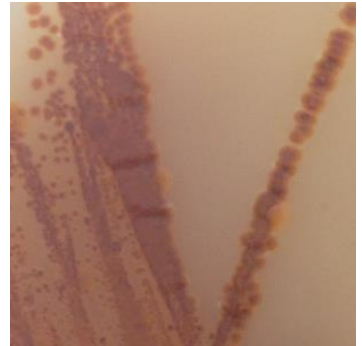

USC16084

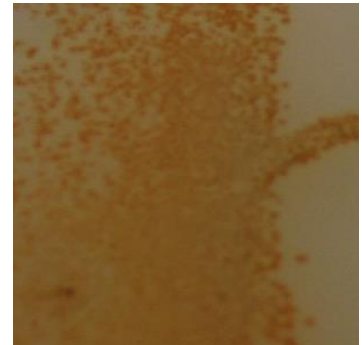

USC16088

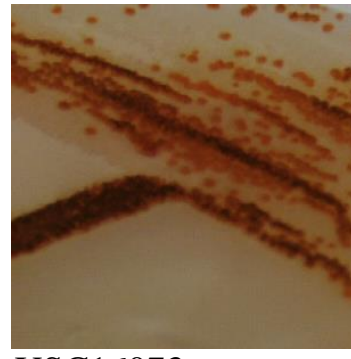

USC16073

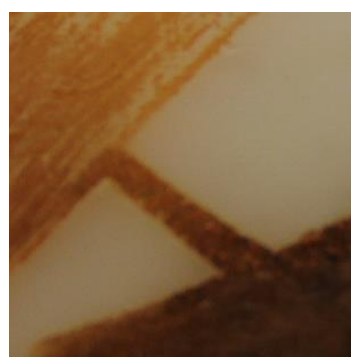

USC16077

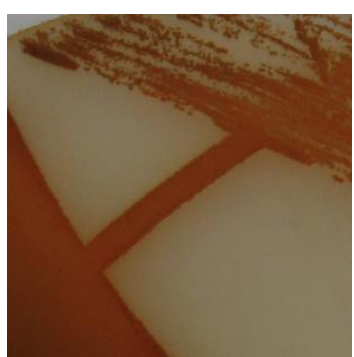

USC16081

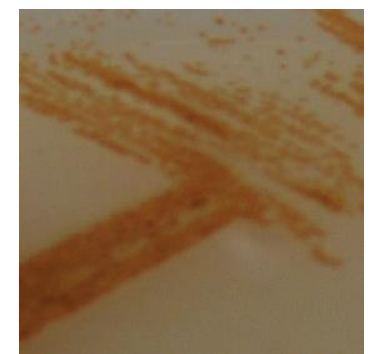

USC16085

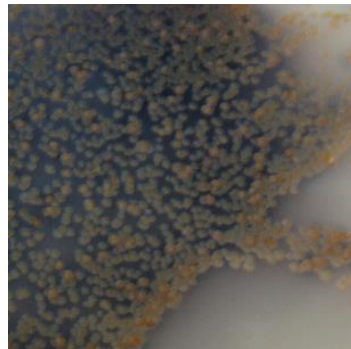

USC16089

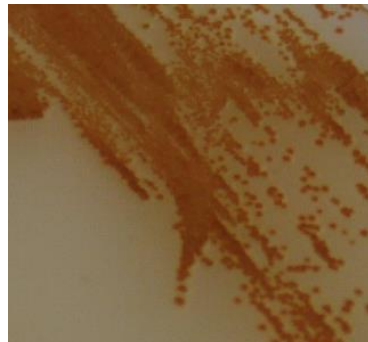

USC16074

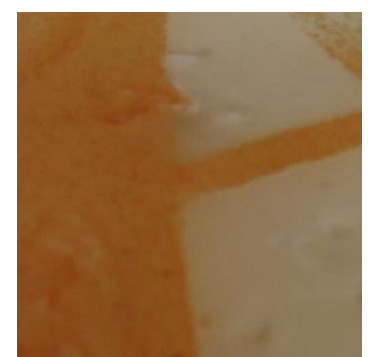

USC16078

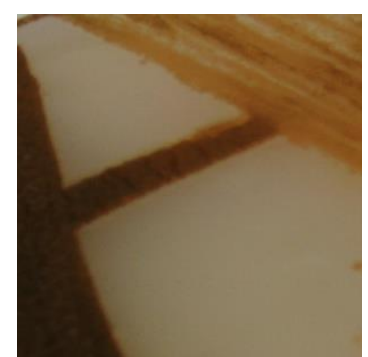

USC16082

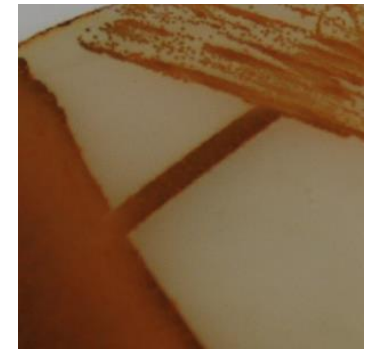

USC16086

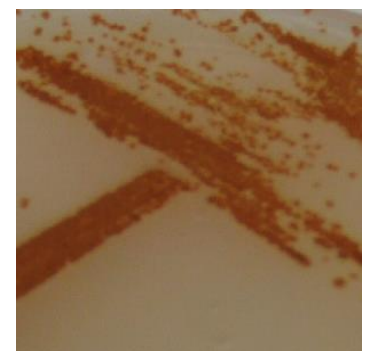

USC16090

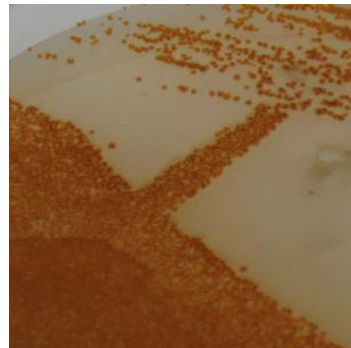

USC16075

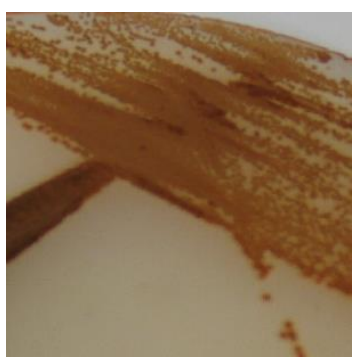

USC16079

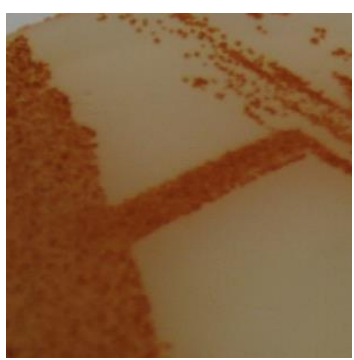

USC16083

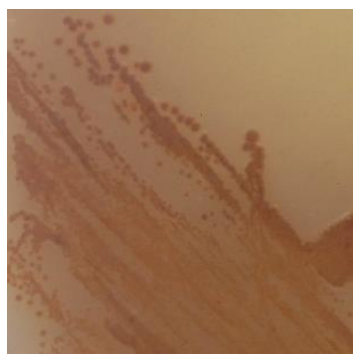

USC16087

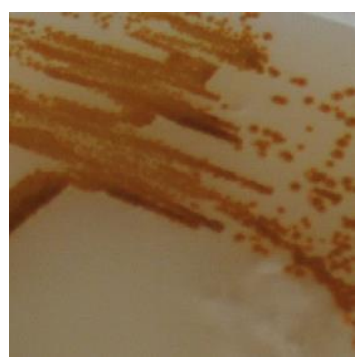

USC16102 
Appendix I: Ascidian-associated actinomycete library (USCUniversity of Sunshine Coast)

Micromonospora sp. and Nocardia form isolates:

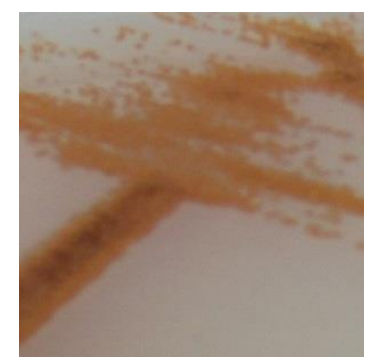

USC16103

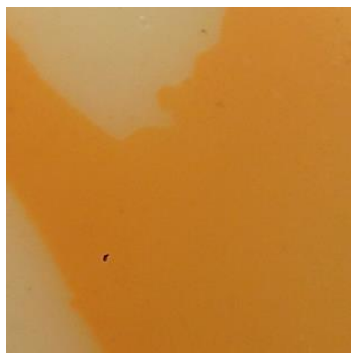

USC16107

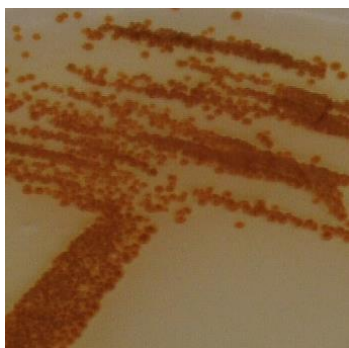

USC16112

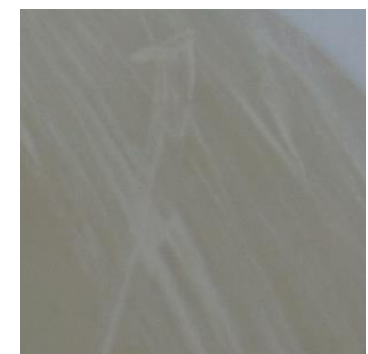

USC16091

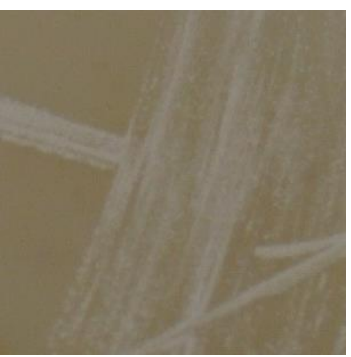

USC16095

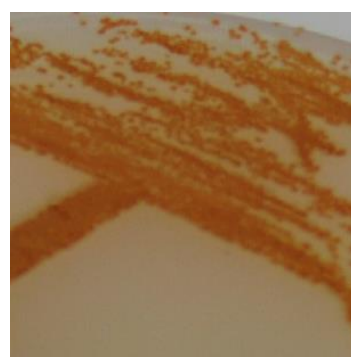

USC16104

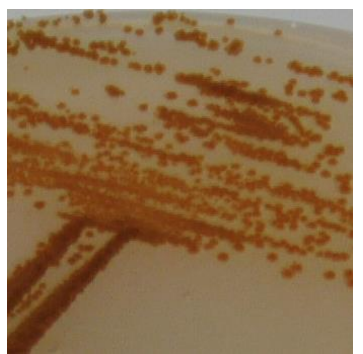

USC16108

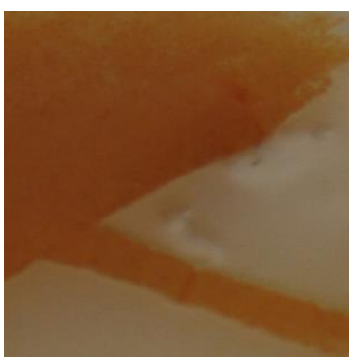

USC16105

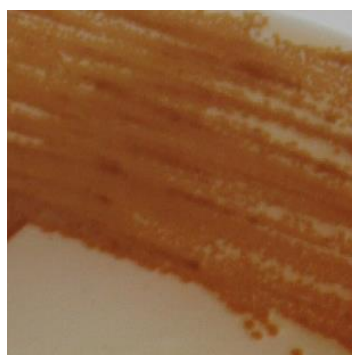

USC16109

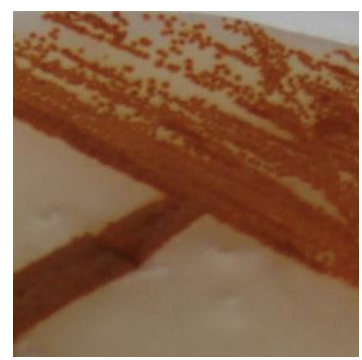

USC16106

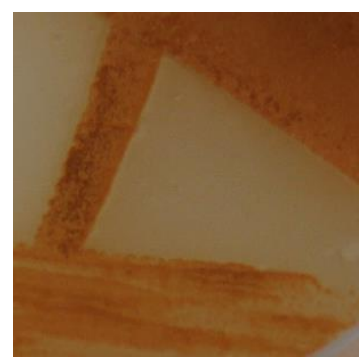

USC16111

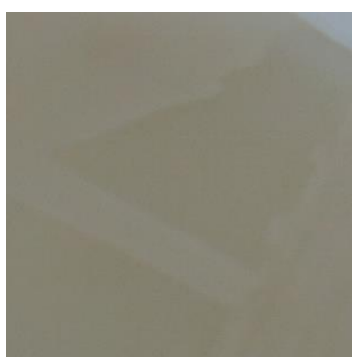

USC16092

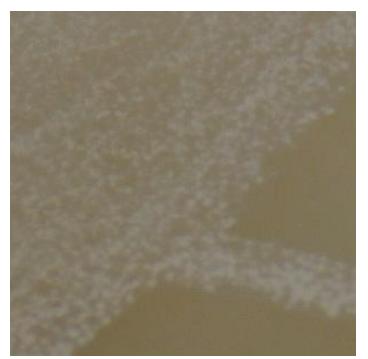

USC16096

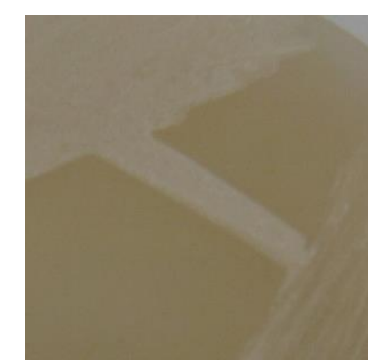

USC16093

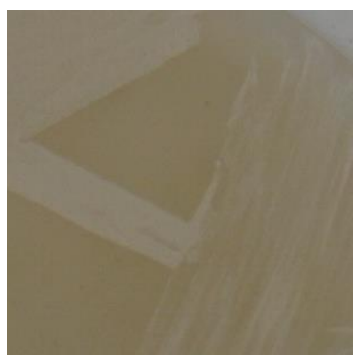

USC16097

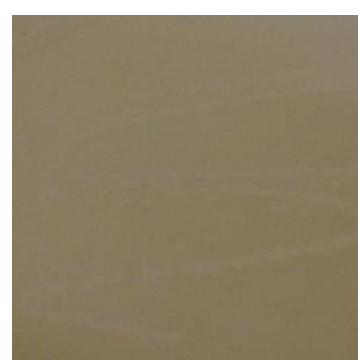

USC16094

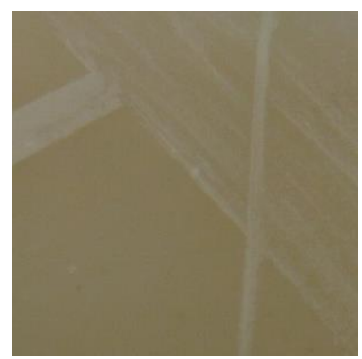

USC16120 


\section{Table IB. Molecular ion table - GNPS library hits}

\begin{tabular}{|c|c|c|c|c|c|c|c|c|c|c|c|c|c|c|}
\hline Parent Mass & $\mathbf{R T}(\min$ & rStder & L LibraryID & S. rubra. & A. solidum & P. vasculosum & Symplegma_associate & Aplidium_associate & Polyclinum_associate & Streptomyces & Micromonospora & Streptosporangium & Nocardia & Rhodococcus \\
\hline 495.899 & 11.83 & 0.54 & 1-Palmitoyl-sn-glycero-3-phosphocholine & 12 & 19 & 11 & 0 & 0 & 3 & 0 & 3 & 0 & 0 & 0 \\
\hline 479.9 & 11.48 & 0.60 & $4[5-[[4-[5-$-acetyl|(hydroxy)amino]pentylamino]-4-oxobutanoyl]-- & 25 & 5 & 15 & 9 & 2 & 4 & 0 & 14 & 0 & 1 & 0 \\
\hline 481.929 & 14.96 & 4.70 & LysoPAF & 10 & 5 & 1 & 0 & 0 & 0 & 0 & 0 & 0 & 0 & 0 \\
\hline 411.325 & 15.58 & 0.33 & NCGC00180497-02! & 4 & 4 & 0 & 0 & 0 & 5 & 0 & 5 & 0 & 0 & 0 \\
\hline 474.831 & 12.60 & 3.21 & Sildenafilititrate & 0 & 2 & 0 & 1 & 0 & 2 & 0 & 3 & 0 & 0 & 0 \\
\hline 127.055 & 1.92 & 0.01 & Thymine & 0 & 2 & 0 & 0 & 0 & 0 & 0 & 0 & 0 & 0 & 0 \\
\hline 137.054 & 1.41 & 0.02 & Hypoxanthine & 0 & 1 & 0 & 3 & 1 & 2 & 0 & 4 & 2 & 0 & 0 \\
\hline 105.076 & 7.46 & 2.50 & Benzaldehyde & 0 & 1 & 0 & 1 & 1 & 1 & 1 & 2 & 0 & 0 & 0 \\
\hline 482.369 & 11.12 & 4.59 & LysoPAF & 1 & 1 & 0 & 0 & 0 & 0 & 0 & 0 & 0 & 0 & 0 \\
\hline 261.14 & 2.44 & 0.35 & gamma-L-Glutamyl-L-leucine & 0 & 0 & 0 & 179 & 43 & 89 & 39 & 208 & 4 & 32 & 3 \\
\hline 261.029 & 3.54 & 0.10 & cyclo(Phe-4-Hyp) & 0 & 0 & 0 & 127 & 20 & 57 & 88 & 94 & 1 & 7 & 2 \\
\hline 211.077 & 3.32 & 1.02 & Cyclo(Pro-Leu) & 0 & 0 & 0 & 93 & 20 & 24 & 47 & 72 & 1 & 6 & 0 \\
\hline 247.063 & 1.84 & 0.10 & gamma-L-Glutamyl-L-valine & 0 & 0 & 0 & 64 & 16 & 33 & 22 & 60 & 3 & 14 & 2 \\
\hline 227.075 & 3.10 & 0.11 & cyclo(L-Leu-L-4-Hyp) & 0 & 0 & 0 & 63 & 18 & 35 & 50 & 52 & 0 & 5 & 0 \\
\hline 254.174 & 1.73 & 0.02 & Pheniramine formamide & 0 & 0 & 0 & 55 & 11 & 13 & 39 & 35 & 1 & 1 & 0 \\
\hline 426.648 & 3.96 & 0.35 & Leupeptin & 0 & 0 & 0 & 29 & 0 & 0 & 29 & 0 & 0 & 0 & 0 \\
\hline 1128.73 & 17.45 & 2.26 & 6,18,30-trimethyl-3,9,12,15,21,24,27,33,36-nona(propan-2-yl)-1,7,1: & 0 & 0 & 0 & 28 & 0 & 0 & 28 & 0 & 0 & 0 & 0 \\
\hline 245.026 & 4.53 & 0.03 & cyclo(L-Phe-D-Pro) & 0 & 0 & 0 & 23 & 1 & 8 & 8 & 20 & 0 & 0 & 0 \\
\hline 309.798 & 1.97 & 0.10 & Bestatin & 0 & 0 & 0 & 20 & 6 & 6 & 15 & 11 & 0 & 4 & 0 \\
\hline 426.277 & 9.15 & 0.13 & 1-tetradecanoyl-sn-glycero-3-phosphoethanolamine & 0 & 0 & 0 & 18 & 1 & 7 & 20 & 6 & 0 & 0 & 0 \\
\hline 585.34 & 2.29 & 0.03 & Desferrioxamine $\mathrm{B}+\mathrm{Al}$ & 0 & 0 & 0 & 18 & 4 & 3 & 0 & 23 & 2 & 0 & 0 \\
\hline 912.889 & 7.61 & 0.08 & Surugamide_A & 0 & 0 & 0 & 15 & 0 & 3 & 18 & 0 & 0 & 0 & 0 \\
\hline 182.091 & 1.52 & 0.03 & Tyrosine & 0 & 0 & 0 & 15 & 0 & 1 & 9 & 6 & 0 & 1 & 0 \\
\hline 188.08 & 2.76 & 0.06 & Metrazoline & 0 & 0 & 0 & 14 & 0 & 3 & 8 & 6 & 0 & 3 & 0 \\
\hline 227.15 & 3.14 & 0.12 & cyclo(L-Leu-L-4-Hyp) & 0 & 0 & 0 & 13 & 2 & 6 & 8 & 11 & 1 & 0 & 0 \\
\hline 132.11 & 1.45 & 0.62 & Leucine & 0 & 0 & 1 & 13 & 3 & 4 & 11 & 6 & 0 & 1 & 1 \\
\hline 197.138 & 3.10 & 0.01 & cyclo(L-Val-L-Pro) & 0 & 0 & 0 & 13 & 0 & 1 & 4 & 9 & 0 & 1 & 0 \\
\hline 260.828 & 2.46 & 0.25 & gamma-L-Glutamyl-L-leucine & 0 & 0 & 0 & 12 & 6 & 7 & 1 & 16 & 0 & 4 & 0 \\
\hline 293.046 & 1.13 & 0.02 & Vanillylnonanamide & 0 & 0 & 0 & 10 & 0 & 1 & 9 & 1 & 0 & 1 & 0 \\
\hline 220.13 & 2.16 & 0.06 & Vitamin B5 & 0 & 0 & 0 & 10 & 0 & 0 & 10 & 0 & 0 & 0 & 0 \\
\hline 143.091 & 1.04 & 0.01 & Ectoine & 0 & 0 & 0 & 8 & 2 & 1 & 5 & 1 & 0 & 4 & 0 \\
\hline 169.084 & 3.59 & 0.03 & Norharmane & 0 & 0 & 0 & 6 & 1 & 2 & 3 & 4 & 0 & 0 & 0 \\
\hline 403.758 & 14.39 & 0.44 & 2-(14-methylpentadecanoylamino)-3-phenylpropanoic acid & 0 & 0 & 0 & 6 & 0 & 1 & 7 & 0 & 0 & 0 & 0 \\
\hline 621.434 & 12.89 & 0.02 & Rakicidin B & 0 & 0 & 0 & 6 & 1 & 0 & 0 & 5 & 2 & 0 & 0 \\
\hline 487.18 & 7.02 & 0.01 & (3R)-4-[6-[(2R,4R,5S,6R)-4,5-dihydroxy-6-methyloxan-2-yl]-1,5-dit & 0 & 0 & 0 & 6 & 0 & 0 & 6 & 0 & 0 & 0 & 0 \\
\hline 118.094 & 1.32 & 0.25 & Betaine & 0 & 0 & 0 & 6 & 0 & 0 & 4 & 1 & 0 & 1 & 0 \\
\hline 310.865 & 2.23 & 0.01 & gamma-Glutamyltyrosine & 0 & 0 & 0 & 6 & 0 & 0 & 0 & 6 & 0 & 0 & 0 \\
\hline 124.047 & 1.38 & 0.01 & Vitamin B3 & 0 & 0 & 0 & 5 & 0 & 3 & 2 & 5 & 0 & 0 & 0 \\
\hline 150.068 & 1.63 & 0.31 & Methionine & 0 & 0 & 0 & 5 & 2 & 0 & 0 & 0 & 0 & 5 & 0 \\
\hline 260.887 & 3.57 & 0.13 & cyclo(Phe-4-Hyp) & 0 & 0 & 0 & 5 & 0 & 0 & 2 & 3 & 0 & 0 & 0 \\
\hline 181.607 & 1.52 & 0.03 & L-Tyrosine & 1 & 0 & 1 & 4 & 0 & 1 & 2 & 3 & 0 & 0 & 0 \\
\hline 166.014 & 1.93 & 0.14 & Phenylalanine & 0 & 0 & 0 & 4 & 0 & 6 & 5 & 5 & 0 & 0 & 0 \\
\hline 426.074 & 9.25 & 0.09 & 1-tetradecanoyl-sn-glycero-3-phosphoethanolamine & 0 & 0 & 0 & 4 & 0 & 2 & 4 & 2 & 0 & 0 & 0 \\
\hline 597.22 & 10.24 & 0.01 & (3R)-4[6-[(2S,4aS,5aR,9R,9aR,10aR)-2,9-dimethyl-3-oxo-4,4a, 5a,6, & 0 & 0 & 0 & 4 & 0 & 0 & 4 & 0 & 0 & 0 & 0 \\
\hline 298.112 & 2.53 & 0.02 & 5-Methylthioadenosine & 0 & 0 & 0 & 4 & 0 & 0 & 2 & 2 & 0 & 0 & 0 \\
\hline
\end{tabular}




\section{Table IB. Molecular ion table - GNPS library hits (continued)}

\begin{tabular}{|c|c|c|c|c|c|c|c|c|c|c|c|c|c|c|}
\hline parent mass & $\mathbf{R T}(\min$ & r Stdder & rLibraryII & S. rubra & A. solidum & P. vasculosum & Symplegma_associate & Aplidium_associate & Polyclinum_associate & Streptomyces & Micromonospora & Streptosporangium & Nocardia & Rhodococcus \\
\hline 429.333 & 3.94 & 0.09 & Leupeptin hemisulfate salt & 0 & 0 & 0 & 4 & 0 & 0 & 4 & 0 & 0 & 0 & 0 \\
\hline 261.136 & 3.50 & 0.03 & cyclo(Phe-4-Hyp) & 0 & 0 & 0 & 3 & 0 & 1 & 2 & 2 & 0 & 0 & 0 \\
\hline 146.101 & 1.06 & 0.01 & Glutamic acid & 0 & 0 & 0 & 3 & 3 & 0 & 0 & 6 & 0 & 0 & 0 \\
\hline 296.325 & 4.68 & 0.05 & methyl 2-[(3-formamido-2-hydroxybenzoyl)amino]-3-hydroxybute & 0 & 0 & 0 & 3 & 0 & 0 & 3 & 0 & 0 & 0 & 0 \\
\hline 296.29 & 4.65 & 0.03 & methyl 2-[(3-formamido-2-hydroxybenzoyl)amino]-3-hydroxybuta & 0 & 0 & 0 & 3 & 0 & 0 & 3 & 0 & 0 & 0 & 0 \\
\hline 267.614 & 1.72 & 0.20 & Adenosine & 2 & 0 & 0 & 2 & 0 & 0 & 1 & 1 & 0 & 0 & 0 \\
\hline 426.387 & 15.20 & 0.18 & Ethmozine & 0 & 0 & 0 & 2 & 1 & 2 & 3 & 1 & 0 & 0 & 0 \\
\hline 153.786 & 16.47 & 1.50 & Acetamide & 0 & 0 & 0 & 2 & 0 & 2 & 2 & 2 & 0 & 0 & 0 \\
\hline 132.11 & 1.21 & 0.18 & L-Leucine & 0 & 0 & 0 & 2 & 2 & 1 & 3 & 2 & 0 & 0 & 0 \\
\hline 267.261 & 1.61 & 0.04 & Adenosine & 0 & 0 & 0 & 2 & 1 & 1 & 1 & 1 & 1 & 0 & 0 \\
\hline 227.147 & 3.15 & 0.10 & cyclo-[L-(4-hydroxy-Pro)-L-leu] & 0 & 0 & 0 & 2 & 0 & 1 & 1 & 2 & 0 & 0 & 0 \\
\hline 526.969 & 1.10 & 0.09 & Polysaccharide Hexose $x 3$ & 0 & 0 & 0 & 2 & 0 & 1 & 2 & 1 & 0 & 0 & 0 \\
\hline 364.857 & 1.06 & 0.09 & sucrose & 0 & 0 & 0 & 2 & 0 & 1 & 1 & 2 & 0 & 0 & 0 \\
\hline 178.095 & 2.19 & 0.01 & 3-Amino-3-(4-hydroxyphenyl)propionate & 0 & 0 & 0 & 2 & 0 & 0 & 2 & 0 & 0 & 0 & 0 \\
\hline 257.222 & 6.42 & 0.01 & 5-beta-Androstane-3-alpha & 0 & 0 & 0 & 2 & 0 & 0 & 2 & 0 & 0 & 0 & 0 \\
\hline 211.118 & 2.03 & 0.02 & cyclo(L-Leu-L-Pro) & 0 & 0 & 0 & 2 & 0 & 0 & 1 & 1 & 0 & 0 & 0 \\
\hline 205.119 & 1.94 & 1.22 & Tryptophan & 0 & 0 & 0 & 2 & 0 & 0 & 1 & 0 & 0 & 1 & 0 \\
\hline 205.107 & 2.75 & 0.08 & Tryptophan & 0 & 0 & 0 & 2 & 0 & 0 & 2 & 0 & 0 & 0 & 0 \\
\hline 152.066 & 1.39 & 0.28 & Xanthine & 3 & 0 & 0 & 1 & 0 & 0 & 0 & 1 & 0 & 0 & 0 \\
\hline 144.089 & 3.23 & 0.22 & Quinolin-4-ol & 0 & 0 & 0 & 1 & 2 & 2 & 1 & 4 & 0 & 0 & 0 \\
\hline 227.149 & 3.25 & 0.02 & cyclo(L-Leu-L-4hydroxy-Pro) & 0 & 0 & 0 & 1 & 0 & 1 & 1 & 1 & 0 & 0 & 0 \\
\hline 247.141 & 1.88 & 0.07 & gamma-L-Glutamyl-L-valine & 0 & 0 & 0 & 1 & 0 & 0 & 0 & 1 & 0 & 0 & 0 \\
\hline 194.572 & 2.15 & 0.53 & Caffeine & 12 & 0 & 0 & 0 & 0 & 0 & 0 & 0 & 0 & 0 & 0 \\
\hline 429.023 & 4.81 & 0.03 & 8-desoxyenterocin & 0 & 0 & 0 & 0 & 0 & 12 & 12 & 0 & 0 & 0 & 0 \\
\hline 521.431 & 11.18 & 0.55 & 1-Oleoyl-sn-glycero-3-phosphocholine & 0 & 0 & 2 & 0 & 0 & 4 & 0 & 4 & 0 & 0 & 0 \\
\hline 245.139 & 4.55 & 0.02 & cyclo(L-Phe-D-Pro) & 0 & 0 & 0 & 0 & 1 & 1 & 1 & 1 & 0 & 0 & 0 \\
\hline 592.93 & 14.85 & 0.02 & Pheophorbide A & 0 & 0 & 3 & 0 & 0 & 0 & 0 & 0 & 0 & 0 & 0 \\
\hline 154.165 & 16.51 & 2.59 & Xanthine & 0 & 0 & 1 & 0 & 1 & 0 & 1 & 0 & 0 & 0 & 0 \\
\hline 154.167 & 18.16 & 0.04 & L-Histidine & 0 & 0 & 0 & 0 & 1 & 0 & 1 & 0 & 0 & 0 & 0 \\
\hline
\end{tabular}




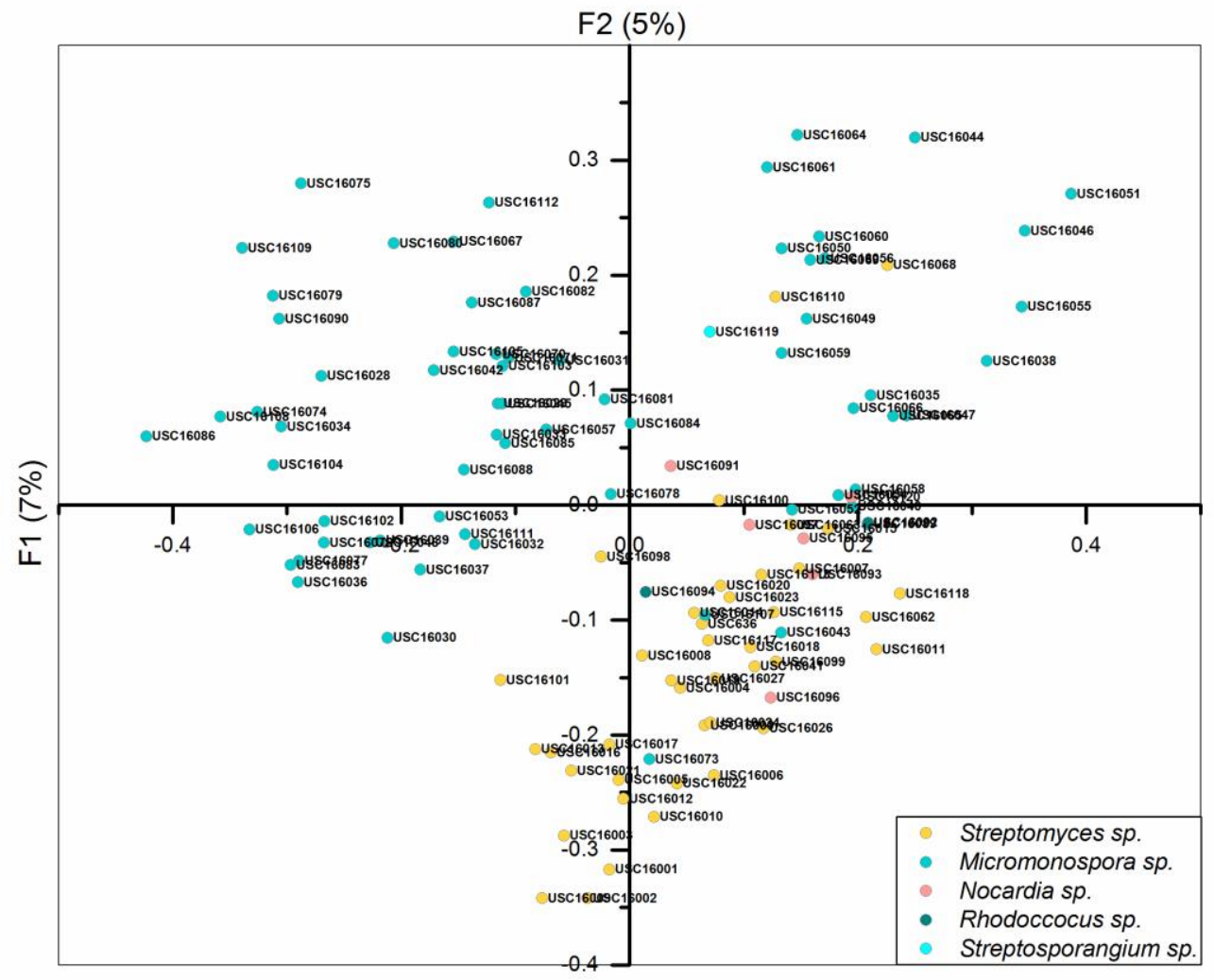

Figure IIBa. PCoA plot of HSQC-TOCSY all PoI data of 119 actinomycete stains labelled with their isolate number

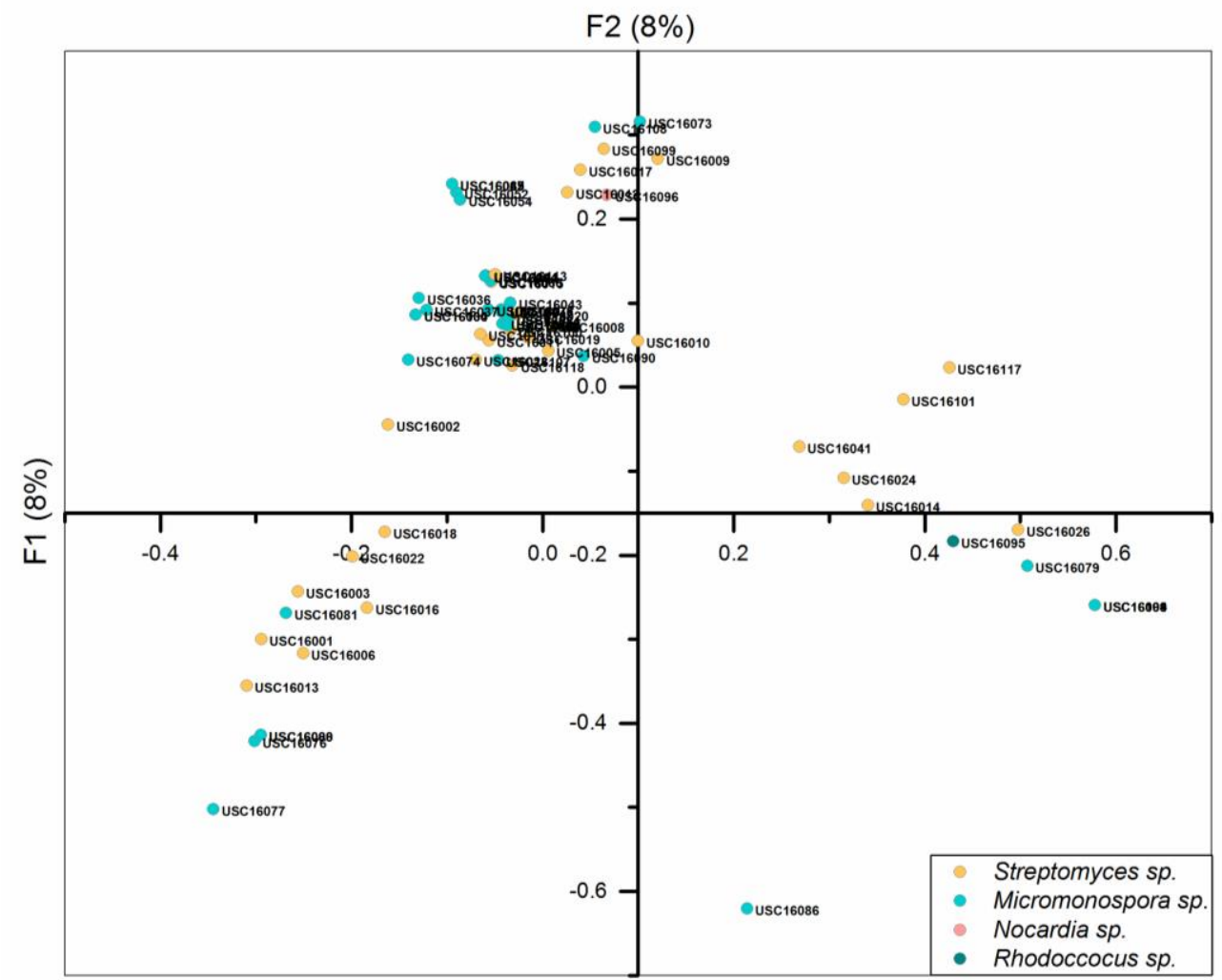

Figure IIBb. PCoA plot of HSQC-TOCSY PK PoI data of 119 actinomycete stains labelled with their isolate number 


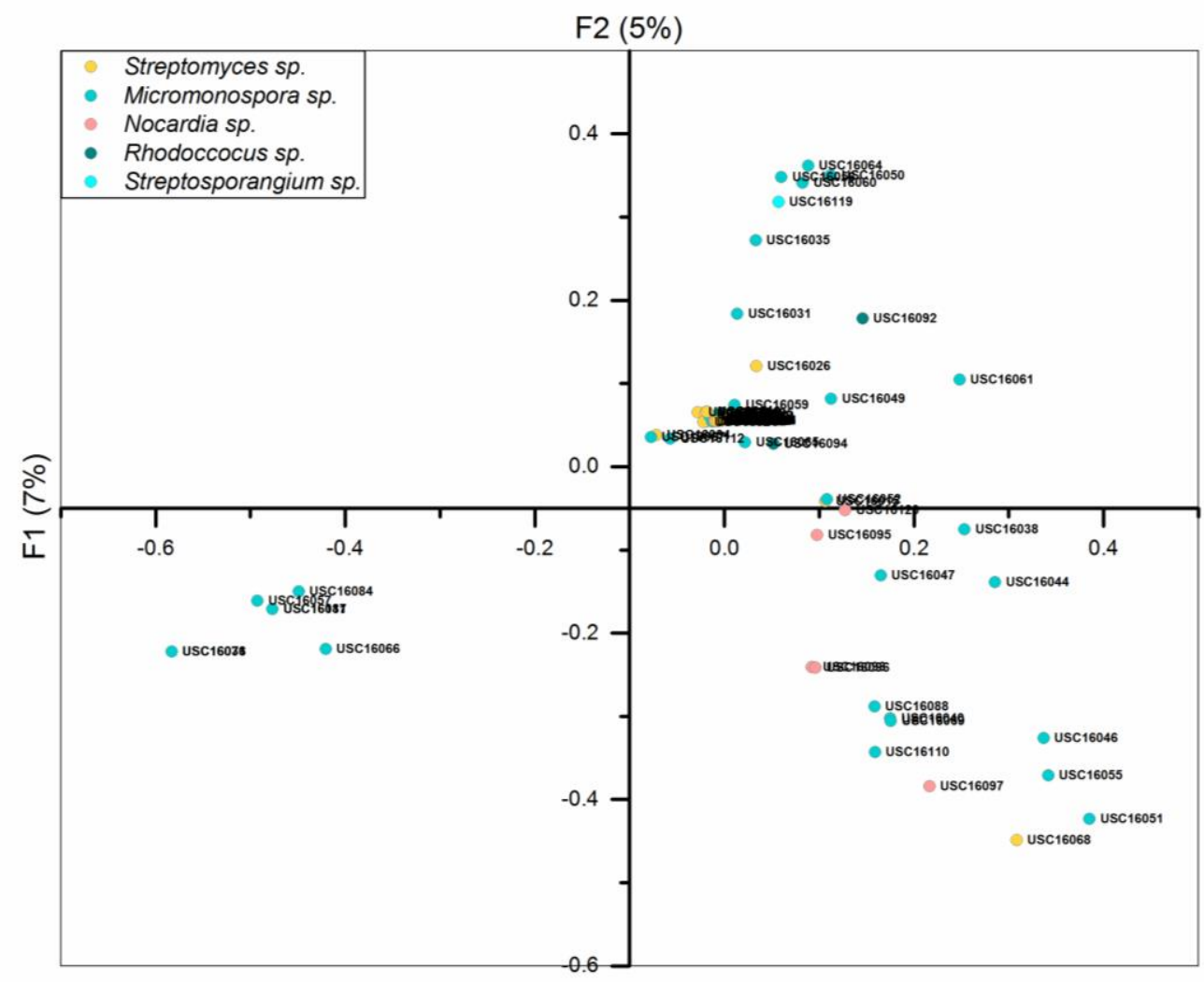

Figure IIBc. PCoA plot of HSQC-TOCSY PEP PoI data of 119 actinomycete stains labelled with their isolate number 


\section{Appendix II-for Chapter 3}

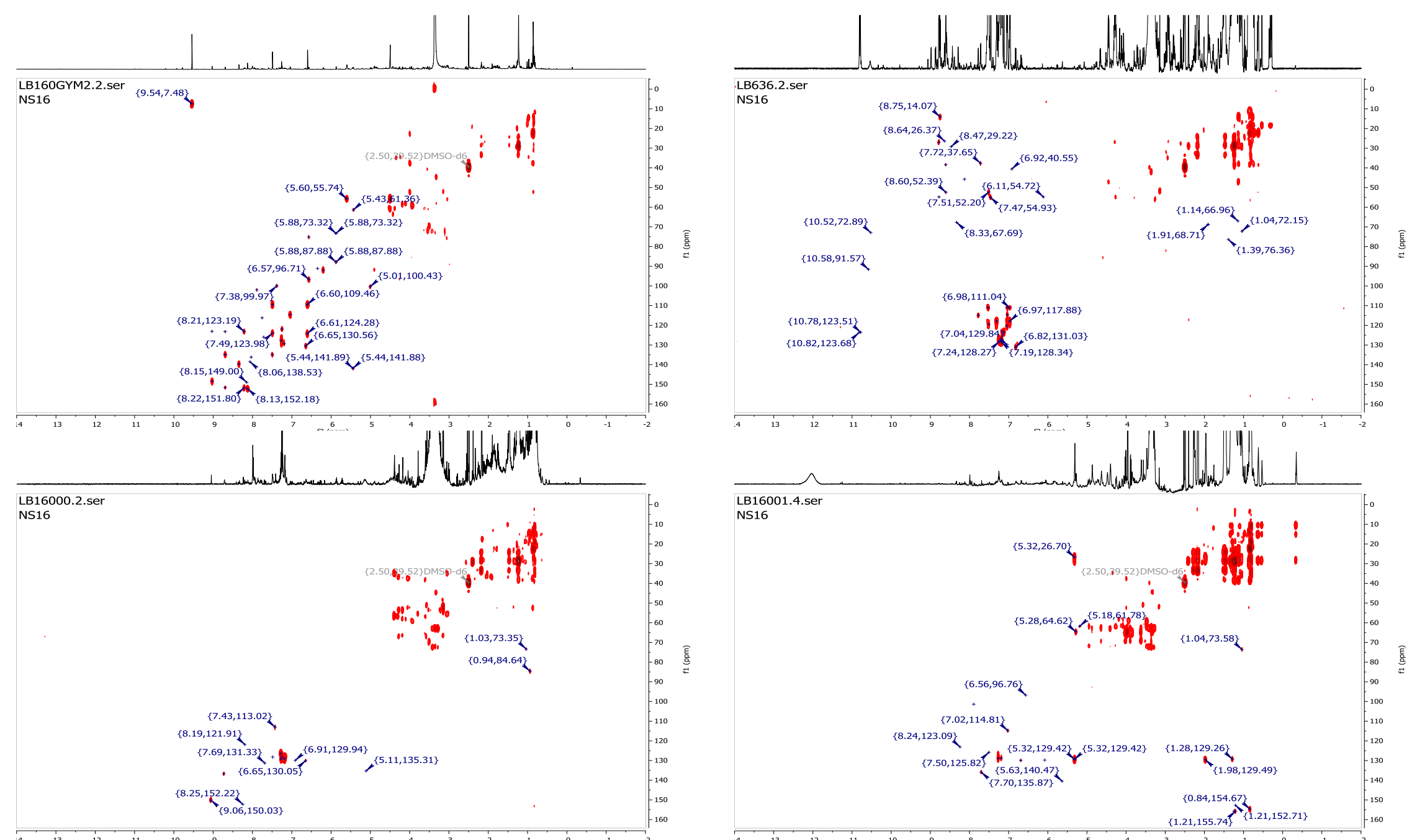

Figure IIA. HSQC-TOCSY NMR spectra of actinomycete crude extracts (DMSO- $d_{6}$; $800 \mathrm{MHz}$; USC-isolate number given in the top left corner of spectra) 

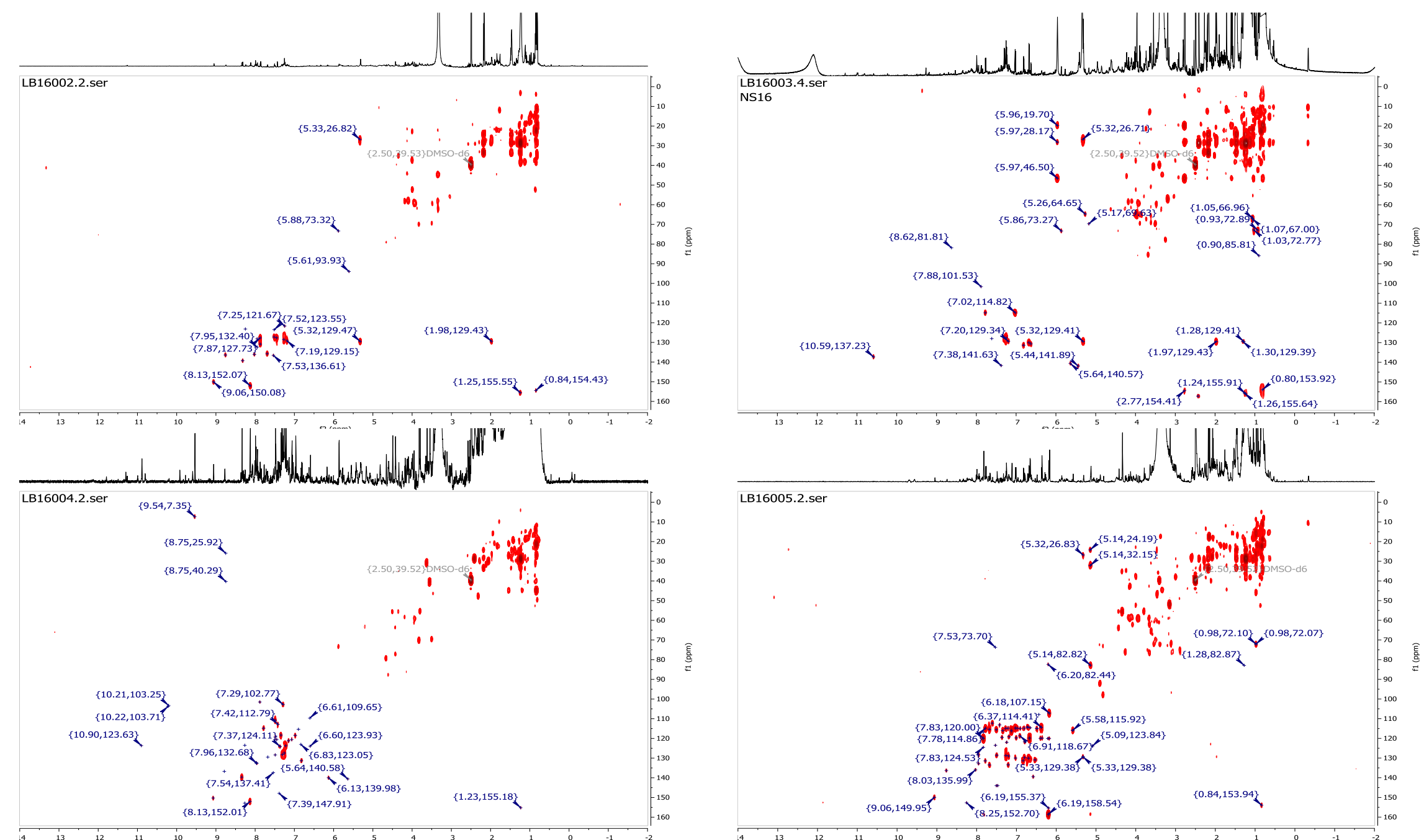

Figure IIA. HSQC-TOCSY NMR spectra of actinomycete crude extracts (continued) (DMSO- $d_{6 ;} 800 \mathrm{MHz}$; USC-isolate number given in the top left corner of spectra) 

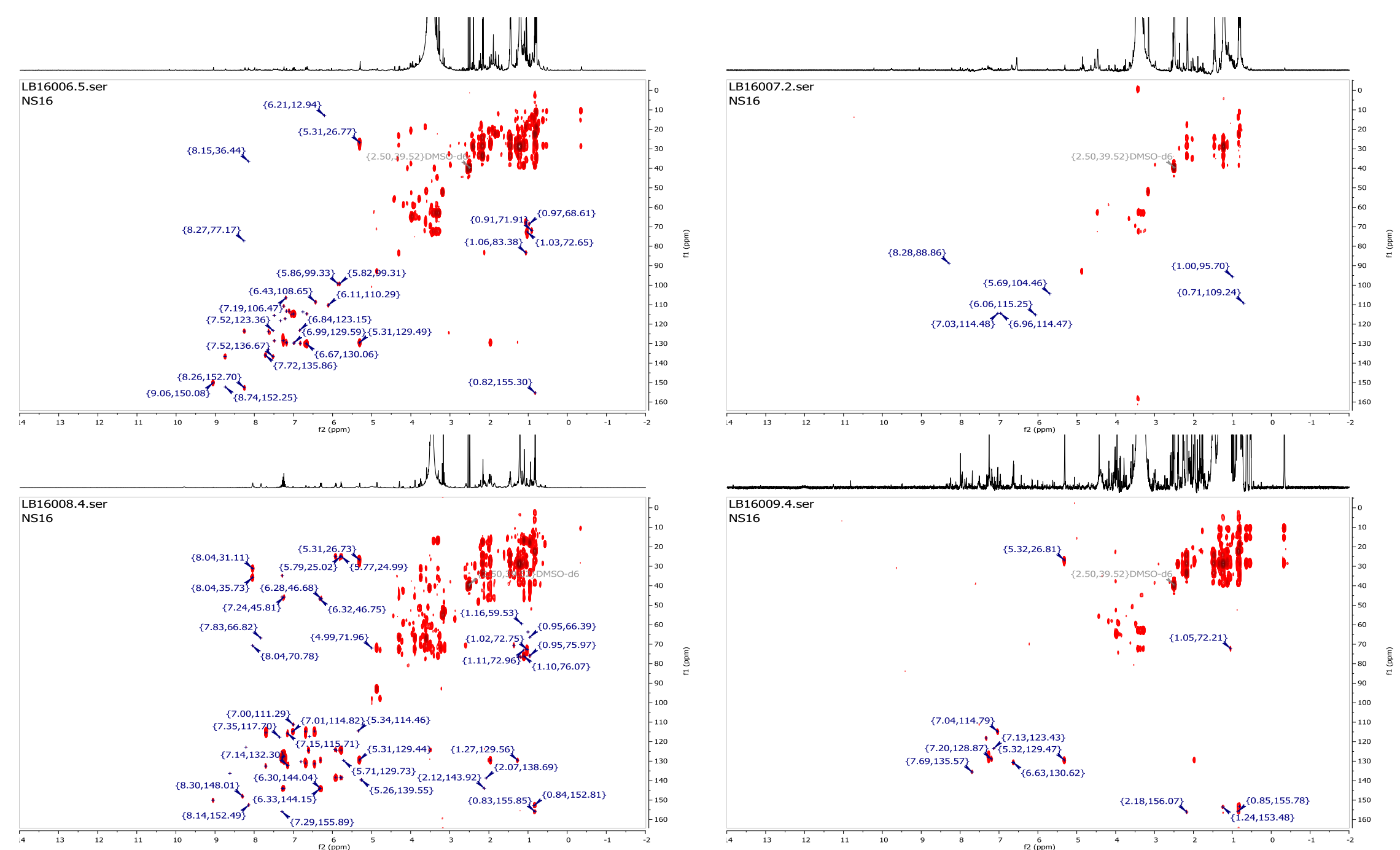

Figure IIA. HSQC-TOCSY NMR spectra of actinomycete crude extracts (continued) (DMSO- $d_{6 ;} 800 \mathrm{MHz}$; USC-isolate number given in the top left corner of spectra) 

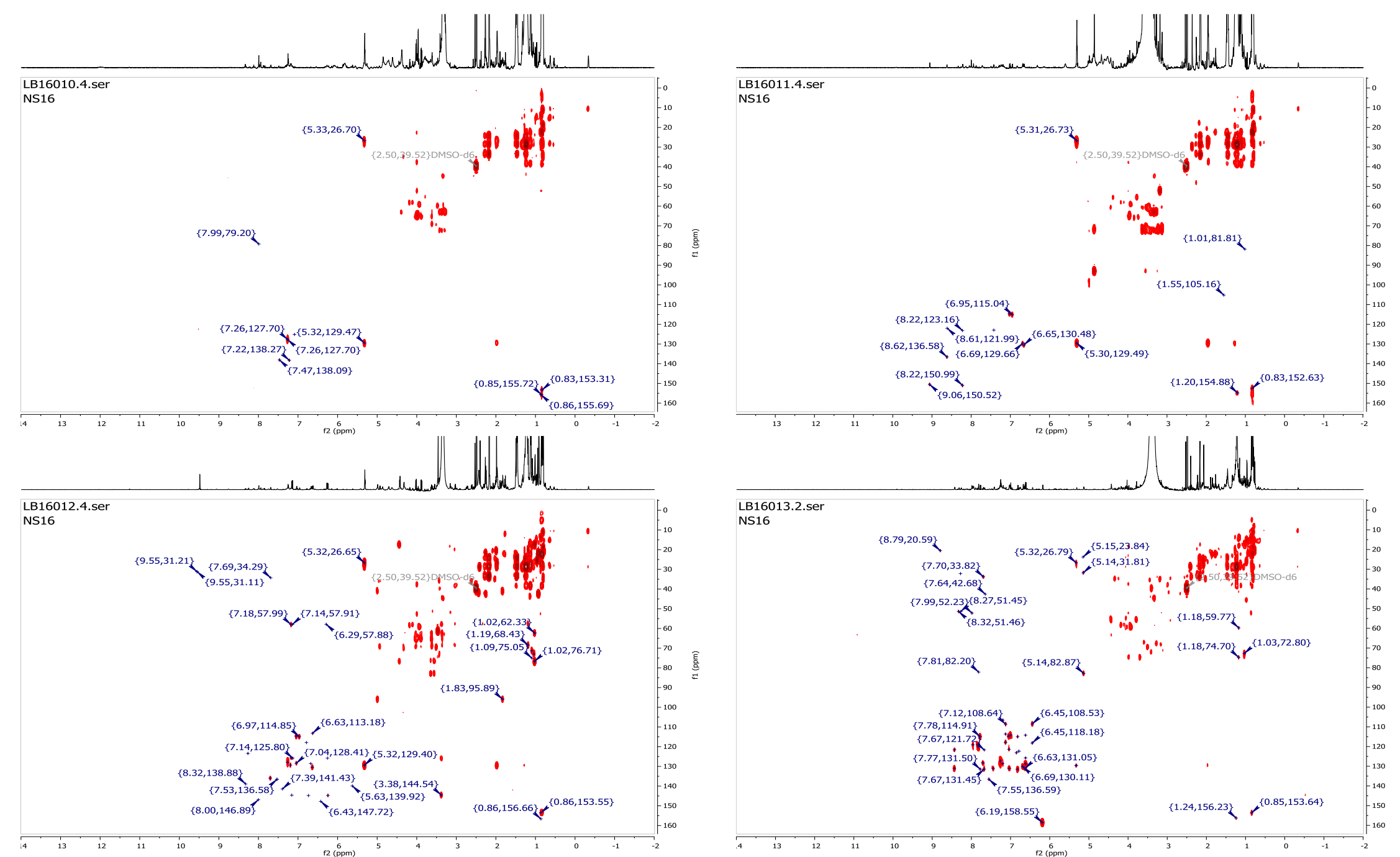

Figure IIA. HSQC-TOCSY NMR spectra of actinomycete crude extracts (continued) (DMSO- $d_{6 ;} 800 \mathrm{MHz}$; USC-isolate number given in the top left corner of spectra) 


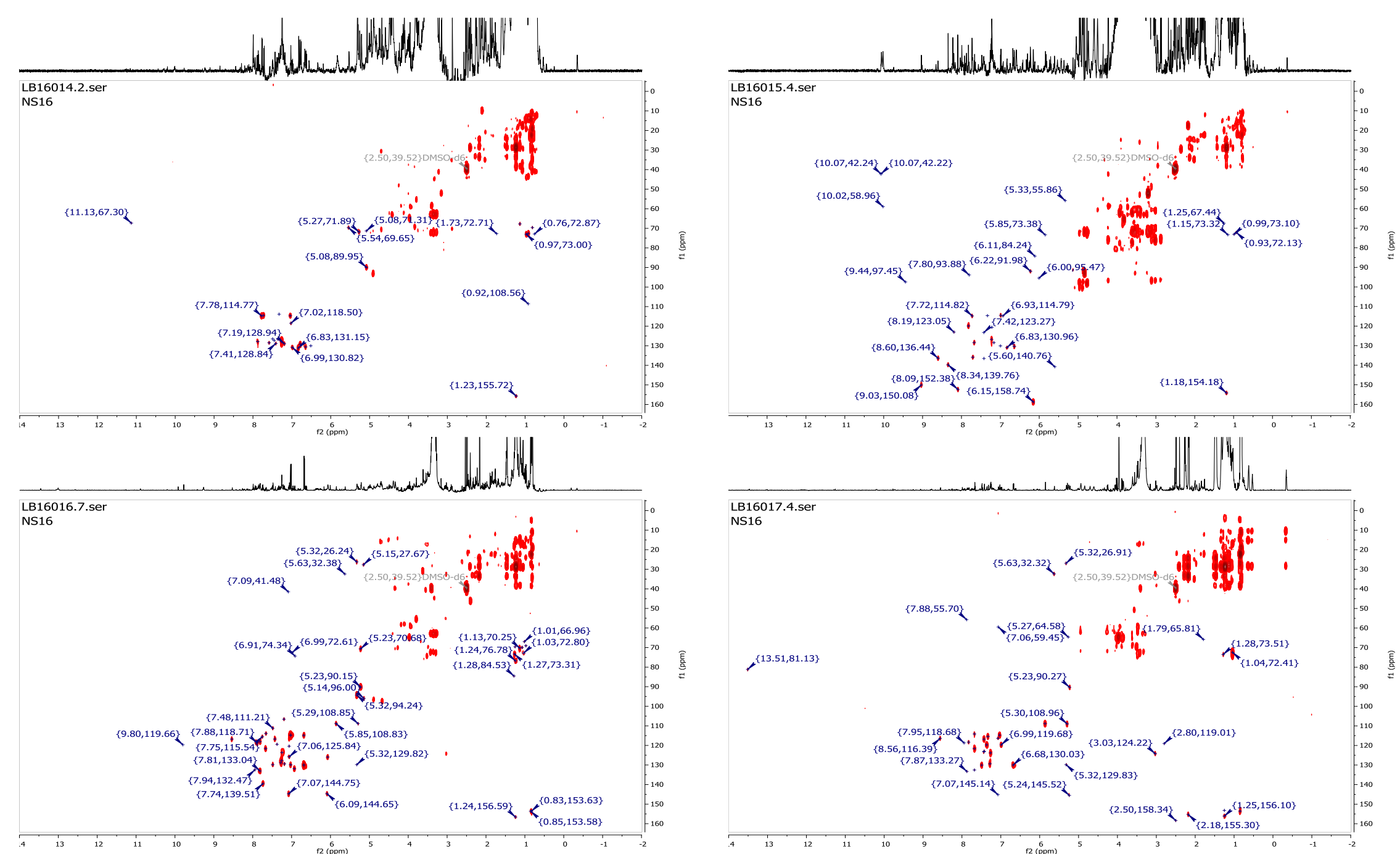

Figure IIA. HSQC-TOCSY NMR spectra of actinomycete crude extracts (continued) (DMSO- $d_{6}$; $800 \mathrm{MHz}$; USC-isolate number given in the top left corner of spectra) 

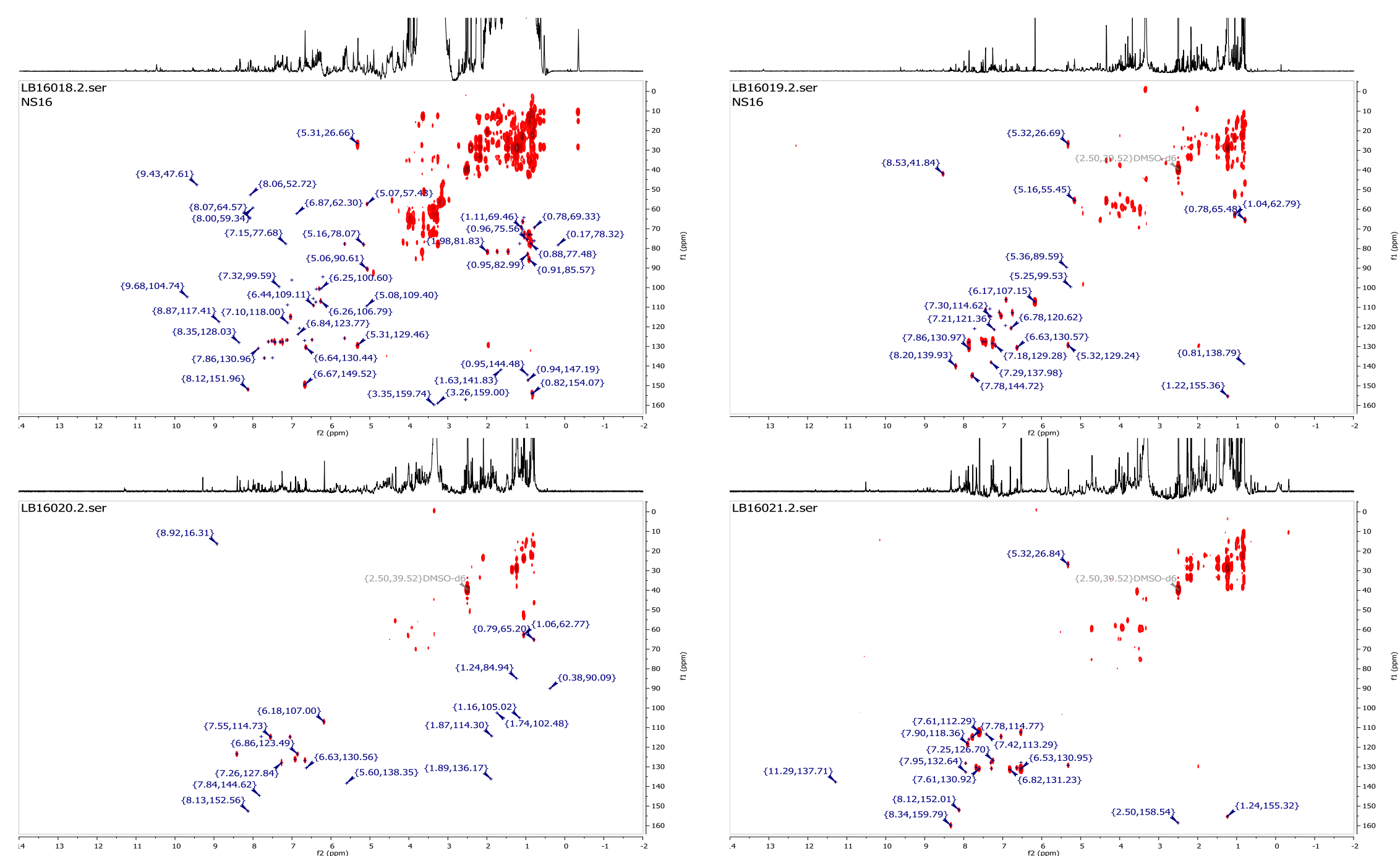

Figure IIA. HSQC-TOCSY NMR spectra of actinomycete crude extracts (continued) (DMSO- $d_{6 ;} 800 \mathrm{MHz}$; USC-isolate number given in the top left corner of spectra) 

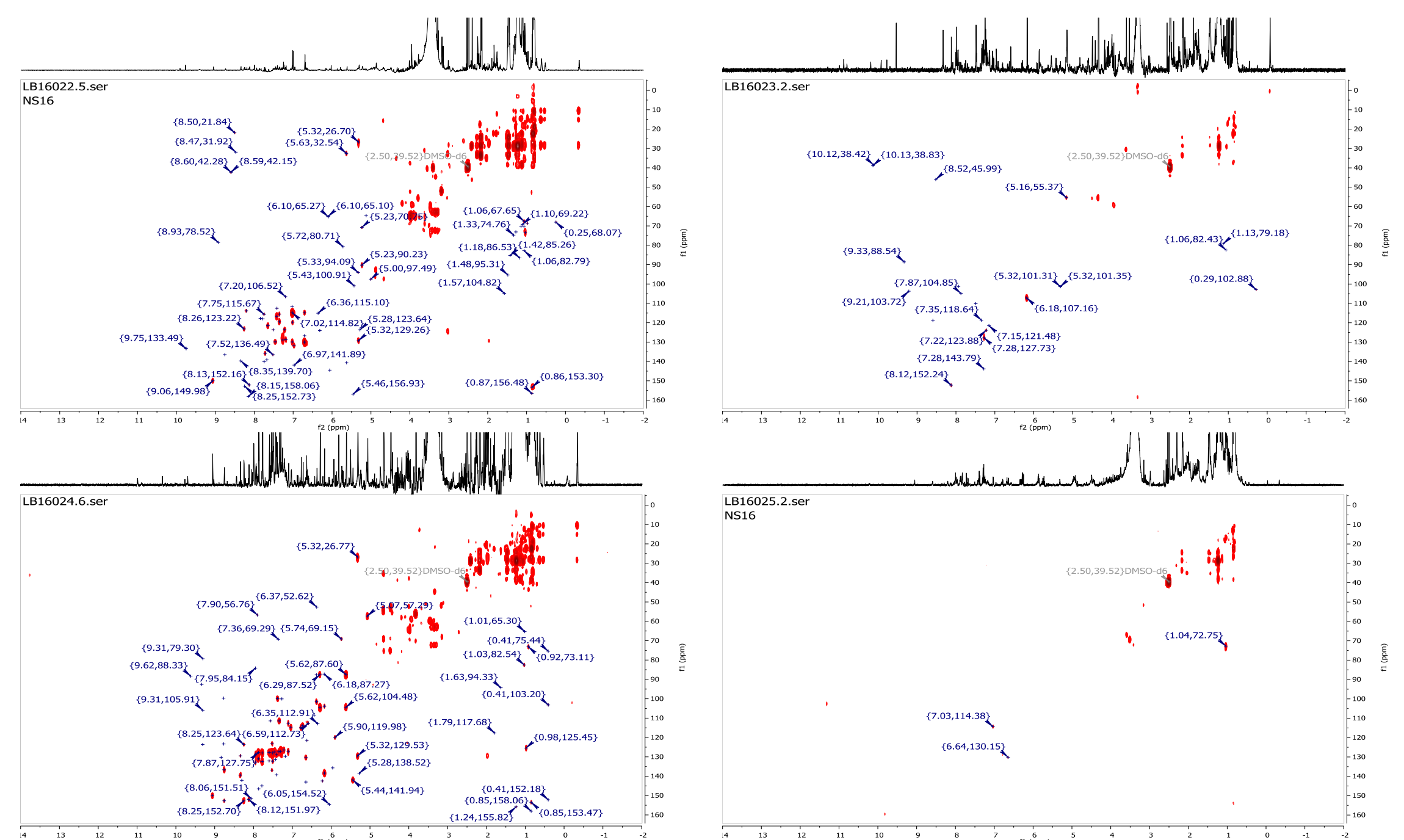

Figure IIA. HSQC-TOCSY NMR spectra of actinomycete crude extracts (continued) (DMSO- $d_{6 ;}$; $800 \mathrm{MHz}$; USC-isolate number given in the top left corner of spectra) 

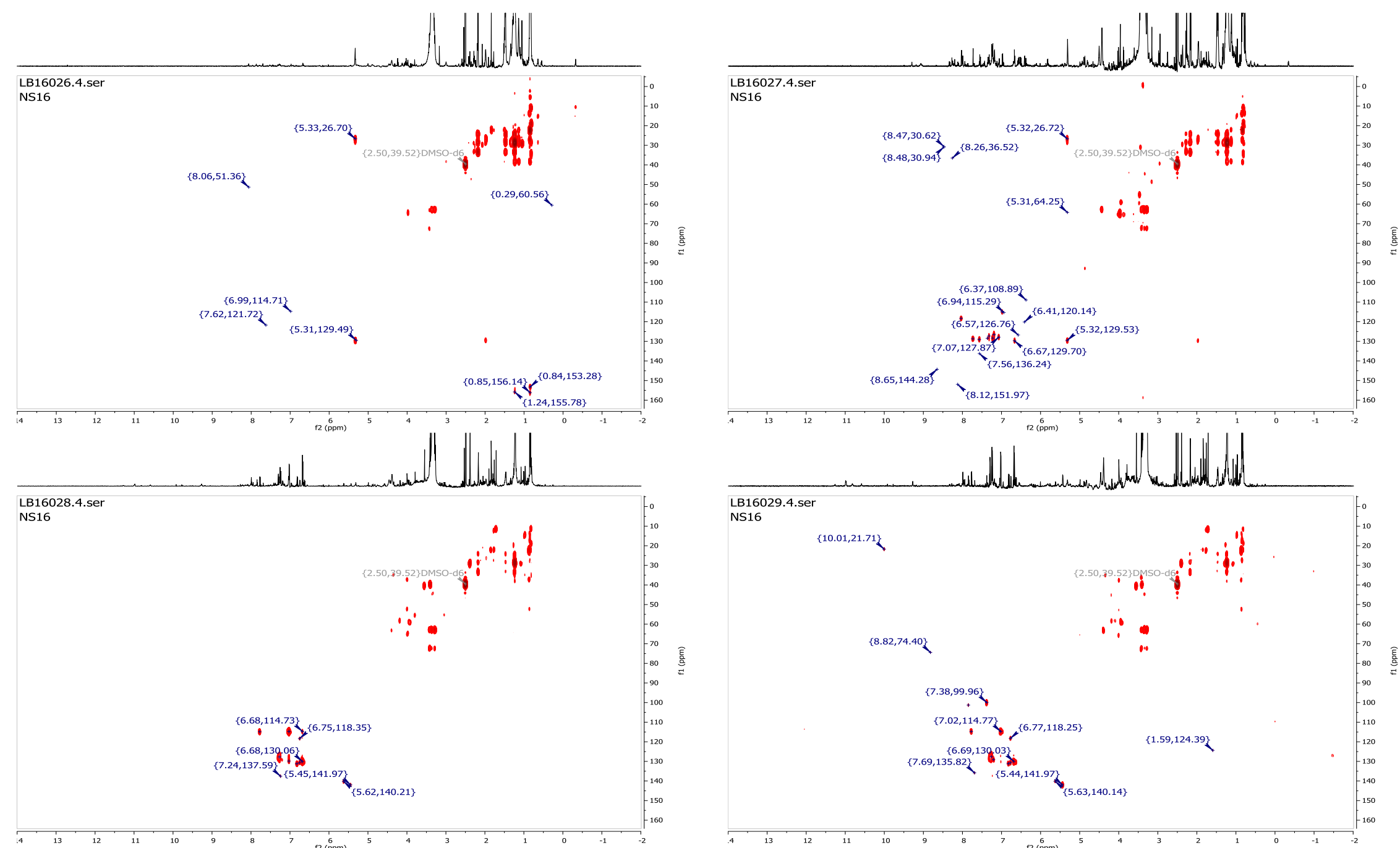

Figure IIA. HSQC-TOCSY NMR spectra of actinomycete crude extracts (continued) (DMSO- $d_{6}$; $800 \mathrm{MHz}$; USC-isolate number given in the top left corner of spectra) 

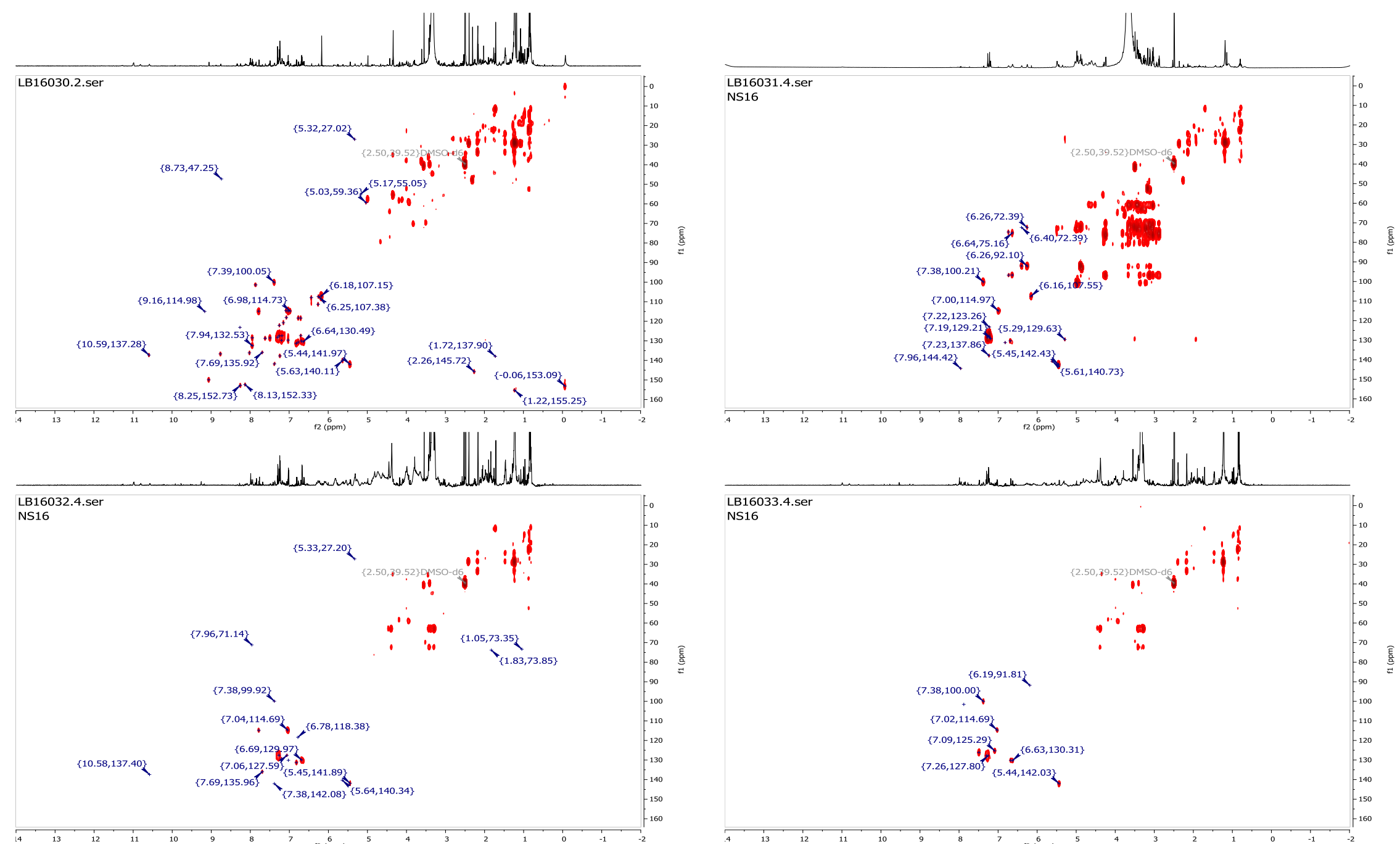

Figure IIA. HSQC-TOCSY NMR spectra of actinomycete crude extracts (continued) (DMSO- $d_{6}$; $800 \mathrm{MHz}$; USC-isolate number given in the top left corner of spectra) 

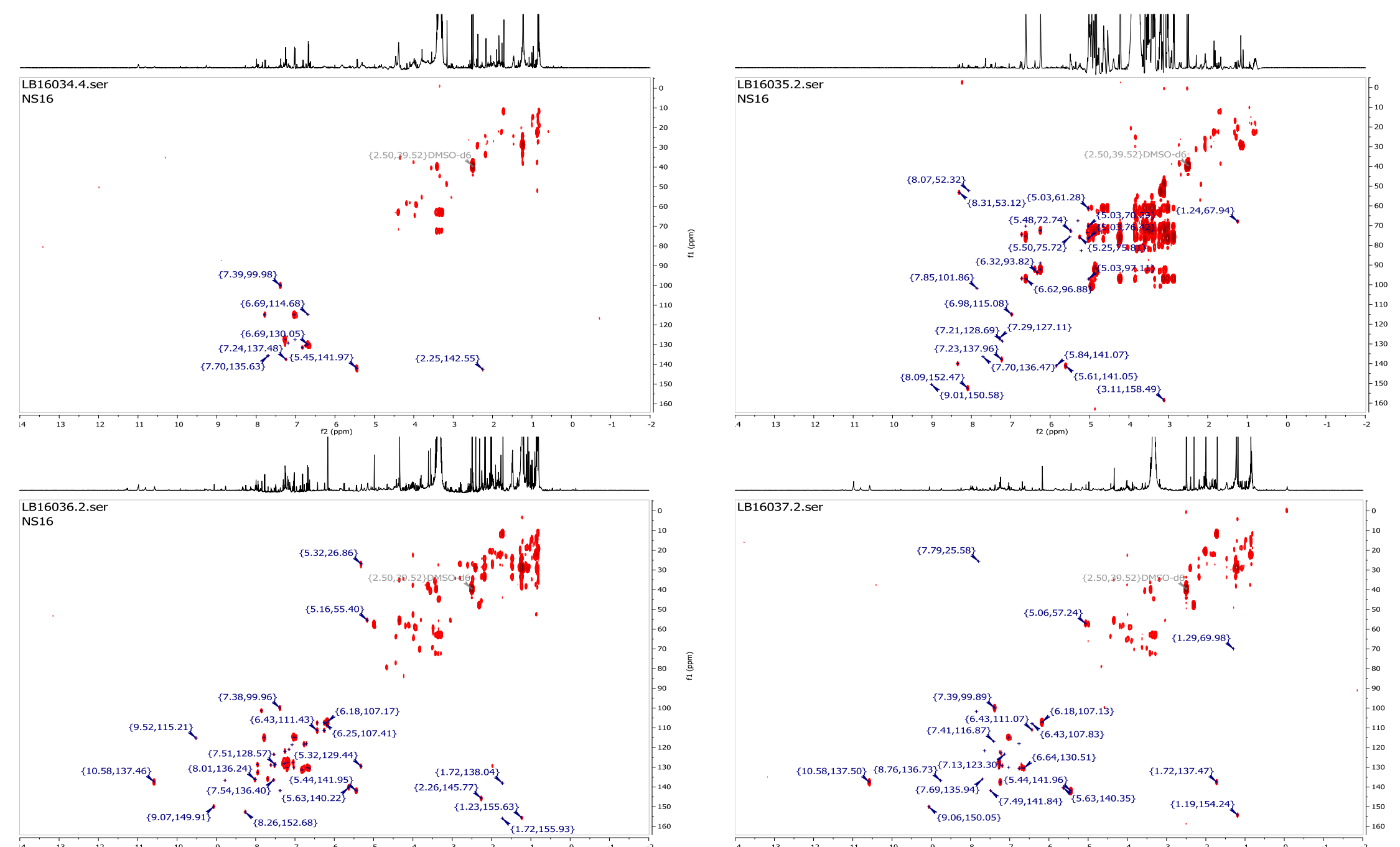

Figure IIA. HSQC-TOCSY NMR spectra of actinomycete crude extracts (continued) (DMSO- $d_{6}$; $800 \mathrm{MHz}$; USC-isolate number given in the top left corner of spectra) 


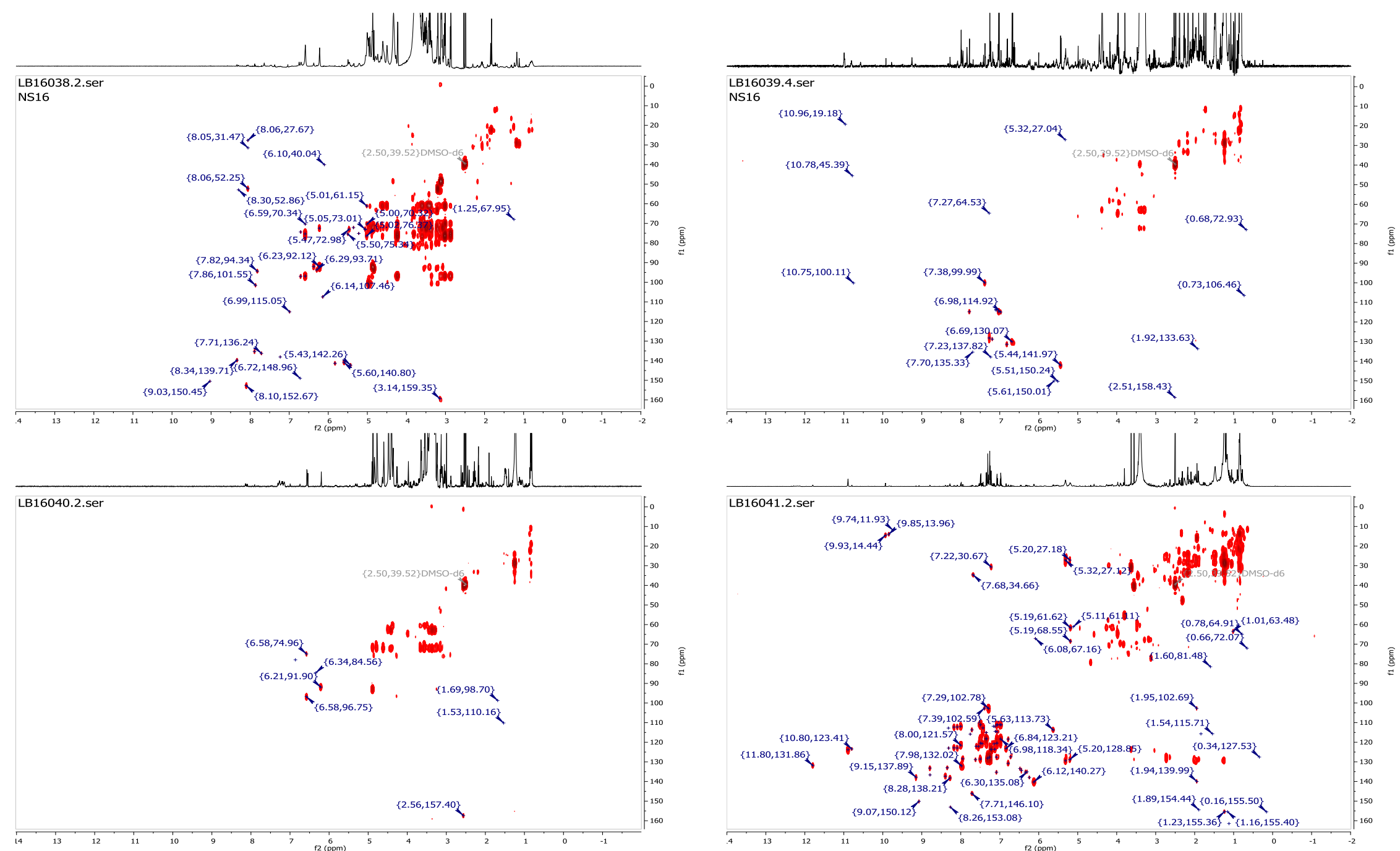

Figure IIA. HSQC-TOCSY NMR spectra of actinomycete crude extracts (continued) (DMSO- $d_{6}$; $800 \mathrm{MHz}$; USC-isolate number given in the top left corner of spectra) 

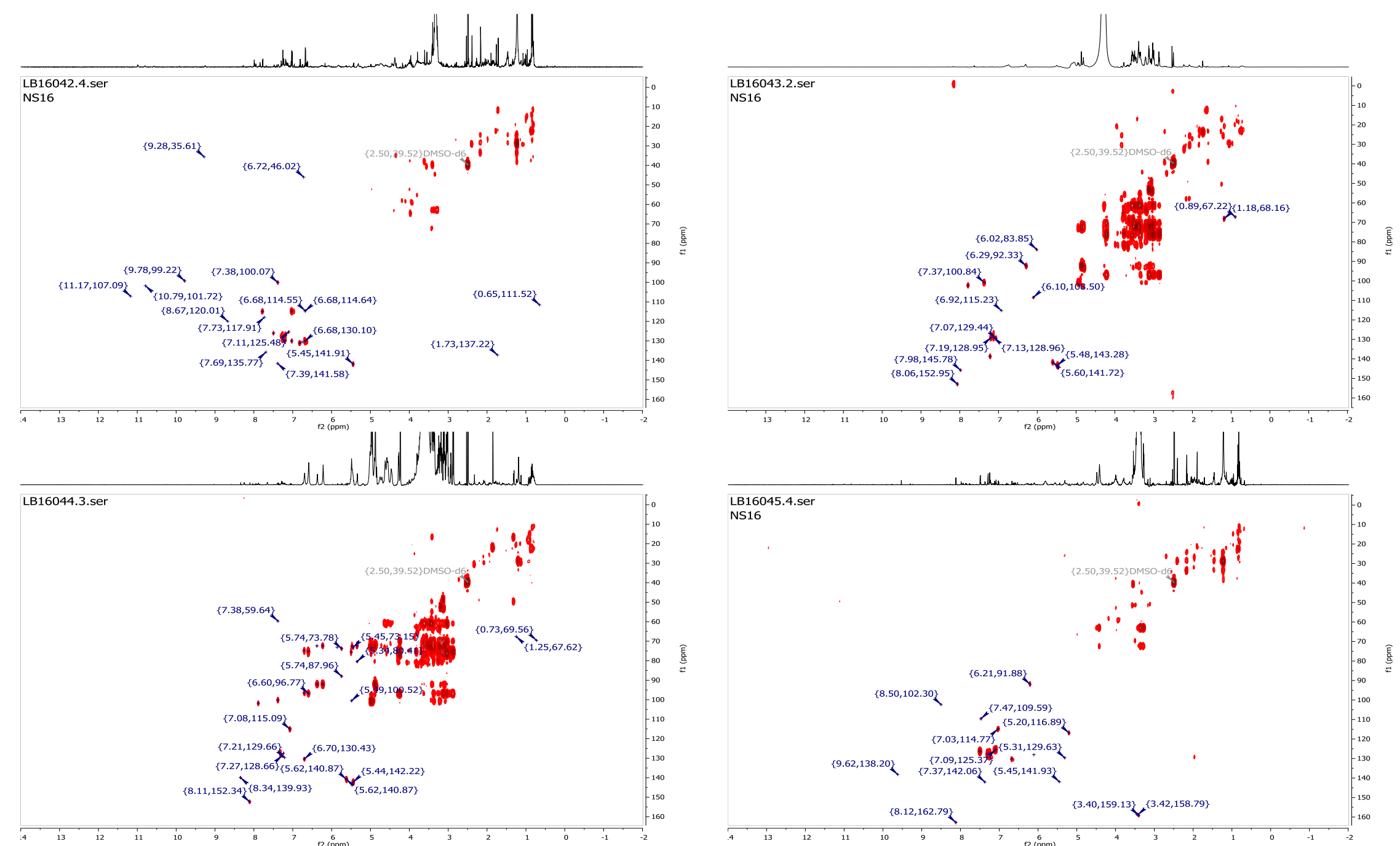

Figure IIA. HSQC-TOCSY NMR spectra of actinomycete crude extracts (continued) (DMSO- $d_{6} .800 \mathrm{MHz}$; USC-isolate number given in the top left corner of spectra) 

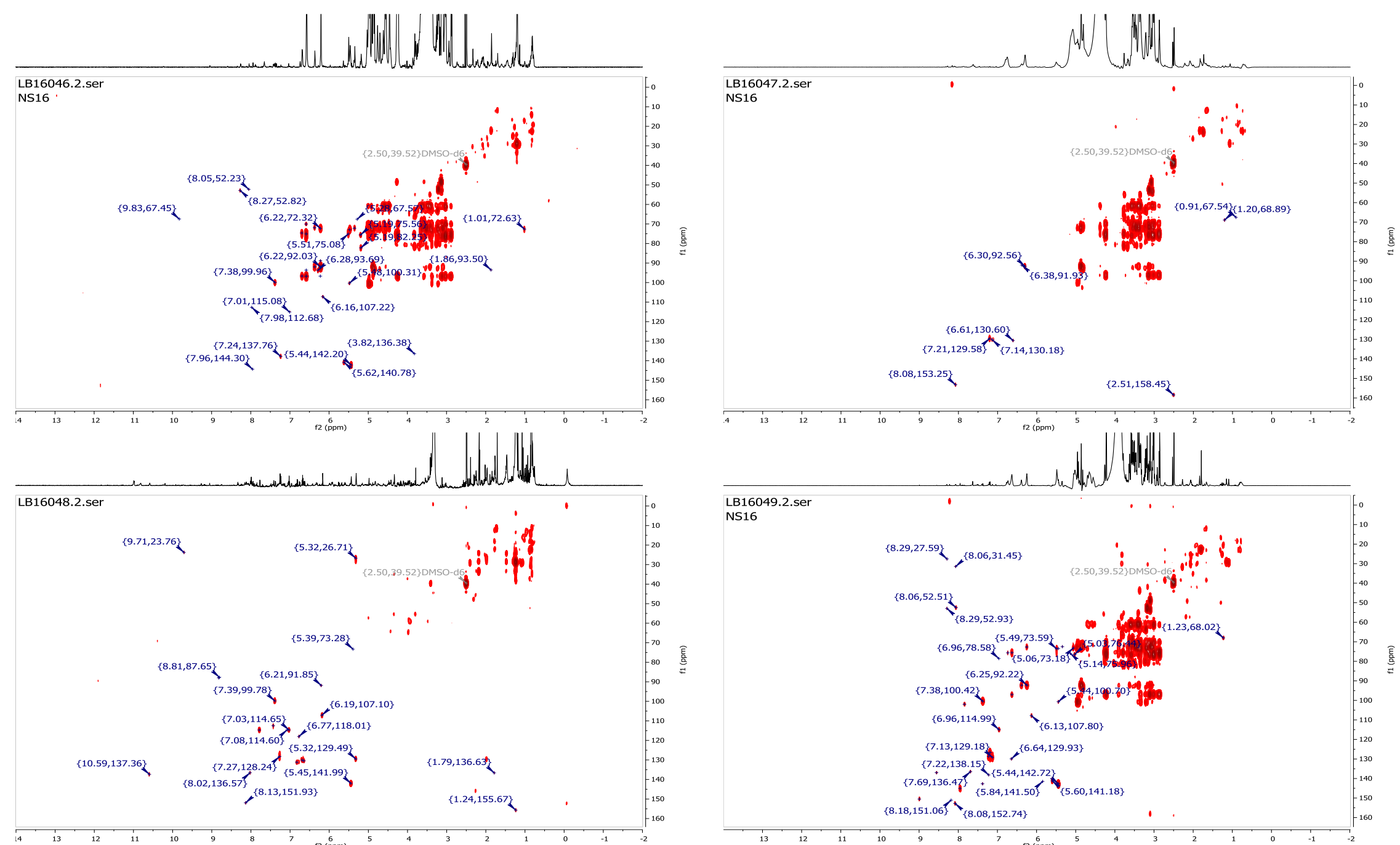

Figure IIA. HSQC-TOCSY NMR spectra of actinomycete crude extracts (continued) (DMSO- $d_{6}$; $800 \mathrm{MHz}$; USC-isolate number given in the top left corner of spectra) 


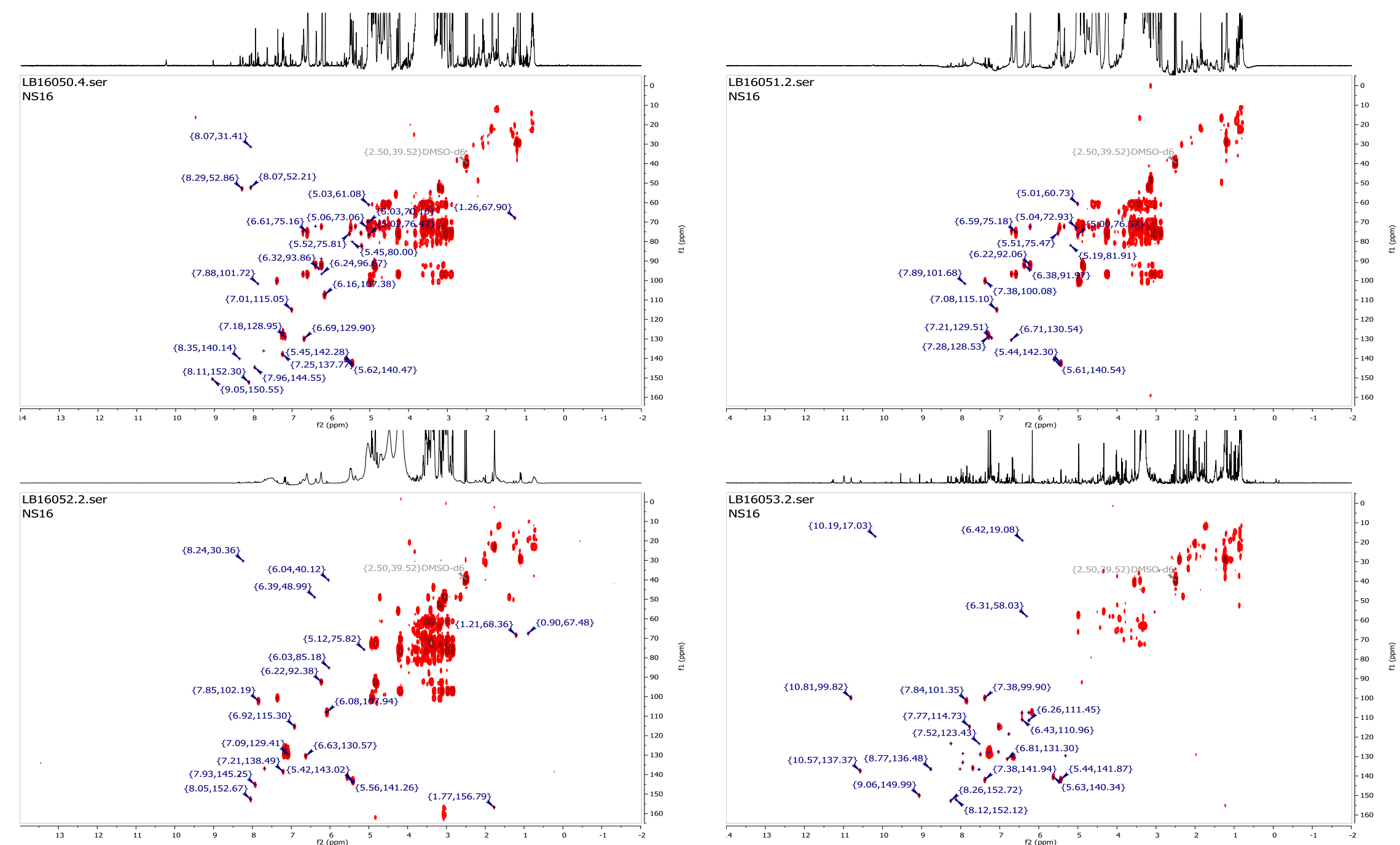

Figure IIA. HSQC-TOCSY NMR spectra of actinomycete crude extracts (continued) (DMSO- $d_{6}$; $800 \mathrm{MHz}$; USC-isolate number given in the top left corner of spectra) 

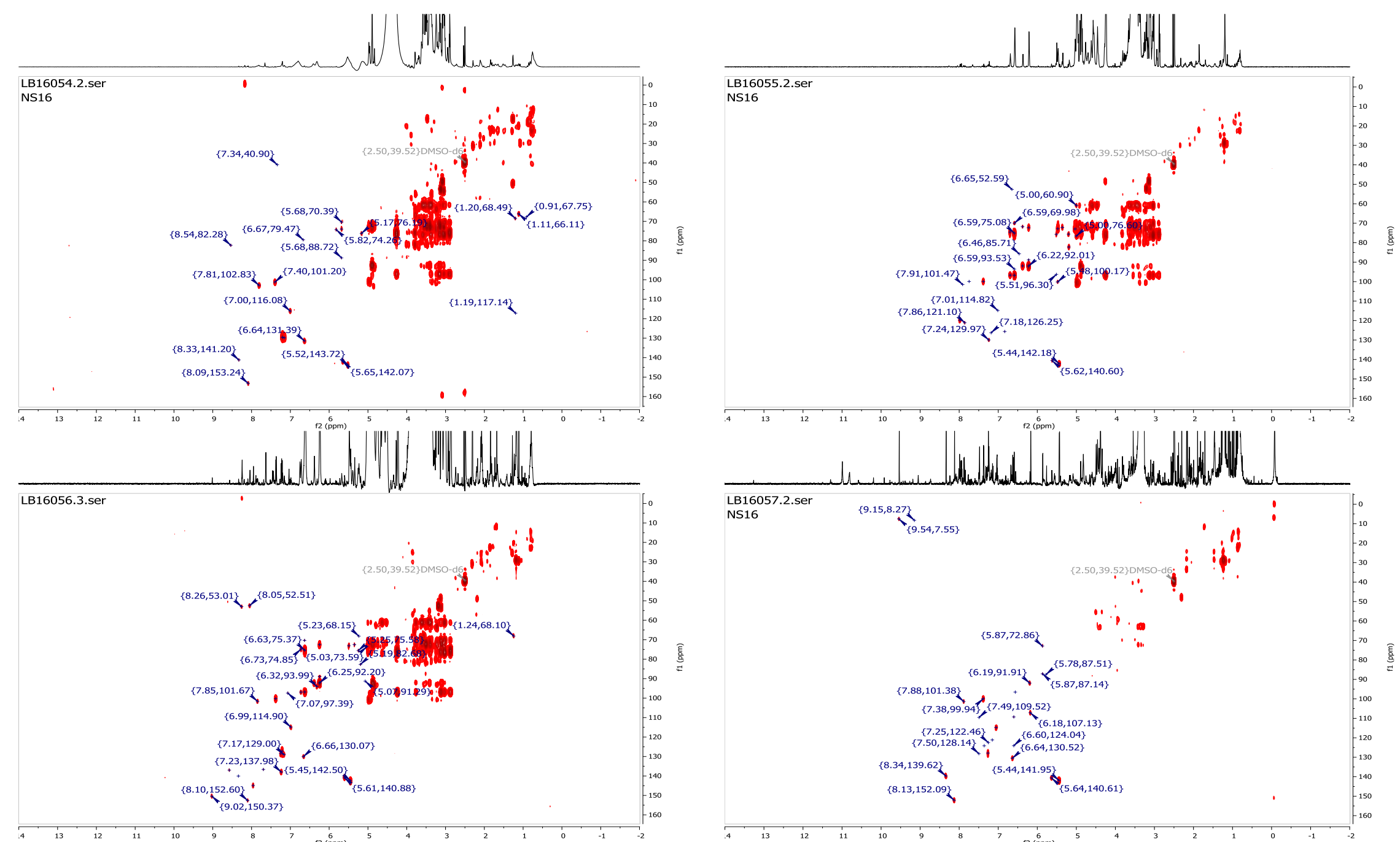

Figure IIA. HSQC-TOCSY NMR spectra of actinomycete crude extracts (continued) (DMSO- $d_{6}$. $800 \mathrm{MHz}$; USC-isolate number given in the top left corner of spectra) 

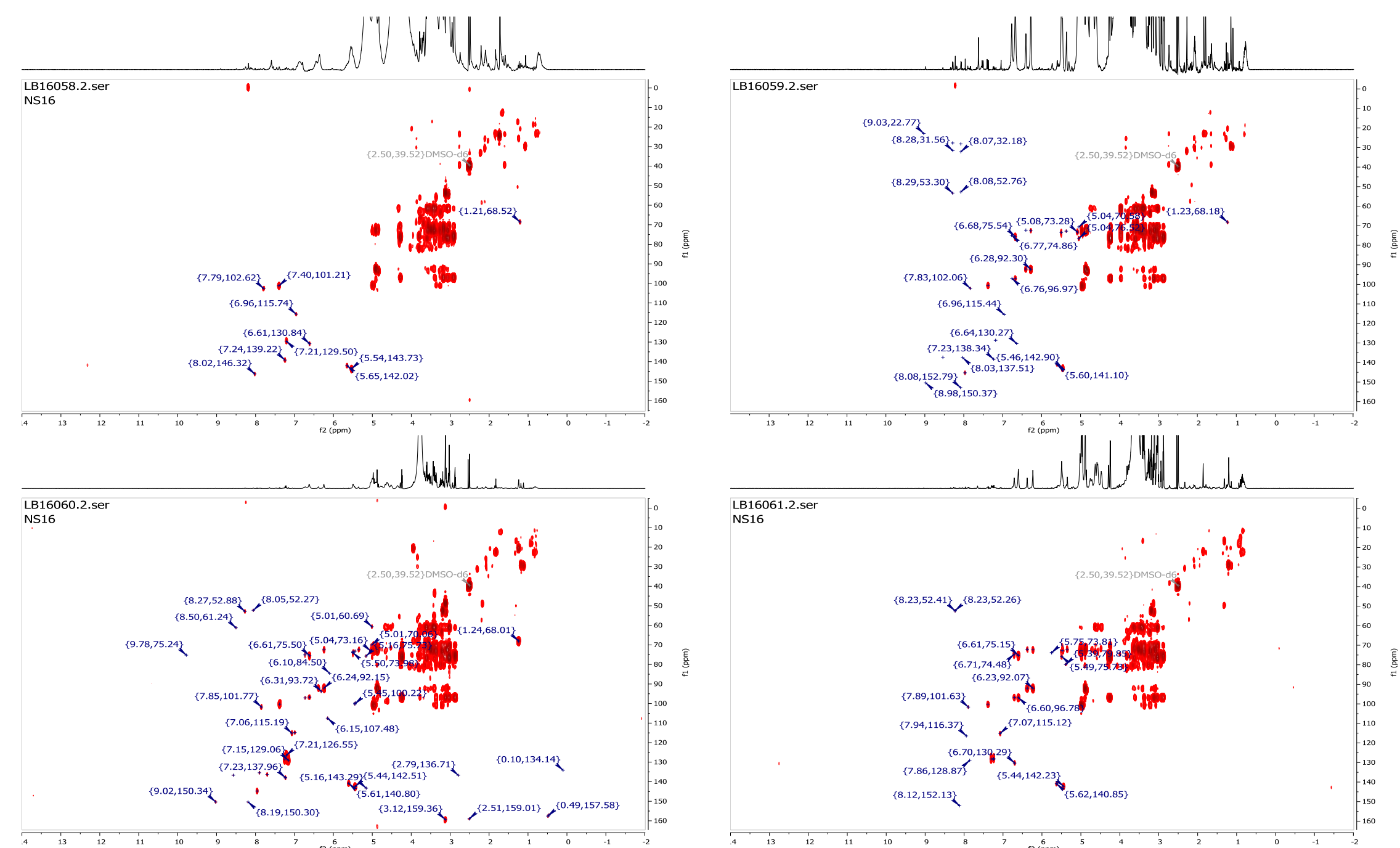

Figure IIA. HSQC-TOCSY NMR spectra of actinomycete crude extracts (continued) (DMSO- $d_{6 ;} 800 \mathrm{MHz}$; USC-isolate number given in the top left corner of spectra) 

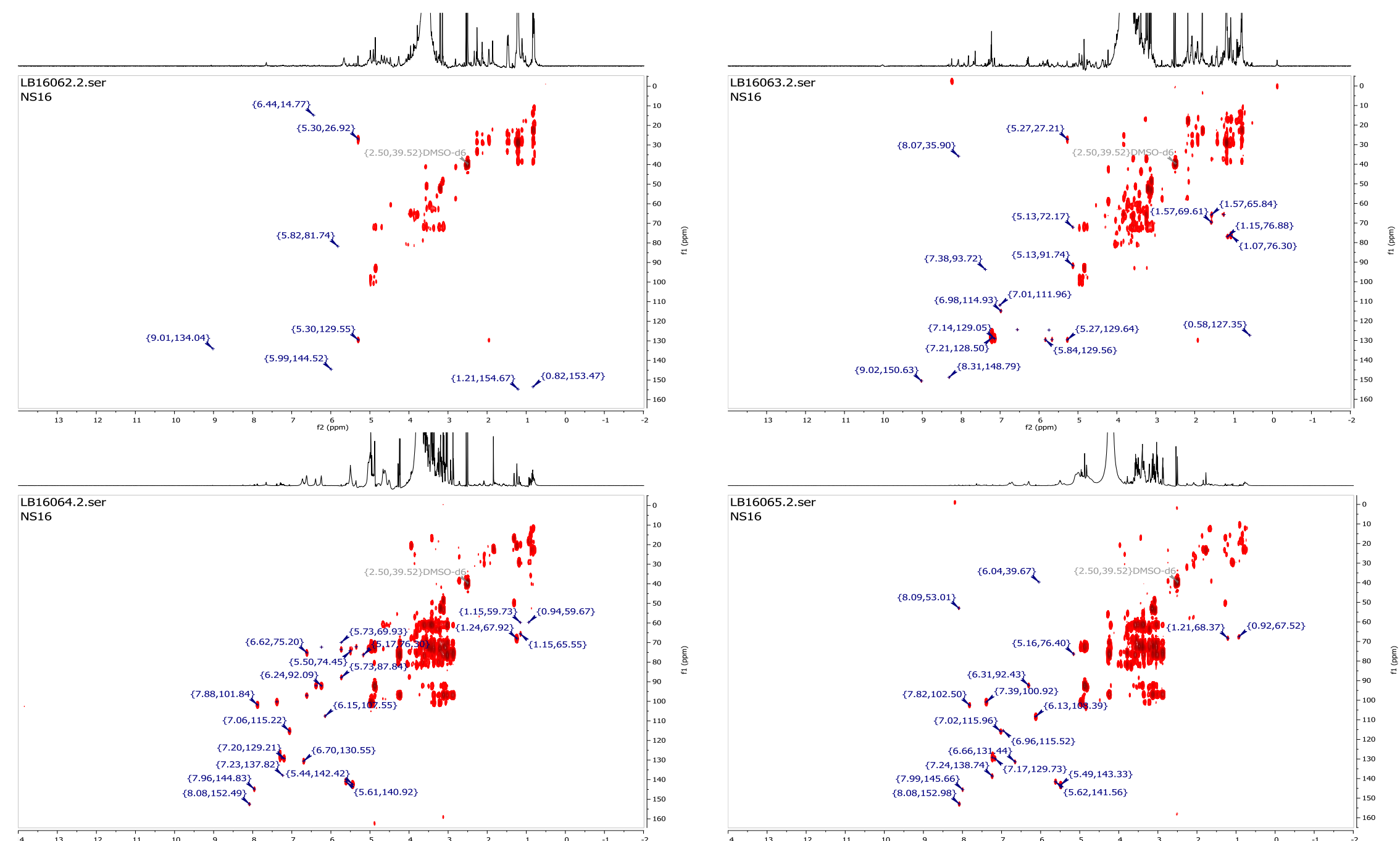

Figure IIA. HSQC-TOCSY NMR spectra of actinomycete crude extracts (continued) (DMSO- $d_{6}$ : $800 \mathrm{MHz}$; USC-isolate number given in the top left corner of spectra) 

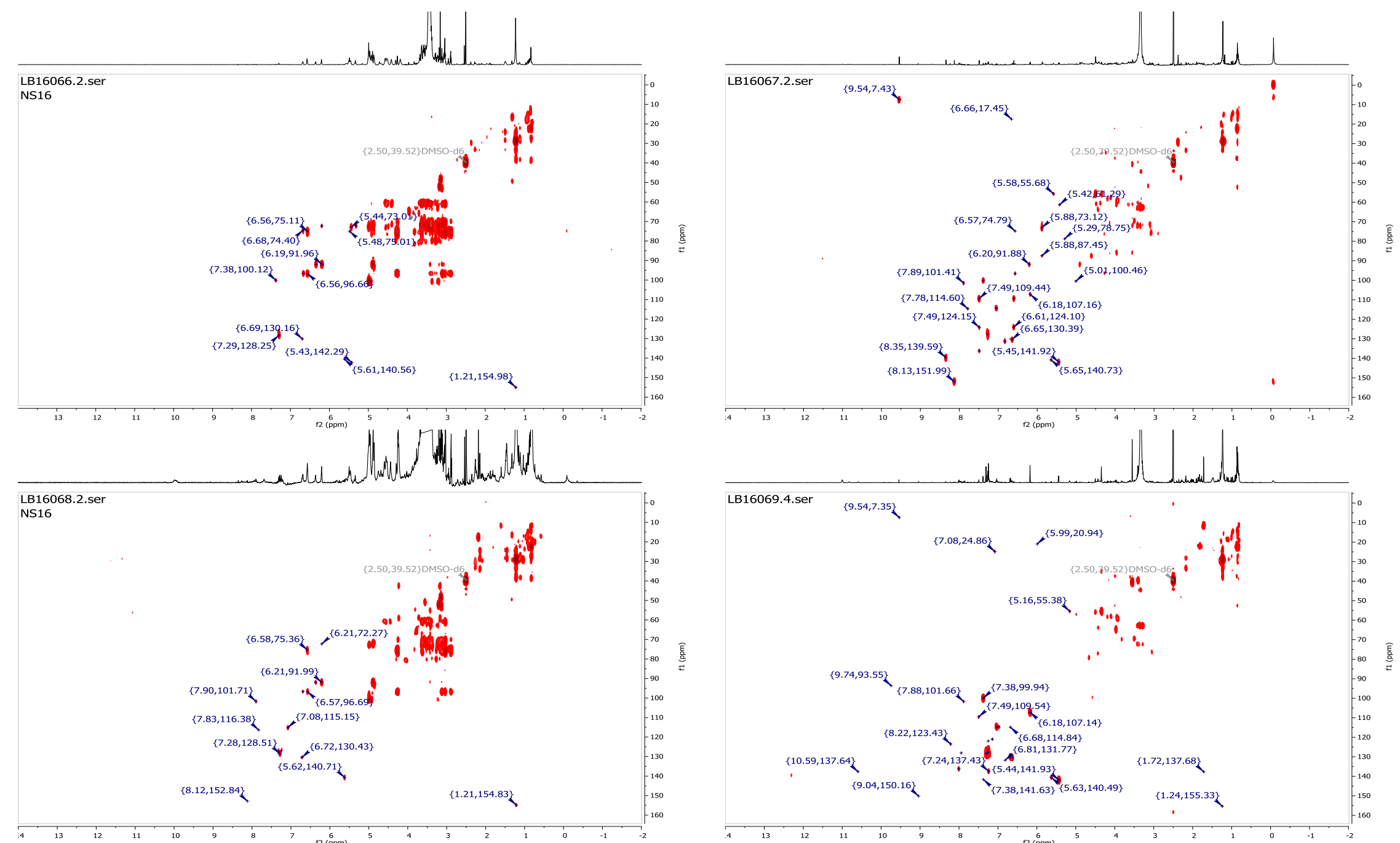

Figure IIA. HSQC-TOCSY NMR spectra of actinomycete crude extracts (continued) (DMSO- $d_{6}$; $800 \mathrm{MHz}$; USC-isolate number given in the top left corner of spectra) 


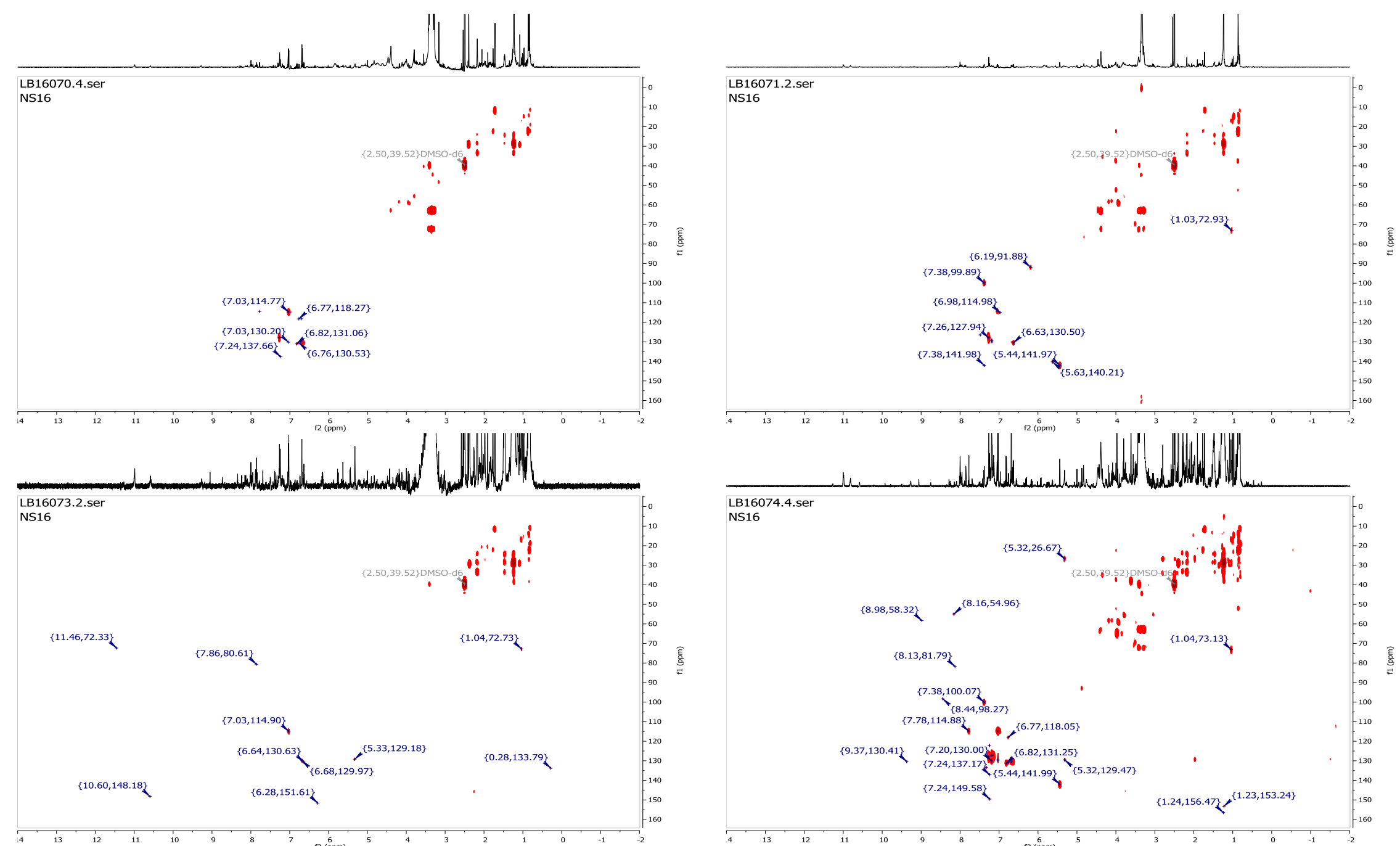

Figure IIA. HSQC-TOCSY NMR spectra of actinomycete crude extracts (continued) (DMSO- $d_{6}$ : $800 \mathrm{MHz}$; USC-isolate number given in the top left corner of spectra) 


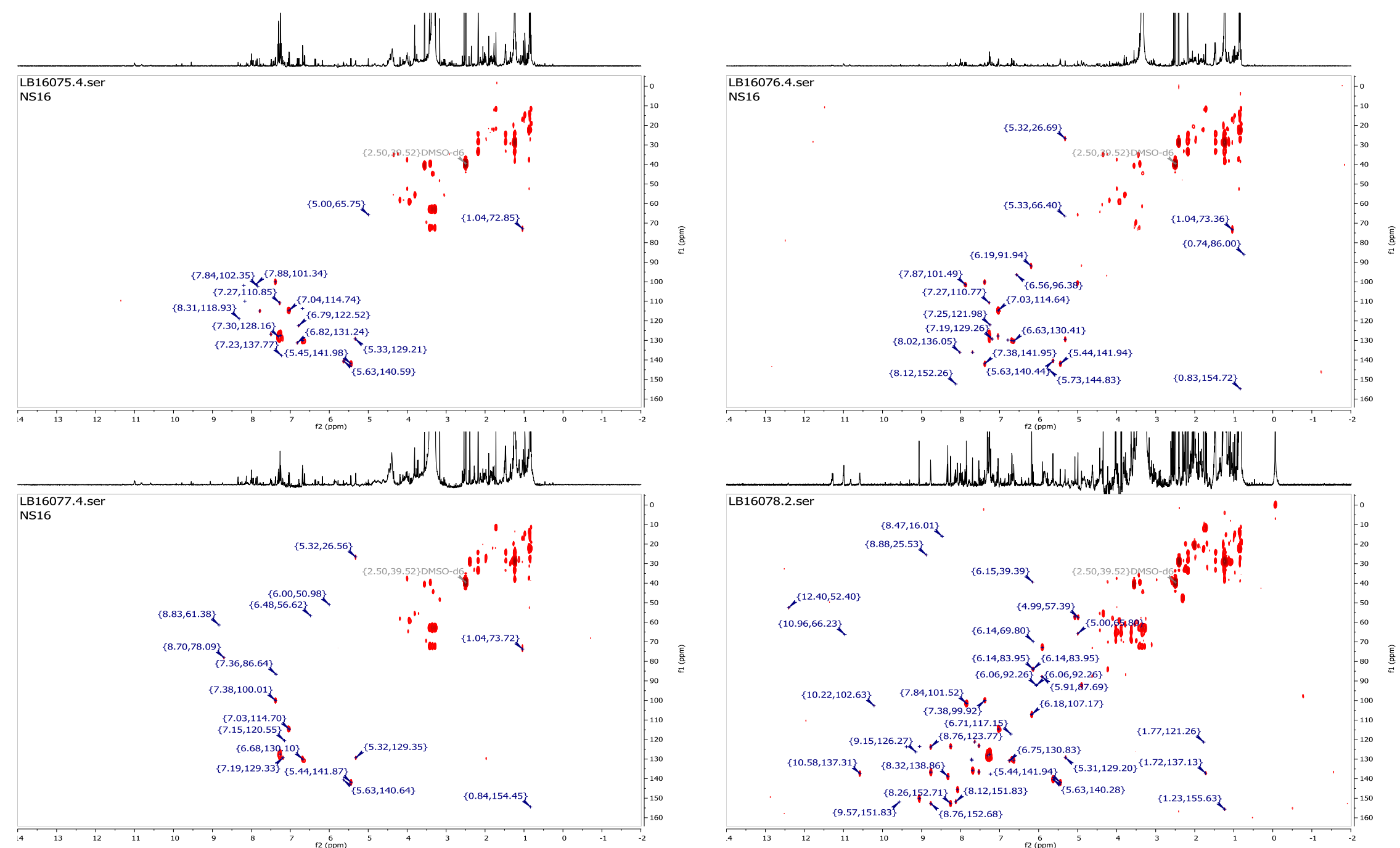

Figure IIA. HSQC-TOCSY NMR spectra of actinomycete crude extracts (continued) (DMSO- $d_{6}$ : $800 \mathrm{MHz}$; USC-isolate number given in the top left corner of spectra) 

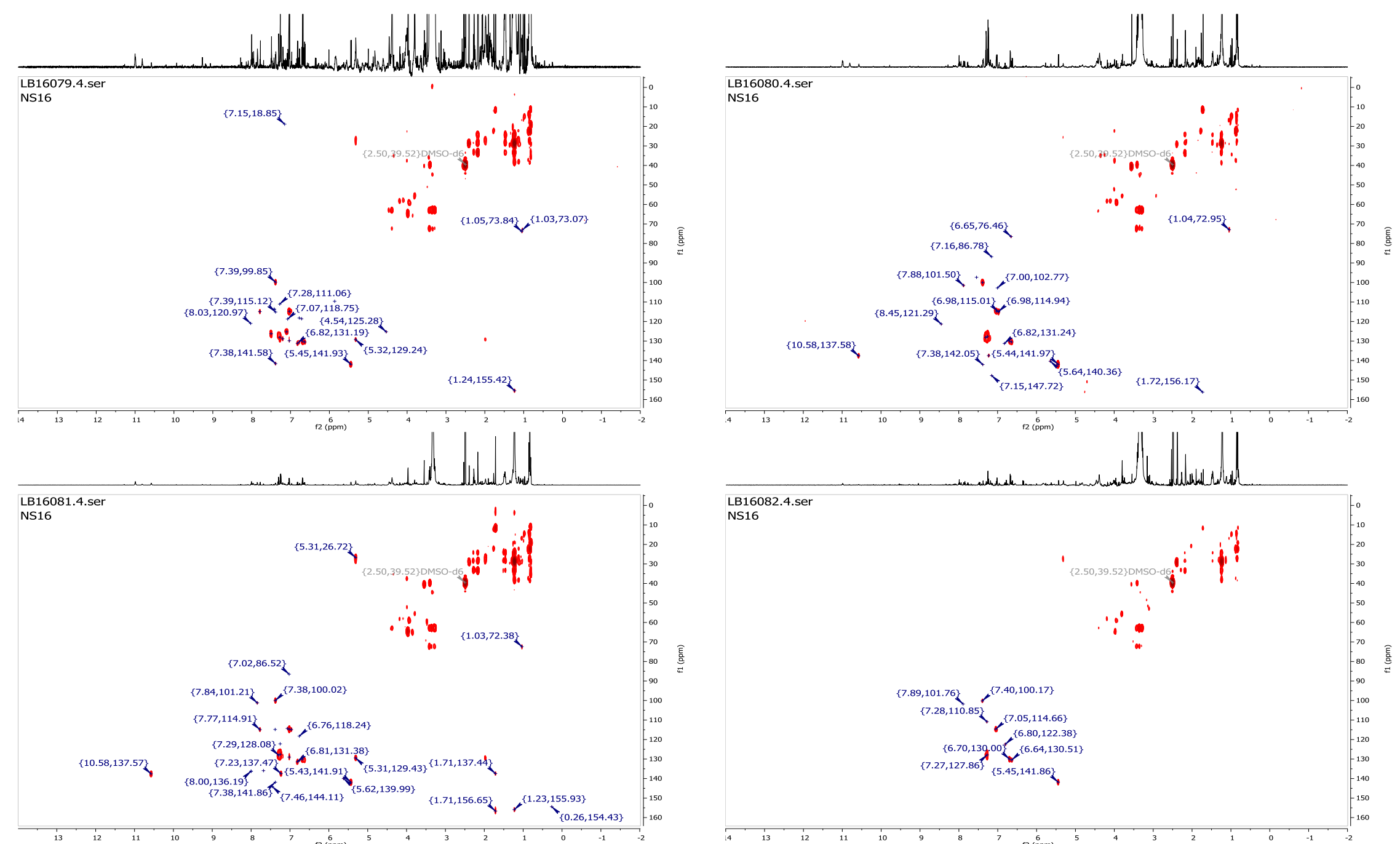

Figure IIA. HSQC-TOCSY NMR spectra of actinomycete crude extracts (continued) (DMSO- $d_{6 ;} 800 \mathrm{MHz}$; USC-isolate number given in the top left corner of spectra) 


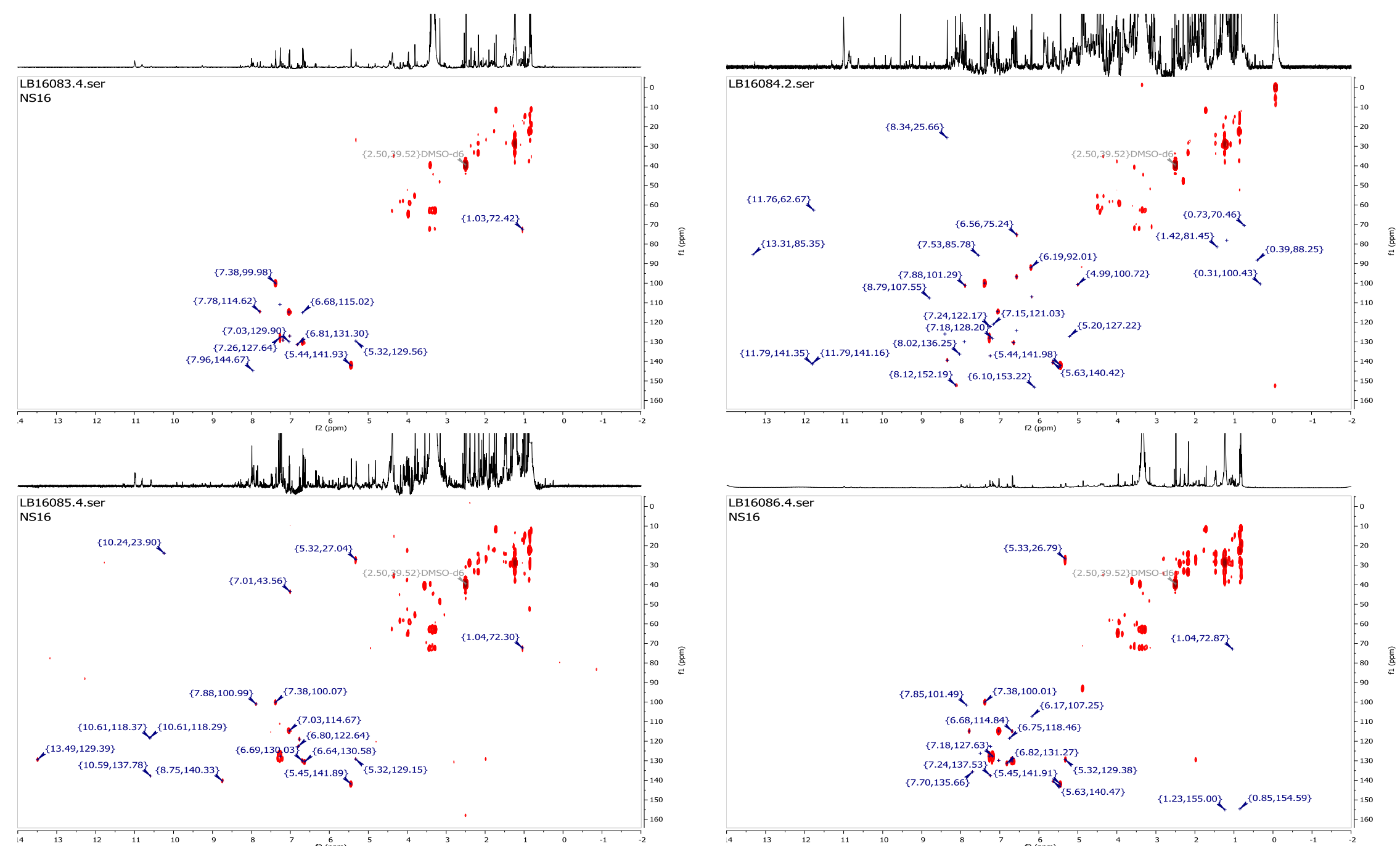

Figure IIA. HSQC-TOCSY NMR spectra of actinomycete crude extracts (continued) (DMSO- $d_{6}$; $800 \mathrm{MHz}$; USC-isolate number given in the top left corner of spectra) 

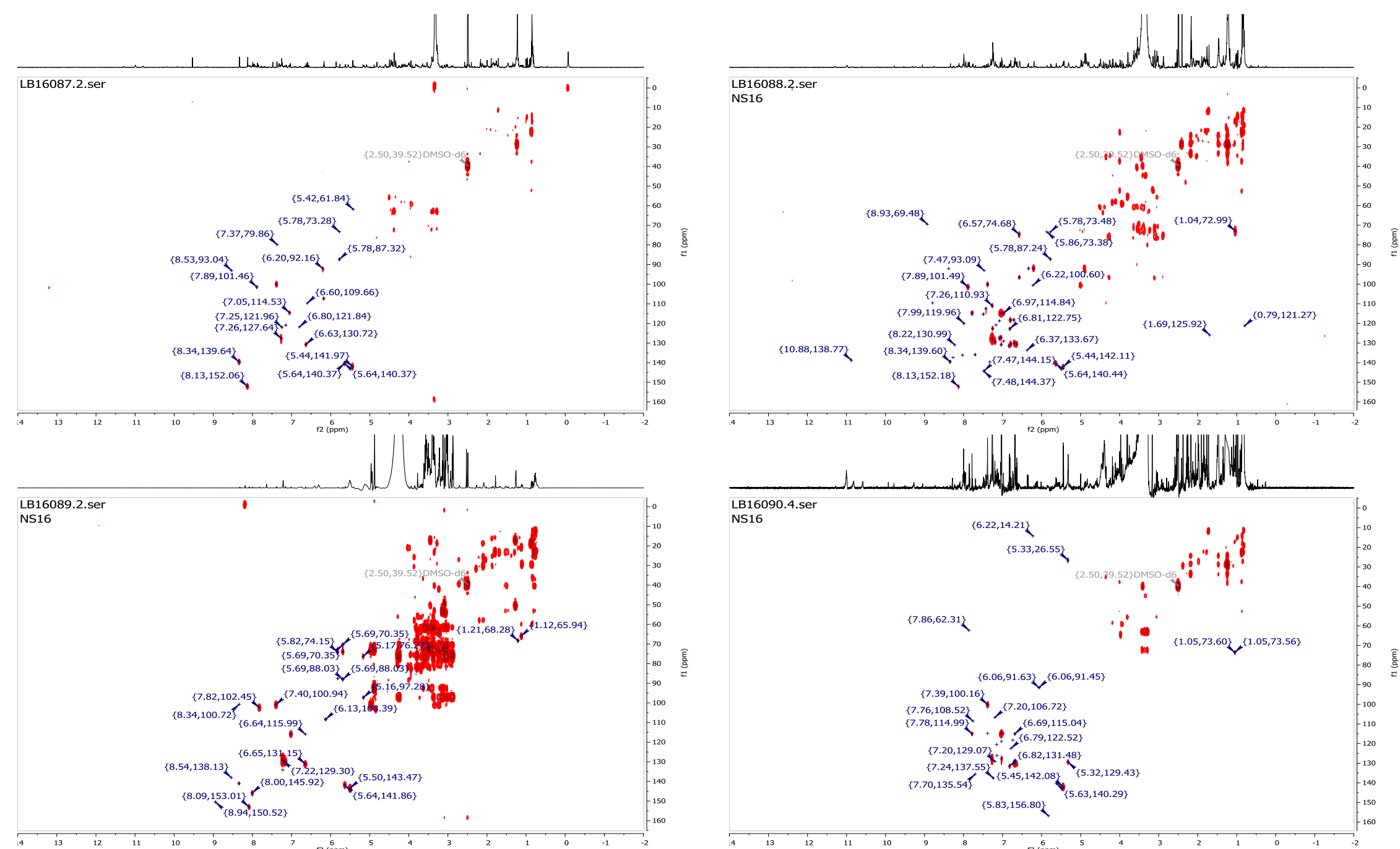

Figure IIA. HSQC-TOCSY NMR spectra of actinomycete crude extracts (continued) (DMSO- $d_{6}$. $800 \mathrm{MHz}$; USC-isolate number given in the top left corner of spectra) 

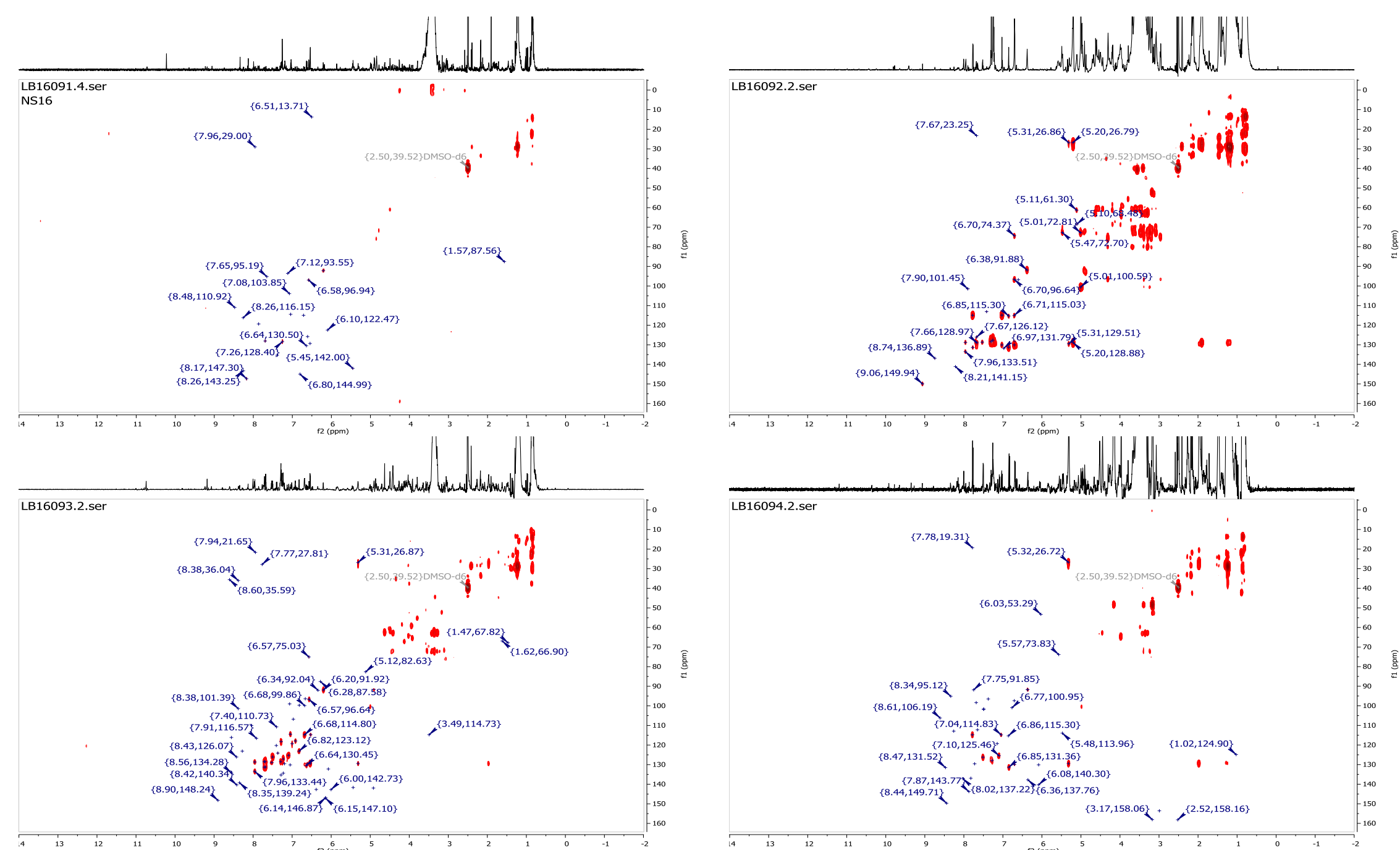

Figure IIA. HSQC-TOCSY NMR spectra of actinomycete crude extracts (continued) (DMSO- $d_{6 ;} 800 \mathrm{MHz}$; USC-isolate number given in the top left corner of spectra) 

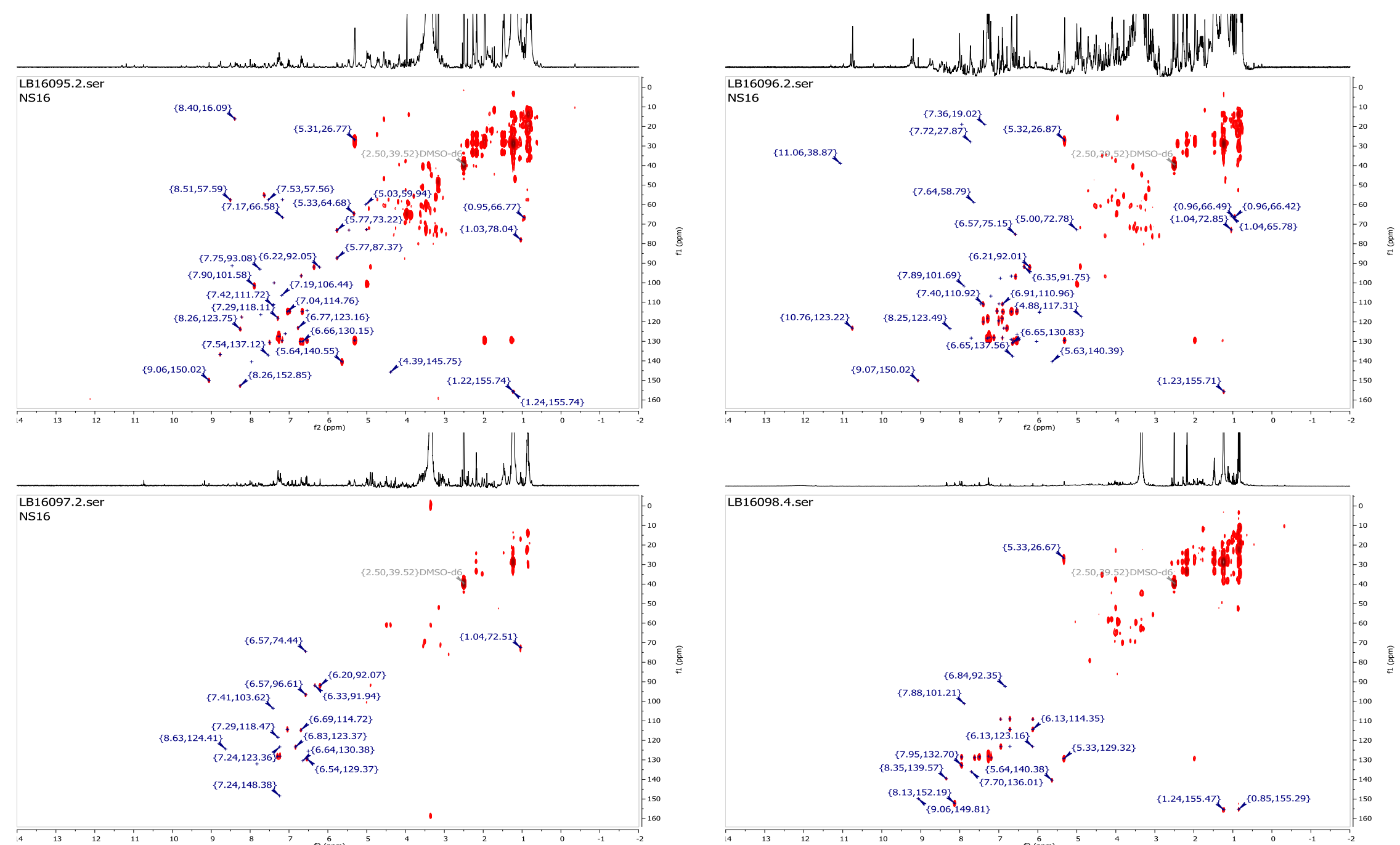

Figure IIA. HSQC-TOCSY NMR spectra of actinomycete crude extracts (continued) (DMSO- $d_{6}$ : $800 \mathrm{MHz}$; USC-isolate number given in the top left corner of spectra) 

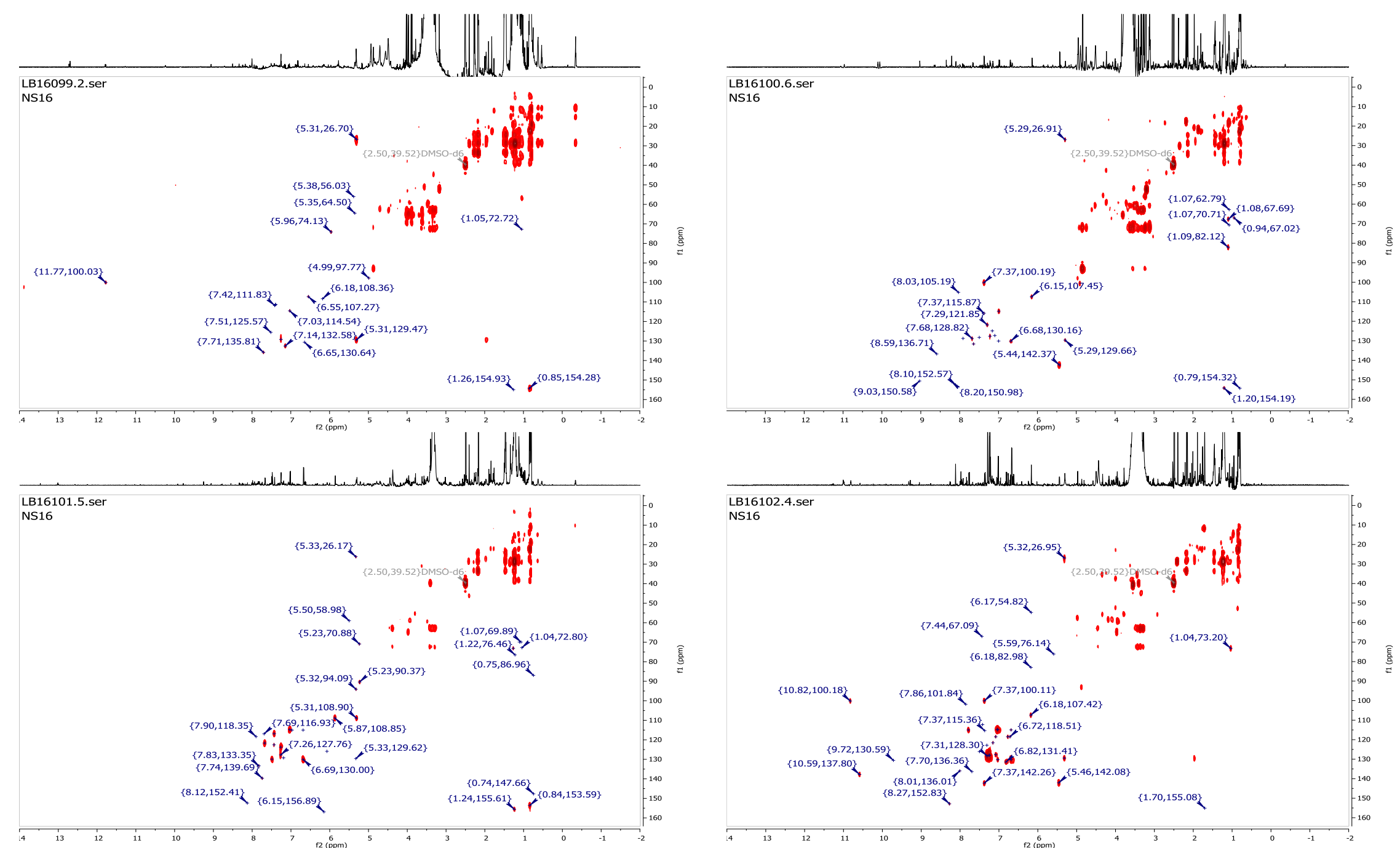

Figure IIA. HSQC-TOCSY NMR spectra of actinomycete crude extracts (continued) (DMSO- $d_{6}$ : $800 \mathrm{MHz}$; USC-isolate number given in the top left corner of spectra) 


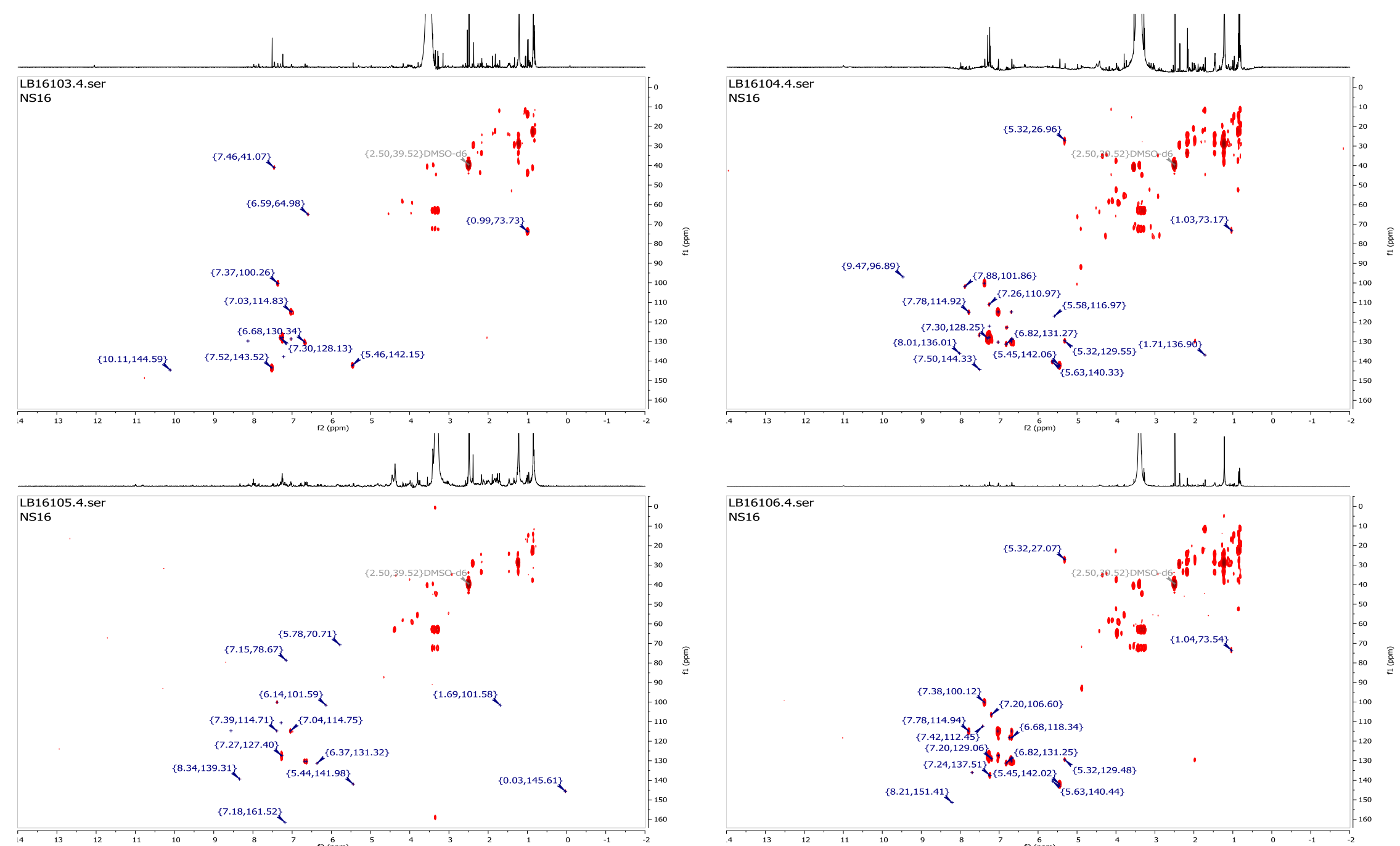

Figure IIA. HSQC-TOCSY NMR spectra of actinomycete crude extracts (continued) (DMSO- $d_{6}$ : $800 \mathrm{MHz}$; USC-isolate number given in the top left corner of spectra) 


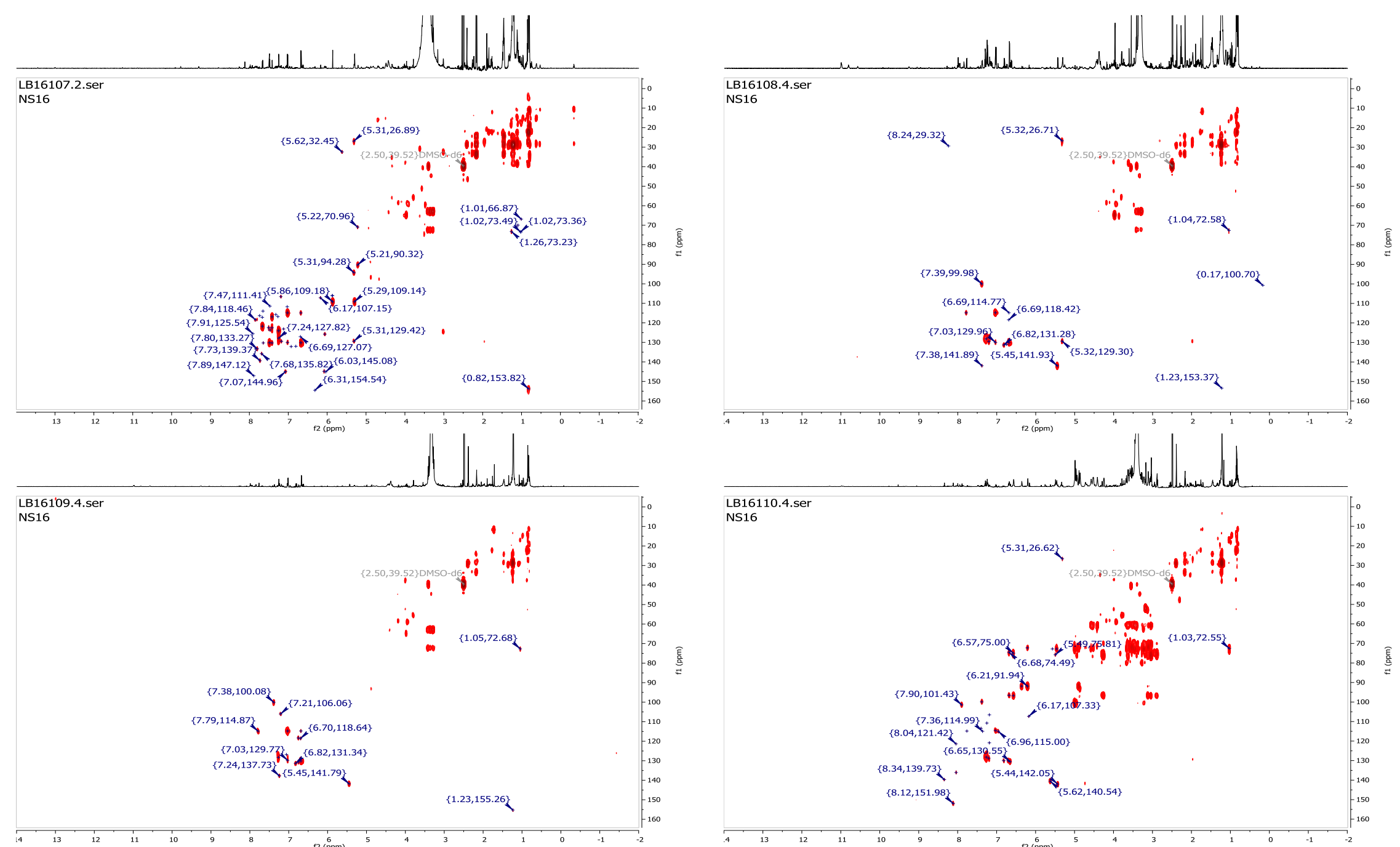

Figure IIA. HSQC-TOCSY NMR spectra of actinomycete crude extracts (continued) (DMSO- $d_{6}$ : $800 \mathrm{MHz}$; USC-isolate number given in the top left corner of spectra) 

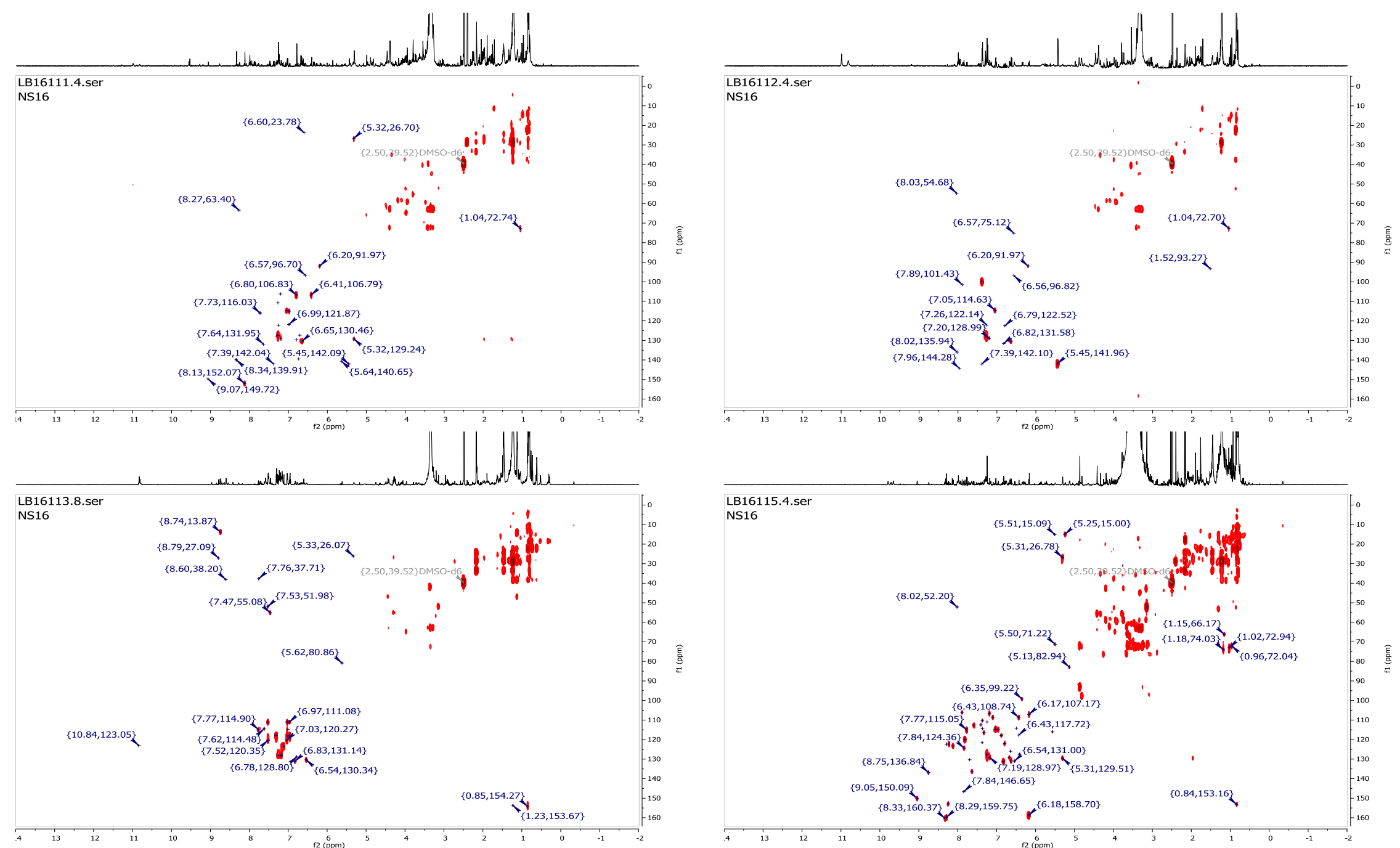

Figure IIA. HSQC-TOCSY NMR spectra of actinomycete crude extracts (continued) (DMSO- $d_{6}$; $800 \mathrm{MHz}$; USC-isolate number given in the top left corner of spectra) 

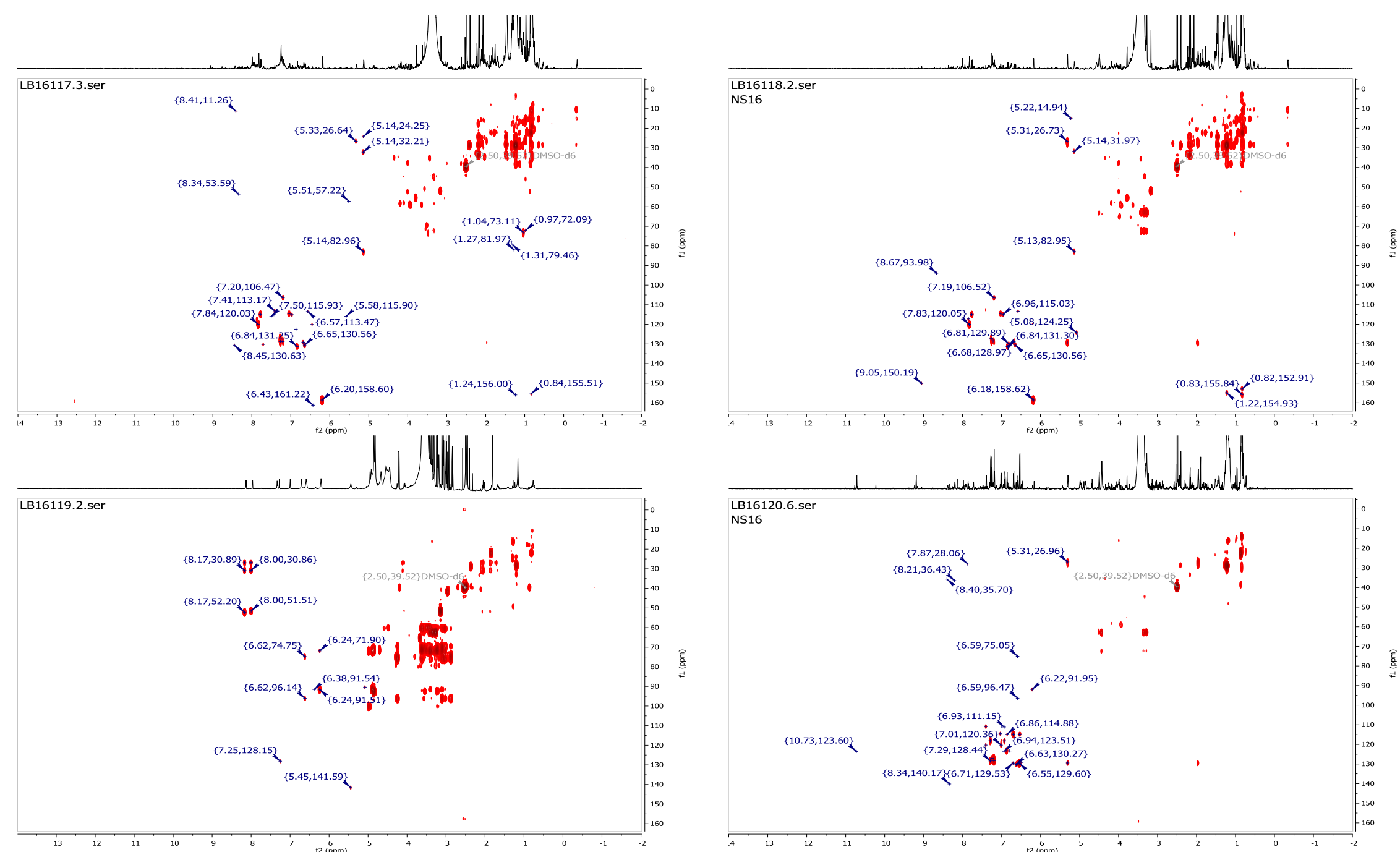

Figure IIA. HSQC-TOCSY NMR spectra of actinomycete crude extracts (continued) (DMSO- $d_{6}$; $800 \mathrm{MHz}$; USC-isolate number given in the top left corner of spectra) 


\section{APPENDIX III - Appendix for Chapter 4}

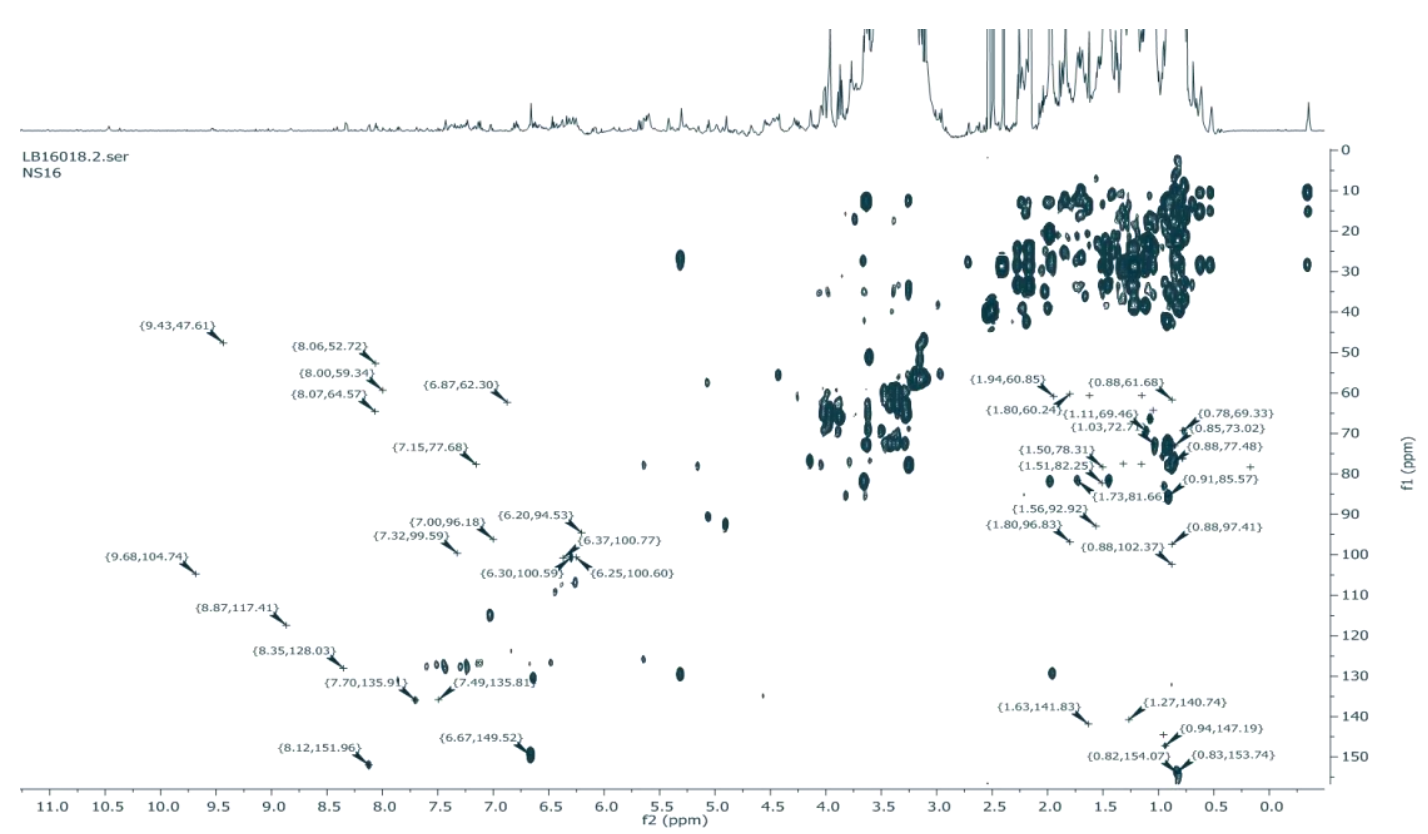

Figure IIIA. HSQC-TOCSY spectrum of USC-16018 EtOAc crude extract (DMSO-d 6 ; 800 MHz)
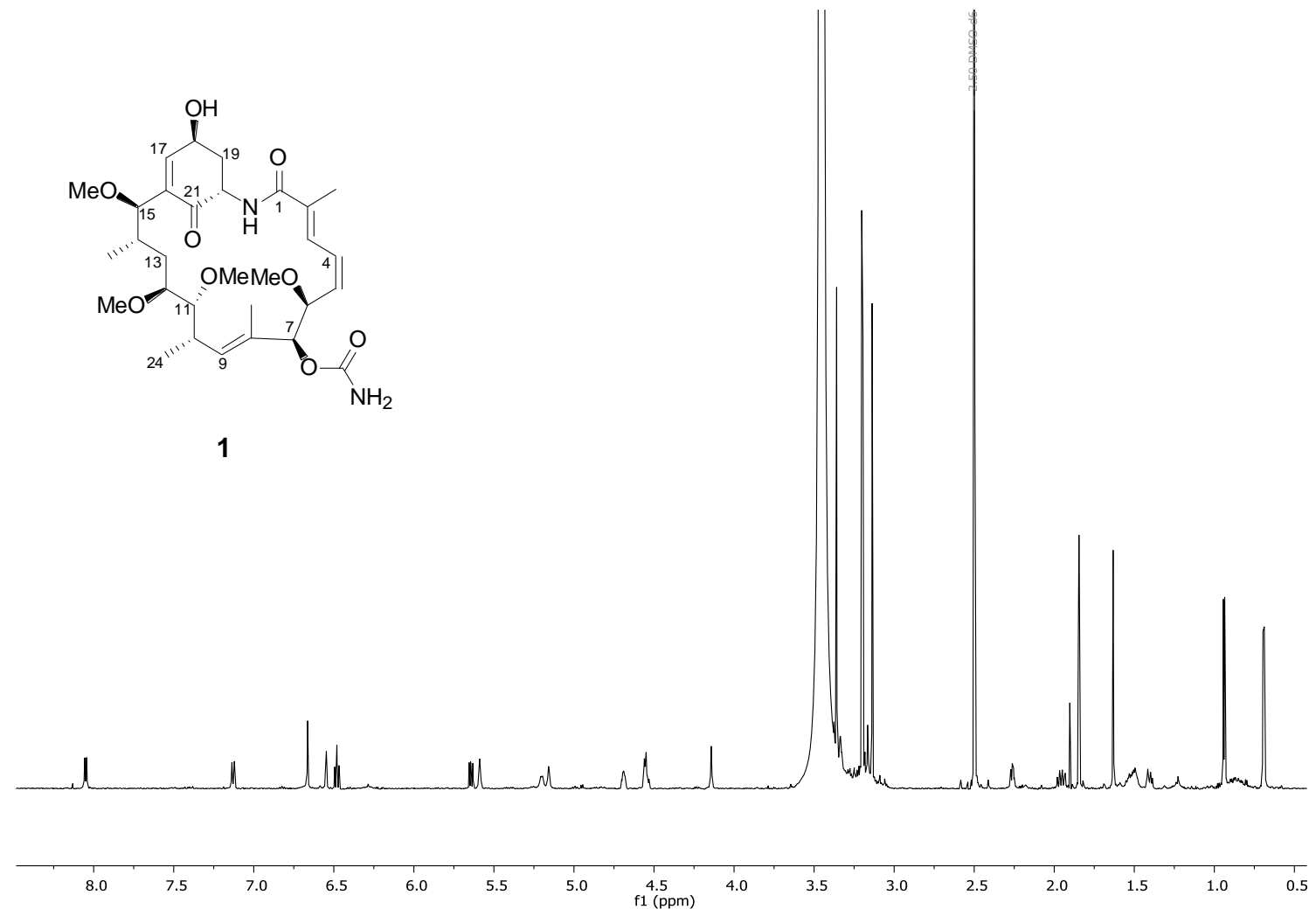

Figure IIIB. ${ }^{1} \mathrm{H}$ NMR spectrum of herbimycin G (1) (DMSO-d 6 ; 800MHz) 


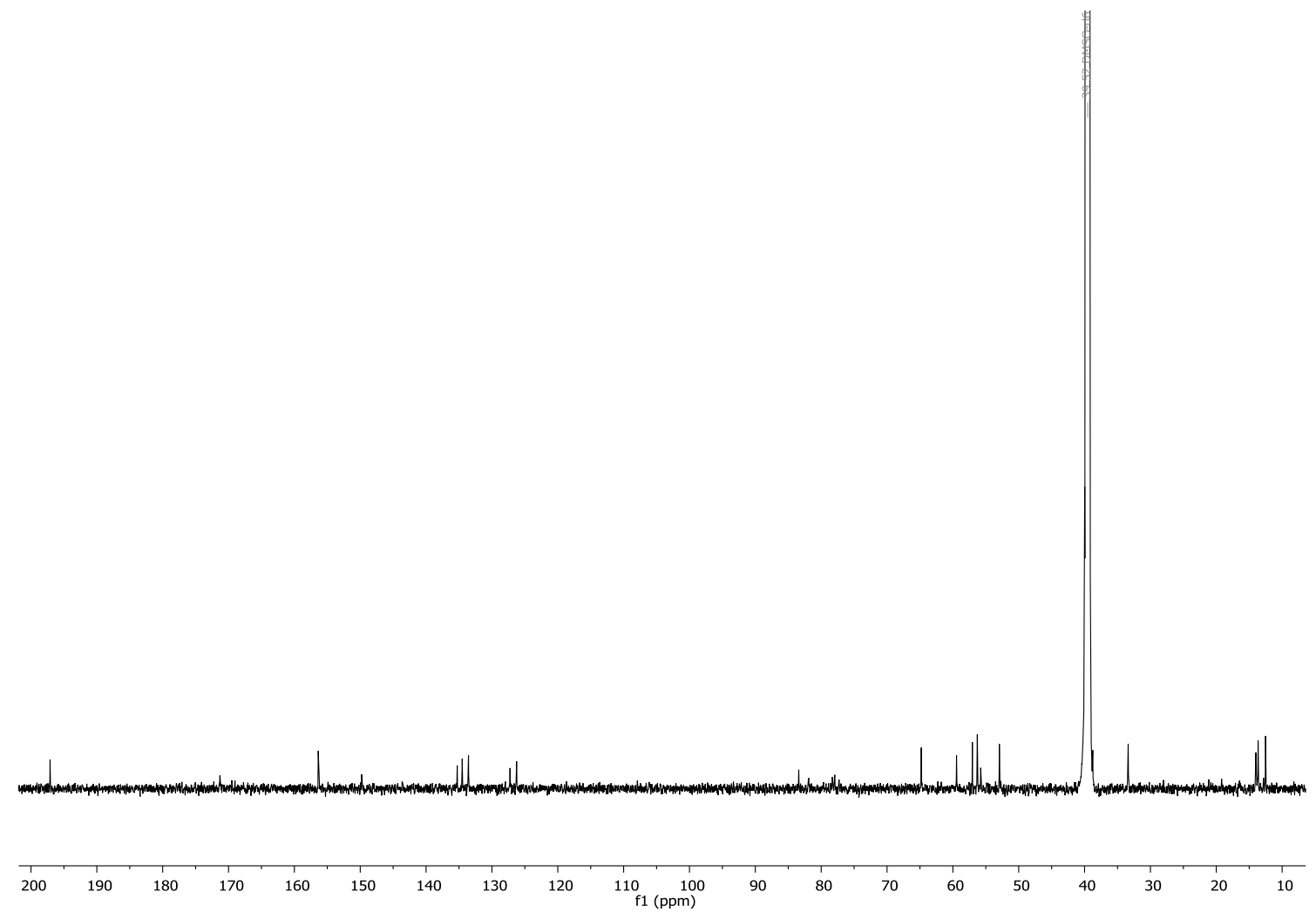

Figure IIIC. ${ }^{13} \mathrm{C}$ NMR spectrum of herbimycin G (1) (DMSO- $d_{6 ;}$ 800MHz)

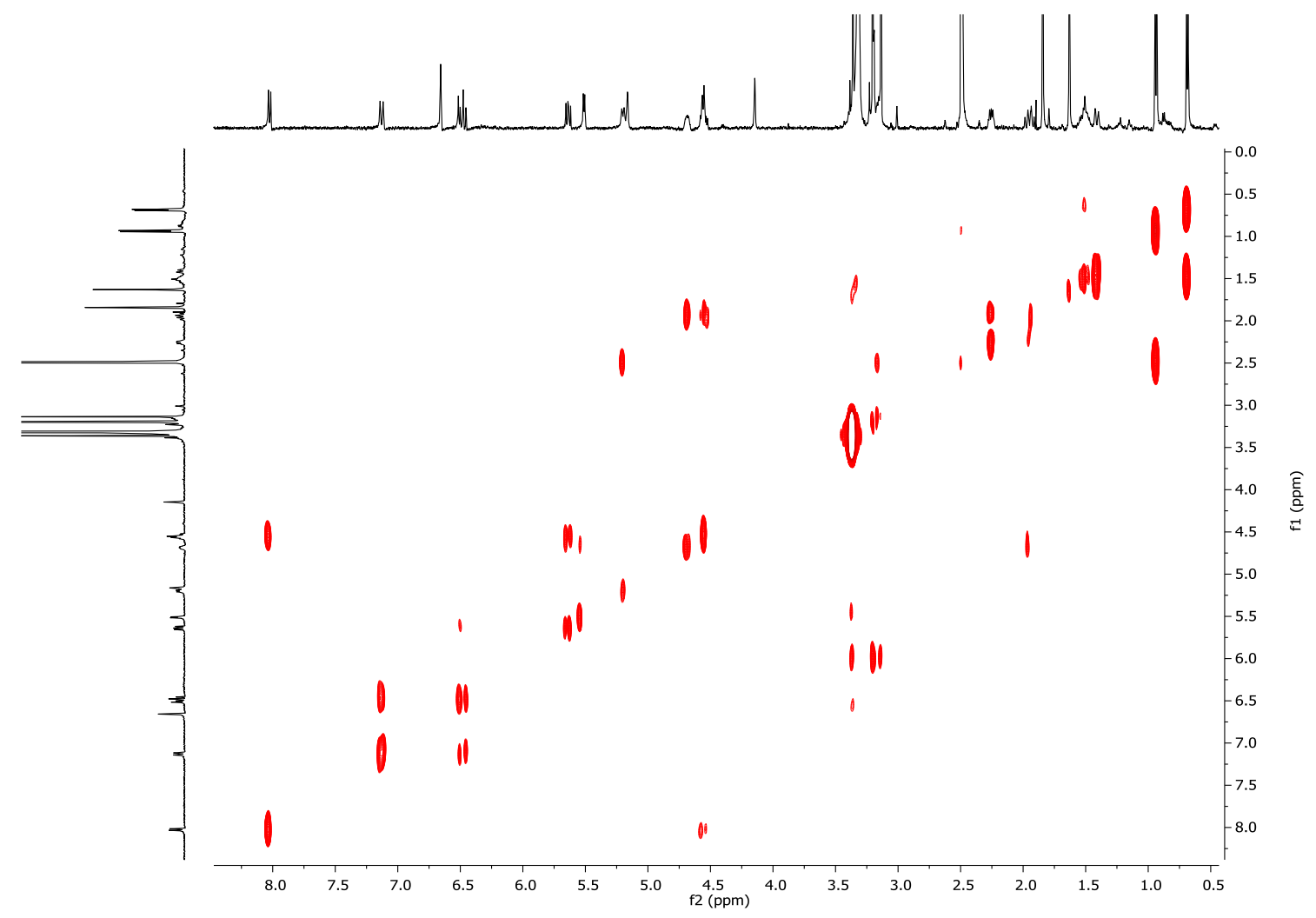

Figure IIID. COSY NMR spectrum of herbimycin G (1) (DMSO- $\left.d_{6} ; 500 \mathrm{MHz}\right)$ 


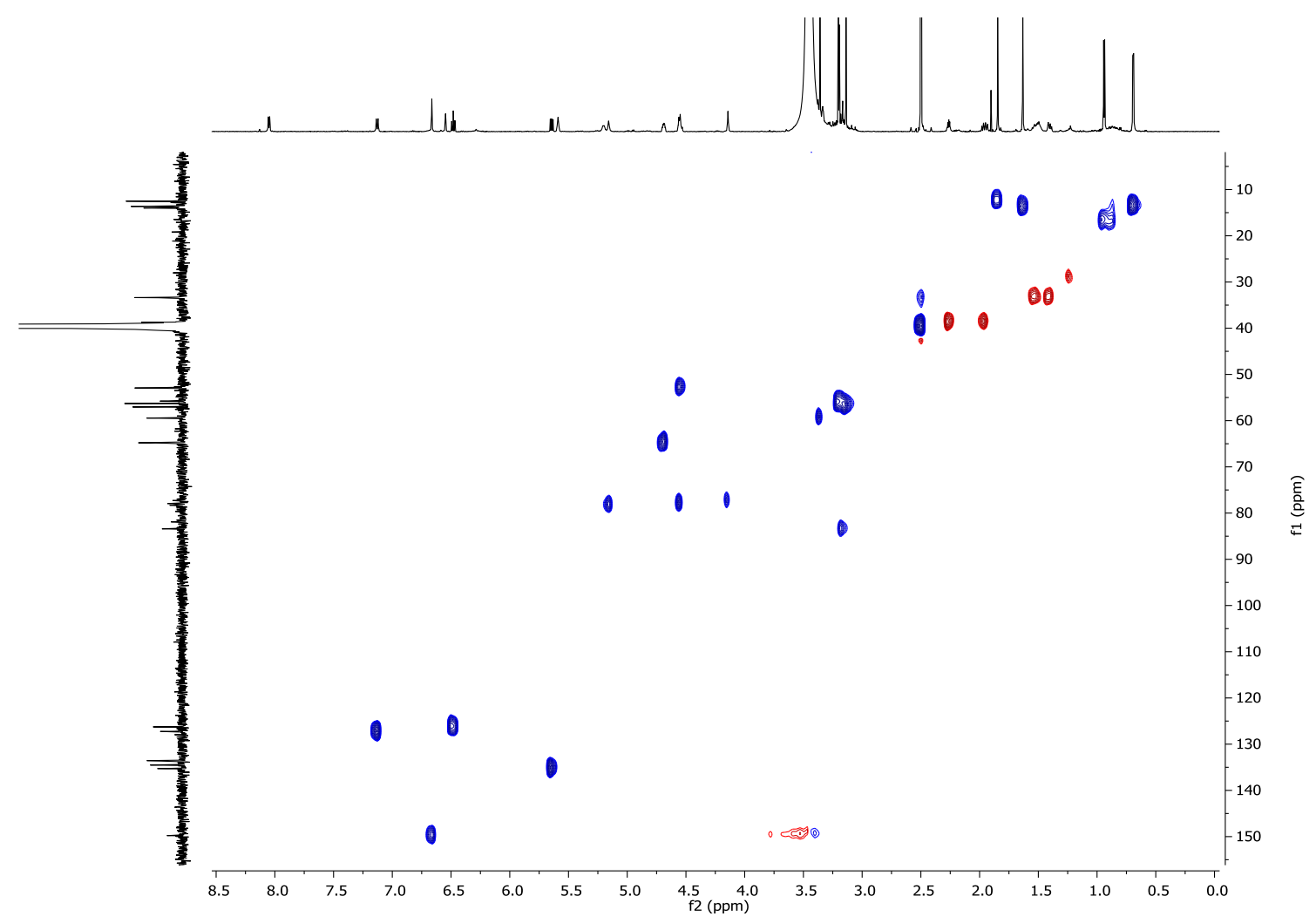

Figure IIIE. HSQC NMR spectrum of herbimycin G (1) (DMSO- $d_{6 ;}$ 800MHz)

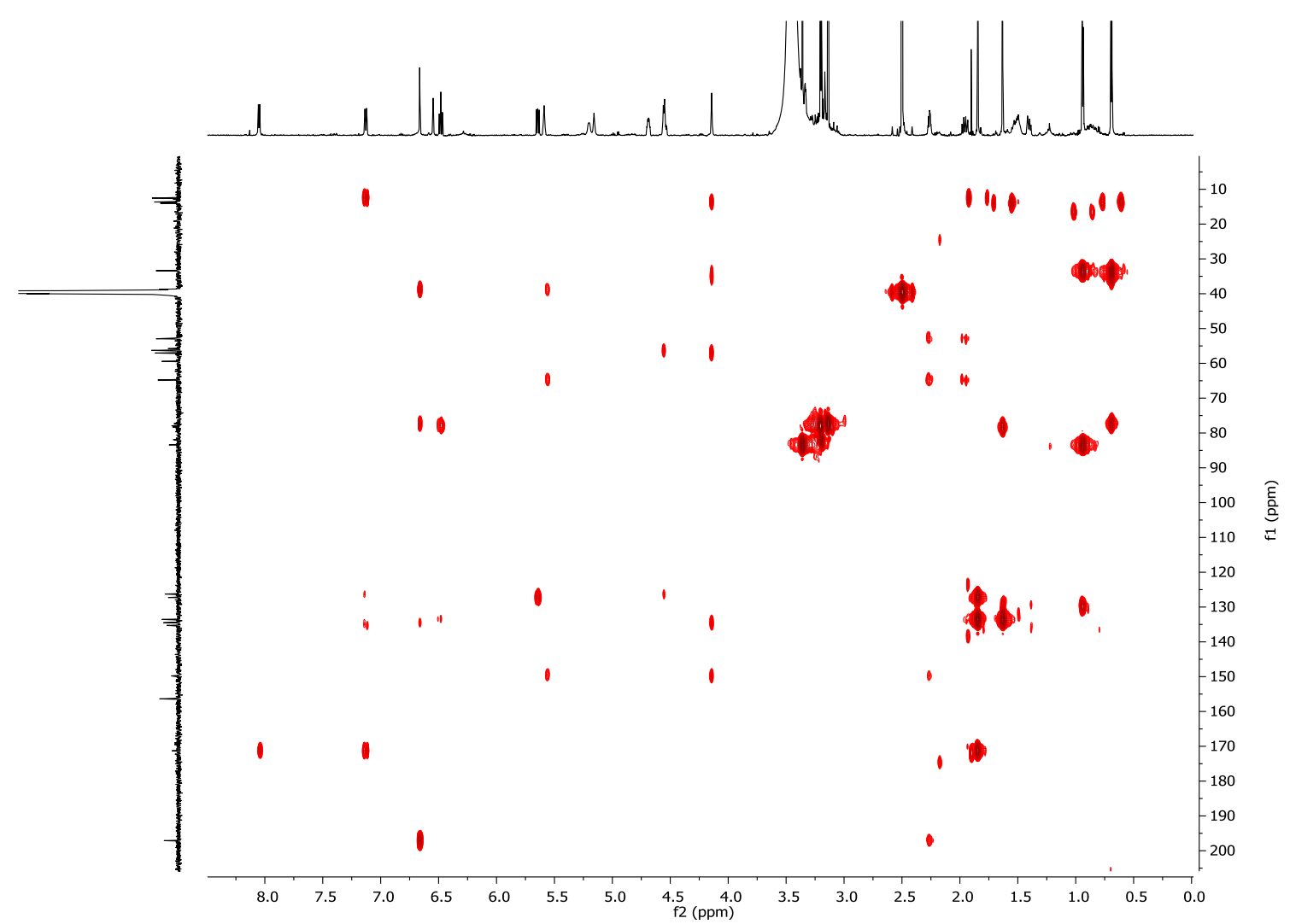

Figure IIIF. HMBC NMR spectrum of herbimycin G (1) (DMSO- $\left.d_{6} ; 800 M H z\right)$ 


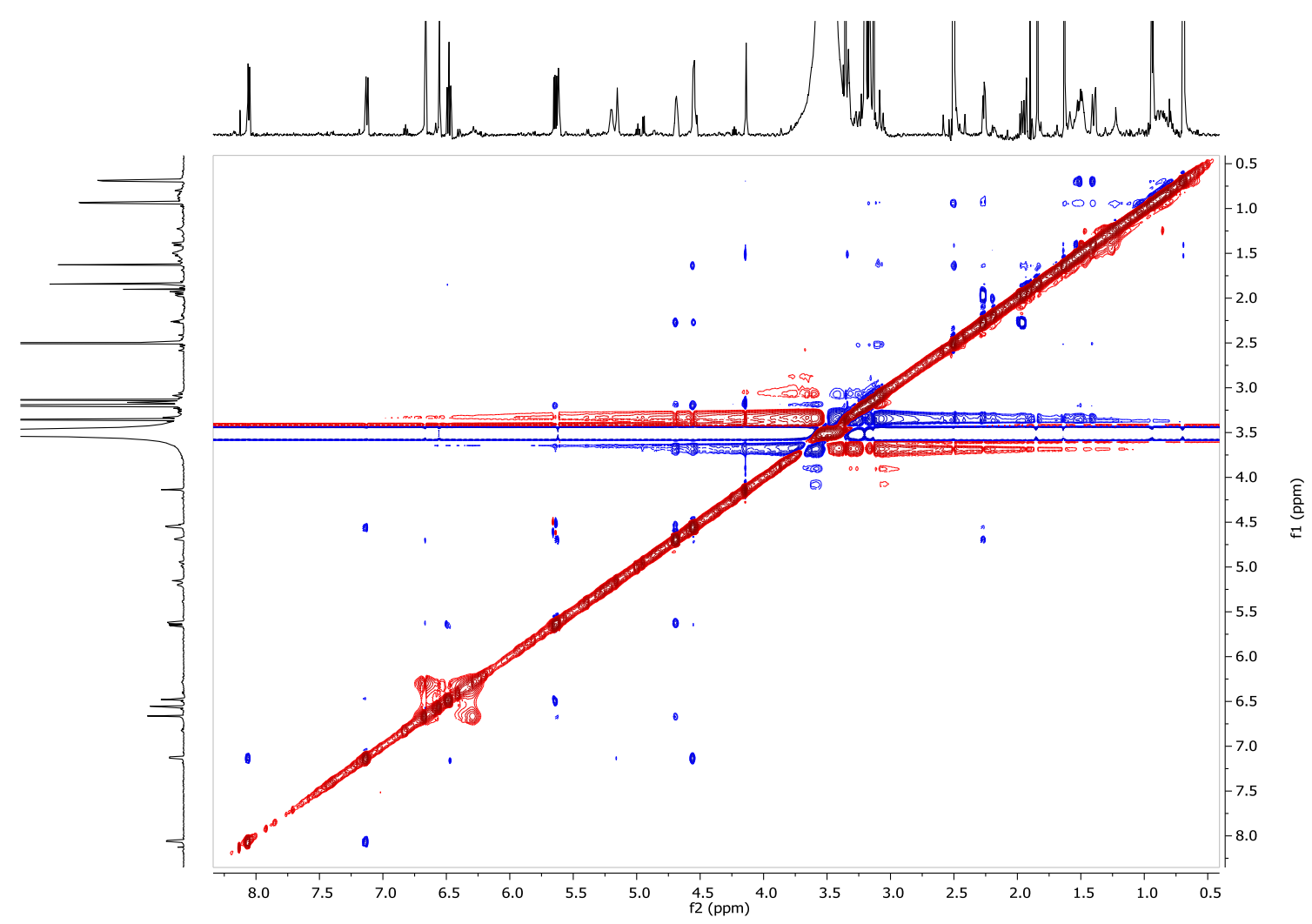

Figure IIIG. ROESY NMR spectrum of herbimycin G (1) (DMSO- $d_{6 ;}$ 800MHz)

Table IIIA. Physiochemical parameters of herbimycins and related ansamysin compounds

\begin{tabular}{lcccccc}
\hline \multicolumn{1}{c}{ Compound } & MW & clogP & HBA & HBD & RB & TPSA \\
\hline HerbA & 574.289 & 2.1592 & 8 & 2 & 2 & 152.48 \\
HerbB & 530.2628 & 2.156 & 7 & 3 & 4 & 154.25 \\
HerbC & 560.2734 & 1.396 & 8 & 3 & 5 & 163.48 \\
HerbD & 647.2877 & 2.505 & 8 & 4 & 6 & 165.67 \\
HerbE & 590.2839 & 2.107 & 9 & 3 & 6 & 172.71 \\
HerbF & 544.2785 & 3.297 & 7 & 3 & 5 & 138.57 \\
HerbG & 578.3203 & 0.6762 & 8 & 3 & 6 & 155.64 \\
HerbA-2 & 666.264 & 1.977 & 8 & 2 & 8 & 152.48 \\
HerbB-2 & 548.2734 & 0.985 & 8 & 4 & 4 & 174.48 \\
HerbB-3 & 546.3577 & 1.011 & 8 & 4 & 4 & 174.48 \\
TAN420A & 548.2734 & 1.178 & 8 & 6 & 4 & 180.8 \\
TAN420B & 546.2577 & 0.634 & 8 & 4 & 4 & 174.48 \\
TAN420E & 576.3047 & 2.703 & 8 & 4 & 6 & 158.8 \\
HeronA & 651.319 & 1.544 & 8 & 4 & 6 & 167.67 \\
Geld & 560.2734 & 2.0573 & 8 & 3 & 5 & 163.48 \\
17-AAG & 585.305 & 3.019 & 8 & 4 & 7 & 166.28 \\
Reblast & 546.2941 & 3.305 & 7 & 4 & 5 & 149.57 \\
MacI & 514.2679 & 2.962 & 6 & 3 & 3 & 145.02 \\
\hline
\end{tabular}




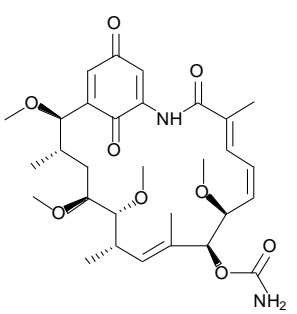

Herbimycin A (HerbA) Chemical Formula: $\mathrm{C} 30 \mathrm{H} 42 \mathrm{~N} 2 \mathrm{O}$ Exact Mass: 574.2890

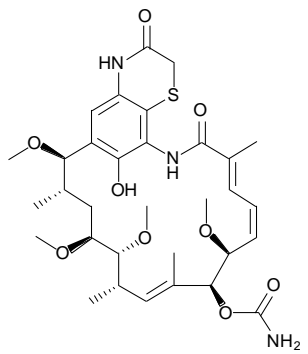

Herbimycin D (HerbD) Chemical Formula: C32H45N3O9S Exact Mass: 647.2877

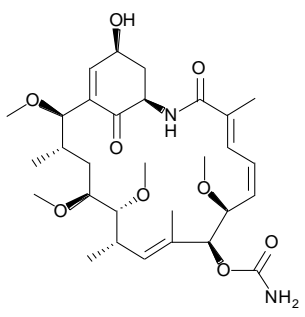

Herbimycin G (HerbG)

Chemical Formula: $\mathrm{C} 30 \mathrm{H} 46 \mathrm{~N} 2 \mathrm{O} 9$ Exact Mass: 578.3203

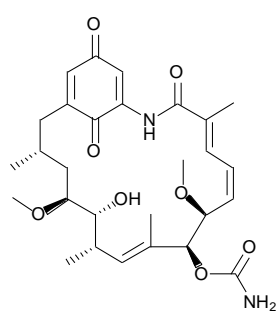

Herbimycin B (HerbB) Chemical Formula: C28H38N2O8 Exact Mass: 530.2628

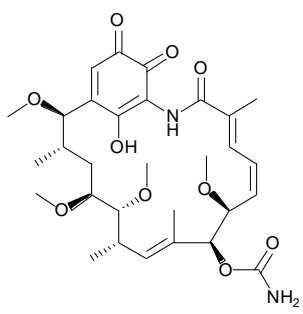

Herbimycin E (HerbE) Chemical Formula: $\mathrm{C} 30 \mathrm{H} 42 \mathrm{~N} 2 \mathrm{O} 10$ Exact Mass: 590.2839

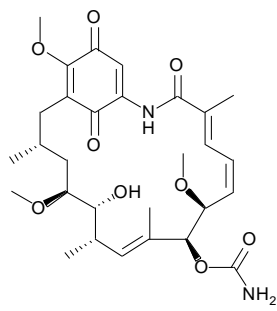

Geldanamycin (Geld)

Chemical Formula: C29H40N2O9 Exact Mass: 560.2734

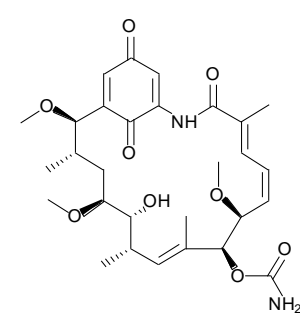

Herbimycin C (HerbC)

Chemical Formula: $\mathrm{C} 29 \mathrm{H} 40 \mathrm{~N} 2 \mathrm{O} 9$ Exact Mass: 560.2734<smiles>COc1c(NC(=O)/C(C)=C/C=C\[C@@H](OC)[C@H](OC(N)=O)/C(C)=C/[C@H](C)C[C@@H](OC)[C@@H](C)OC)cc(O)cc1[C@@H](OC)[C@@H](C)C[C@@H](C)OC</smiles>

Herbimycin $\mathrm{F}$ (HerbF) Chemical Formula: $\mathrm{C} 29 \mathrm{H} 40 \mathrm{~N} 2 \mathrm{O}$ Exact Mass: 544.2785

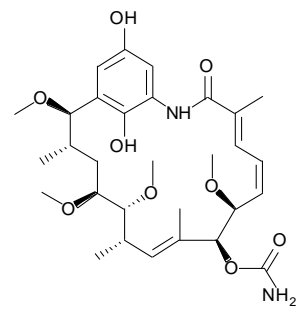

Dihydro-Herbimycin A (TAN420E) Chemical Formula: $\mathrm{C} 30 \mathrm{H} 44 \mathrm{~N} 2 \mathrm{O} 9$
Exact Mass: 576.3047

Figure IIIH. Herbimycin analogues and related ansamycines 


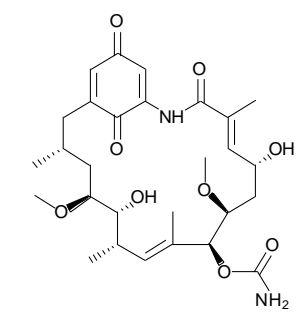

4,5-dihydro-(4S)-4-hydroxyherbimycin $\mathrm{B}$ (HerbB-2)

Chemical Formula: $\mathrm{C} 28 \mathrm{H} 40 \mathrm{~N} 2 \mathrm{O} 9$ Exact Mass: 548.2734

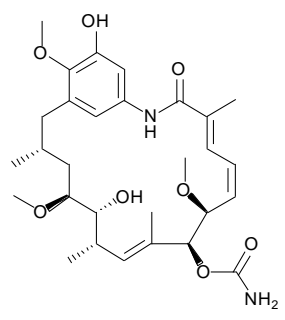

Reblastatin (Reblast) Chemical Formula: $\mathrm{C} 29 \mathrm{H} 42 \mathrm{~N} 2 \mathrm{O} 8$ Exact Mass: 5462941

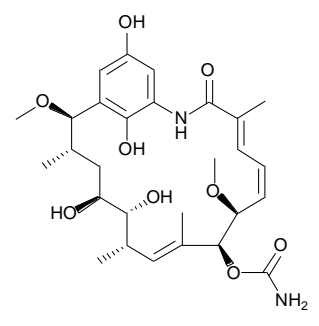

TAN420A
Chemical Formula: $\mathrm{C} 28 \mathrm{H} 40 \mathrm{~N} 2 \mathrm{O} 9$
Exact Mass: 548.2734

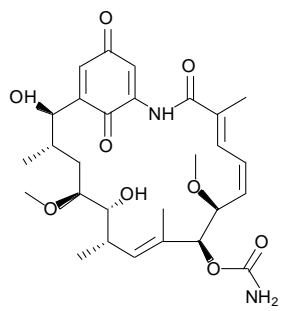

(15S)-15-hydroxyherbimycin B (HerbB-3)

Chemical Formula: C28H38N2O9 Exact Mass: 546.2577

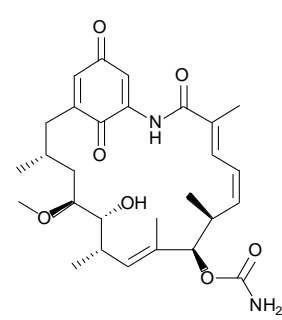

Macbecin I (Macl) Chemical Formula: C28H38N2O7 Exact Mass: 5142679

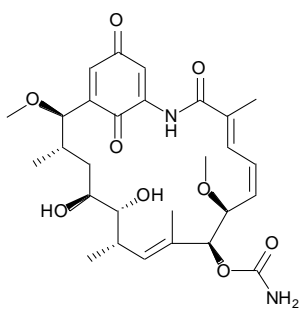

TAN420B
Chemical Formula: $\mathrm{C} 28 \mathrm{H} 38 \mathrm{~N} 2 \mathrm{O} 9$
Exact Mass: 546.2577

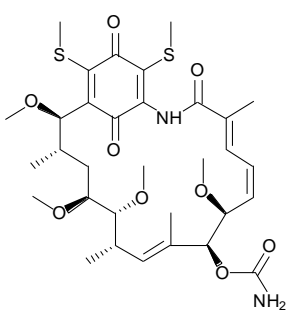

17,19-Dimethylthioherbimycin A (HerbA-2)

Chemical Formula: C32H46N2O9S2 Exact Mass: 666.2645

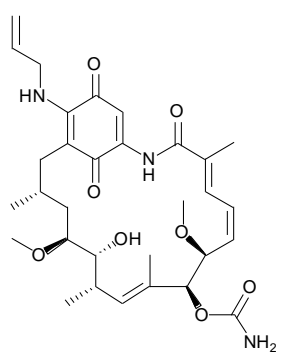

Tanespimycin (17-AAG)

Chemical Formula: $\mathrm{C} 31 \mathrm{H} 43 \mathrm{~N} 3 \mathrm{O} 8$ Exact Mass: 585.3050

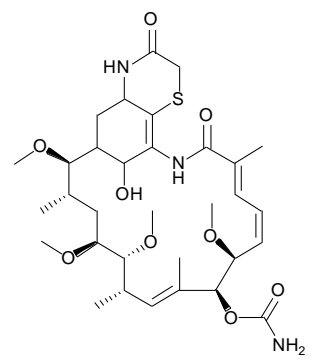

Heronmycin A (HeronA) Chemical Formula: C32H49N3O9S

Figure IIIH. Herbimycin analogues and related ansamycines (cont.) 


\section{APPENDIX IV for Chapter 6}

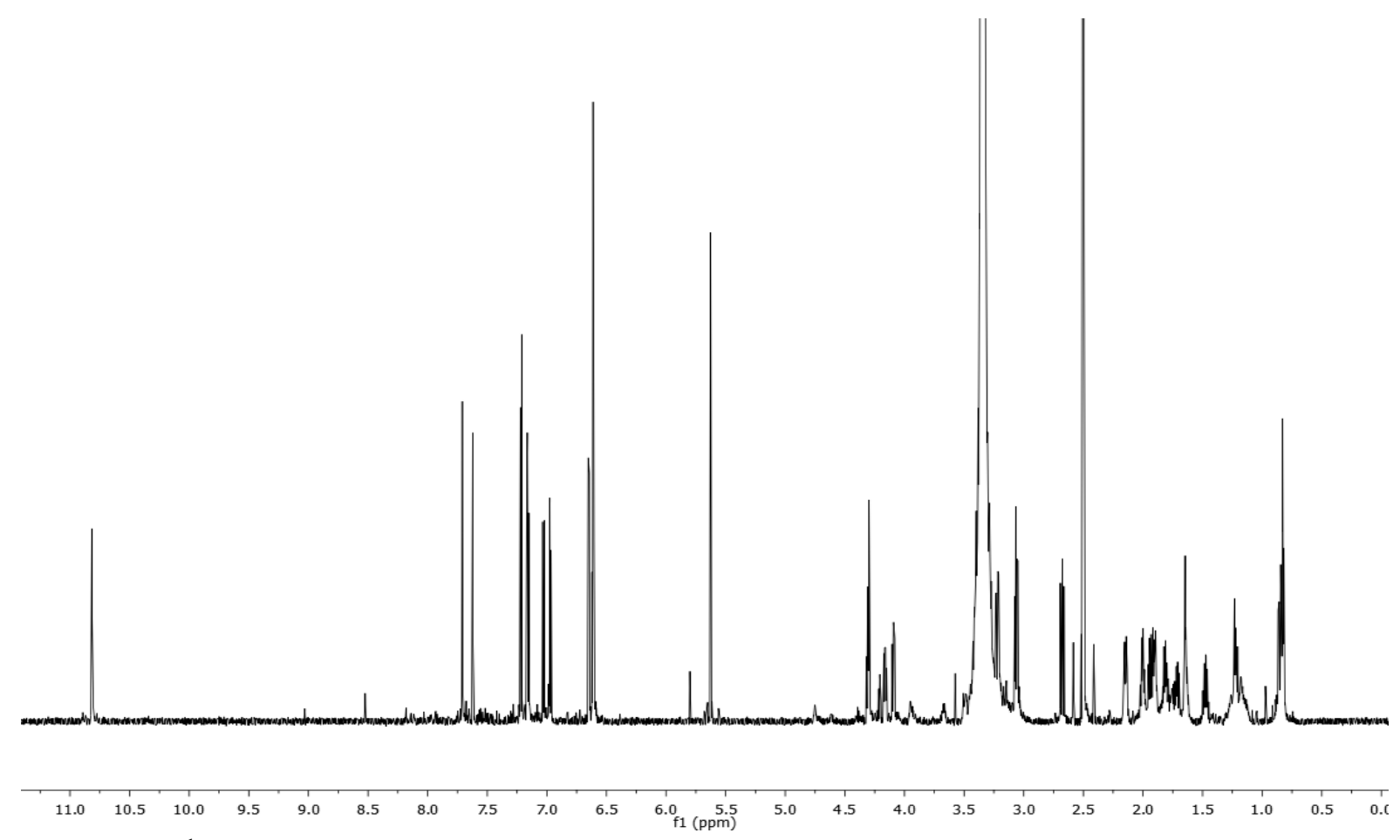

Figure IVA. ${ }^{1} \mathrm{H}$ spectrum of naseseazine C (5) in DMSO- $d_{6}$

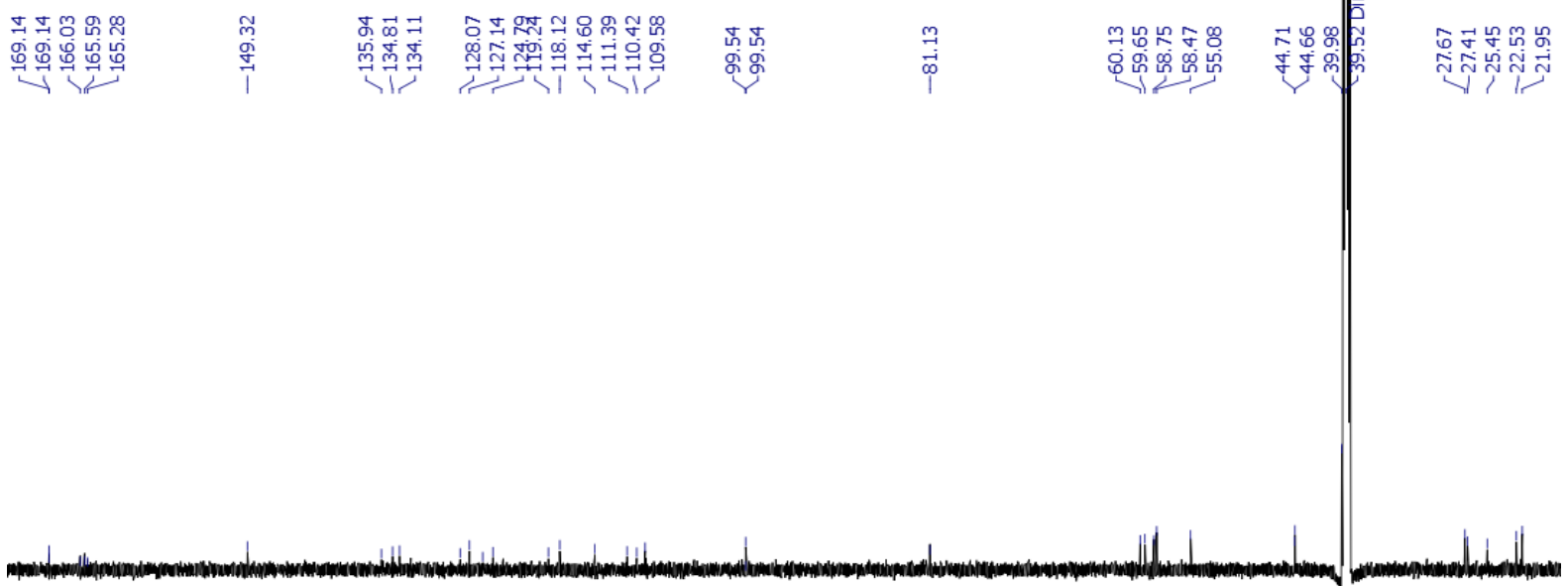

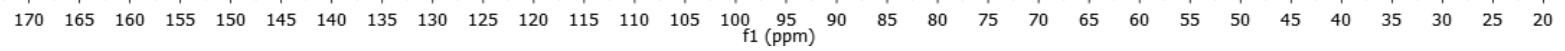

Figure IVB. ${ }^{13} \mathrm{C}$ NMR spectrum of naseseazine C (5) in DMSO- $d_{6}$ 


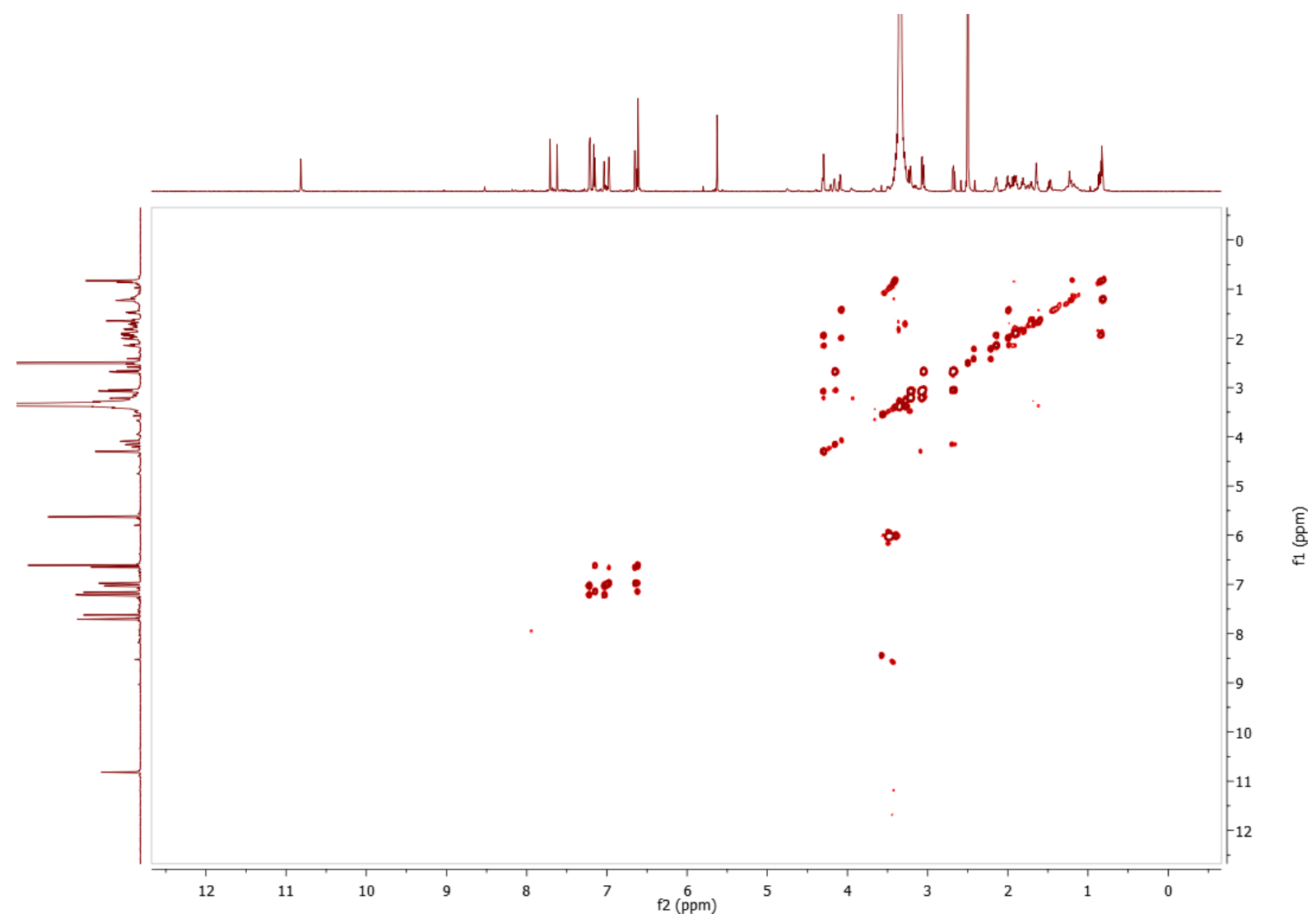

Figure IVC. COSY spectrum of naseseazine C (5) in DMSO-d ${ }_{6}$

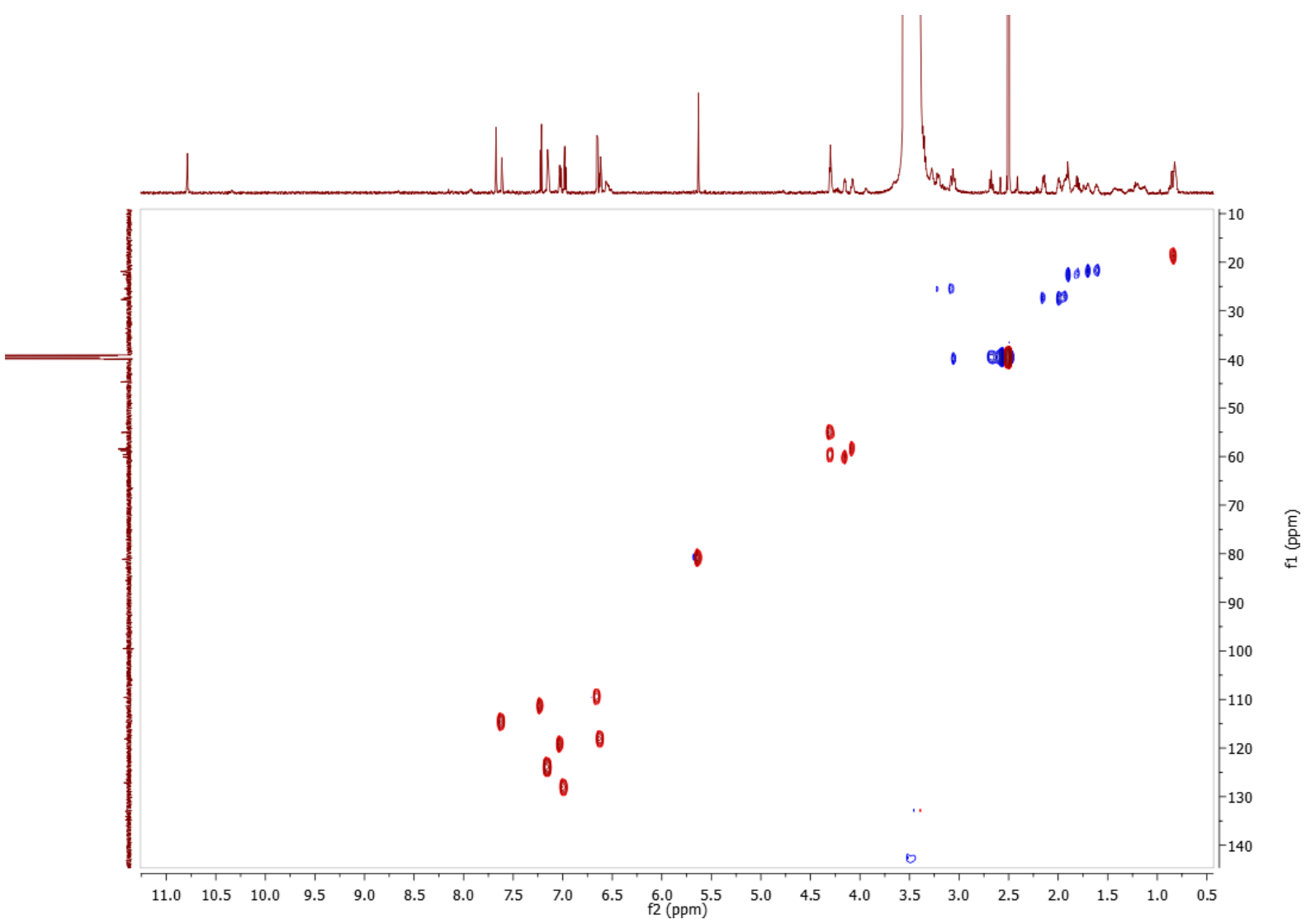

Figure IVD. HSQC spectrum of naseseazine C (5) in DMSO-d d $_{6}$ 


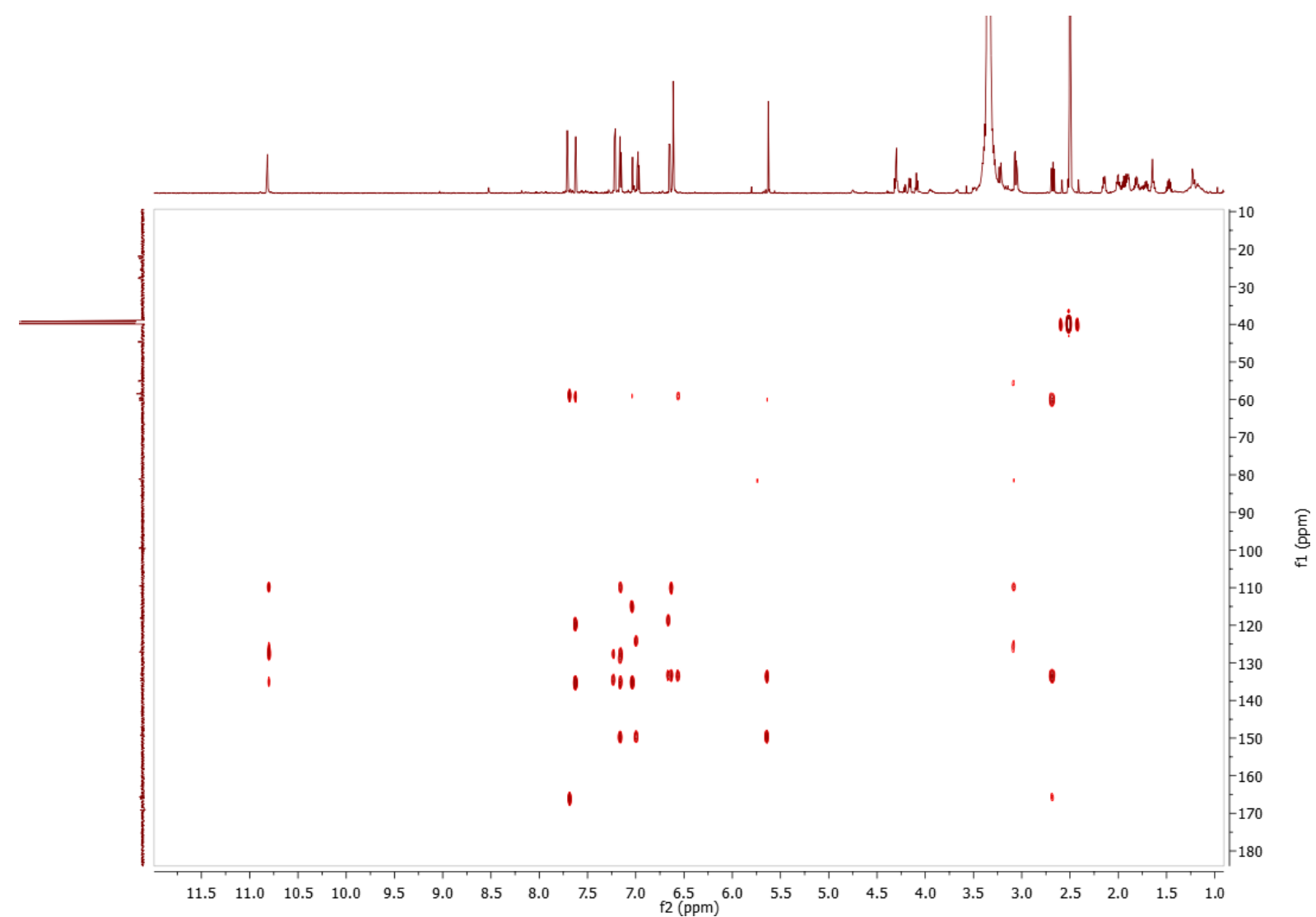

Figure IVE. HMBC spectrum of naseseazine C (5) in DMSO- $d_{6}$

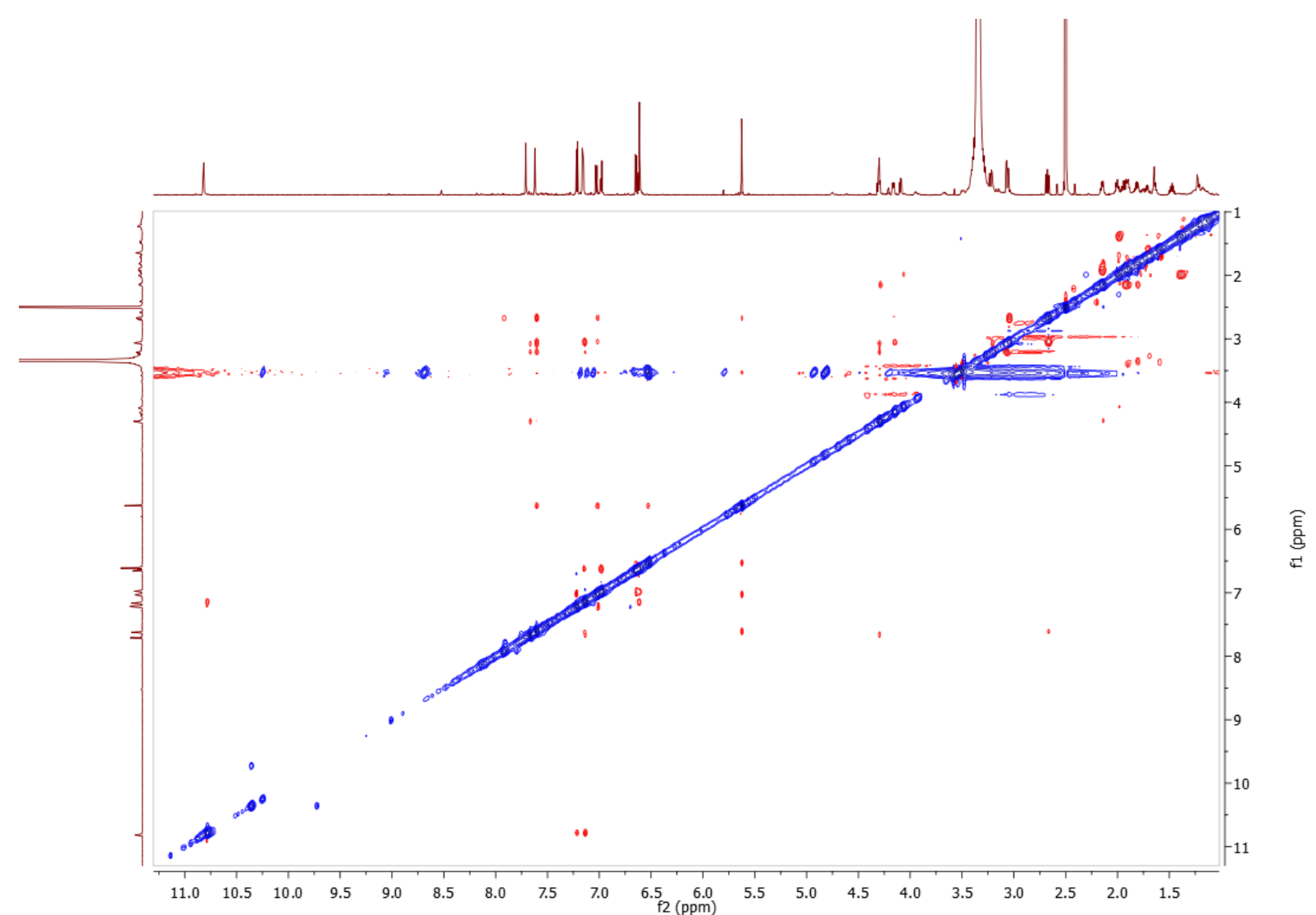

Figure IVF. ROESY spectrum of naseseazine C (5) in DMSO- $d_{6}$ 


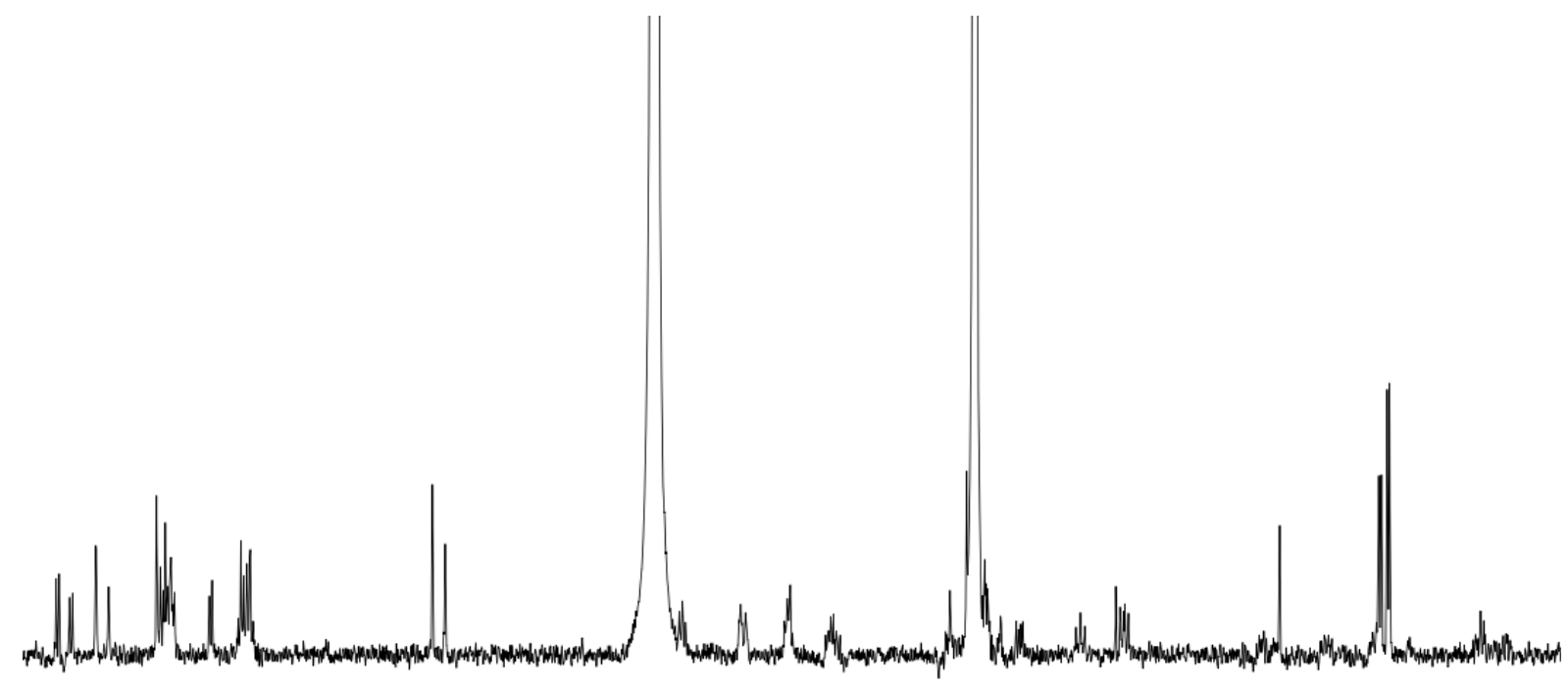

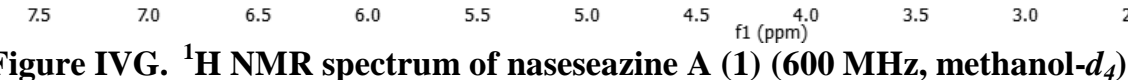

Table IVA. NMR data for naseseazine A (600 MHz, methanol- $\left.d_{4}\right)$

\begin{tabular}{|c|c|c|}
\hline Position & $\delta_{\mathrm{H}}$, multiplet, $(J$ in $\mathrm{Hz})$ & Raju et al. ${ }^{1} \mathrm{H}$ NMR \\
\hline 2 & $5.83, \mathrm{~s}$ & $5.83, \mathrm{~s}$ \\
\hline \multicolumn{3}{|l|}{3} \\
\hline \multicolumn{3}{|l|}{4} \\
\hline 5 & $6.86, \mathrm{~d},(7.5)$ & $6.85, \mathrm{~d},(7.4)$ \\
\hline 6 & 6.67 & $6.67, \mathrm{t},(7.5)$ \\
\hline 7 & $7.05, \mathrm{t}$ & $7.05, t,(7.2)$ \\
\hline 8 & $6.69, \mathrm{~d}(8.0)$ & $6.69, \mathrm{~d},(7.6)$ \\
\hline \multirow{2}{*}{\multicolumn{3}{|c|}{$\begin{array}{c}9 \\
10\end{array}$}} \\
\hline & & \\
\hline 11 & $4.47, \mathrm{dd},(7.5,8.7)$ & $4.64, \mathrm{dd},(8.4,7.4)$ \\
\hline $12 \mathrm{a}$ & $3.36, \mathrm{~m}$ & $3.26, \mathrm{~m}$ \\
\hline $12 \mathrm{~b}$ & $2.61, \mathrm{dd},(9.6,13.8)$ & $3.26, \mathrm{~m}$ \\
\hline \multicolumn{3}{|c|}{ (2) } \\
\hline \multicolumn{3}{|l|}{14} \\
\hline \multirow{2}{*}{\multicolumn{3}{|c|}{$4.1 J, \mathrm{q},(0.9)$}} \\
\hline & & \\
\hline 17 & $1.38, \mathrm{~d},(6.7)$ & $1.38, \mathrm{~d},(6.9)$ \\
\hline \multicolumn{3}{|l|}{18} \\
\hline $2^{\prime}$ & $7.11, \mathrm{~s}$ & $7.11, \mathrm{~s}$ \\
\hline \multicolumn{3}{|l|}{$3^{\prime}$} \\
\hline \multicolumn{3}{|l|}{$4^{\prime}$} \\
\hline $5^{\prime}$ & $7.57, \mathrm{~d},(8.7)$ & $7.57, \mathrm{~d},(8.4)$ \\
\hline $6^{\prime}$ & $7.04, d,(8.7)$ & $7.02, d,(8.4)$ \\
\hline \multicolumn{3}{|c|}{$x^{2}$} \\
\hline $8^{\prime}$ & $7.40, d,(1.8)$ & $7.40, \mathrm{~s}$ \\
\hline \multicolumn{3}{|c|}{$1.10, \mathrm{a},(1.0)$} \\
\hline \multicolumn{3}{|l|}{$10^{\prime}$} \\
\hline $11^{\prime}$ & 4.40 , broad $t,(4.4)$ & 4.39, broad $t,(4.5)$ \\
\hline $12^{\prime} \mathrm{a}$ & 3.28 & $3.28, \mathrm{~m}$ \\
\hline $12 \mathrm{~b}$ & 3.3 & $3.30, \mathrm{~m}$ \\
\hline \multicolumn{3}{|l|}{$13^{\prime}$} \\
\hline \multicolumn{3}{|l|}{$14^{\prime}$} \\
\hline $15^{\prime}$ & 3.97 & $3.97, \mathrm{dd},(10.8,6.6)$ \\
\hline \multicolumn{3}{|r|}{ 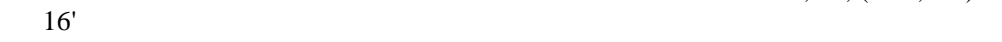 } \\
\hline 17 'a & $0.95, \mathrm{~m}$ & $0.93, \mathrm{~m}$ \\
\hline $17^{\prime} \mathrm{b}$ & $1.96, \mathrm{~m}$ & $1.97, \mathrm{~m}$ \\
\hline $18^{\prime} \mathrm{a}$ & $1.42, \mathrm{~m}$ & $1.43, \mathrm{~m}$ \\
\hline $18^{\prime} \mathrm{b}$ & $1.66, \mathrm{~m}$ & $1.66, \mathrm{~m}$ \\
\hline 19 'a & $3.26, \mathrm{~m}$ & $3.24, \mathrm{~m}$ \\
\hline $19^{\prime} \mathrm{b}$ & $3.42, \mathrm{~m}$ & $3.42, \mathrm{dt},(11.8,8.1)$ \\
\hline
\end{tabular}




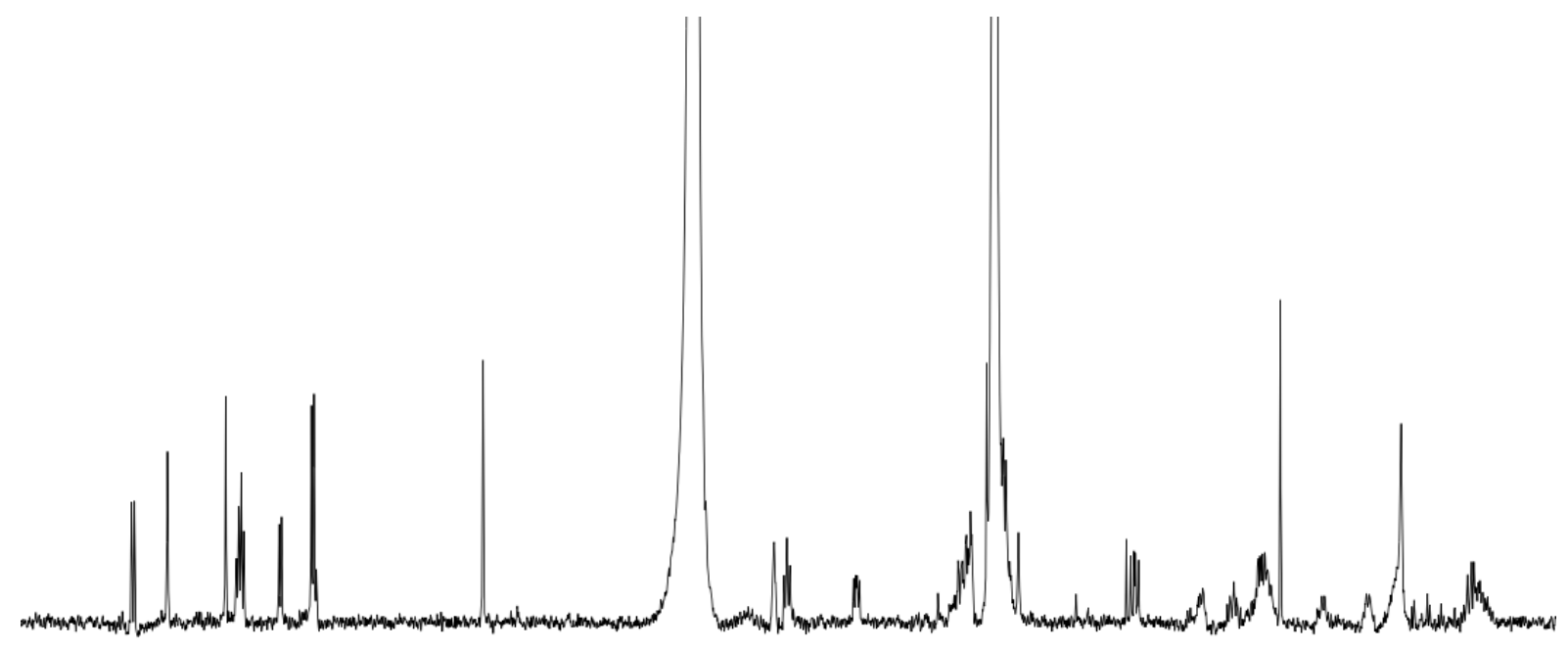

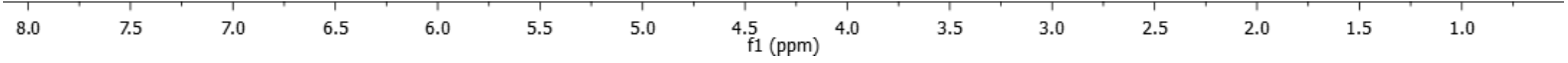

Figure IVH. ${ }^{1} \mathrm{H}$ NMR spectrum of naseseazine B (2) $\left(600 \mathrm{MHz}\right.$, methanol- $\left.d_{4}\right)$

Table IVB. NMR data for naseseazine B (600 MHz, methanol- $\left.d_{4}\right)$

\begin{tabular}{|c|c|c|}
\hline Position & $\delta_{\mathrm{H}}$, multiplet, $(\mathrm{J}$ in $\mathrm{Hz})$ & Raju et al. ${ }^{1}$ H NMR \\
\hline 2 & $5.83, \mathrm{~s}$ & $5.85, \mathrm{~s}$ \\
\hline \multicolumn{3}{|l|}{3} \\
\hline \multicolumn{3}{|l|}{4} \\
\hline 5 & $6.85, \mathrm{dd},(8.1,1.2)$ & $6.84, \mathrm{dt},(7.2,0.9)$ \\
\hline 6 & $6.66, \mathrm{td},(7.7,1.1)$ & $6.68, \mathrm{t},(7.6)$ \\
\hline 7 & $7.04, \mathrm{td},(7.7,1.1)$ & $7.06, \mathrm{td},(7.6,1.3)$ \\
\hline 8 & $6.67, \mathrm{~d},(7.7)$ & $6.69, \mathrm{~d},(7.6)$ \\
\hline \multicolumn{3}{|l|}{9} \\
\hline \multicolumn{3}{|l|}{10} \\
\hline 11 & 4.74 & $4.75, \mathrm{dd},(10.2,8.7)$ \\
\hline $12 \mathrm{a}$ & $2.59, \mathrm{dd},(14.1,9.9)$ & $2.59, \mathrm{dd},(13.8,10.2)$ \\
\hline $12 b$ & $3.25, \mathrm{~m}$ & $3.27, \mathrm{~m}$ \\
\hline \multicolumn{3}{|l|}{13} \\
\hline \multicolumn{3}{|l|}{14} \\
\hline 15 & $4.32, \mathrm{dd},(7.1,9.7)$ & $4.33, \mathrm{dd},(9.5,7.1)$ \\
\hline \multicolumn{3}{|c|}{ 管, } \\
\hline $17 \mathrm{a}$ & $2.27, \mathrm{~m}$ & $2.11, \mathrm{~m}$ \\
\hline $17 \mathrm{~b}$ & $2.10, \mathrm{~m}$ & $2.28, \mathrm{~m}$ \\
\hline $18 \mathrm{a}$ & 1.97, m & $1.95, \mathrm{~m}$ \\
\hline $18 \mathrm{~b}$ & $2.00, \mathrm{~m}$ & $2.00, \mathrm{~m}$ \\
\hline $19 \mathrm{a}$ & $3.45, \mathrm{~m}$ & $3.44, \mathrm{~m}$ \\
\hline $19 b$ & $3.48, \mathrm{~m}$ & $3.49, \mathrm{~m}$ \\
\hline $2^{\prime}$ & $7.10, \mathrm{~s}$ & $7.12, \mathrm{~s}$ \\
\hline \multicolumn{3}{|l|}{$3^{\prime}$} \\
\hline \multicolumn{3}{|l|}{$4^{\prime}$} \\
\hline $5^{\prime}$ & $7.56, \mathrm{~d},(8.5)$ & $7.58, \mathrm{~d},(8.4)$ \\
\hline $6^{\prime}$ & $7.02, \mathrm{dd},(8.5,1.7)$ & $7.03, \mathrm{dd},(8.4,1.8)$ \\
\hline \multicolumn{3}{|c|}{ 然 } \\
\hline $8^{\prime}$ & $7.39, \mathrm{~d},(1.7)$ & $7.41, \mathrm{~d},(1.4)$ \\
\hline \multicolumn{3}{|l|}{$9^{\prime}$} \\
\hline \multicolumn{3}{|l|}{$10^{\prime}$} \\
\hline $11^{\prime}$ & 4.38 , broad t $(4.6)$ & 4.40 , broad t, (4.7) \\
\hline $12^{\prime} \mathrm{a}$ & $3.27, \mathrm{~m}$ & $3.28, \mathrm{~m}$ \\
\hline $12^{\prime} \mathrm{b}$ & $3.33, \mathrm{~m}$ & $3.32, \mathrm{~m}$ \\
\hline \multicolumn{3}{|l|}{$13^{\prime}$} \\
\hline \multicolumn{3}{|l|}{$14^{\prime}$} \\
\hline $15^{\prime}$ & 3.97, ddd, $(11.4,6.9,1.7)$ & 3.99, ddd, $(11.4,6.6,1.6)$ \\
\hline \multicolumn{3}{|c|}{ (1) } \\
\hline 17 'a & $0.93, \mathrm{~m}$ & $0.92, \mathrm{~m}$ \\
\hline $17 \mathrm{~b}$ & $1.96, \mathrm{~m}$ & $1.97, \mathrm{~m}$ \\
\hline $18^{\prime} \mathrm{a}$ & $1.43, \mathrm{~m}$ & $1.44, \mathrm{~m}$ \\
\hline $18^{\prime} \mathrm{b}$ & $1.66, \mathrm{~m}$ & $1.67, \mathrm{~m}$ \\
\hline $19^{\prime} \mathrm{a}$ & $3.24, \mathrm{~m}$ & $3.24, \mathrm{~m}$ \\
\hline $19 ' b$ & $3.43, \mathrm{~m}$ & $3.43, \mathrm{~m}$ \\
\hline
\end{tabular}


Table IVC. Anti-plasmodial activity of naseseazine A-C and chloroquine

\begin{tabular}{|c|c|c|c|c|c|}
\hline & Expt 1 & Expt 2 & Expt 3 & & \\
\hline $\begin{array}{c}\text { Naseseazine A } \\
\text { Naseseazine B }\end{array}$ & $47 \%$ at $20 \mathrm{uM}$ & $35 \%$ at $20 \mathrm{uM}$ & $28 \%$ at $20 \mathrm{uM}$ & & \\
\hline & $\mathrm{IC}_{50} \mathrm{uM}$ & $\mathrm{IC}_{50} \mathrm{uM}$ & $\mathrm{IC}_{50} \mathrm{uM}$ & ${\text { Average } \mathrm{IC}_{50} \mathrm{uM}}$ & stdev \\
\hline Naseseazine C & 4.87 & 3.06 & 2.62 & 3.52 & 1.19 \\
\hline Chloroquine* & 0.0118 & 0.00495 & 0.00754 & 0.0081 & 0.0035 \\
\hline
\end{tabular}

*positive control 


\section{APPENDIX V - In situ iChip isolation techniques}

The isolation chip (iChip) is an effective version of diffusion chambers that can also be deployed in situ. ${ }^{1}$ The iChip consists of hundreds of miniature diffusion chambers of approximately $1 \mathrm{~mm}$ diameter in a central plate, which is dipped into a microbial suspension in molten agar. ${ }^{1}$ Semi-permeable membranes cover the central plate allowing diffusion of nutrients into the chamber, but restrict the movement of the cells to the outside environment. Two supporting plates are screwed to the central plate providing sufficient pressure to seal the isolation (Figure VA). ${ }^{1}$ This device allows simultaneous isolation of environmental bacteria. Initially, an environmental sample, i.e. sediment, is diluted so that approximately one bacterial cell is delivered to each miniature chamber, the device is then sealed with semi-permeable membranes between central and side plates, and incubated back in the natural environment where the sample was taken from originally. Once a colony of sufficient cells is produced, it is likely that the previously uncultured isolates are able to grow in vitro. This isolation approach has recently led to a major breakthrough in microbial research as the novel bacterium Eleftheria terrae could be isolated from soil samples. ${ }^{2}$ This bacterium is the producer of the antibiotic teixobactin (22), the first antibiotic discovered in the last 30 years without detectable resistance. ${ }^{2}$ To date, no marine bacteria have been isolated with the iChip, this was probably due to the so far limited incubation time of only two weeks. ${ }^{3}$ However, it should be possible to isolate marine bacteria with optimised iChip conditions in the future.

\section{iChip design and assembly}

The software SolidWorks was used to design the iChip assembly. Ten iChips consisting of hydrophobic plastic polyoxymethylene were laser cut by Griffith School of Engineering. The central plate, $40.5 \mathrm{~mm} \times 20.25 \mathrm{~mm} \times 2 \mathrm{~mm}$, is supported by two symmetrical side plates of the same dimensions $40.5 \mathrm{~mm} \times 20.25 \mathrm{~mm}$ x $2 \mathrm{~mm}$. All plates have six rows of fifteen $1 \mathrm{~mm}$ diameter through holes ( $2.25 \mathrm{~mm}$ from centre to centre) eventuating to 90 wells per iChip.

All plates will be sterilised in ethanol, followed by drying in a laminar flow hood and rinsed in particle-free DNA- grade water prior to the sampling event. The assemblies will be transported in sterile plastic bags. A solution of the growth media will be supplemented with $100 \mu \mathrm{g} / \mathrm{mL}$ cycloheximide to prevent fungal growth and injected with diluted sponge extract after autoclaving. Therefore, approximately $1.5 \mu \mathrm{L}$ of the microbial infused media will be dispensed into the iChip wells. Next, semi-permeable polycarbonate membranes of $0.03 \mu \mathrm{m}$ pore 
size are applied on each side of the central plate and fixed tightly by screwing the side plates to the central plate; excess membrane material will be cut off. The assembly will subsequently be incubated in living sponges for a minimum of 4 weeks; thereupon the iChip will be cut out of the sponge tissue, placed in sterile seawater and transported back to the laboratory. In the laboratory, the central plate will be examined under a compound microscope for colony counts. The well contents of the iChips will be streaked onto agar plates to test the ability of the microorganisms to grow outside the iChip and for colony purification.

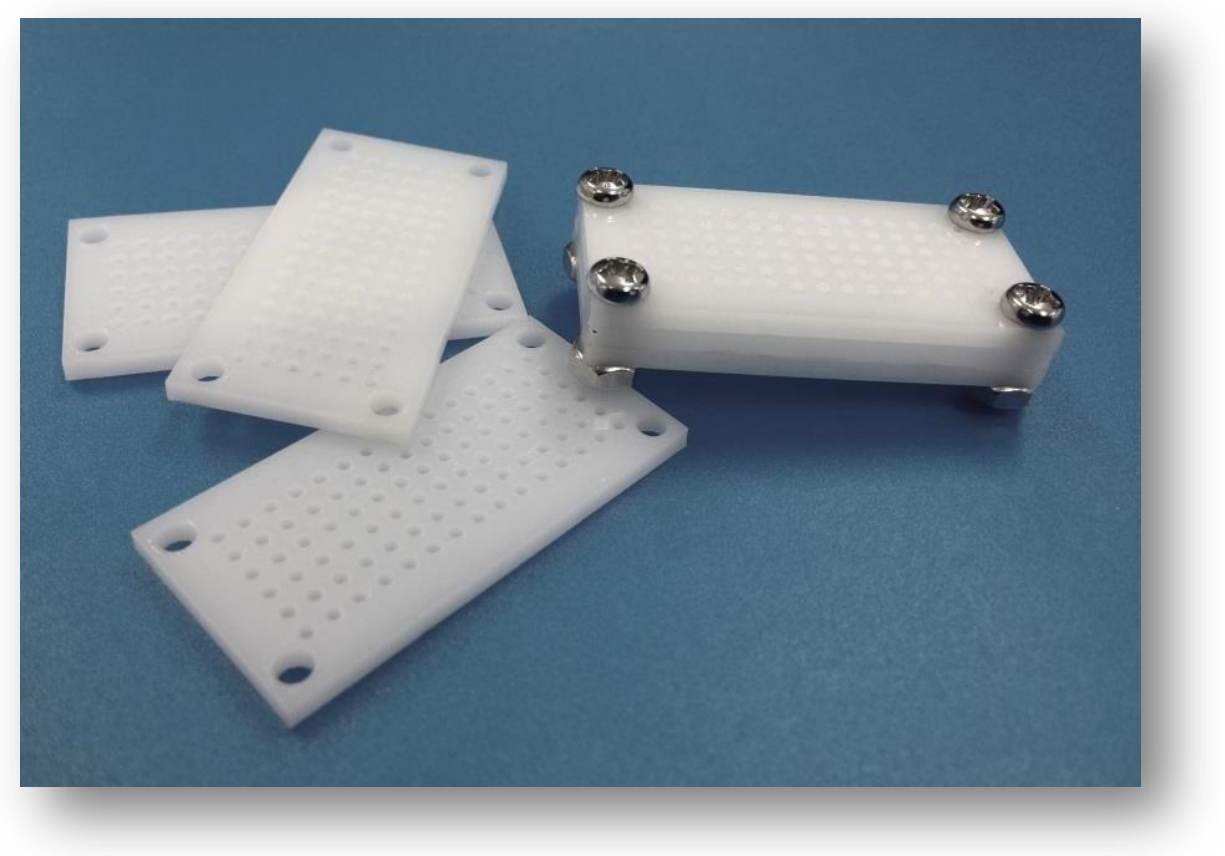

Figure VA. iChip prototype

(1) Lewis, K.; Epstein, S.; D'Onofrio, A.; Ling, L. L. The Journal of antibiotics 2010, 63, 468.

(2) Ling, L. L.; Schneider, T.; Peoples, A. J.; Spoering, A. L.; Engels, I.; Conlon, B. P.; Mueller, A.; Schäberle, T. F.; Hughes, D. E.; Epstein, S. Nature 2015.

(3) Hameş-KocabaŞ, E. E.; Ataç, U. Journal of microbiological methods 2012, 88, 342. 